\title{
Assessment of Unmanned Aerial Systems and lidar for the Utility Vegetation Management of Electrical Distribution Rights-of-Ways
}

\author{
Matthew R. Walker \\ mwalke18@gmail.com
}

Follow this and additional works at: https://researchrepository.wvu.edu/etd

Part of the Forest Management Commons, Power and Energy Commons, and the Risk Analysis

\section{Commons}

\section{Recommended Citation}

Walker, Matthew R., "Assessment of Unmanned Aerial Systems and lidar for the Utility Vegetation Management of Electrical Distribution Rights-of-Ways" (2020). Graduate Theses, Dissertations, and Problem Reports. 7892.

https://researchrepository.wvu.edu/etd/7892

This Thesis is protected by copyright and/or related rights. It has been brought to you by the The Research Repository @ WVU with permission from the rights-holder(s). You are free to use this Thesis in any way that is permitted by the copyright and related rights legislation that applies to your use. For other uses you must obtain permission from the rights-holder(s) directly, unless additional rights are indicated by a Creative Commons license in the record and/ or on the work itself. This Thesis has been accepted for inclusion in WVU Graduate Theses, Dissertations, and Problem Reports collection by an authorized administrator of The Research Repository @ WVU. For more information, please contact researchrepository@mail.wvu.edu. 
Assessment of Unmanned Aerial Systems and lidar for the Utility Vegetation Management of Electrical Distribution Rights-of-Ways

\author{
Matthew R. Walker \\ Thesis submitted to the \\ Davis College of Agriculture, Natural Resources, and Design \\ at West Virginia University \\ in partial fulfillment of the requirements for the degree \\ of \\ Master of Science in \\ Forest Resources Management \\ Gregory A. Dahle, Ph.D., Chair \\ Mike Strager, Ph.D. \\ Timothy A. Warner, Ph.D. \\ Aaron Maxwell, Ph.D.
}

Division of Forestry and Natural Resources

Morgantown, West Virginia

2020

Keywords: Unmanned Aerial Systems (UAS), Lidar, Utility Vegetation Management (UVM)

Copyright 2020 Matthew R. Walker 


\title{
Abstract \\ Assessment of Unmanned Aerial Systems and lidar for the Utility Vegetation
}

\author{
Management of Electrical Distribution Rights-of-Ways
}

\author{
Matthew R. Walker
}

Utility Vegetation Management (UVM) is often the largest maintenance expense for many utilities. However, with advances in Unmanned Aerial Systems (UAS; or more commonly, "drones") and lidar technologies, vegetation managers may be able to more rapidly and accurately identify vegetation threats to critical infrastructures. The goal of this study was to assess the utility of Geodetics' UAS-lidar system for vegetation threat assessment for $1.6 \mathrm{~km}$ of a distribution electric circuit. We investigated factors which contribute to accurate tree crown detection and segmentation of trees from within an UAS-lidar derived point cloud, and the factors which contribute to accurate tree risk assessment. The study adapted the International Society of Arboriculture's (ISA) tree risk assessment methodology to the application of remotely sensed tree inventory. We utilized the lidar detected and segmented tree crowns for tree risk analysis based upon each tree's height, elevation, and location in relation to the electrical infrastructure. The individual tree detection and segmentation results show that our canopy type parameter and the routine used for field- and lidar-derived tree matching to have the largest effect on the classification agreement of field and lidar derived datasets. The Threat Detection classification also demonstrated a significant effect due to our canopy modeling parameter, where single canopy models possessed higher average Kappa agreement statistic and divided canopy models detected a larger number of threats on average. Ultimately, our best model was capable of the correct detection, segmentation, matching, and classification of half of the field trees which were determined to be vegetation threats. 


\section{Acknowledgments}

The completion of this thesis would not have been possible without the support, guidance, or aid of those around me. While it is impossible for me to give each and every one of you my fullest and heartfelt thanks here, I would like to recognize a selection of people from whom I sought assistance at different points throughout this process.

I would like to thank my academic advisor, colleague, and friend, Dr. Greg Dahle, for the many thought-provoking conversations and car rides, for helping me find employment after my undergraduate degree, and after which, providing me the avenue to return to WVU as his graduate student. During our time working together, Greg continually found patience with me and provided me the leeway to pursue my own research interests.

I would like to thank my academic committee: Dr. Tim Warner, Dr. Aaron Maxell, and Dr. Mike Strager. It was my pleasure to work with, Dr. Warner, his constant curiosity and inquisitiveness were an aid every time I felt my own motivations waiver. Likewise, I can only hope that my sense of wonder for my field of study is as steadfast at the twilight of my own career. Again, thank you Dr. Warner. Furthermore, Dr. Maxwell's kindness is only matched by his genius, of which I'm sure he'll deny, yet his teachings provided me the basis to expand my own knowledge and complete this project. Without his aid this project would have been impossible. Again, thank you Dr. Maxwell. Lastly, I'd like to thank Dr. Strager, for trailblazing my path forward as researcher and natural resource manager. His work to integrate spatial decision making in natural resources over the past two decades is a source of inspiration of mine. Again, thank you Dr. Strager.

Additionally, I would like to thank some other instrumental WVU faculty members and teams. I'd like to thank my friend and colleague Dr. Samuel Taylor, for his support of my entire graduate career, but particularly for his support of the research conducted herein. Likewise, I'd like to thank Dr. Taylor's professional affiliate WVU's Energy Institute for their monetary support of this research project through the O'Brien Energy Research Fund and, to express my thanks as a West Virginian to the Albert O'Brien and family for their positive impact on West Virginia and WVU through the O'Brien Energy Research Fund. Furthermore, I'd like to extend my gratitude to Dr. Paul Kinder and his lab, WVU's Natural Resource Analysis Center. In particular, I'd like to thank Sam Behringer and Lucas Kinder. Without the help of these three 
individuals this research project would have quite literally, not gotten off the ground. Lastly, I'd like to extend a very special thanks, to Dr. Ida Holásková, my supervisor during the past year for her patience, interest, willingness to bring me on as her assistant. She astounds me weekly with her humility, depth and breadth of knowledge, and willingness to learn. Again, thank you all.

Finally, I'd like to thank those who are not university affiliated but played significant roles in this project. First, I'd like to thank my previous supervisor, A. Todd Rovello, for his assistance in obtaining me the permission to conduct my research on First Energy Mon Power rights-of-ways and for all the days he and I spent hiking and auditing rights-of-ways and the conversations while doing so. Next, I wish to extend a thanks to First Energy Mon Power for the opportunity to conduct this research and would like to thank the rest of Mon Power's Distribution Forestry team. I'd also like to extend a special thanks to Dr. Francesco Pirotti of Univerità Degli Studi di Padova for his aid when I cold called him for assistance with how to conduct the accuracy assessment contained herein. Likewise, I'd like to Dr. Jean-Romain Roussel, of the Université Laval, for his work compiling many of the most cutting edge lidar tools pertaining to forestry into one $\mathrm{R}$ package. Thank you all, this project would not have been possible without your assistance.

Additionally, I wish to express the utmost gratitude for my numerous colleagues from Biomechanics Week 2019 and in particular, NASA's Matt Melis, the Morton Arboretum's Dr. Jake Miesbauer, Rutgers University’s Dr. Jason Grabosky. Furthermore, Mark and Janet Hoenigman deserve a very special thanks, for their facilitation of such a wonderful event and for bringing me and other researchers into their home for fine food and wonderful discussions. I'd also like to thank the Bartlett Tree Expert Company and Dr. Tom Smiley, for the annual invitation to their research lab in Charlotte, NC during my time working with Dr. Dahle. Each of these events were rejuvenating and after each event, I always found myself re-endowed with creative energy to put forth in my own research. Again, thank you all.

Finally, I'd like to thank the many friends and family who have witnessed my struggles as well as my triumphs during this project. I would not have been able to persevere had it not have been for you all. Again, thank you everyone. 


\section{Table of Contents}

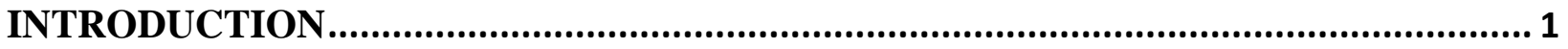

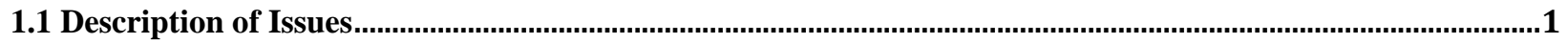

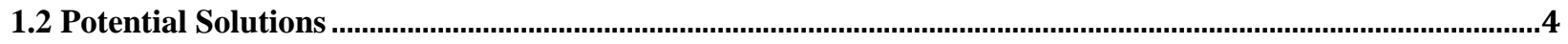

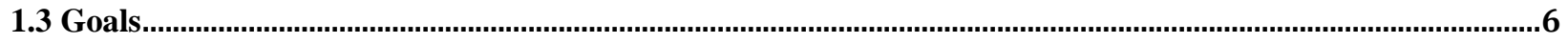

1.4 Significance

1.5 Document Structure

LIKELIHOOD OF FAILURE OF TREES: A LITERATURE REVIEW ...................... 11





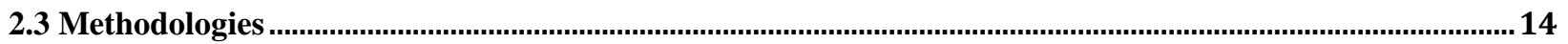

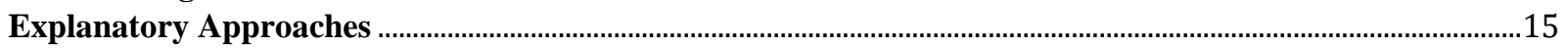

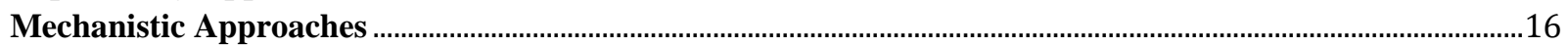

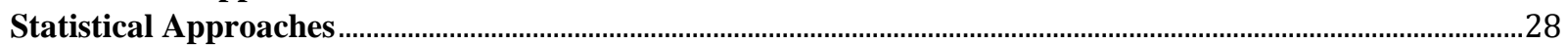

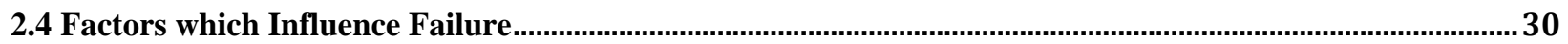

Stems

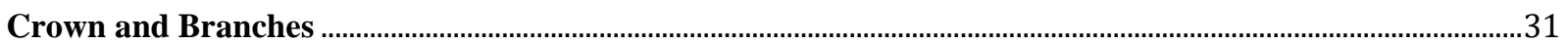

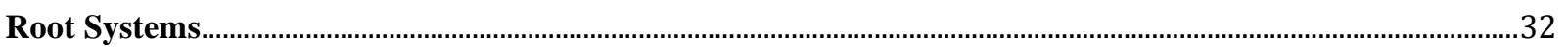

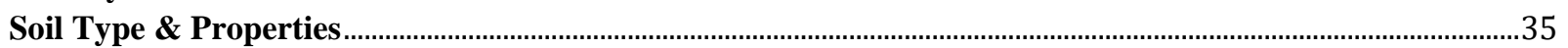

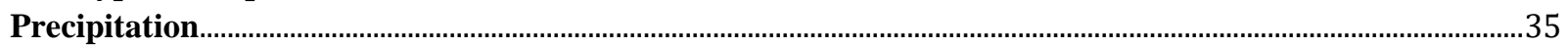

Wind

2.5 Conclusion

LITERATURE REVIEW OF UAS AND LIDAR WITH APPLICATION TO UVM....... 40

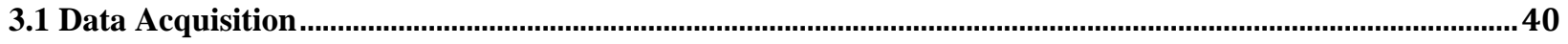





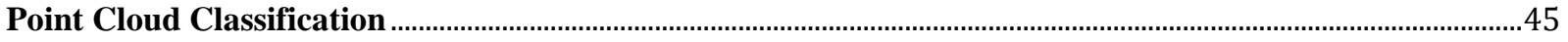

Individual Tree Crown (ITC) detection and segmentation ...................................................................................4

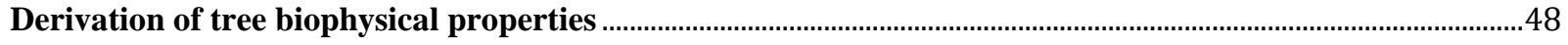

ASSESSMENT OF UAS-LIDAR SYSTEM FOR UVM................................................. 50

4.1 Introduction 


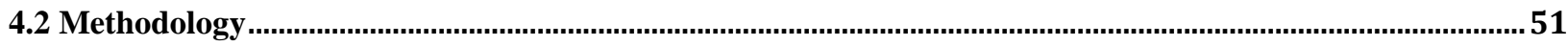

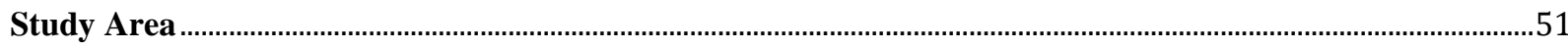

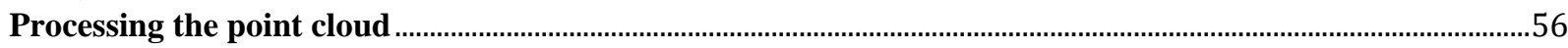

Individual Tree Crown Detection and Segmentation ………………….................................................................... 71





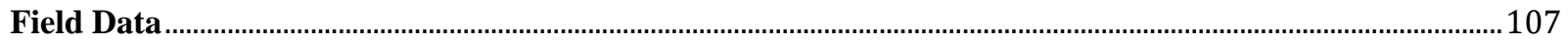



DEM \& Pole Locations ................................................................................................................................... 114

Individual Tree Crown Detection and Segmentation ......................................................................................118



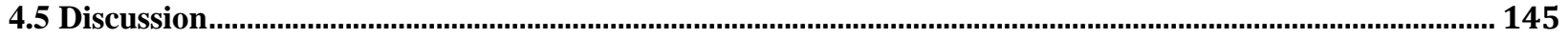

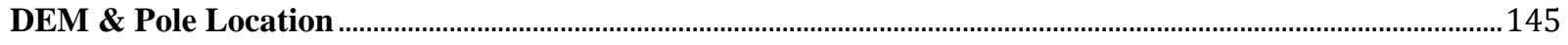

Individual Tree Crown Detection and Segmentation Metrics …………………..................................................148

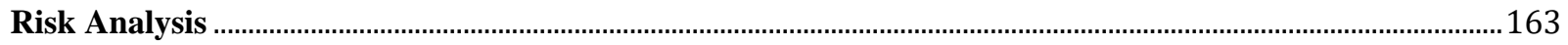

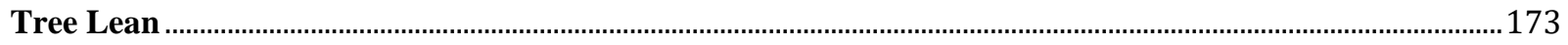



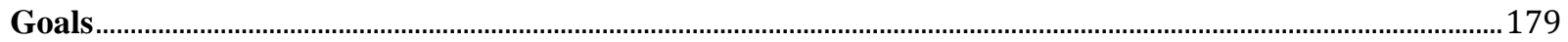



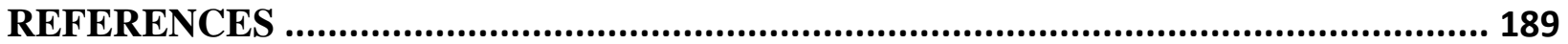

\section{FIGURES}

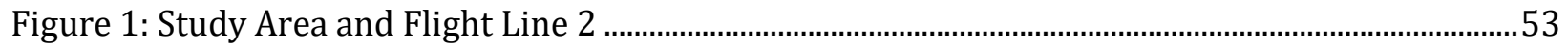

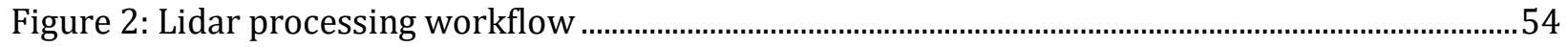

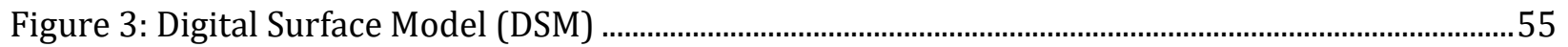

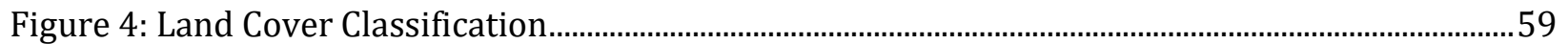

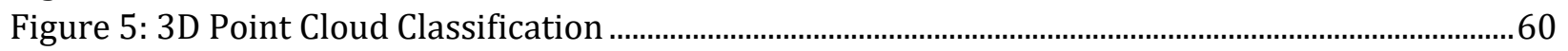

Figure 6: Areas of Interest with Field plots ............................................................................................61

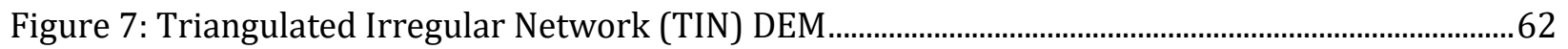

Figure 8: Inverse Distance Weighted K-nearest neighbor DEM................................................................63

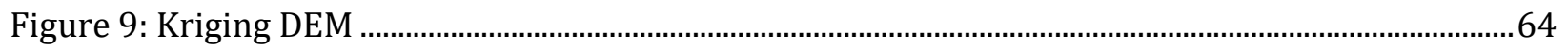



Figure 11: Canopy Height Model (CHM) for TIN DEM normalized, full density point cloud (PC1) ....66 Figure 12: Canopy Height Model (CHM) for the TIN DEM normalized, decimated point cloud (PC 3)

Figure 13: Canopy Height Model (CHM) for the KNN DEM normalized, full density point cloud (PC2)

Figure 14: Canopy Height Model (CHM) for the KNN DEM normalized, decimated point cloud (PC4)

.

Figure 15: Comparisons of Canopy Height Model differences.......................................................................

Figure 16: Issues in Individual Tree Detection and Segmentation ............................................................73

Figure 17:Individual Tree Crown Detection and Segmentation Result, example 1 1...............................74

Figure 18: Individual Tree Detection and Segmentation Result, example 2 .............................................75 
Figure 19: Individual Tree Detection and Segmentation Result, example 3..........................................76

Figure 20: Individual Tree Detection and Segmentation Result, example 4 ..............................................77

Figure 21: Pole Detection and Segmentation..........................................................................................



Figure 23: Differences in tree base estimation methods ......................................................................... 84

Figure 24: Nearest point on the electric line from tree base locations.....................................................87

Figure 25: Matched Trees and NERC FAC-003 Category 2 \& 3 Vegetation Threats ................................88 Figure 26: The distributions of the Likelihood of and Likelihood of Impact measures for all matched trees and the Likelihood of Impact measure for matched trees determined to be vegetation threats

Figure 27: Recode formula for Likelihood of Failure Measure to ISA's Likelihood of Failure

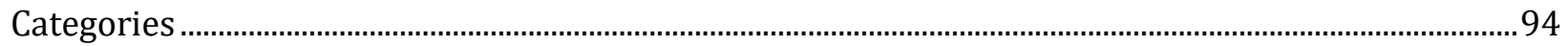

Figure 28: Likelihood of Failure Classification....................................................................................................95

Figure 29: Likelihood of Failure classification for Matched Trees .....................................................................96

Figure 30: Likelihood of Impact Classification formula .................................................................................97

Figure 31: Likelihood of Impact Measure Calculation.................................................................................99

Figure 32: Likelihood of Impact classification for trees identified as within the striking distance of

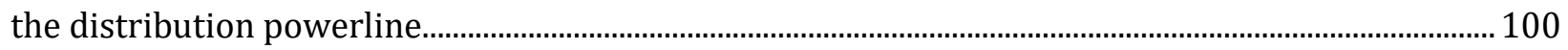

Figure 33: Likelihood of Impact classification for matched trees …….................................................... 101

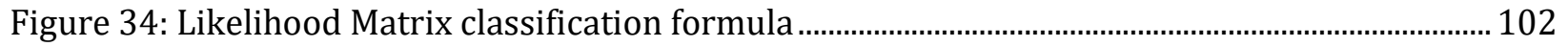

Figure 35: Electrical grid customer count model...................................................................................... 103

Figure 36: Consequences of Impact classification formula ..................................................................... 104

Figure 37: Final Risk Rating classification formula .................................................................................... 104

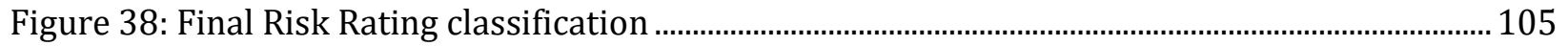

Figure 39: Final Risk Rating classification of matched trees................................................................... 106

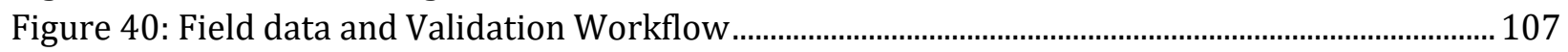

Figure 41: Field Tree Metrics: Distributions of Height, DBH, and Elevation ........................................ 111

Figure 42: Distributions of Field Tree Likelihood of Failure and Likelihood of Impact Measures... 112 Figure 43: Field Tree Metrics: ISA Tree Risk Assessment Classification of Field Trees determined to be Threats.......................................................................................................................................... 113

Figure 44: DEM comparison: Connecting Letters Report....................................................................... 116

Figure 45: Pole Location Differences....................................................................................................... 117

Figure 46: Tree Detection and Matching Rates by matching method and matching feature.............. 121

Figure 47: F-score, Precision, and Recall by matching method and matching feature........................ 121

Figure 48: Commission Error Rate and Esyn et al.'s (2015) accuracy index by matching method and

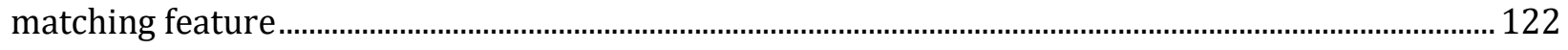

Figure 49: Kappa of Agreement for Matching and Tree Location RMSE by matching method and

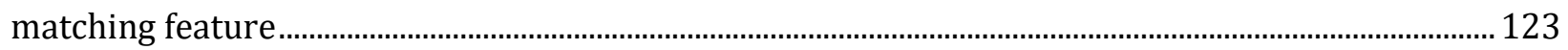

Figure 50: Matched Tree Height R-square, correlation, and RMSE by matching method and matching

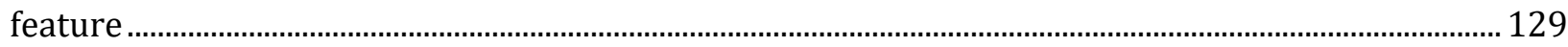

Figure 51: Matched Tree DBH R-square, correlation, and RMSE by matching method and matching

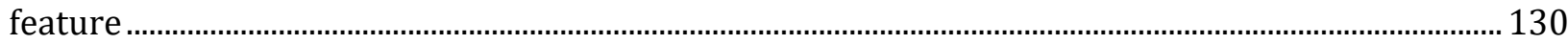

Figure 52: Distributions of Kappa Agreement Statistics for each risk analysis classification by



Figure 53: Distribution of Kappa Agreement Statistics for each ISA tree risk assessment classification. 
Figure 54: Contingency Table Analysis for Consequences of Impact ...................................................... 143

Figure 55: Undetected Threats: Field Measures............................................................................................. 152

Figure 56: Undetected Threats: Risk Measures .................................................................................... 153

Figure 57: Undetected Threats: Distributions for the ISA's Tree Risk Assessment Classifications. 154

Figure 58: Unique, Matched Field Trees ................................................................................................ 155

Figure 59: Percent of Field Tree Threats correctly detected, segmented, matched, and classified by

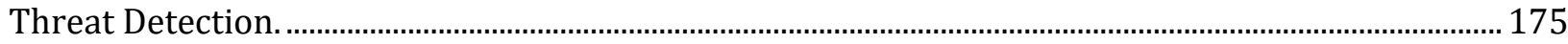

Figure 60: Contingency analysis for the TIN DEM normalized, decimated, divided canopy model, using the XY tree base approximation method and the $4.5 \mathrm{~m}$ matching method, which was selected


Figure 61: Projected tree matching rate necessary to correctly detect, segment, match, and classify

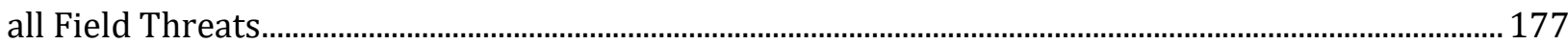

\section{TABLES}

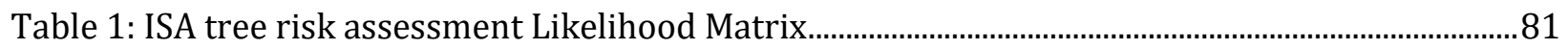



Table 3: Fit Statistics for "New Predicted DBH \#1" .............................................................................................91

Table 4: Prediction Statistics for "New Predicted DBH \#1"......................................................................92

Table 5: Individual Tree Detection and Segmentation accuracy metrics for all models ...................... 124 


\section{Chapter 1}

\section{Introduction}

\subsection{Description of Issues}

Utility Vegetation Management (UVM) along electric rights-of-ways (ROWs) is the single largest maintenance cost faced by many electric utilities, with costs of $\$ 2$ - 10 billion annually in the United States alone (Guggenmoos 2003; Guggenmoos 2007). In the aftermath of the Northeast Blackout of 2003, in which a lack of vegetation management played a key role, the Energy Policy Act of 2005 allowed the Federal Energy Regulation Commission (FERC) to enact the North American Electric Reliability Corporation (NERC) policies and guidelines for electric systems operations as federal regulatory standards.

Most notably, Standard FAC-003, titled "Transmission Vegetation Management”, requires a "Transmission Vegetation Management Plan" (TVMP) which designates a plan to maintain safe and reliable electric power through the management of vegetation on and adjacent to electrical transmission right-of-way (ROW). While unplanned outages were more common along distribution electric systems, FAC-003 does not apply to distribution-level UVM. Even so, most electric utility companies have some sort of UVM plan for their distribution system. These plans are generally compliant with State-level regulations. Yet, due to variance in reporting methods between states, the reports are often not comparable.

While the reports do not utilize the same reporting methods or terminology, many electrical utilities cite "tree caused/related" or "vegetation caused/related" as their most common type of unplanned disruption (Guggenmoos 2003; Guggenmoos 2007; Guggenmoos 2011). Of these tree or vegetation related disruptions on the distribution system, most are attributed to "fallins", trees outside the ROW which were tall enough to fall into the ROW and across or through 
the electric conductor (NERC FAC-003; Guggenmoos 2003; Guggenmoos 2007; Guggenmoos 2011). Furthermore, most unplanned disruptions occur on the distribution system, with vegetation-related outages generally regarded as the leading cause (Guggenmoos 2003, Guggenmoos 2007; Ituen et al. 2008).

Two major factors likely contribute to vegetation-related outages: the lack of easement authority and/or the offending vegetation is located outside the ROW and therefore is not managed by the utility (Miller et al., 2015). These unplanned outages have a direct effect on electric reliability and grid stability, as well as the US economy. An Electric Power Research Institute (EPRI) study estimated that US business sectors lost between $\$ 104$ to \$164 billion in 2001 due to power outages in either the transmission or distribution grid (Lineweber \& McNulty 2001).

Additionally, the sheer scope of the current electrical grid in the United States compounds on the issues of non-standardized reporting methods, state versus federal regulations based on voltage, and state to state variability in state-level regulations. To demonstrate, the North American Electric Transmission System consists of all electric lines carrying $35 \mathrm{KV}$ and higher, stretches approximately 476,398 linear miles, and requires the management of an estimated 8.6 - 11 million acres (Warwick et al. 2016; Miller et al. 2015). While the associated electrical distribution system is comprised of approximately 6,332,236 linear miles of lines carrying less than $35 \mathrm{KV}$ (Warwick et al., 2016). Thus, if the distribution is patrolled on a five (5) year cycle, each year would require over one million miles of line to be patrolled annually.

The goal of UVM is to provide safe and reliable electric power, and multiple specialized personnel play integral roles in the maintenance and monitoring of vegetation conditions. Utility foresters manage on a larger scale, usually associated with a service area, region, or across an 
entire utility. Meanwhile, utility arborists are tasked with monitoring vegetation conditions on the ROW, as well as, monitoring and auditing line clearance contractors. Line clearance contractors are the largest segment of the UVM industry, and fill numerous, varied roles, including qualified line clearance arborists, heavy machine operators, and groundmen. Standardized tree risk assessment protocols are beneficial to UVM in that they provide the most consistent qualitative assessment of a tree's likelihood of failure, likelihood of impact, and overall risk (Smiley et al. 2017, Goodfellow 2020). Yet, utility foresters and arborists do not often inspect off-ROW vegetation due to constraints such as accessibility of time, which leaves many trees off-ROW trees unmonitored or with limited monitoring. While shortening pruning cycles has been found to both decrease the number of disruptions and decrease the time and cost to prune individual trees the use additional vegetation management resources across a utility's grid can be costly and result in excessive pruning, exacerbating poor tree health along a utility's rights-of-ways (Goodfellow 2000, Miller et al. 2015, Guikema et al. 2006, Radmer et al. 2002, Kuntz et al. 2002, Kuntz et al. 2001). Furthermore, foot patrol for visual inspection of distribution electric corridors often relies upon an individual forester's subjective interpretation of vegetation conditions, and as such may not be accurate or comparable between different individual utility foresters or arborists (Ferguson et al. 2012, Koeser 2017).

In addition to the previously described issues, there is significant difficulty in assessing the likelihood of failure of a given tree within a determined time. Even so, the International Society of Arboriculture's (ISA) current tree risk assessment best management practices utilize a Tree Risk Assessment Qualified (TRAQ) arborist's qualitative assessment of the likelihood of failure of a given tree within a defined duration of time (Smiley et al. 2017). While a quantitative 
assessment of the likelihood of failure would be ideal, it is computationally intensive, and the effects of major contributing factors are difficult to assess (Ciftci et al. 2013, James et al. 2014).

When an anticipated load exceeds the moment capacity of the tree, the tree is expected to experience failure. This definition of "failure" doesn't require tree fall or outwardly visible structural failure. Trees may crack or split, develop a ring shake, or experience the severing of roots without tree fall. These are all examples where the tree has been pushed past its point of elasticity and are considered initial failures. Final failure may be complete and catastrophic if portion of the tree falls onto a target, or complete and undamaging if the tree hangs up in a neighboring tree. During the inspection of vegetation in and along electric ROWs, it can be difficult to assess or recognize trees with elevated likelihood of failure, as structural issues may not be outwardly visible (Dahle et al. 2006).

From 2001 forward, the American Society of Civil Engineers (ASCE) has released an Infrastructure Report Card every four years which details the current infrastructure conditions and needs within the US. For the 2017 report, the US scored a "D+" under the "Energy" category, due to aging infrastructure and a lack of resilience in the face of severe weather events. A list of recommendations provided by the ASCE included, "Promote usage of remote sensing and inspection technologies to lower the cost of energy system monitoring; focus operation and maintenance spending on highest-risk system components” (Energy 2017).

\subsection{Potential Solutions}

The application of emerging remote sensing technologies, particularly lidar and Unmanned Aerial Systems (UAS), to distribution vegetation management may prove to be faster, more accurate, and ultimately, more cost-effective than current and traditional UVM methods. 
Targeted prescriptive management of individual trees and problematic areas may be prioritized, due to the rapid and accurate identification of vegetation threats (Miller et al. 2015). Most electric utility companies already use some form of Geographic Information System (GIS) to map their transmission and distribution electric systems. In the past decade, some utilities have augmented their GIS through the integration airborne lidar data as a tool to verify compliance of the United States' transmission system with federal regulations. These lidar systems have generally been mounted on small aircraft, such as helicopters and airplanes, and have relatively high costs of operation. Yet, with the advent of affordable UAS and the falling costs lidar technology, the regulation, mapping, and modeling of the distribution electric system could very well be the next step in developing a more reliable grid. Additionally, these technologies may allow for more precise and cost efficient UVM approaches. At the moment, there is a research gap specific to the application of UAS-based lidar for distribution-level utility vegetation management. Despite this, there are growing related fields of research in UAS-based lidar for forest inventories (Jaakkola et al. 2010; Wallace et al. 2012; Wallace et al. 2014; Wallace et al. 2016) and airborne lidar for transmission electrical system maintenance (Ituen et al. 2008; Kim \& Sohn 2013; Ko et al. 2012).

The second edition of the tree risk assessment best management practices specifically addresses utility management (Smiley et al. 2017) and just recently the utility tree risk assessment best management practices (BMP) was released (Goodfellow 2020). These BMPs assess overall risk using a matrix that categorizes the likelihood of failure, likelihood of impact, and the consequences of the impact. Identifying trees with elevated likelihood of failure and elevated likelihood of impact can be a challenge for any vegetation management program, Especially in rugged, remote areas with countless trees growing just outside the ROWs. Utilizing 
UAS and lidar in tandem may allow for the rapid identification of trees with elevated risk that are growing both in and alongside the distribution ROWs.

Furthermore, lidar data has the advantage of being able to be stored and reviewed or utilized again at a later date (Ferguson et al. 2012). This will allow for GIS-based change detection methodologies to be used between scans, and could be used to audit tree removal, calculate vegetation growth rates, and to monitor natural tree failure in the utility forest. The latter, monitoring tree failure in the utility forest would be useful for further refinement of predictive individual tree failure models and UVM risk management tools.

\subsection{Goals}

This study aims to assess the abilities and application of an UAS-based lidar system for UVM of the electric distribution system. We are particularly interested in the identification and assessment of vegetation risks for improved tree risk mitigation and electric reliability. It is the intention of this project to use data acquisition methods developed from UAS-based lidar for forest inventory combined with data processing approaches from aerial lidar for transmission electric vegetation management, to determine whether UAS-based lidar is a viable tool for distribution-level UVM. Our final product will provide a recommendation on where vegetation management activities should be concentrated to most effectively mitigate risk. The intended result is less tree-caused outages and improvements in electrical reliability and grid stability.

The primary focus of the project is to utilize a UAS-based lidar system to identify

potential NERC FAC-003 category 2 and 3, "fall-in" vegetation risks and assess the risk of each to electrical service and infrastructure. Secondarily, we will identify potential NERC FAC-003 category 1, "grow-in" vegetation risks. 
First, we will utilize a combination of diameter at breast height (DBH) derived from tree crown volume and height as a surrogate model for likelihood of a tree failure. Arboricultural "post-storm" literature suggests that these measures are the best predictors of tree failure which coincide with tree biometrics which are both simply derived and reasonably accurate (Kane 2008, Gardiner et al. 2008, Peterson 2007). However, further research into tree risk assessment, and particularly calculation of likelihood of failure, from UAS-based aerial lidar-derived biometrics and other geospatial data is necessary to standardize and better understand tree risk modeling at the grid scale.

Second, this project will investigate:

1. The effect of Digital Elevation Model interpolation methods on individual tree crown detection and segmentation and risk analysis.

2. The effect of point cloud decimation on individual tree crown detection and segmentation and risk analysis.

3. The effect of tree base location approximation method on individual tree detection and the identification and risk analysis of NERC Category $2 \& 3$ vegetation risks.

4. the Likelihood of Impact of NERC Category $2 \& 3$, "fall-in" vegetation risks on the electric lines.

The above list comprises the key factors for this project's final goal of providing a recommendation on where vegetation management activities should be concentrated to most effectively mitigate vegetation risks. The intended result is fewer tree-caused outages and improvements in electrical reliability and grid stability. 


\subsection{Significance}

This project represents a couple firsts in the scientific literature, including: the first demonstration of UAS-lidar system for distribution-level utility vegetation management and the first Likelihood of Impact assessment of individual trees on the electrical infrastructure from a lidar point cloud. Additionally, this project contributes to several research areas, including: the generation of Digital Elevation Models (DEMs) from UAS-based lidar point clouds (Zhang et al. 2003, Wallace et al. 2016), individual tree crown detection and segmentation from lidar (Li et al. 2012, Wallace et al. 2012, 2014, \& 2016; and Hamraz et al. 2016), and the geospatial prediction of individual tree failure (Gardiner et al. 2000, Ancelin et al. 2004, and Peltola et al. 1999).

This project was funded by a NIFA McIntyre Stennis grant (WVA00108) and a West Virginia University Energy Institute's O'Brien Energy Research Fund seed grant, and the TREE Fund Utility Arborist Research Fund grant \#19-UAA-01. The O’Brien Energy Research Funds must demonstrate a benefit to the energy sector within West Virginia. West Virginia poses an interesting set of challenges for vegetation managers; the state is primarily forested, rural, and rugged, with long expanses of inaccessible ROWs. West Virginia's need for more effective and modern vegetation management strategies were made clear in 2014, when the WV Public Service Commission (PSC) ordered electric companies in WV to begin a six-year transition to a four year, end-to-end, cycle-based vegetation management strategy for electrical distribution lines. In addition, a cost-recovery mechanism was implemented to offset the cost of PSC approved vegetation management programs.

To best serve West Virginians, as electric consumers and citizens of the state, more efficient and flexible vegetation management strategies should continue to be developed through the integration of UAS, lidar, and risk management concepts to utility vegetation management 
operations. Furthermore, benchmarks and standards for vegetation management operations should be developed and implemented such that different UVM strategies can be assessed and selected among.

This research project demonstrates the benefit of interdisciplinary cooperation of natural resource management, arboriculture, remote sensing, and geospatial analytics. As both West Virginia and the United States attempt to modernize aging electrical infrastructure, interdisciplinary collaborations will become ever more crucial to address the large, expensive, and complex issues faced by the state and the nation. Due to these circumstances, we anticipate further interest in related research projects, such as: the use of change detection for the optimization of UVM operations, utilization of UAS for post-storm damage assessment, and the use of UAS-based lidar for the inspection of electrical equipment, among others. These related research needs further emphasize the importance of funding opportunities such as O'Brien Energy Research Fund seed grant to the state, industry, and individual researchers.

\subsection{Document Structure}

This document includes two literature reviews, Chapter 2 which pertains to the likelihood of failure in trees; and Chapter 3 which details UAS and lidar technologies and data processing techniques with particular interest in the remote sensing of electric ROWs. Chapter 4 is our assessment of an UAS-based lidar for the UVM of electrical distribution ROWs, including: a description of the study area, the methodology for the research project, a description of the acquired dataset, presentation of the project's results and validation, and a discussion of the outcomes. The chapter concludes with a review of the project in its entirety and suggest potential avenues for future research. It is anticipated that Chapter 2 will be submitted to the journal of 
Arboriculture and Urban Forestry. Additionally, Chapter 3 and 4 are intended to be submitted for publication to a geospatial journal. 


\section{Chapter 2}

\section{Likelihood of Failure of Trees: a literature review}

\subsection{Introduction}

Trees cause electric outages primarily through two methods: first, by failing structurally such that the tree strikes electrical infrastructure; and second, by growing into the electrical conductor thus providing an unintended ground (Appelt \& Goodfellow, 2004). Most vegetationrelated electrical outages are attributed to trees which exist outside of the ROW and possess the height necessary to fall into or through the electrical conductor, these trees are known as "fallins".

Many trees fail along the stem or at the soil-root plate due to wind loading, since it is the most prevalent force plants must deal within the terrestrial environment (Niklas 1992, Guggenmoos 2003, Guggenmoos 2007, Guggenmoos 2011, and Guggenmoos \& Sullivan 2012). We refer to the process of wind-induced stem breakage or uprooting, as "windthrow". Windthrow is a dynamic process; there have been several methods proposed in the literature for predicting the likelihood windthrow (Coutts, 1986, James et al. 2014, Dahle et al. 2017). For examples, see: Baker 1995, Peltola et al. 1999, Ciftci et al. 2014, Kamimura et al. 2016, Suzuki et al. 2016, Virot et al. 2016, Yan et al. 2016, and Kamimura et al. 2017. Snow and ice also cause loading on trees, though these types of loading are generally regarded as static (James et al. 2014, Dahle et al. 2017). For examples of studies examining snow and ice loads, see: Peltola et al. 1999, Gaffrey \& Kniemeyer 2002, and Lulely \& Bond 2006. In addition to external forces, decay can deteriorate wood strength, particularly decreasing the moment capacity of the stem or branch, thus heightening the likelihood of failure (Ciftci et al. 2013). 
To differentiate stem breakage and uprooting as two different types of windthrow, we will refer to these as different modes of failure. Furthermore, material properties can influence the mode of failure of a tree, which has implications for assessing the likelihood of failure, particularly in branches (Dahle et al. 2006, Dahle \& Grabosky 2010). In earlier windthrow literature, it was customary to limit the concept of "wind damage risk" to the likelihood of a particular percentage of trees experiencing uprooting or breakage (Gardiner et al. 2008). Gardiner et al. (2008) suggested a more appropriate term might be "wind damage probability modelling" (Gardiner et al. 2008). More recent research has explored the possibility of predicting the probability of windthrow for individual trees (Ciftci et al. 2014, Kamimura et al. 2016).

Currently, utility vegetation managers need tools for predicting windthrow risks and knowledge of the necessary management prescriptions to reduce the risk of windthrow damage to the utility's electric infrastructure. Risk accounts for both the likelihood of an event and the consequences caused by that event (Smiley, Matheny, \& Lily, 2017). Qualitative assessments are commonly used by decision makers to assess windthrow risks (Millet et al. 1987, Mitchell, 1998, Gardiner et al. 2008). Empirical models have been developed to assess the probability of windthrow of individual trees or the probability of an expected proportion of stand damage based on tree and stand attributes in forest stands, plantations, and seaside shelterwoods (Peltola et al. 1999, Gardiner et al. 2008, Suzuki et al. 2016). Nevertheless, a better understanding of the likelihood of failure of individual trees and the relationships governing tree failure and vegetation-related outages would allow for significant advances in the risk management of electric distribution lines (Appelt \& Goodfellow, 2004).

Participating researchers at the 2010 Tree Biomechanics Summit at the Morton Arboretum identified five areas of focus for future research of tree biomechanics, the first of 
which was "assessing the likelihood of failure in trees" (Dahle et al., 2014). This review will focus on key studies involving the likelihood of failure of trees. We'll begin with a description and discussion of failure in trees. Thus will be Followed by an examination of methodologies that have been used to assess tree failure and a review of factors which influence tree failure. We will then conclude with a discussion of emerging technologies and the application of existing models to the datasets derived from these technologies.

\subsection{Defining Likelihood of Failure}

Current tree risk assessment methods, generally utilize a professional arborist's qualitative assessment of the likelihood of failure of a given tree within a defined duration of time (Smiley et al. 2017). While quantitative assessments of the likelihood of failure of trees have been completed, the process is computationally intensive, and the effects of the contributing factors are difficult to evaluate (Ciftci et al. 2013, James et al. 2014). Simply stated, the theoretical likelihood of failure of a tree can determined by the moment capacity of the tree, the anticipated loads the tree will experience, and the anticipated weather-related phenomena which the tree will experience (Dahle et al. 2017). Yet, there is sparse information available for the load bearing capacity of trees, the anticipated load trees intercept, and the site and environmental factors that affect failure (Dahle et al. 2017, James et al. 2014, Dahle et al. 2014).

The inspection of vegetation in and along electric ROWs for UVM is difficult due to the fact that trees with elevated likelihood of failure, such as those with significant internal decay or structural issues, may not be observable or obvious from a foot patrol's visual inspection (Dahle et al. 2006). Additionally, utility foresters may not be able to assess each tree individually, due to lack of access to the location of the tree base or time constraints. Trees can also begin the process 
of failing, initial failure, without incurring full structural failure, or "final failure". Thus, assessing the number of trees that have experienced final failure and have fallen within a specified time period will be easier than attempting to assess the number of trees which have experienced initial failure. This is particularly true of remotely sensed data, where the presence or absence of a given tree over a time series of images or scans may be detectable. However, there does not currently exist methods to remotely assess whether a given tree has experienced initial failure.

Furthermore, the UVM industry stand to benefit from change detection techniques and remote sensing technologies, such as lidar data. With successive scans of the same area, one should be able to visualize vegetation differences along ROWs. In particular, the presence of new vegetation or absence of previously present vegetation should be obvious. Change detection methodologies would also aid in calculating vegetation growth rates, perhaps down to the individual tree or stem. Additionally, remote sensing and change detection could provide a robust set of tools to help monitor a large number of trees over time, which would potentially be useful in the calculation of the likelihood of failure of trees. However, due to limitations of current remote sensing technologies, the likelihood of tree failure derived from a change detection study would be limited to the detection of tree fall, and thus, final failure.

\subsection{Methodologies}

Several techniques have been proposed in the literature to assess the likelihood of windthrow of trees (Baker 1995, Peltola et al. 1999, Ciftci et al. 2014, Kamimura et al. 2016, Suzuki et al. 2016, Virot et al. 2016, Yan et al. 2016, and Kamimura et al. 2017). Kabir et al. (2018) separates these research techniques into three key methodological groups: explanatory 
approaches, mechanistic approaches, and statistical approaches, and our review will follow this grouping. In the following section we will discuss each of the three methodological approaches, including an in-depth discussion of different methodologies within mechanistic approaches. Furthermore, each of the biomechanical methodologies mentioned have benefits and drawbacks, and all have aided in augmenting the existing knowledge base.

\section{Explanatory Approaches}

Explanatory approaches assess the relationship of tree failure and a variety of physical or geographical parameters, such as tree species, diameter at breast height (DBH), soil characteristics, or mode of failure (Kabir et al. 2018). The primary methodology within explanatory approaches is referred to a "post-storm study", where, after a storm-event, standing and failed trees are examined to discern patterns in measurable physical properties or geographic characteristics.

Francis and Gillespie (1993) related wind gust speed to tree damage, where the maximum damage category was uprooting. They found their uprooting category to be independent of both DBH and gust speed, while stem breakage decreased with increasing diameter and was also independent of wind gust speed (Francis \& Gillespie 1993). Additionally, they conclude that large trees are at greater risk than small trees, which supports Reilly (1991).

Peterson (2007) observed consistent influence of tree diameter and species on tree failure due to tornado blowdowns. He observed that windthrow occurrence increased with tree diameter, and that uprooting was more common among trees of smaller size classes (Peterson 2007).

Kane (2008) examined tree failure after a windstorm in Brewster, Massachusetts. He too found that the likelihood of failure increased with trees of greater $\mathrm{DBH}$ and height. Yet, the different failure rates were not able to explain variation among species (Kane 2008). 
Furthermore, Kane states that the study did not factor in exposure, which is known predictor of damage (Kane 2008; Gardiner et al. 2008).

Lastly, explanatory studies are limited in that they typically utilize parametric analyses, such as, logistic or linear regression; and/or use ' $\mathrm{R}^{2}$ ' as indicator of predictive accuracy, thus leading to over-fitting (Kabir et al. 2018).

\section{Mechanistic Approaches}

The fundamental premises of tree biomechanics are: trees cannot violate the laws of physics, trees are mechanical objects, and tree size and shape are limited by biomechanical constraints (Niklas 1992, James et al. 2014, de Langre 2008, Spatz \& Bruechert, 2000; James et al. 2014). Therefore, engineering and physical methods are reasonable methodologies to attempt to understand the structural properties of trees and how they interact with the environment (James et al. 2014). Dependent upon the line of action of a force, trees will experience stress in the forms of tension, compression, and shear when subjected to bending and torsion loading (Dahle et al. 2017). Furthermore, wood and most plant material are described as viscoelastic, and may behave in non-linear fashion during mechanical loading (James et al. 2014, Miller 2005). Additionally, trees, being living organisms, may adapt or change their material properties as a result of age, growing conditions, or loading regimes (James et al. 2014, Plomion et al. 2001, Woodrum et al. 2003, Read \& Stokes 2006, Dahle \& Grabosky 2010).

A tree's material properties are factors which affect its load-bearing capacity (Dahle et al. 2017). The two most commonly reported material properties are, the elasticity modulus $(E)$ and modulus of rupture $(M O R)$, and are used to describe a material's stiffness and maximum loadbearing capacity, respectively (Dahle et al. 2017, Burgert 2006). Additionally, material properties can influence the mode of failure of a tree (Dahle et al. 2017). 
There is a large body of literature describing of wood properties, see: (Panshin and De Zeeuw 1980, Haygreen and Bowyer 1982, Kollman and Cote 1984, Bodig and Jayne 1993, Dahle et al. 2017). Despite this, applying measured wood properties to living trees may not accurately estimate a given individual tree's material properties, due to the large variability of material properties of wood with age, growing conditions, genetics, moisture content, and location in an individual (Zobel \& van Bujitenen 1989, Clair et al. 2003, Dahle and Grabosky 2010b, Kretschmann 2010, Dahle et al. 2017).

In addition, the values of $E$ and $M O R$ vary longitudinally, tangentially, and radially within an individual tree, often decreasing axially with trunk height and or branch length (Niklas 1992, Lundstrom et al. 2008, Kretschmann 2010, Dahle \& Grabosky 2010b, Dahle et al. 2017). Juvenile wood often has lower values of $E$ and $M O R$ than mature wood, and the proportion of juvenile wood to mature wood can influence $E$ and $M O R$ (Lundstrom et al. 2008, Dahle \& Grabosky 2010b, Dahle et al. 2017). This generally allows for younger, more flexible, distal parts of the tree crown to reconfigure in the wind, and more mature, rigid, proximal tree parts, such as the stem, structural branches, and structural roots, to resist increased loading from selfweight and wind-induced bending and torsional moments (Niklas 2002, Clair et al. 2003, Lundstrom et al. 2008, Dahle \& Grabosky 2010b, Dahle et al. 2017). Also, E and MOR have been found to be positively correlated with wood density and specific gravity and, have been observed to increase as moisture content decreases below fiber saturation point (Dahle et al. 2017). Lastly, while $E$ and $M O R$ have been modeled for stems and branches, there is little literature in regard to root wood or wound wood (Lundstrom et al. 2008, Dahle and Grabosky 2010b, Dahle et al. 2017). 
Tree form, or architecture, also plays a strong role in a tree's load-bearing capacity (Dahle et al. 2017, Dahle \& Grabosky 2009). The length and diameter of stems and branches and the direction of loading affects the bending and torsional moments induced by the load (Dahle et al. 2017). When an equivalent load is applied, longer branches and stems endure greater bending and twisting moments than shorter ones (Dahle et al. 2017, Dahle \& Grabosky 2012). The loadbearing capacity is related to cross sectional area and second moment of area, which is known as the moment of inertia (I) (Dahle et al. 2017, Burgert 2006). The effect of diameter on the loadbearing capacity of stems and branches is non-linear, such that $I$ is proportional to the fourth power of diameter (Dahle et al. 2017). Therefore, it is not surprising that explanatory studies have observed a positive linear relationship between failure and tree diameter (Dahle et al. 2017). In addition to material properties, allometry, the study of relationship of body size to shape, has been used to mechanistically assess trees. A common allometric measurement used to assess stability in trees is the relationship between length and diameter (Dahle \& Grabosky 2009). An early allometric assessment of trees, Greenhill's (1881) study on the critical buckling height of trees, demonstrated a structural model of a tree, where the tree is considered as a pole and a static analysis was utilized to calculate how tall a tree could grow before it would buckle under its own weight (Spatz 2000). McMahon (1975), proposed three models for the allometric assessment of failure but, no model has been found to fit all trees (Dahle \& Grabosky 2009, Dahle et al. 2017). Yet, for gymnosperms and understory rainforest trees, the geometric similarity model appears to work; the static stress model appears true for mature pines; and the elastic similarity model can be applied to many angiosperms (Dahle \& Grabosky 2009, Dahle et al. 2017). A full review of these models can be found in Dahle and Grabosky (2009). 
Greenhill's (1881) simple pole model for trees has been the conceptual basis for both static and dynamic analyses of trees (Spatz 2000, James et al. 2014). To demonstrate the difference, if a force is applied in a static manner, it will result in a deflection of a certain magnitude. If the same force is applied in a dynamic or cyclic fashion, at a certain frequency, the deflection may be greater than the deflection caused by the equivalent static force (James et al. 2014). For this reason, dynamic analysis considers the inertial forces of mass (m), the elastic forces $(\mathrm{k})$, and the damping forces $(c)$, whereas static analysis only considers the elastic forces (k) (James et al. 2014).

\section{Static Pull Tests}

Static pull tests utilize a rope or cable attached to the tree to apply a measured load, followed by an assessment of the strength of the trunk and root plate (Wessolly 1991, Clair et al. 2003, Peltola et al. 2006, Dahle et al. 2017). This research methodology is commonly at the forefront of tree likelihood of failure research. While this methodology operates under the assumption windthrow is a dynamic process, it develops static equations for wind load analysis, which may or may not accurately model actual wind loading regimes (Niklas 1992, Hale et al. 2010, Dahle et al. 2017).

Two types of sampling methods are utilized in static pull test research, destructive and non-destructive. Destructive sampling can yield maximum loads at failure in the trunk or rootplate (Peltola et al 2006, Dahle et al. 2017). non-destructive sampling can provide a quantitative assessment of the uprooting resistance of a tree's root system (Wessolly 1991, Dahle et al. 2017).

The close correlation between bending moments necessary to cause change in soil-root plate inclination and the maximum resistive moment of the root system during the uprooting process makes root plate rigidity a good indicator for anchorage strength (Vanomsen 2006, 
Smiley 2008, Lundström et al. 2009). This relationship has been established in the literature for several species and has been used to extrapolate failure loads (Lundström et al. 2009, Sani et al. 2012, Smiley et al. 2014).

Much of the work on static load tests has been concentrated on the relationship between stress and strain within the proportional limit of the stress-strain curve, known as the "elastic range". While this may be useful when studying primary failure, researchers should also consider the impact of plastic strains, those strain beyond the elastic range, which often result in permanent but not always fatal damage, on the load carrying capacity of trees (Dahle et al. 2017). In summary, although static pull tests and static biomechanics have aided our understanding of how trees handle loads, they use simplifying assumptions which may or may not accurately represent real world loading schemes (Niklas 1992, Hale et al. 2010, Dahle et al. 2017). For examples of static pull test studies, see: Kane 2014 or Peterson \& Claassen 2013).

\section{Dynamics}

In an attempt to better represent these real-world loading schemes, researchers have utilized dynamic analysis methods. Three different approaches are commonly used to assess the dynamic behavior of trees (Clough \& Penzien, 1993; James et al. 2014). The first, is the lumpedmass procedure, where mass is assumed to be concentrated at a discrete point (James et al. 2014). The second, utilizes generalized displacements for a uniformly distributed mass, with the trunk treated as a beam (James et al. 2014). Lastly, the Finite Element Method (FEM) utilizes complex computer modeling (James et al. 2014).

The lumped-mass procedure, which assumes the mass is concentrated at a discrete point as it oscillates dynamically, is a simplification of the actual dynamic process of windthrow, since inertial forces only develop at the mass points (James et al. 2014). Even so, this method has been 
used to develop spring-mass-damper models for trees as a single mass, or as a complex system of coupled masses that represent the trunk and branches (Milne, 1991, Miller 2005, James et al. 2014).

The uniform distributed mass method considers a tree as a beam or column, with its mass uniformly distributed along its length and a fourth-order partial differential equation has been used to study the oscillations and damping of woody and non-woody plants (Gardiner et al. 2000; Spatz 2000; Moore \& Maguire 2008; James et al. 2014).

The Finite Element Method combines features of both the lumped-mass and uniformly distributed mass procedures (Sellier et al. 2006, Moore \& Maguire 2008, Theckes et al. 2011, Ciftci et al. 2013, James et al. 2014). FEM divides a structure, in this case a tree, into an appropriate number of elements, beams, whose sizes may vary, and the ends of which, nodes, become the generalized coordinate points. An advantage of FEM is that complex wind loading scenarios can be modeled (James et al. 2014). Yet, FEM's reliability is limited by its requirements of multiple accurate, empirical measurements peculiar to the individual tree and its loading conditions (James et al. 2014).

All models used for dynamic analysis of trees make assumptions and may not accurately represent the complex dynamics of trees (Moore \& Maguire 2004). Models must account for the damping and dynamic contribution of branches (de Langre 2008, Rodriguez et al. 2008, James et al. 2014). Additionally, trees require multi-degrees of freedom, or multimodal analysis, to model dynamic interactions between the branches and trunk, and literature is lacking on how these interactions take place (Sellier et al. 2006, de Langre 2008, Rodriguez et al. 2008, James et al. 2014). 
Damping dissipates energy, and thus reduces the amplitude of oscillation through the frictional forces of aerodynamic drag and collisions, and internal, viscoelastic forces (Milne 1991, James et al. 2006, James et al. 2014). Damping forces are considered velocity dependent, and are most effective around the natural frequency, while having little effect at lower and higher frequencies where the inertia of a tree's mass is the dominant effect (James et al. 2014). Furthermore, damping is usually not well understood in vibrating structures or in nature (Clough \& Penzien 1993, James et al. 2014). The effect of damping may be non-linear, thus potentially it may result in a higher level of complexity than seen in most dynamic models to this point (James et al. 2014).

Multimodal response in branched structures occurs when several coupled masses, branches, oscillate in a complex manner, with in-phase and out-of-phase responses such that several modal swap responses are possible (Rodriguez et al. 2008 James et al. 2014). The branches, with their individual oscillation responses, are connected to another oscillating mass, the stem, resulting in a coupled response of the combined masses (Rodriguez et al. 2008, Theckes et al. 2011, Ciftci et al. 2013, James et al. 2014). This branched multimodal method has been applied to trees, such that the branches are considered as coupled masses that oscillate on the trunk, which itself is oscillating (Rodriguez et al. 2008, Theckes et al. 2011, Ciftci et al. 2013, James et al. 2014). These complex models of trees that represent the dynamic oscillations of branches have used either a multiple spring-mass-damper model or the FEM approach (Rodriguez et al. 2008, Theckes et al. 2011, Ciftci et al. 2013, James et al. 2014).

Furthermore, where multimodal response occurs, a damping effect known as "mass damping” may also occur (James et al. 2014). Mass damping was described by Den Hartog (1956) and has been defined for trees (James et al. 2006). Mass damping occurs when the 
branches sway together or against each other, in-phase and out-of-phase, respectively (de Langre 2008; Theckes et al. 2011; James et al. 2014). Mass damping allows for the dissipation of forces exerted by wind on tree crowns in a non-destructive fashion. Additionally, trees may also dissipate wind energy through a mechanism called "multiple resonance damping" (Spatz et al., 2007), “multiple mass damping” (James et al. 2006), or "branch damping” (Spatz \& Theckes 2013; James et al. 2014).

recent literature suggests branches influence the dynamic behavior of trees to a greater extent than can be explained by their additional mass (Moore \& Maguire 2004, James et al. 2014). The dynamic response of trees to wind loading is greatly influenced by the size and form of the tree, and in part due to the dynamics of branches (James et al. 2014). James et al. (2014) concluded with the following list of gaps in the literature surrounding the dynamics of trees:

1. Recommendations for pruning open-grown trees to reduce wind damage.

2. Further study of the dynamic contribution and the damping effects of branches.

3. Models of open-grown trees accounting for the multimodal branch response.

4. Observation of tree failure under actual wind conditions.

5. Further study of energy transfer from wind to tree.

6. Further study of torsional forces and loads experienced by trees.

\section{Predicting tree failure with mechanistic models}

Gardiner et al. (2008) published a review of predictive, mechanistic models of wind damage to forests. These models attempt to capture the physical processes involved in tree uprooting or failure typically through a two-step process. The initial stage is to calculate the above-canopy "critical wind speed" (CWS) required to cause windthrow within a forest (Gardiner et al. 2008). The second stage is to use some assessment of the local wind climatology 
to calculate the probability of such a wind speed occurring at the geographic location of the trees (Gardiner et al. 2008). They termed this probability of damage, the "risk of damage" (Gardiner et al. 2008). The approaches used to calculate the CWS and the local wind climate may vary between models the different predictive models (Gardiner et al. 2008).

These predictive mechanistic models attempt to approximate the CWS of trees based on the anticipated wind-related forces and the counteracting and combined resistive forces of their roots and stem (Gardiner et al. 2008). When predicting the CWS, the resistance to overturning is based upon correlations between the bending moment required to cause windthrow and stem weight or root-soil plate weight (Gardiner et al. 2008). While the resistance to breakage of a tree is related to the diameter of the stem and the tree species and must be greater than the bending moment required to exceed the MOR or stem failure will occur (Gardiner et al. 2008). "These relations can be simplified to state that the stem volume best predicts the resistance to uprooting, whereas dbh3 best predicts resistance to stem breakage” (Quine \& Gardiner 2007, Gardiner et al. 2008).

The second stage of the mechanistic modeling of windthrow risk to trees is predicting the probability of the CWS being exceeded (Gardiner et al. 2008). The primary method to predict the local wind climate is to use the airflow model, Wind Atlas Analysis and Application Program (WAsP) (Mortensen et al. 2005; Gardiner et al 2008). Although in settings with more complex terrain or wind climates the use of Weibull parameters from highly accurate weather forecast data may be required for accurate airflow modelling (Mitchell et al. 2007; Gardiner et al. 2008).

The GALES model utilizes tree height, diameter, current tree spacing, soil type, cultivation, drainage, and tree species to determine the CWS (Gardiner et al. 2008). GALES was originally designed to calculate the CWS at $10 \mathrm{~m}$ above the zero-plane displacement height for 
even-aged conifer monocultures. To consider mixed species stands the simulation must be run for each species in turn and all trees in the stand must be considered to be of that species (Gardiner et al. 2008). Gales can be utilized to calculate the risk at any distance from a newly created edge and for any size of upwind gap (Gardiner et al. 2008). For existing edges, the risk is considered constant from the edge due to the effects of adaptive growth by trees (Telewski 1995, Gardiner et al. 2008). Additionally, GALES requires tree pulling data, MOR for the green timber of the tree species of interest, and descriptive measures of the crown characteristics (Gardiner et al. 2008). When using GALES, it has been found that an increase of the predicted CWS by an additional fixed value of $1 \mathrm{msec}^{-1}$ improves the accuracy of the model's predictions (Gardiner et al. 2008).

The HWIND model was developed by Peltola et al. (1999) for the description of the mechanistic behavior of monocultures of Scots Pine, Norway Spruce, and birch under wind and snow loading (Peltola et al. 1999, Gardiner et al. 2008). While originally designed for calculations of the CWS of trees at newly created edge of stands, HWIND has now been adapted for the calculation of CWS at different distances from the upwind gap and for different sizes of upwind gap (Gardiner et al. 2008). HWIND predicts the mean CWS over a 10-minute time period at $10 \mathrm{~m}$ above ground level (Gardiner et al. 2008). This model requires knowledge of tree species, tree height, DBH, stand density, distance to the stand edge, and gap size (Gardiner et al. 2008). HWIND, like GALES, is sensitive to any inaccuracies of the inputs, especially DBH, which determine the amount wind load a tree can experience before failure and the expected amount of wind load a tree will experience (Gardiner et al. 2008). Thus any inaccuracy can have a significant influence on the predicted CWS (Gardiner et al. 2008). 
The FOREOLE model developed by Ancelin et al. (2004), was the first attempt to contend with complex stand structure within predictive mechanistic models (Gardiner et al. 2008). FOREOLE assumes an empirical wind profile within the canopy and calculates the horizontal wind loading on each individual tree (Gardiner et al. 2008). Reasonable agreement between the predictions made by GALES, HWIND, and FOREOLE have been noted when compared (Gardiner et al. 2008). While FOREOLE has yet to be entirely validated, its predicted CWSs have aligned with the wind speeds required to cause damage to trees (Gardiner et al. 2008).

To quantify wind loading, GALES may use either a "roughness method", where a windinduced stress distribution of trees in a forest is calculated; or a predicted wind profile within or at the forest front (Gardiner et al. 2008). In contrast, HWIND and FOREOLE both utilize only the latter method (Gardiner et al. 2008). An early limitation of CWS-based models was that they were originally built to represent the risk to a "mean tree" within a stand, not to consider the risk posed to individual trees (Gardiner et al. 2008). However, recently Suzuki et al. (2016) determined CWS for individual trees, as well as demonstrated a quantitative risk management evaluation for individual trees (Suzuki et al. 2016).

Most of these CWS-based models are limited because they do not account for variations in wind from different directions (Gardiner et al. 2008). While Ancelin et al. (2004) demonstrated a first attempt to deal with complex stand structure, their approach has not yet been validated against data from complex stand structures (Gardiner et al. 2008). Additionally, Wellpott \& Gardiner (2006) suggested that Ancelin et al.'s (2004) approach is not a realistic representation of wind loading on individual trees (Gardiner et al. 2008). A possible alternative approach to modeling wind risk of individual trees is to make use of the competition indices 
developed for predicting growth conditions of individual trees within stands, which Achim et al. (2007) demonstrated are extremely well correlated to the wind loading of individual trees within a mature Sitka spruce plantation (Gardiner et al. 2008). Furthermore, while GALES and HWIND attempt to capture some site characteristics (e.g. soil type, drainage, rooting depth), FOREOLE solely uses the wind and tree measurements, and does not take into account site characteristics.

While, Schelhaas et al. (2007) demonstrated a method to incorporate tree-tree interactions into a CWS model, the development of better predictive mechanistic models through accounting for spatial variability, tree to tree interaction, and the propagation of windthrow at the individual tree level in homogenous or multi-structured stands, is a critical need to the literature (Gardiner et al. 2008).

To become more than research tools, these predictive mechanistic models must be incorporated into forest management systems in ways that are useful and practical (Gardiner et al. 2008). Yet currently, due to the need of numerous, precisely measured parameters these models are not practical in many cases. While these tools have not been widely utilized in practice, Gardiner et al. (2008) suggests that first, their operation must be simple and interpretation of the results routine (Gardiner et al. 2008). Future research into predictive mechanistic models should integrate decision support tools to simplify each model's operation, such that the requirements are a hierarchical set of questions on the characteristics of the trees and site, and outputs are different levels of risk low to high (Kamimura et al. 2008, Gardiner et al. 2008). Moreover, the integration of other remote sensing data and additional GIS layers to enhance location specific conditions may be useful for the prediction of tree failure. 


\section{Statistical Approaches}

Statistical approaches, much like explanatory approaches, utilize geographic characteristics and physical properties of trees as variables to aid in the prediction of windthrow (Kabir et al. 2018). However, instead of utilizing a single statistical tool, such as linear regression, statistical approaches examine the relationships of the measured properties through the lens of multiple statistical tools to see which tool best predicts windthrow (Kabir et al. 2018). Example of such properties include Generalized Linear Models (GLMs), Monte Carlo simulation (MC), classification and regression trees (CART), Random Forests (RF), and Artificial Neural Networks (ANN);

Ciftci et al. (2014) utilized a Monte Carlo-based methodology for the prediction of individual tree failure. Their study attempted to quantify the probability of failure of two maple trees in Massachusetts. Although one of the first and more novel methods for the prediction of likelihood of failure of individual trees, this study is limited in that it was computationally intensive and not well suited for the large datasets that would be associated with trees along electric distribution ROWs (Ciftci et al. 2014).

Kamimura et al. (2016) developed a logistic regression models and utilized a GALESbased model for individual tree failure from one storm at an Aquitaine forest in southwestern France, then validated the model against the next storm at that location. Their results suggested that GALES was capable of predicting wind damage risk of trees on certain soils, while their statistical models were not able to be generalized to other locations or storm events. (Kamimura et al. 2016).

Kabir et al. (2018) used the covariates location, height, DBH, existence of severe defects, whether or not a tree had been pruned, and whether or not a tree had been removed in the immediate proximity of the tree in question to demonstrate that tree failure can be statistically 
estimated. Kabir et al. (2018) utilized several statistical tools, including a GLM with a Bernoulli response, CART, a multivariate adaptive regression spline (MARS), ANN, Naïve-Bayes Classifier, boosting, RF, and an ensemble model of RF and boosting. The ensemble model of boosting and Random Forest yielded the best prediction accuracy for estimating the failure probability of trees for their data set (Kabir et al. 2018).

Kabir et al. (2018) was a novel approach to predicting windthrow of individual trees and contributed to the literature, primarily by demonstrating the potential predictability of tree failure using statistical models. Additionally, Kabir et al. (2018) demonstrated the first use of multiple statistical tools for the prediction of windthrow, including: CART, MARS, ANN, Naïve-Bayes Classifier, boosting, RF, and an ensemble model of RF and boosting. However, the results of this study cannot be used to estimate tree failure probabilities for either other storms at the study site or at other locations because the models implemented included data from only one storm, at the one study site (Kabir et al. 2018).

Thus far in likelihood of failure research, most statistical analyses have limited their statistical tools to linear or logistic regression (Kabir et al. 2018). Nevertheless, Ciftci et al. (2014) and Kabir et al. (2018) have demonstrated the utility of other statistical tools. Additionally, most studies are not able to be generalized as the models developed only apply to one location or one storm due to the fact that the models were not validated in subsequent locations or storms. Yet, Kamimura et al. (2016) developed models, both statistical and GALESbased, in one storm and validated them against a second storm, at the same location. Furthermore, studies utilizing more sophisticated statistical tools and multiple storm or multiple location model validation methodologies are needed and desired additions to the literature. 


\subsection{Factors which Influence Failure}

Across all methodologies certain factors which contribute to tree failure have been illuminated. In this section we will discuss the factors that have been related to tree failure across all methodological approaches, including: tree stems, tree crowns and branches, root systems, soil type and properties, precipitation, and wind.

\section{Stems}

A tree's stem and crown characteristics have been found to dictate how trees resist loads, whether from self-weight, wind, snow, or ice (Niklas 2000, Peterson \& Claassen 2013, Niklas \& Spatz 2000). Post-storm study literature has suggested failures are more likely as tree size and wind speed increase (Duryea et al. 2007b). Kane (2008) observed an increase in likelihood of failure of trees with a greater diameter and taller trees. Peterson (2008) also observed that as tree diameter increased so did the risk of tree failure. Additionally, Kabir et al. (2018) found that the probability of failure for a tree increased for tall trees, though the height used to determine "tall" was not provided. Additionally, Kabir et al.'s (2018) model found that trees with smaller DBH were more likely to experience failure, which is incongruent with most current literature (Kabir et al. 2018). Kabir et al. (2018) also examined the levels of importance of different tree properties in their models, with regards to their level of influence on the likelihood of tree failure. Height and DBH were shown to have large influences on a model's predictions, whereas the removal of nearby trees was indicated to have a relatively small effect on a model's prediction (Kabir et al. 2018).

Despite the general correlation of increased tree size and increased likelihood of failure, multiple studies have found that tree size and wind gust speeds by themselves cannot explain the variation in failure rates for different tree species (Francis \& Gillespie 1993, Kane 2008). Yet, 
despite the unexplained variation within species, simplified methods for estimation of uprooting and stem breakage have been described (Kane 2008, Gardiner et al. 2008). In addition, Lundstrom et al. (2007) found that $75 \%$ of the variation of the turning moment in the soil-root plate was explained by tree mass, trunk mass, trunk diameter, or tree height, either alone or in combination, during static loading (Lundstrom et al. 2007).

Decay is a major component of the likelihood of failure of a given tree (Smiley, Matheny, \& Lily, 2017). Decay causes moment capacity loss in loss in tree branches and stems (Ciftci et al., 2013, Dahle et al. 2006), and the severity and location of decay are the factors which determine the effect of decay on likelihood of failure (Luley et al., 2009). Kane's (2008) study found that most trunk failures (76\%) involved a defect, Though currently the detection of decay through remote sensing means does not appear to be feasible, and as such the full relationship of decay and likelihood of failure will not be reviewed here. Instead, see Dahle et al. (2014), Ciftci et al. (2013), or Kane (2008) for a more complete review of the relationship of decay and the likelihood of failure.

\section{Crown and Branches}

Crown size and shape has been generally found to play a significant role in how trees resist wind, snow, and ice loads (Niklas \& Spatz 2000, Gaffrey \& Kniemeyer, 2002). Wind induced stress varies along the length of the stem and is partly influenced by the crown shape and size, as well as, stem taper (Niklas \& Spatz 2000). Furthermore, stem taper, canopy shape, and canopy size have a more significant effect on wind-induced stem stress intensities than the shape of the wind speed profile (Niklas \& Spatz 2000).

Pruning recommendations for the mitigation of wind-induced tree failure is a topic of debate in the literature, and definitive pruning recommendations have yet to be made. However, 
Gaffrey \& Kniemeyer (2002) found that a crown volume reduction of 50\% was found to reduce sail area by $18 \%$, which caused a stress reduction of $15-24 \%$ (Gaffrey \& Kniemeyer 2002). Yet, in the same study, an asymmetric crown reduction resulted in a mid-crown increase in stress of up to $25 \%$, which may have implications for UVM ground-to-sky trimming techniques (Gaffrey \& Kniemeyer 2002). Furthermore, Kane (2008) found that pruning did not reduce a tree's overall likelihood of failure.

The literature does suggest that the time of year or season can account for up to a $40 \%$ difference in probability of failure, particularly in deciduous trees, due to differences of leaf-off and leaf-on wind, snow, and ice load interception (Ciftci et al. 2014). Additionally, thinning of an individual tree may help prevent snow and/or ice damage to that tree, but in turn may change wind regiments and make wind-induced failure of neighboring trees more likely (Peterson \& Claassen 2013, Peltola et al. 1999; Kane 2008).

\section{Root Systems}

Root systems play a vital role in tree stability, and the stability of a tree at least partly depends upon its root spread, root architecture, and root plate development (Dahle et al. 2017). Yet, the most important region of a tree's root system, in regard to tree failure, appears to be the soil-root plate (Dupuy et al. 2007, Ji et al. 2007, Tobin et al. 2007, Ghani et al. 2009, Dahle et al. 2017). Smiley (2008) found that trenching at a distance less than twice the trunk diameter reduced anchorage strength by more than $15 \%$, and if lateral roots were severed at the trunk base the anchorage strength was reduced by roughly $35 \%$ (Dahle et al. 2017). Furthermore, during static pull tests, trees were observed to not return to upright if inclined past a certain degree, generally above 1-2.5 degrees, at the tree's base (Sinn 1990). After such an inclination, the stiffness of the root-soil plate was found to be decreased and the same result was found to be 
achievable through cyclic loading to one degree inclination at a tree's base (Rogers et al. 1995, Vanomsen 2006, Lundstrom et al. 2009).

The soil-root plate of younger trees was found to have a greater degree of rotation at maximum resistance, and the degree of rotation at maximum resistance is expected to vary with tree age class, root architecture, and soil structure (Crook \& Ennos 1996, Stokes 1999). Furthermore, as trees grow their root system develops greater strength and, in response to loading over time, root shape may be altered (Dahle et al. 2017). This adaptive growth may decrease the likelihood of overturning during a loading event (Dahle et al. 2017). Additionally, when trees do uproot, a consistent relationship between tree diameter and the size or volume of the root pits and mounds has been observed (Peterson 2008). Also, root failures were observed to be more likely at sites where nearby trees had been removed prior to storms (Kane 2008). Yet, it is difficult to determine how the interactions of neighboring tree removal, the associated wind regiment change, hypothesized elevated stress levels at the soil-root plate of the remaining tree, soil properties at that location and time, and likelihood of tree failure relate to one another.

A given tree's mode of failure appears to be, at least, partly dependent upon its physical properties. Niewenhuis \& Fitzpatrick (2002) suggested that tree diameter was weakly related to mode of failure and had observed increased uprooting versus stem breakage for trees of smaller size classes (Nieuwenhuis \& Fitzpatrick 2002). Others also observed that young trees, as well as those in the largest size classes, experience less stem breakage and are more likely to uproot (Peterson 2007, Peterson \& Claassen 2013). Putz et al. (1983), Asner \& Goldstein (1997), Gardiner et al. (2000), and Peterson (2008) all observed some influence of wood strength on a tree's mode of failure (Peterson 2008). 
Multiple findings have suggested greater vulnerability of conifers and early successional species, but the support is weak (Peterson 2008, Kabir et al. 2018). When a species tends to possess traits for both deep rooting and strong wood, they are generally resistant to windthrow; for example, Acer sacchurum (Peterson 2008). In addition, wood strength was observed to have some influence on the risk of treefall ("final failure") and the mode of failure but was generally not significant on its own (Peterson et al. 2008, Putz et al. 1983, Asner \& Goldstein 1997, and Gardiner et al. 2000). Furthermore, wood strength seems more indicative of the mode of failure, where trees with stronger wood are more likely to experience uprooting and trees with weaker wood are more likely to experience stem breakage (Peterson 2008). This relationship could explain how a variable for "tree species" may partially capture that particular species' general wood properties, while partially confounding the results due to the effect of that individual tree's crown and root architecture.

In conclusion, a tree's biophysical properties, including stem, crown, and root characteristics have been found to dictate how trees resist loads, whether from self-weight or wind, snow, or ice loads (Niklas 2000, Peterson \& Claassen 2013, Niklas \& Spatz 2000). Additionally, these biophysical properties have a more significant effect on wind-induced stem stress intensities than the shape of the wind speed profile (Niklas \& Spatz 2000). Furthermore, the literature has suggested failures are more likely as tree size increases (Duryea et al. 2007b, Peterson 2008, Reilly 1991, Kane 2008). Root systems also play a vital role in tree stability and, decay is a major component of the likelihood of failure of a given tree (Smiley et al. 2017). A given tree's mode of failure also appears to be, at least, partly dependent upon its physical properties. Gardiner et al. (2008) simplified these relations as, "stem volume best predicts the resistance to uprooting, whereas $d b h^{3}$ best predicts resistance to stem breakage." 
Furthermore, the linear relationships between critical bending moment and stem mass or stem dimensions obtained from static tree pull studies have been reported for many species, and stem mass was generally found to be the best predictor of windthrow (Nicoll et al. 2006, Gardiner et al. 2008). The regressions which relate critical bending moment to stem dimensions, as well as crown drag to branch mass, are robust, and the tight relationships suggest that trees follow some set of consistent principles of biomechanical design (Gardiner et al. 2008). While Wessolly's generalized tipping curve has been criticized, the increasing probability of windthrow with tree size appears general, with the maximum resistive moment of a given tree's anchorage occurring at angles between two (2) and six (6) degrees for the mixed-species stands of eastern North America (Coutts 1986, England et al. 2000, Vanomsen 2006, Lundström et al. 2007).

\section{Soil Type \& Properties}

As previously discussed, trees depend upon their root systems for structural support. Additionally, soil type and soil conditions are factors which affect the load-bearing capacity of a tree's root system (Dahle et al. 2017). The most crucial region appears to be the soil-root plate, and its depth is particularly important in sandy or clay soils (Dupuy et al. 2007, Ji et al. 2007). In Smiley's (2008) trenching study, the side of the tree where the roots were cut had an influence when soil was water saturated, but not under dry conditions. This demonstrates the importance of soil conditions (e.g. type, texture, and moisture content) on the process of windthrow and how important soil plays an integral role in the soil-root plate and tree stability (Dahle et al. 2017).

\section{Precipitation}

Rain alone does not often cause tree failure, though saturated soils exacerbate wind caused failure rates (Peterson, 2007). Thinning (pruning) of an individual tree helps prevent snow and/or ice damage but may have repercussions related to wind regiments and the wind 
exposure of neighboring trees (Peterson \& Claassen 2013, Peltola et al. 1999, Kane 2008). Snow and ice loads cause the static loading of trees and may help explain the vast difference in likelihood of failure of deciduous trees, due to phenological differences of leaf-on load interception and leaf-off load interception (Ciftci et al. 2014, James et al. 2014, Dahle et al. 2017). Furthermore, when snow or ice loads are intercepted in tandem with wind loading, elevated likelihoods of failure are to be expected but there is little empirical evidence detailing the relationship of combined wind and snow/ice loads. Yet, some research has incorporated both wind and snow/ice loads into their models (Peltola et al. 1999, Gaffrey \& Kniemeyer 2002, Lulely \& Bond 2006, Niklas \& Spatz, 2000, and Ciftci et al. 2014).

\section{Wind}

The literature has suggested failures are more likely as tree size and wind speed increase (Duryea et al. 2007b). Niklas (2000) suggested that wind is likely the most common causal factor of tree failure and, has been described as the most prevalent dynamic force on trees in the terrestrial environment (Niklas 1992, James et al. 2014). Wind gusts may initiate more failures than a constant wind speed, since gusts cause additional crown displacement (Milne 1988). Additionally, changes in the local wind regiment, through the removal or failure of neighboring trees in the stand will result in higher likelihood of failure of remaining trees due to increased exposure to wind forces (Peltola et al. 1999, Kane 2008, Peterson \& Claassen 2013). Furthermore, stem taper, canopy shape, and canopy size also possess a more significant effect on wind-induced stem stress intensities than the shape of the wind speed profile (Niklas \& Spatz 2000).

The fluid pressure of wind increases with the square of wind velocity (Francis \& Gillespie 1993). Thus, the severity of wind damage to trees can be explained by relatively small 
increases in wind speed (Francis \& Gillespie 1993). There are a multitude of ways to express wind data, including: scales, such as the Beaufort Scale; and wind speed (James et al. 2014). Wind speeds have been reported in a variety of units (e.g., miles per hour, kilometers per hour, knots etc.). Furthermore, instantaneous wind speeds are rarely available and average wind speed may be calculated over either 10-minute or one-hour intervals (James et al. 2014). Wind gust speed is described as an average wind speed, though taken over a three-second interval (Holmes 2007, James et al. 2014). The lack of consistent reporting methods and measures of wind can be an obstacle to disseminating knowledge for practical tree risk management (Cullen 2002b).

Predictive mechanistic modeling studies have shown the critical wind speed (CWS) for a vast number of tree species to exist between $36-234 \mathrm{~km} / \mathrm{h}$, with many species failing by roughly $180 \mathrm{~km} / \mathrm{h}$ (Suzuki et al. 2016, Virot et al. 2016). Francis \& Gillespie (1993) observed that wind induced tree damage was not present below $\sim 60 \mathrm{~km} / \mathrm{h}$, damage increased rapidly as gust speeds increase from $60-130 \mathrm{~km} / \mathrm{h}$. Then, beyond $130 \mathrm{~km} / \mathrm{h}$ variability in damage increased dramatically (Francis \& Gillespie, 1993). Additionally, the wind speed necessary to cause tree failure will vary depending on tree species, growth pattern, and location (James et al. 2014). Yet, trees generally cannot weather violent storms with mean wind speeds exceeding 108 $\mathrm{km} / \mathrm{h}$ at the top of the canopy, for a period of 10 minutes, without sustaining some amount damage (Peltola, 1996a, James et al. 2014). Canham \& Loucks (1984) postulated that as the severity of damage increases, the differences between species, size, and other factors diminish, until a threshold at which most trees over a certain diameter fail. This idea is one with which Francis and Gillespie (1993) unknowingly concurred, positing their own idea of "storm buildup". Storm build-up describes a process where there exists a wind speed at which any tree will shed its crown or will be windthrown. The authors go on to describe how time, too, has a role, 
such that storms with a slow build up to their maximum wind speed should cause less windthrow because of the increased time for trees to defoliate and thus decrease wind load interception (Francis \& Gillespie 1993). Likewise, storms with a fast build-up should see more windthrow due to the decreased time to defoliate and thus increased wind load interception (Francis \& Gillespie 1993). Furthermore, the complete dynamic process of windthrow has never been verified in field experiments and the assumption that the maximum wind load produced by the particular event is the key factor in whether damage to trees occurs has never been confirmed (Hale et al. 2010, James et al. 2014).

In summary, the removal of a tree will eliminate the risk associated with that tree but may increase the risk of windthrow of neighboring trees due to changes in the wind regime and exposure (Kane 2008). While tree properties and wind are likely the two largest factors contributing factors to windthrow, the two combined do not explain all observed variation in the windthrow of trees (Kane 2008, Francis \& Gillespie 1993).

\subsection{Conclusion}

While the lLikelihood of fFailure of trees is a hot topic within the arboricultural world, arborists would be wise to remember that many trees will stand throughout the duration of a human lifetime and that a tree once cut may take many years to replace. With regards to UVM this means understanding that the vast majority of trees that stand along ROWs are healthy and do not pose incredible risk by themselves, as it is the combination of the lLikelihood of fFailure, with the Likelihood of Impact, and the Consequences of Failure which ultimately comprise risk. For example, of Kane's (2008) 1259 surveyed trees, only $12.8 \%$ experienced failure or, put another way, $87.2 \%$ of trees survived (Kane 2008). Thus, more varied and targeted approaches to 
tree risk mitigation within UVM may allow for utility foresters to retain financial resources while leaving more trees standing and healthy. 


\section{Chapter 3}

\section{Literature Review of UAS and lidar with application to UVM}

\subsection{Data Acquisition}

In 1971, NASA demonstrated the use of lidar technology to map a portion of the moon surface, and shortly thereafter a number of similar systems were adapted for use on Earth. Primarily spurred on by the need for Digital Elevation Models (DEMs) in forested areas and the technology's ability to estimate tree heights, counts, and even delineate tree crowns, interest in lidar technology began to blossom (Carson et al. 2004; Naesset 2009). Carson et al.'s (2004) paper at the American Society for Photogrammetry and Remote Sensing (ASPRS) annual conference, titled Lidar applications in forestry - An overview, reviewed the applications of lidar to forestry up and unto that point (Carson et al. 2004). In those early years of lidar in forestry, major limitations were unstandardized data collection practices, limited computer processing power, and lack of accurate modelling procedures.

Tiede et al. (2005) developed an early GIS-based workflow for individual tree crown detection and segmentation from lidar data. While the tree detection rates were deemed poor, with only $72 \%$ of dominant trees being detected and only a $51 \%$ overall tree detection rate (Tiede et al. 2005). By 2008, electric utilities had begun exploring both lidar and UAS as tools to improve the management of power lines and the associated vegetation. A case study conducted by Ituen et al. (2008) developed a workflow for analyzing electric lines for risk management purposes. The study suggested that the electrical reliability provided by visual aerial inspection of vegetation along powerlines would be surpassed with use of a combination of lidar and georeferenced imagery (Ituen et al. 2008). Furthermore, the researchers suggested that plant health data may be available in the form of Normalized Difference Vegetation Index (NDVI) from 
spectral analysis of imagery (Ituen et al. 2008). Lastly, the study found that aerial lidar inspection far outpaced traditional visual inspection, completing $50 \mathrm{~km}$ per day of scanning, an equivalent to four days of traditional visual inspection (Ituen et al. 2008).

The following year, Mills et al. (2010) compared lidar and Structure-from-Motion (SfM) photogrammetric techniques for vegetation management of electric ROWs, focusing on the ability to identify the height and position of vegetation relative to the power line. Lidar outperformed SfM in absolute and relative position of vegetation, including both cross track and along track position, and tree height estimation (Mills et al. 2010). The authors concluded that lidar is roughly three times more accurate than SfM (Mills et al., 2010). Through this study, Mills et al. clearly demonstrate the accuracy and potential of aerial lidar technology as applied to UVM.

Frank et al. (2010) demonstrated the capability of high-resolution hyperspectral imagery and lidar used in combination for the mapping, calculation of tree height and conductor clearance, as well as tree species discrimination, on electric transmission lines. The study utilized lidar mounted on a small fixed-wing aircraft, which generated a point cloud of $\sim 28$ points per $\mathrm{m}^{2}$

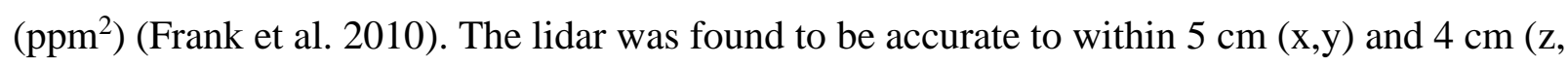
height) (Frank et al. 2010). The study then calculated line clearances between vegetation and conductor and categorized vegetative hazards according to NERC FAC-003 (Frank et al. 2010). Classification of tree species was found to be best with a merged hyperspectral and lidar dataset using a support vector machine (SVM) and resulted in a tree species identification accuracy of 92\% (Frank et al. 2010).

In 2011, a team of researchers from Optech Inc. studied the advantages of aerial lidar for electric line management and included both engineering and vegetation management advantages 
(Ussyshkin \& Theriault 2011). The team's analysis of vegetation included a "vegetation encroachment analysis" and a "tree proximity analysis" (Ussyshkin \& Theriault 2011). The former demonstrated lidar's capability to identify and quantify necessary UVM workload and the latter demonstrated lidar's ability to accurately identify trees that pose a risk to electric lines due to height and location (Ussyshkin \& Theriault 2011).

The following year Ko et al. (2012) published a case study on the mapping of tree genera using aerial discrete return lidar. They predicted tree species from geometric properties of the trees in the lidar point cloud (Ko et al. 2012). While the primary purpose of the study was the identification of tree genera, the study also mapped an electric transmission ROW, identified trees with potential to cause an electrical outage, identified direction of tree lean for trees with sufficient height to fall across electric lines, and located vegetation infringing upon minimum clearance distances, as established by NERC FAC-003 (Ko et al. 2012). In addition, at the 9th International Symposium of Environmental Concerns in Rights-of-Ways Management, Ferguson et al. (2012) outlined how aerial lidar could be utilized for vegetation hazard detection as a method for compliance to NERC FAC-003 while mitigating unintentional environmental impact that may occur during conventional UVM. The authors cite lidar's ability to provide timestamped, auditable, and objective data as the technology's core strength (Ferguson et al. 2012).

Jaakkola et al. (2010) developed a low-cost UAS-based lidar system and tested the feasibility of obtaining accurate tree measurements with the system. The system was the first of its kind in the literature and the study demonstrated its ability to obtain a high-density point cloud for making tree measurements. Field measurements in this study found that the standard deviation of tree heights was $30 \mathrm{~cm}$ (Jaakkola et al. 2010). The standard deviation of tree heights 
according to the UAS-lidar obtained point cloud was $34 \mathrm{~cm}$ (Jaakkola et al. 2010). This publication also provided a glimpse of the change detection capability of such a system, through the successful estimation of biomass change pre- and post-thinning of a scanned stand of Scots pine (Jaakkola et al. 2010).

Wallace et al. (2012) also developed a low-cost UAS-lidar system. Their system was intended for forest inventory and the authors developed a workflow to utilize the new technology, allowing for very high-density point cloud data to be acquired (Wallace et al. 2012). In this initial study, the system was flown at $50 \mathrm{~m}$ AGL and was able to acquire a maximum point cloud density of $63 \mathrm{ppm}^{2}$ (Wallace et al. 2012). Due to the increased point cloud density, decreases in standard deviation of key measurements including tree height, tree location, and crown width were observed (Wallace et al. 2012).

In a subsequent study, Wallace et al. (2014) conducted a study utilizing the UAS-lidar system to again obtain high resolution lidar data, this time for comparing multiple tree detection and delineation algorithms. While the study was primarily seeking to determine the best algorithm by which to detect and delineate trees from within a lidar point cloud; it also assessed the accuracy of each of the algorithms at different point densities and examined the practicality of UAVs as a platform for aerial lidar (Wallace et al. 2014). When using full density lidar point clouds all algorithms detected $90 \%$ of trees, and thus the author concluded that trees within a four-year-old Eucalyptus plantation can be accurately detected and delineated using UAS-based high density lidar (Wallace et al., 2014). The researchers found that point density was more significant than the algorithm used, which was contrary to previous studies on tree detection and delineation from lidar data (Wallace et al. 2014). Due to the more accurate description of tree 
crowns in high density lidar data, the algorithms' measurements of tree crowns more closely matched field measurements and tree locations were more accurate (Wallace et al. 2014).

In an additional follow-up study conducted, Wallace et al. (2016) provided a succinct comparison of two UAS-based, airborne remote sensing technologies: lidar and SfM. Both technologies generate point clouds which can be manipulated to provide information about forest structure, including tree heights, canopy dimensions, biomass, and stem counts. The data acquisition and processing workflows, and forest structure measurement accuracy were assessed for both technologies (Wallace et al. 2016). Point clouds derived from each technology were georeferenced and exported to LAS format and ground points were identified, in both point clouds, and interpolated into a DEM of $10 \mathrm{~cm}$ resolution (Wallace et al. 2016).

Each respective DEM was used for the computation of forest structure metrics: horizontal canopy structure, vertical canopy structure, stem height, stem location, and stem crown area (Wallace et al. 2016). The SfM point cloud had $5652 \mathrm{ppm}^{2}$ and required 24 hours of processing time, compared to lidar point cloud which had $174 \mathrm{ppm}^{2}$ and required 1.5 hours of processing time (Wallace et al. 2016). The two DEM's had almost the same horizontal and vertical accuracy, with a mean difference of $0.09 \mathrm{~m}$, resulting in similar representations of the terrain (Wallace et al. 2016). SfM was less accurate at mapping the terrain under canopy coverage compared to lidar, due to lidar's ability to penetrate the canopy and record multiple returns (Wallace et al. 2016). However, SfM generally failed to provide returns from the middle canopy (Wallace et al. 2016). Of the 136 measured trees on the plot, lidar identified 122, and SfM identified 112 (Wallace et al. 2016). The authors concluded that both technologies are viable remote sensing tools for 3D imaging of forest structure (Wallace et al. 2016). 
Lidar has quickly become one of the premier aerial data types, as it can provide unprecedented detail and accuracy. UAS-based lidar has the advantage of being flown closer to the ground compared to traditional aerial platforms, allowing for more returns per unit area, which allows for the creation of very high-resolution point cloud datasets (Wallace et al. 2012). While these datasets require major data processing resources, the information obtained from them can be particularly useful. Given that aerial lidar from larger, manned aircraft has been used to map and monitor transmission electric ROWs vegetation and line sag conditions; and that UAS-based lidar has been utilized for forest inventories, the application of UAS-based lidar to mapping and monitoring the distribution electric grid appears to be a natural fit (Frank et al. 2010, Kim \& Sohn 2013, Ussyshkin \& Theriault 2011, Wallace et al. 2012).

\subsection{Data Processing}

\section{Digital Elevation Model (DEM) Interpolation}

Foresters were some of the first to suggest utilizing lidar for DEM creation due to its ability to penetrate vegetation cover (Carson et al. 2004). For examples of early examples of lidar derived DEM creation see Carson et al. (2004) or Maclean \& Krabill (1986). For an example of a more modern method of DEM generation, see Zhang et al. (2003). DEMs, and thus, DEM interpolation, are extremely important to lidar-based Utility Vegetation Management (UVM), as the DEM will provide the ground level for all height measurements, including both trees and electrical infrastructure.

\section{Point Cloud Classification}

Classification of a lidar point cloud, where each return is given a label based on the surface that returned the pulse (vegetation, building, ground, etc.), is time consuming and 
expensive, particularly if it is completed manually (Brunker 2016). Yet, a higher level of detail in point cloud classification allows for more accurate individual tree detection and segmentation, and tree-to-conductor proximity analysis (Brunker 2016). Due to the volume of data necessary to manage electrical infrastructure with lidar, automated classification of electrical ROW scenes is necessary for effective management (Kim \& Sohn 2013).

To this end, Kim and Sohn explored the potential of using Random Forest, a supervised learning classifier, to classify five key objects (vegetation, pylons, wire, buildings, and low objects) from aerial-lidar point clouds acquired of a power line corridor in Sacramento, CA. The researchers compared a grid-based classification scheme to a point-based classification scheme, then distinguished which of 21 features associated with each point were most useful in the classification (Kim \& Sohn 2012).

Kim and Sohn's results showed that Random Forests more accurately classified power line corridor scenes after being trained with balanced training data $(97.95 \%)$ compared to unbalanced training data which resulted in an accuracy of $96.62 \%$. Of the 21 point features assessed for classification relevancy, 12 were ultimately selected (Kim \& Sohn 2012). Using balanced training data, and the 12 key classification point features, a sample -weighted accuracy of $91.04 \%$ and a class weighted accuracy of $90.07 \%$ was achieved (Kim \& Sohn 2012). The point-based classifier was more accurate than the grid-based classifier by $4.86 \%$ in sampleweighted accuracy and $5.74 \%$ in class-weighted accuracy (Kim \& Sohn 2012). Despite the relative accuracy of their methods, research aiming to improve automated classification should continue, as $\sim 90 \%$ classification accuracy across the entire electrical grid would result in many errors and hours of data clean-up (Kim \& Sohn 2012). 


\section{Individual Tree Crown (ITC) detection and segmentation}

Individual Tree Crown (ITC) detection and segmentation has long been a goal of remote sensing, in fact many modern CHM-based methods trace their roots back to methods developed for aerial imagery. For an overview of ITC detection and segmentation from aerial imagery, see Brandtberg et al. 2003. The ITC methods which lidar and aerial imagery share include: local maxima (LM),region growing (RG), watershed (WS), valley-following (VF), scale-space theory (SS), template matching (TM), Markov random fields (MRFs), and marked point processes (MPP) (Larsen et al. 2011; Mohan et al. 2017).

In 2012, Li et al. argued that converting from point cloud to raster data type is not ideal for individual tree detection since the interpolated raster surface, typically a CHM, has many potential sources of error ( $\mathrm{Li}$ et al. 2012). Li et al. utilized an individual tree detection and segmentation algorithm which detects and segments trees directly from the point cloud (Li et al. 2012).

More recently, Hamraz et al. (2016) demonstrated a robust method for tree segmentation directly from the point cloud for deciduous forests. The non-parametric approach identified $94 \%$ of dominant and co-dominant trees and obtained a false detection rate of 13\%; overall accuracy was $77 \%$ (Hamraz et al. 2016). The approach works through an iterative process of global maximum identification, generation of vertical profiles radiating from global maximum, identification of the last point before "between-tree gap" and/or the local minimum from each vertical profile. Then a convex hull is created from points identified in previous step, and all points within the delineated convex hull boundary are segmented into a unique tree crown (Hamraz et al. 2016). The process is then repeated on the remaining points in the point cloud (Hamraz et al. 2016). 


\section{Derivation of tree biophysical properties}

Once trees have been detected and segmented from the point cloud, biophysical information about the trees can then be inferred based upon the distribution of points assigned to that tree. Information of particular interest to UVM includes tree species and/or genera identification for estimated growth and failure rates, tree height and location, as well as line height and location, to determine if the tree could come into contact with electric lines. Tree species/genera identification from a lidar point cloud is also well documented within the literature, see Holmgren et al. (2008), Ko et al. (2012), Korpela et al. (2007), Korpela et al. (2010), or Persson \& Holmgren (2004).

obtaining tree height from lidar data is aso well documented in the literature. For examples of this process, see Persson \& Holmgren (2004), Popescu \& Wynne (2004), or Suárez et al. (2005). Yet, using tree height to determine NERC FAC-003 classification is relatively uncommon in the literature, with only two examples, Ko et al. (2012) and Ferguson et al. (2012), explicitly describing this process. While identifying tree height by itself is useful for NERC FAC-003 vegetation threats, it is lacking a few critical pieces of information: the electrical conductor height and the difference in elevations between the tree and the conductor. Additionally, direction of tree lean would aid in further refining tree risk assessment tree-caused damage of utility lines.

Few studies have attempted to assess tree lean from aerial lidar data. Ko et al. (2012) used a vertically sliding voxel to identify the centroid of a given voxel, then connected the lowest centroid to the highest via a straight line, allowing for an assessment of estimated tree lean (Ko et al. 2012). Hamraz et al. (2016) used a similarly assessed tree lean angle as an input for position agreement test, where field surveyed tree bases were linked to lidar obtained treetops (Hamraz et 
al. 2016). Importantly, the accuracy of tree lean approximations from lidar has not been evaluated in the literature. 


\section{Chapter 4}

\section{Assessment of UAS-lidar system for UVM}

\subsection{Introduction}

The Utility Vegetation Management (UVM) of distribution electrical rights-of-ways (ROWS) face several unique and significant challenges. Among these are the miles of rugged and inaccessible ROWs, many lined with trees which, should they fall, possess the height necessary to contact the electrical conductors, potentially damaging electrical infrastructure or causing an electrical fault resulting in a power outage. In 2017, the American Society of Civil Engineers (ASCE) has released their Infrastructure Report Card which details the current infrastructure conditions and needs within the US. The United States scored a "D+" under the "Energy" category, due to aging infrastructure and a lack of resilience in the face of severe weather events. A list of recommendations provided by the ASCE included, "Promote usage of remote sensing and inspection technologies to lower the cost of energy system monitoring; focus operation and maintenance spending on highest-risk system components" (Energy 2017).

among emerging remote sensing technologies, lidar and Unmanned Aerial Systems (UAS) are particularly promising for distribution vegetation management, as they may provide a faster, more accurate, and ultimately, more cost-effective method for inspection compared to traditional UVM, where inspection is done visually by foot patrol, potentially over rugged terrain. Due to this rapid and accurate identification of vegetation threats, individual trees and problematic areas may be targeted for more prescriptive management (Miller et al. 2015). Furthermore, lidar has been utilized to aid in the UVM of larger transmission electric lines (Ituen et al. 2008, Mills et al. 2010, Frank et al. 2010, Ussyshkin \& Theriault 2011, and Ko et al. 2012). Additionally, Unmanned Aerial Systems (UAS) have been combined with lidar to develop 
platforms for forest inventories (Jaakkola et al. 2010, Wallace et al. 2012, Wallace et al. 2014, and Wallace et al. 2016). Given these separate but related research directions, the combined use of UAS and lidar for distribution electric UVM seems to be a logical extension of the application of these new technologies.

This study looks to: (1) examine how Digital Elevation Model (DEM) interpolation methods affect individual tree crown detection and segmentation routines and tree risk analysis from high-density lidar, (2) evaluate the effect of point cloud decimation on individual tree crown detection and segmentation routines and tree risk analysis, (3) develop a tree base location approximation method for the identification of NERC Category $2 \& 3$ vegetation risks, and (4) develop a methodology to estimate the Likelihood of Impact of a NERC Category 2 \& 3, "fallin" vegetation risks on the electric lines.

Additionally, while NERC FAC-003 regulations do not apply to distribution electrical infrastructure and its maintenance, it does provide a useful classification of vegetation threats. In particular, we focus on Category $2 \& 3$ threats, which are "fall-in" threats. These categories of threats are differentiated by their base locations, on-ROW and off-ROW, respectively. We emphasize in using this classification scheme, the authors are not suggesting that NERC FAC003 regulations be applied to the distribution grid.

\subsection{Methodology}

\section{Study Area}

The study area comprises $1.6 \mathrm{~km}$ (1 consisted of one linear mile) of a distribution electric circuit and its associated ROW, located in North central West Virginia. The study area was selected because within a small area it contains many elements representative of the region in terms of both the electrical infrastructure and ROW situations, most notably variable topography 
and backgrounds, such as a creek crossing, and in places lack of roadside access. The circuit possesses both three-phase $12.5 \mathrm{KV}$ and single phase $7.2 \mathrm{KV}$ distribution electric configurations. The $12.5 \mathrm{KV}$ line leaves the substation located at the North end of the study area and runs south along the road, slightly uphill of a creek that is buffered by tall and dense vegetation. Two 7.2 $\mathrm{KV}$ lines split off of the $12.5 \mathrm{KV}$ line near the center of the study area. One runs southwest across the creek then uphill towards a larger transmission ROW and the other runs east, crosses the road and several lawns, before heading uphill and leaving the study area. In addition to variable elevation, the area features "open" areas, such as lawns, where tree canopies grow differently due to the lack of competition for light; and closed -canopy, forested areas, where canopy growth is constrained by competition for light from other trees. Also, some segments of ROW are forested on one side and open on the other, while other segments are forested or open on both sides.

Our research team utilized a Geodetics Inc. UAS-lidar mapping system based on a DJI Matrice 600 platform equipped with the Geo-MMS Velodyne VLP-16 lidar sensor. The system was flown at $70 \mathrm{~m}$ Above Ground Level (AGL), at a speed of $10 \mathrm{~m} / \mathrm{sec}$. Two separate flight lines were made down the center of the each of the ROWs to cover the study area. Figure 1 shows the study area and the flight plan for the second of the two flight lines. For a visualization of our data 
processing workflow, see Figure 2.



Figure 1: Study Area and Flight Line 2 


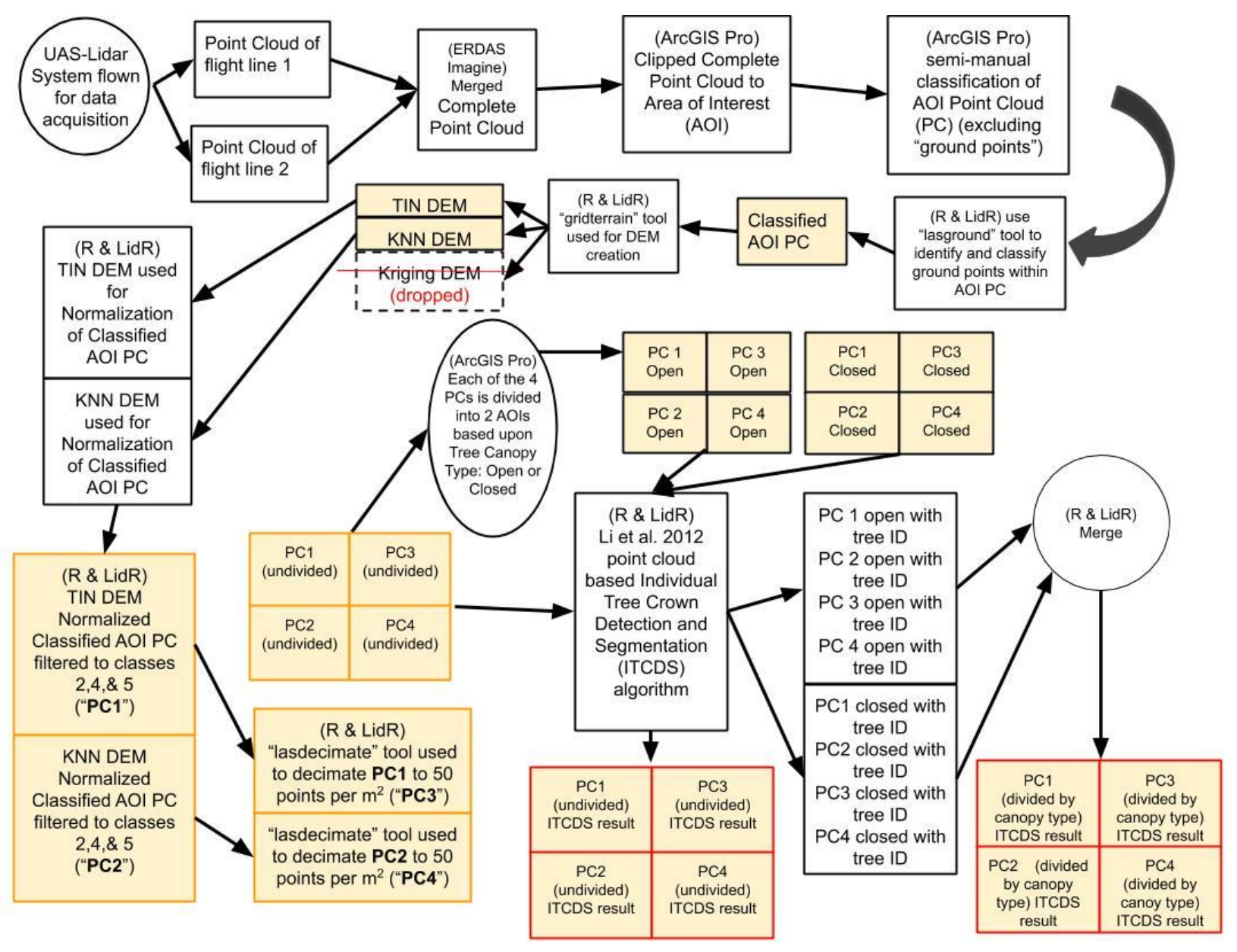

Figure 2: Lidar processing workflow

The aerial acquisition yielded two point clouds, one from each flight line. The point clouds were merged in Hexagon's ERDAS Imagine 2016 and clipped to our area of interest (AOI), resulting in a final point cloud of $30,059,825$ points with a nominal point spacing of $0.0515 \mathrm{~m}$ per point, equivalent to a point density of $377 \mathrm{ppm}^{2}$. A digital surface model (DSM) with $0.5 \mathrm{~m}$ resolution of the study area is shown in Figure 3. The scans, while successful in capturing the poles, vegetation, buildings, and other structures; did not capture the conductor wires. Furthermore, the conductor wires may have been filtered out as noise by Geodetics' preprocessing software before we received the point clouds. Future investigation into whether conductor lines were missed or simply filtered out of the point cloud would be useful. 


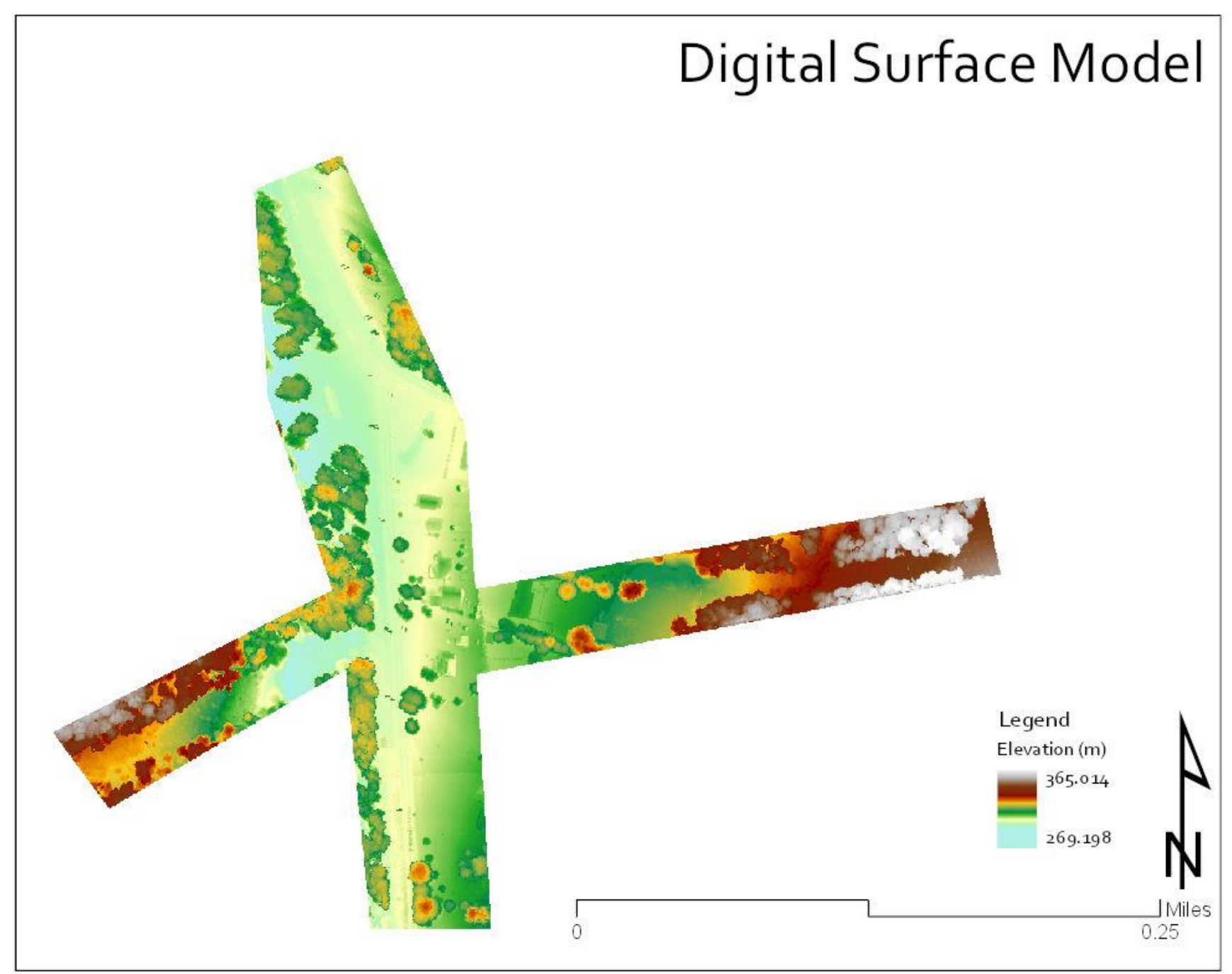

Figure 3: Digital Surface Model (DSM) 


\section{Pre-Processing the point cloud}

After the pre-processing steps of acquisition, merging, and clipping to the AOI, the point cloud was classified using R 3.4.1, the R package, “lidR” 2.0.1 (Roussel et al. 2020), and ESRI's ArcGIS PRO 2.0. First, we utilized lidR's implementation of the Progressive Morphological Filter, developed from the algorithm of Zhang et al. (2003), to identify and modify the classification of "ground" points. The point cloud was then imported into ArcGIS PRO, where "Classify by Height" was used on the remaining unclassified points to make approximate classes. Objects less than or equal to $2 \mathrm{~m}$ were labeled "low vegetation", objects greater than $2 \mathrm{~m}$ and less than $6 \mathrm{~m}$ were labeled "medium vegetation", and objects greater than or equal to $6 \mathrm{~m}$ were labeled "high vegetation". The remaining classes of "noise", "building", "electric wire", "electric pole", and "road surface", were classified manually. A rasterized classification map is show in Figure 4 and classified 3D point cloud map shown in Figure 5.

The study area AOI was split into two smaller AOIs based on dominant vegetation canopy type: a closed canopy AOI dominated by forest, and an open canopy AOI characterized by lawns with occasional open-grown trees (Figure 6). For simplicity, the sub AOIs were delineated manually; in an operational workflow this step could potentially be automated by utilizing a measure of tree density.

The digital elevation models (DEMs) were generated on a $0.5 \mathrm{~m}$ grid using lidR's "grid_terrain" function (Roussel et al. 2020). The tool outputs a rasterized surface interpolated from the points classified as "ground" in the point cloud. The tool offers multiple methods for interpolation, including triangulated irregular network (TIN), inverse distance weighted k-nearest neighbor (KNN), and kriging. See Figures 7, 8, and 9 for DEMs generated using TIN, KNN, and kriging, respectively. Additionally, to more closely inspect differences between the DEMs, 
ArcGIS Pro's "Raster Calculator" was used to subtract one DEM from another to visualize where the differences in interpolation occurred, see Figure 10.

DEM accuracy is crucial to this study because the DEM affects how the point cloud is normalized, and thus, has a role in the measurement of the height above the ground. This study utilizes four measurements which rely upon the DEM, either directly or indirectly: tree height, tree base elevation, line height, and the elevation at line impact location. Based on these measures, the kriging DEM was dropped from the study and not used to normalize the point cloud, as it has numerous, extreme interpolation and edge artifacts. While these types of errors occur in each of the DEMs, the kriging DEM displayed the most dramatic examples, see the subset images in Figure 9.

Next, copies of the TIN DEM and the KNN DEM normalized point clouds were decimated to $50 \mathrm{ppm}^{2}$ using lidR's "lasfilterdecimate" function. The resulting four point clouds, TIN normalized full density (PC1), KNN normalized full density (PC2), TIN normalized decimated (PC3), and KNN normalized (PC4). Then, each was filtered by class: "ground", "medium vegetation", and "high vegetation". In early attempts, "low vegetation" was also included, but its inclusion caused problems in the individual tree crown detection and segmentation routines. Therefore, it was removed from the point clouds before individual tree detection and segmentation routines.

Canopy height models (CHMs) were generated for each point cloud. The CHMs were not used in the tree detection and segmentation routines utilized here, since the method from Li et al. (2012) works directly within the point cloud. These CHMs were generated to visualize the effects of DEM interpolation methods on normalization of the point cloud, as well as to visualize the effects of decimation. 
The CHMs were produced using lidR's “grid_canopy” function (Roussel et al. 2020). This function outputs an interpolated raster surface of the uppermost points in the point cloud. All CHMs are $0.5 \mathrm{~m}$ resolution and were generated using the pit-free algorithm from Khosravipour et al. (2014), see Figures: 11, 12, 13, and 14. Additionally, to visualize differences in the CHMs ArcGIS PRO's "Raster Calculator" was used to subtract one CHM from the other to highlight the differences between the two surfaces, see Figure 15. 


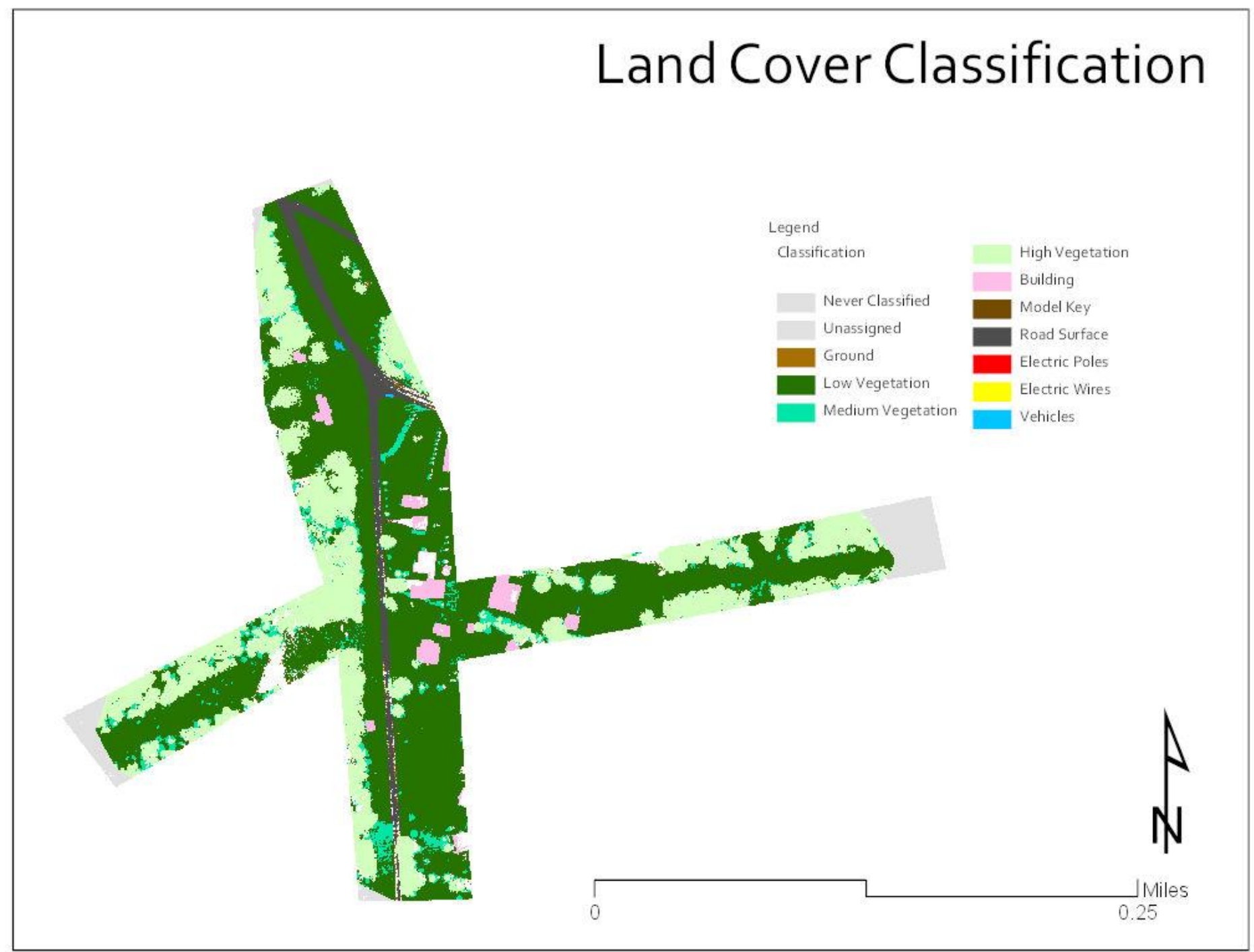

Figure 4: Land Cover Classification 


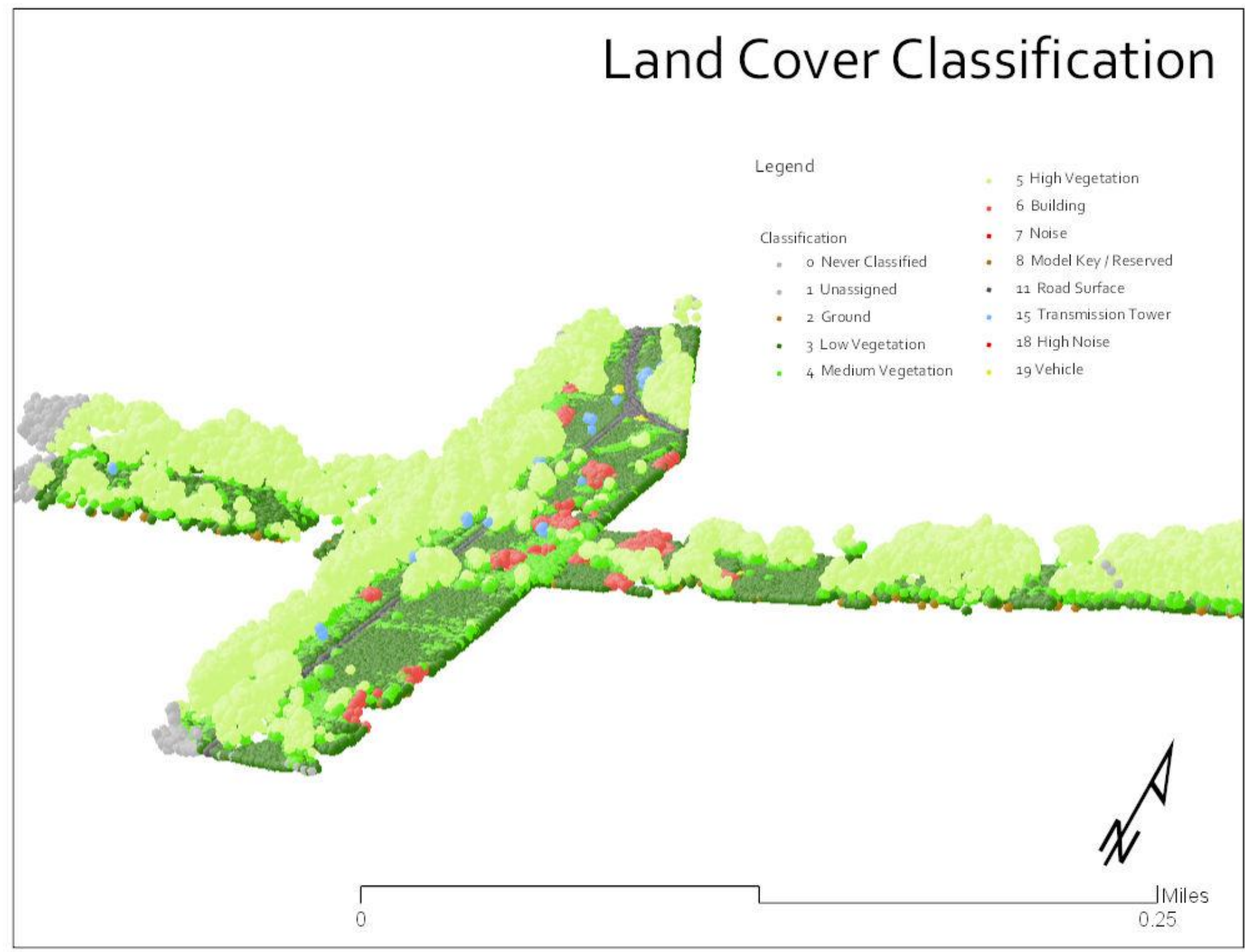

Figure 5: Perspective View of the Classified Point Cloud 


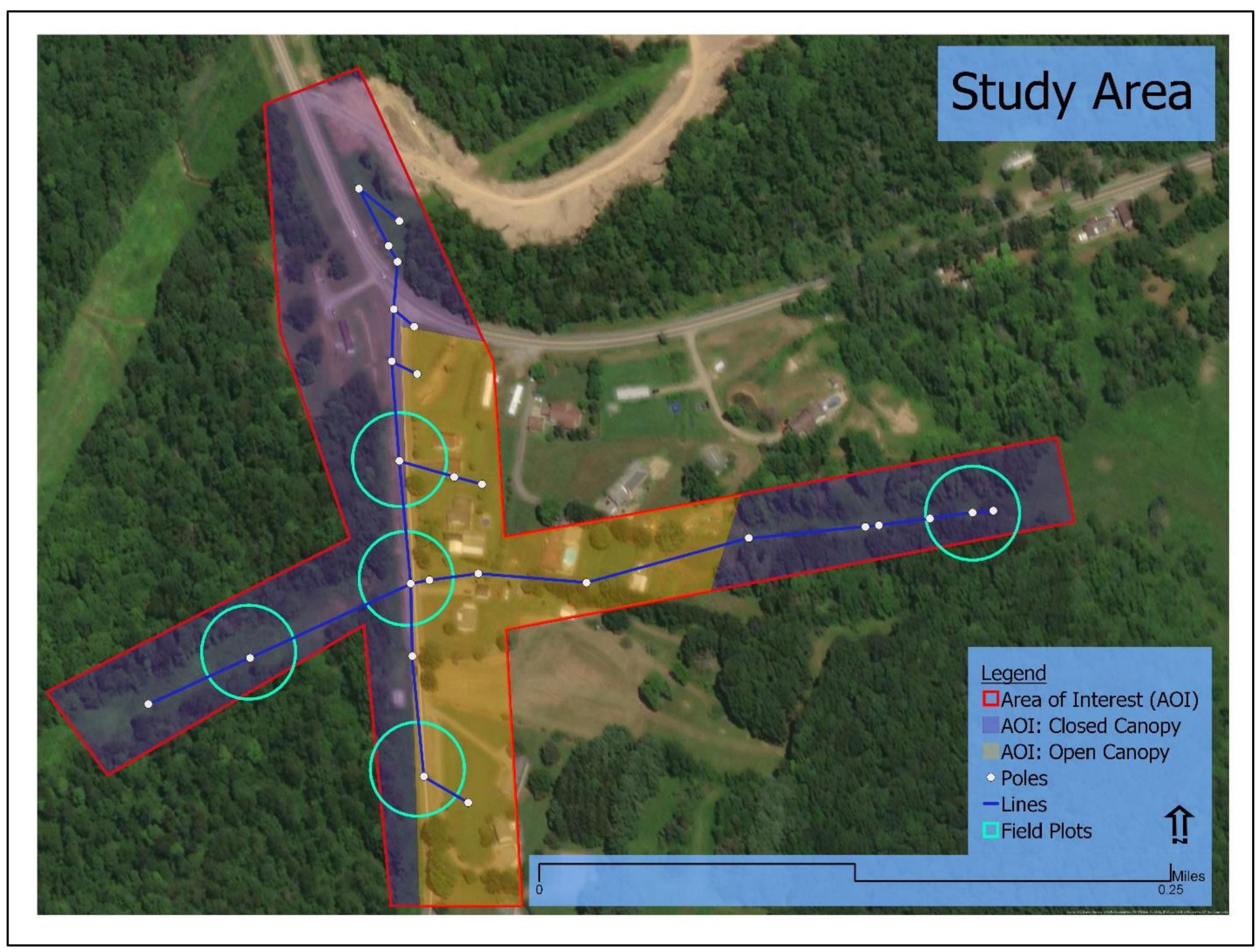

Figure 6: Areas of Interest with Field plots 


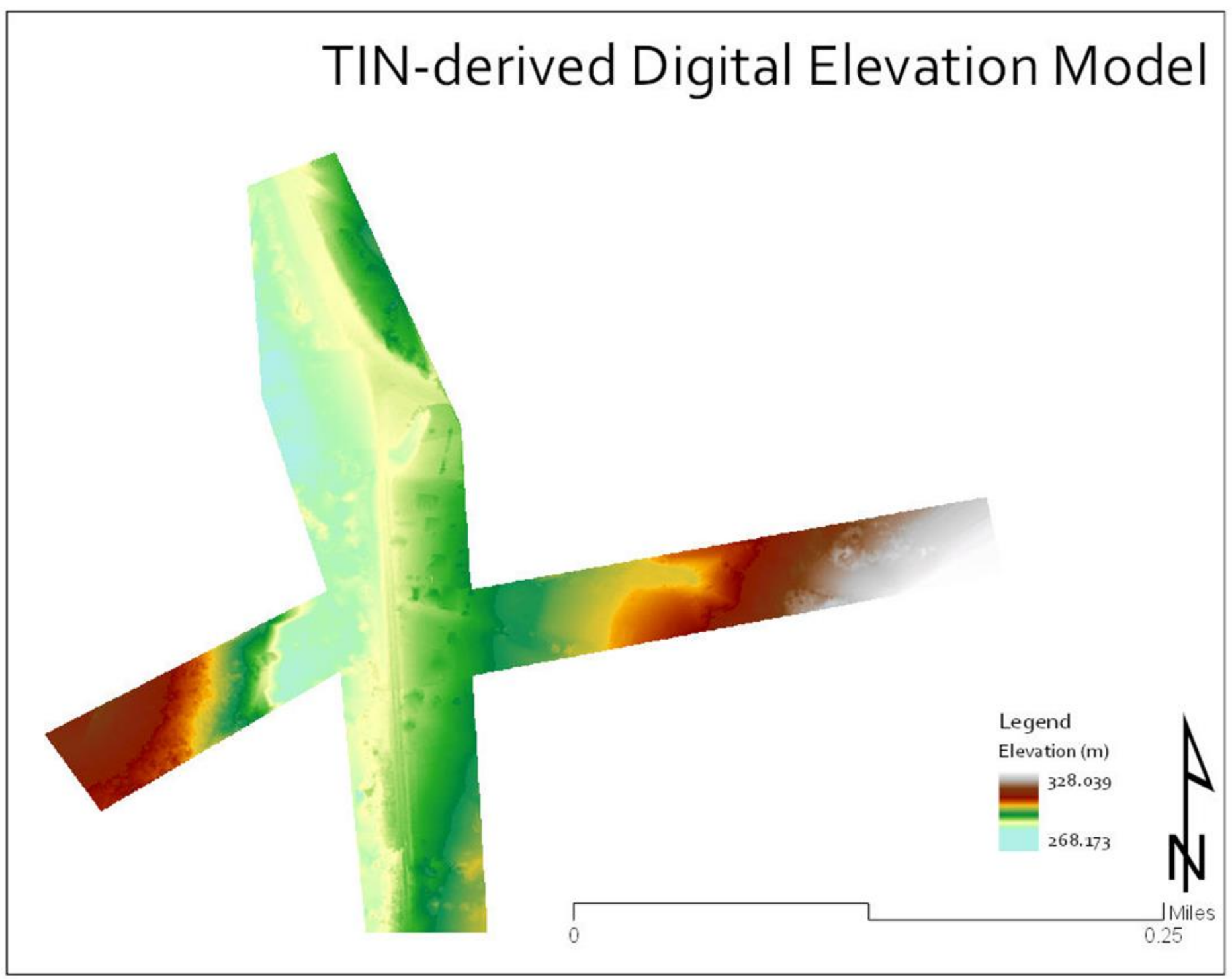

Figure 7: Triangulated Irregular Network (TIN) DEM 


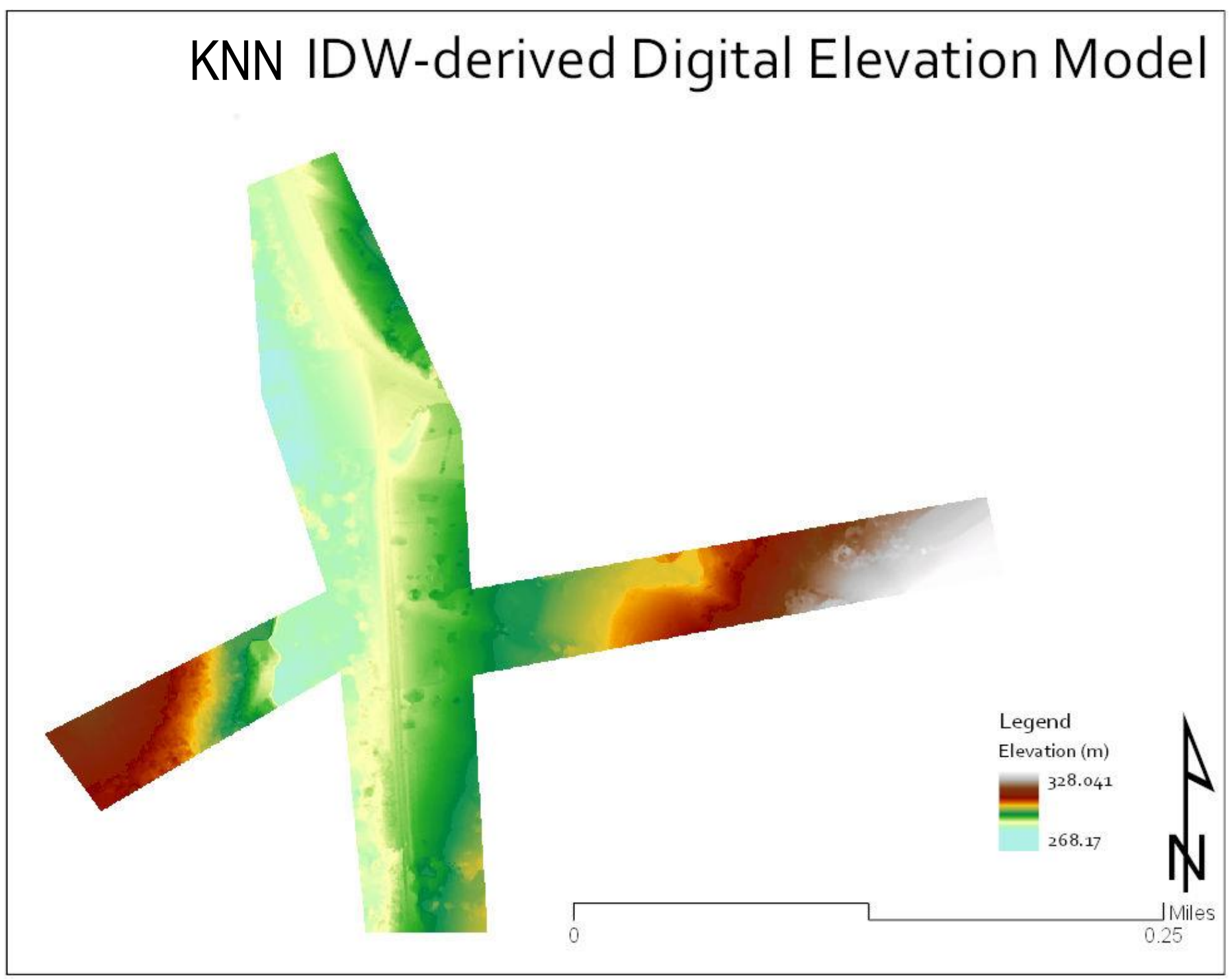

Figure 8: Inverse Distance Weighted K-nearest neighbor DEM 


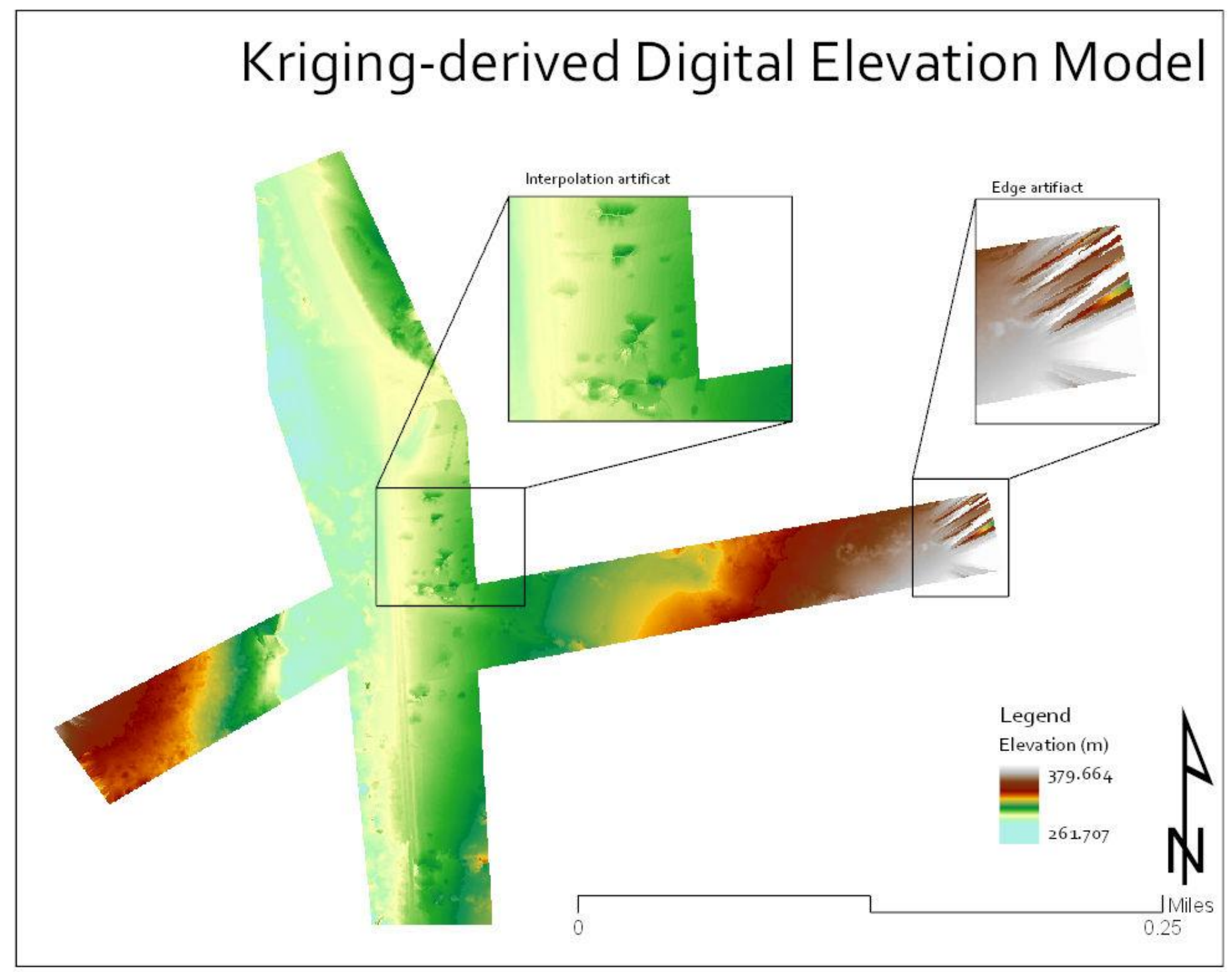

Figure 9: Kriging DEM 


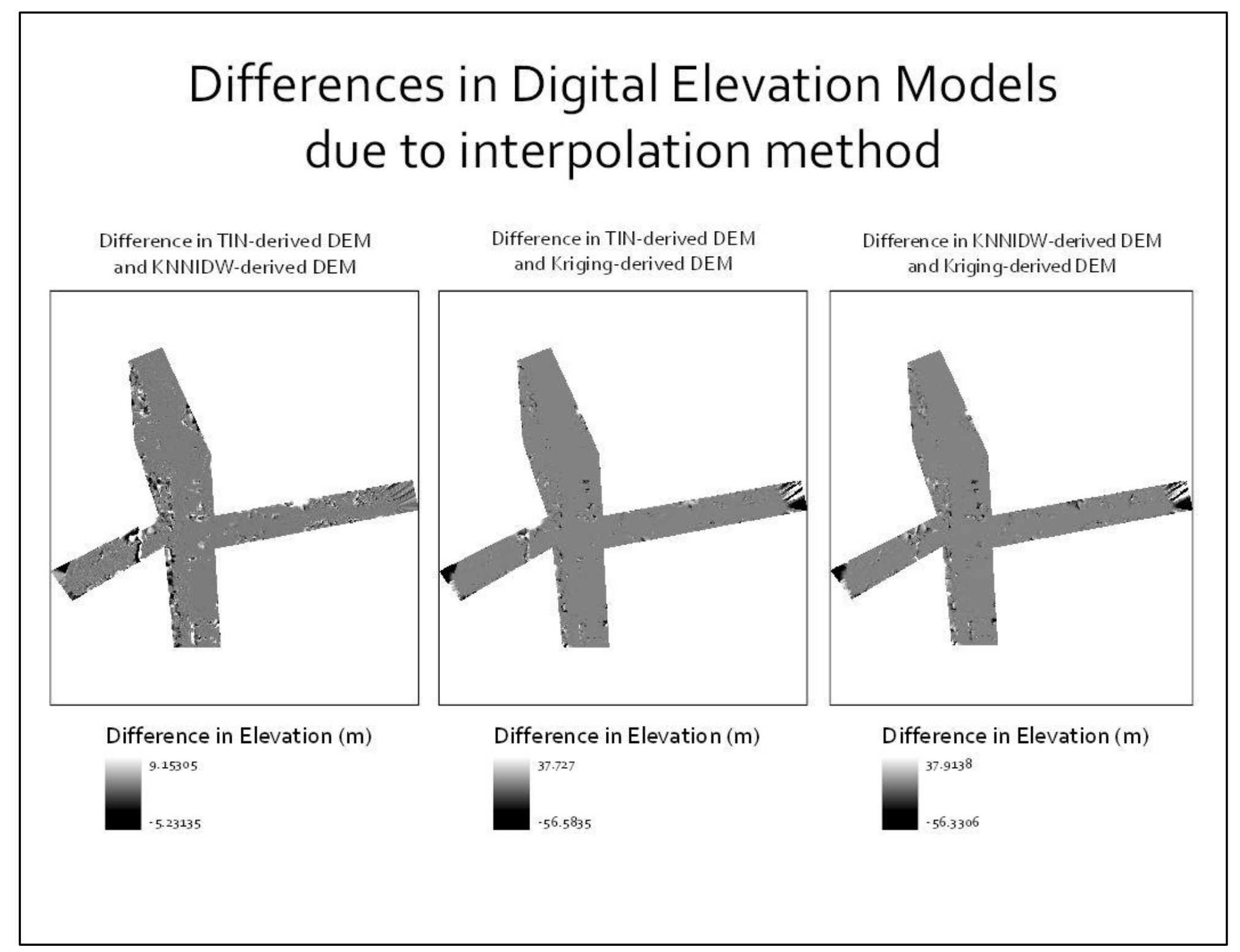

Figure 10: Differences between the DEMs based on interpolation method 


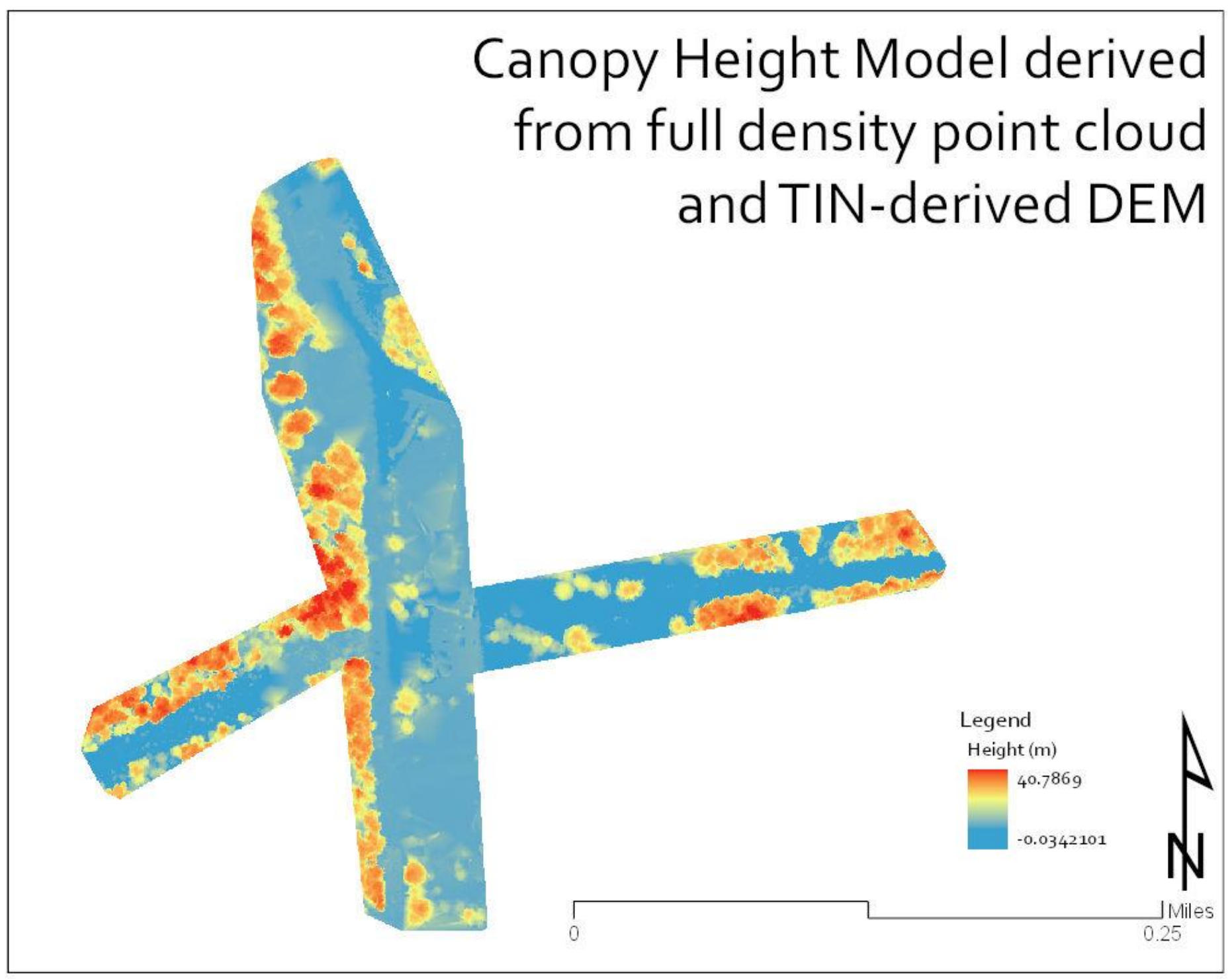

Figure 11: Canopy Height Model (CHM) for TIN DEM normalized, full density point cloud (PC1) 




Figure 12: Canopy Height Model (CHM) for the TIN DEM normalized, decimated point cloud (PC 3) 




Figure 13: Canopy Height Model (CHM) for the KNN DEM normalized, full density point cloud (PC2) 


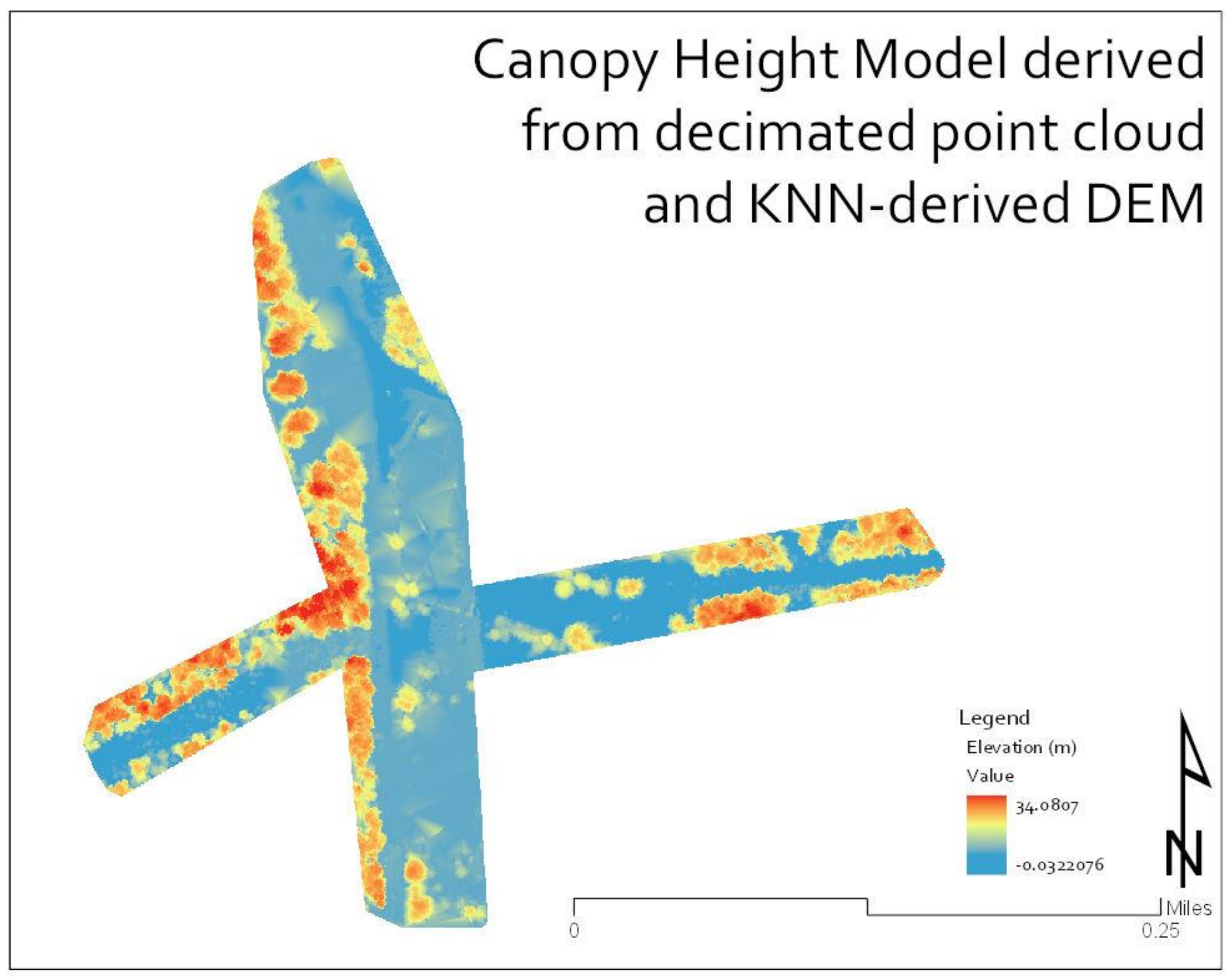

Figure 14: Canopy Height Model (CHM) for the KNN DEM normalized, decimated point cloud (PC4) 


\section{Differences in Canopy Height Models}
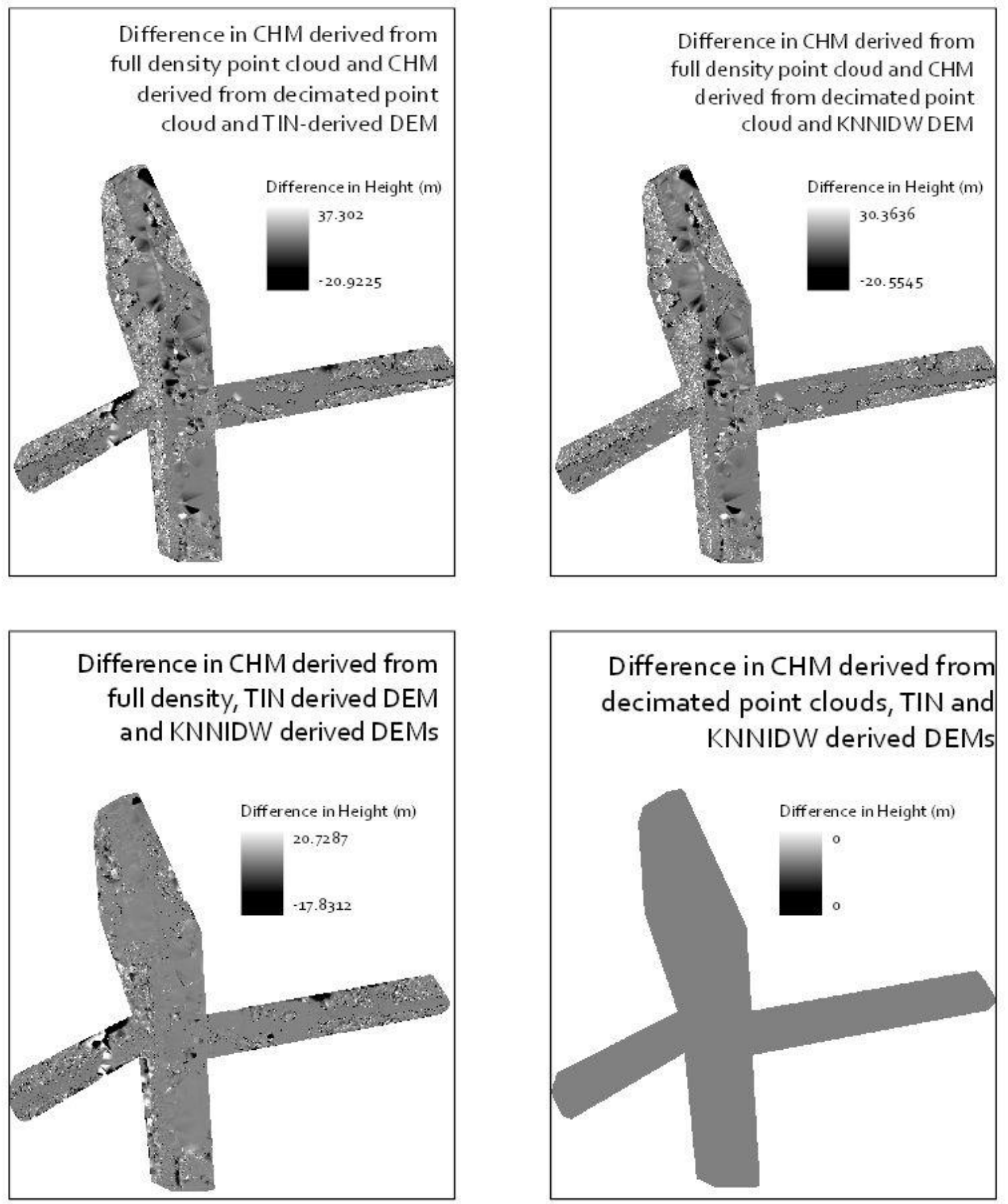

Figure 15: Comparisons of Canopy Height Model differences. 
The full density CHMs generally showed smaller artifacts than those of the decimated CHMs. The differences between CHMs derived from full density point clouds were minor and generally concentrated on the western leg of the scan and north side of the right-of-way. There were essentially no differences between the CHMs derived from decimated point clouds.

We utilized the two smaller vegetation canopy type AOIs (Figure 6) to divide the point cloud into two areas, based on the dominant canopy. This resulted in a total of 12 point clouds, PC1 - 4 (the entire study area), and four dominantly "open canopy" point clouds and four dominantly "closed canopy" point clouds. We hypothesized that the point clouds from the smaller AOIs would produce better results than the point clouds from the entire study area, since with more uniform land cover, the parameters for Li et al.'s (2012) algorithm can be tailored to the characteristic tree spacing.

\section{Individual Tree Crown Detection and Segmentation}

The implementation of Li et al.'s (2012) individual tree detection and segmentation in the lidR package (Roussel et al. 2020) was utilized for individual tree crown detection and segmentation from each the normalized point clouds. This method works directly on each of the points in the point cloud and does not require interpolated canopy surfaces. Thus, its author concludes that the method simplifies the coding process and potentially decreases the sources of error (Li et al. 2012).

Pirotti et al. (2017) found that Li et al.'s (2012) method performed the best overall of their examined individual tree detection and segmentation methods. Its performance in terms of False positives (measured by the metric, precision) was comparatively poor, though it made up for this by having low false negatives, as measured by Recall. Given the emphasis on safety and reliability of electrical power and the hazardous situation potentially presented by NERC 
category $2 \& 3$ vegetation threats, these type II errors should be minimized. Yet, Pirotti et al. (2017) noted that the major drawback to this method was the processing time required to detect and segment trees from within the point cloud. We took these facts into consideration when selecting Li et al.’s (2012) point cloud based individual tree detection and segmentation algorithm.

Li et al.'s (2012) algorithm utilizes six parameters; two dynamic thresholds, a search radius, the decision height, minimum tree height, and maximum crown radius, to detect and provide a "treeID" to each point in the point cloud ( $\mathrm{Li}$ et al. 2012). Points above the decision height are examined using the second dynamic threshold, while points below the decision height are examined using the first dynamic threshold. The dynamic thresholds are values which dictate the point spacing used to determine whether a point belongs within a particular "treeID" (Li et al. 2012). While the minimum tree height aids in controlling over-segmentation, particularly of lower limbs into separate trees, the maximum crown radius aids in controlling under segmentation by providing a limit on the size of tree crowns (Li et al. 2012).

After trial and error, separate parameters were determined to best detect and segment trees within each group of point clouds: undivided point clouds, closed canopy point clouds, and open canopy point clouds. Then, using the parameters determined for each group, the algorithm was run for each point cloud, such that all of the undivided point clouds used the same set of parameters, then all of the closed canopy point clouds used their set of unique parameters, and finally all the open area point clouds used their set of unique parameters.

The results of Li et al.'s detection and segmentation routine for the closed canopy and open canopy point clouds were then merged to produce a single map of the entire study area. We refer to this group of models as our "divided canopy" models. This is In contrast to those which 
used a single undivided point cloud, which we refer to as "single canopy" models. For examples of individual tree detection and segmentation issues see, Figure 16. For a selection of our individual tree detection and segmentation results, see: Figures: 17, 18, 19, and 20.



Figure 16: Three D Perspective View of a Selected Portion of the Individual Tree Detection and Segmentation 


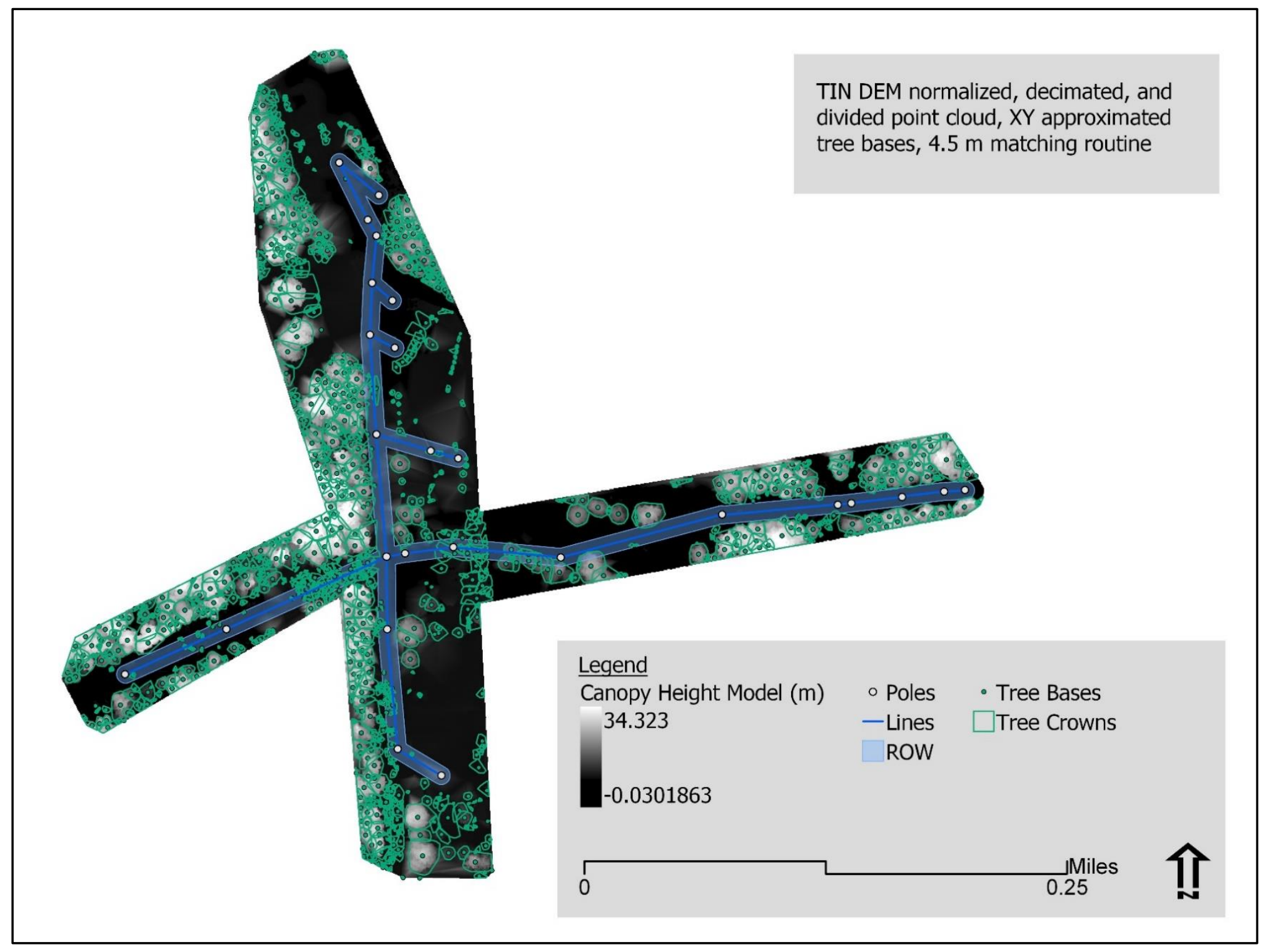

Figure 17:Individual Tree Crown Detection and Segmentation Result, example 1 


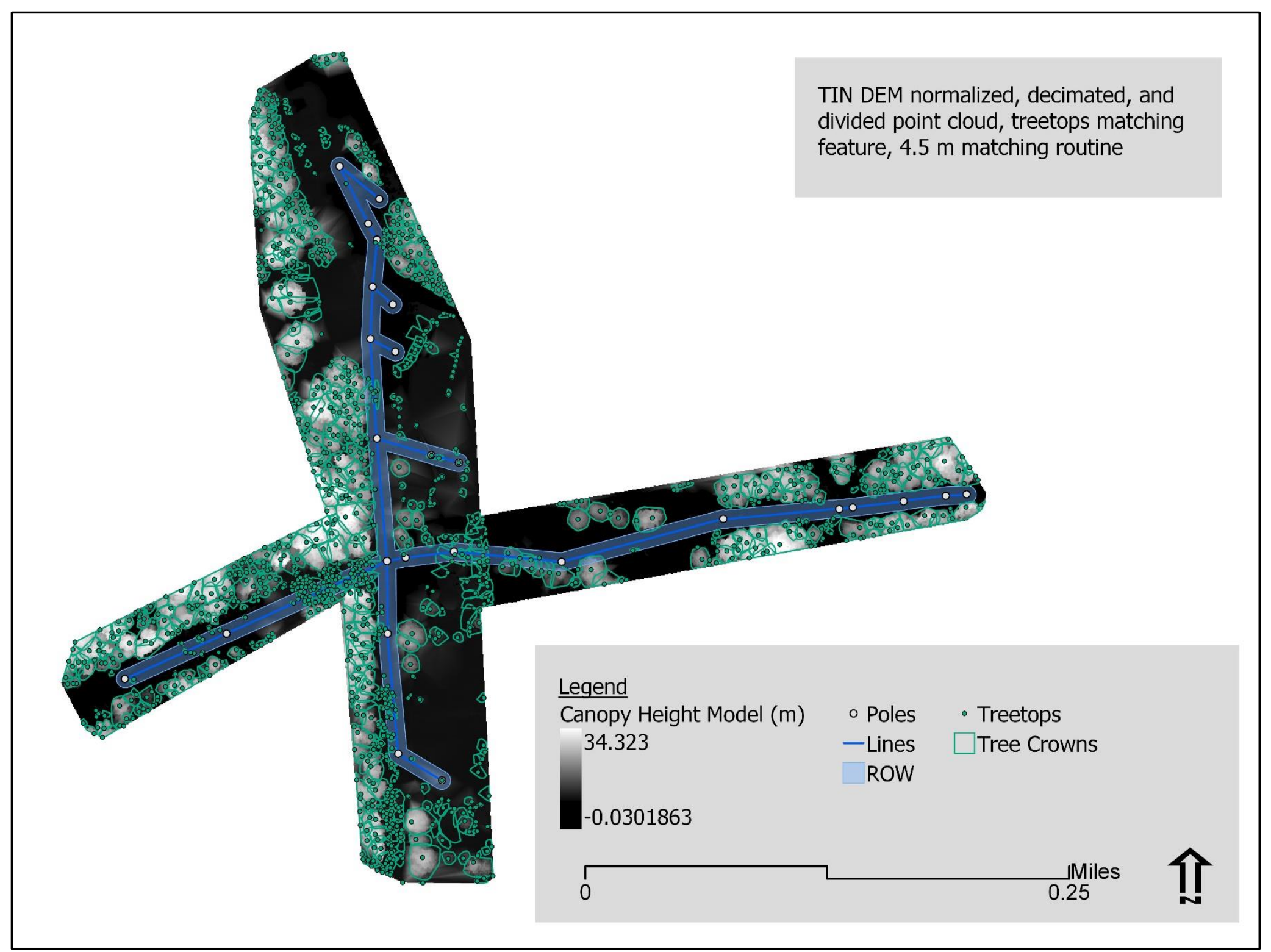

Figure 18: Individual Tree Detection and Segmentation Result, example 2 


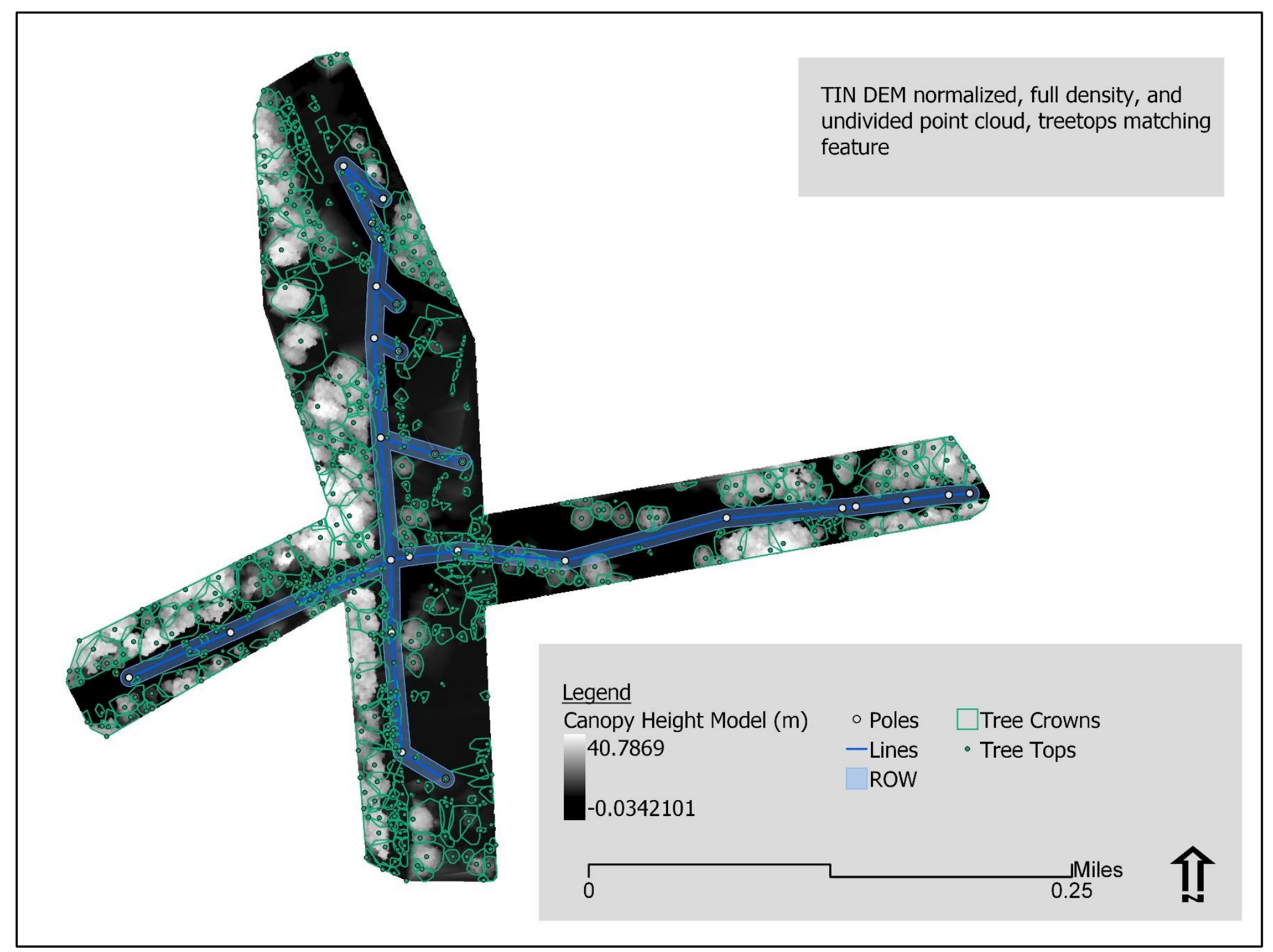

Figure 19: Individual Tree Detection and Segmentation Result, example 3 




Figure 20: Individual Tree Detection and Segmentation Result, example 


\section{Pole Detection}

Li et al.'s (2012) individual tree detection and segmentation algorithm was used to automatically detect and segment electrical pole structures. The workflow for this process mirrors the earlier individual tree detection and segmentation, just simplified such that a single DEM and point cloud were used. See Figure 21 for a visualization of this workflow. Also, rather than filtering to the vegetation classes, the classes "never classified", "unclassified", and "ground" were utilized. This method quickly identified all the pole's in the AOI and provided each with a unique identifier.

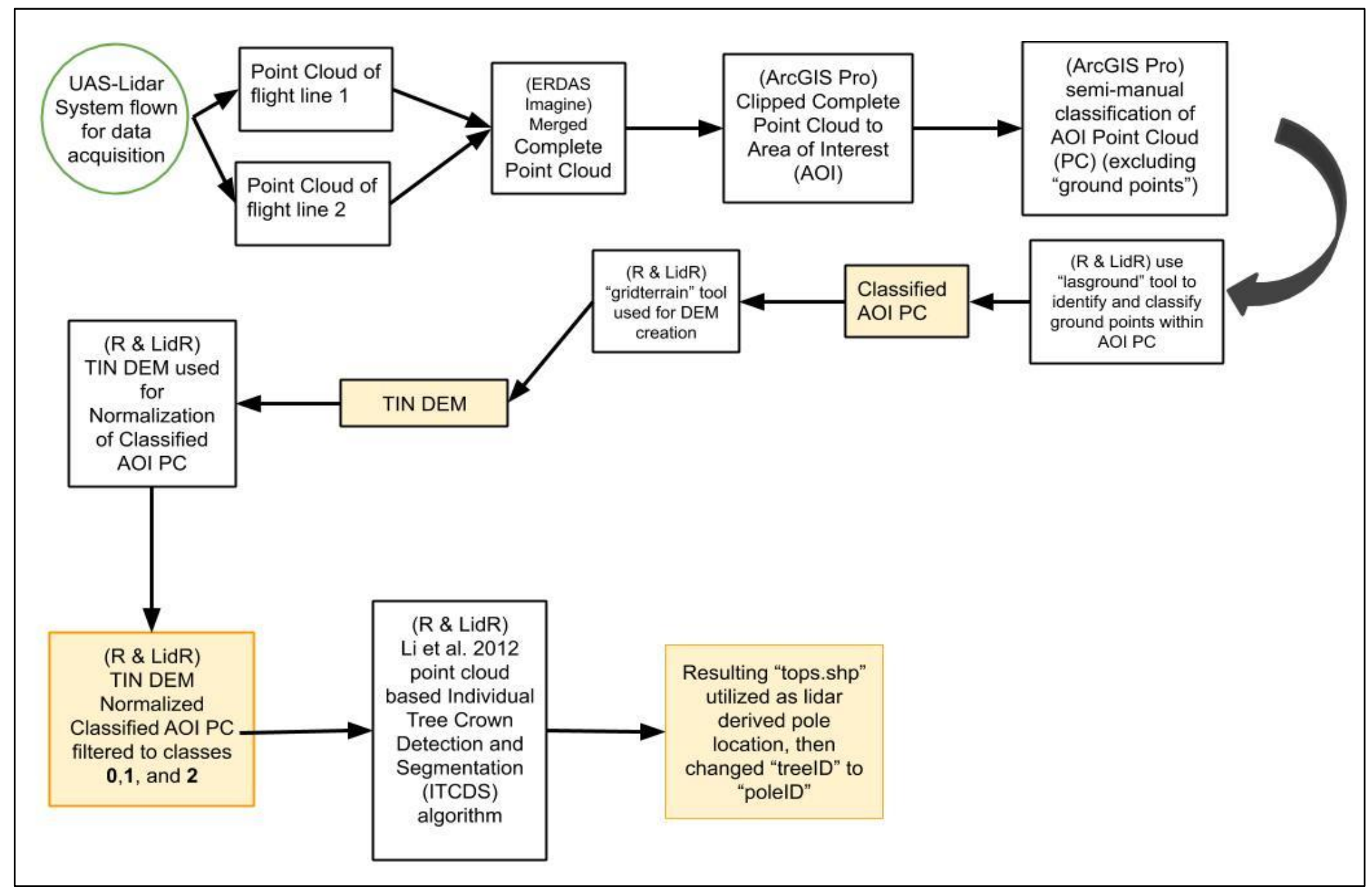

Figure 21: Pole Detection and Segmentation 


\section{Matching Routines for the Accuracy Assessment}

Tree matching routines are necessary to compare the trees identified through individual tree crown detection and segmentation routines and lidar data with field -measured trees. For examples of matching routines in the literature see Larsen et al. 2011, Esyn et al. 2015, Vaughkonen et al. 2012, Kaartinen et al. 2012, Pirotti et al. 2017, and Pirotti 2010.

This study utilized two rules for the matching algorithm. The first was that lidar -derived tree height had to be within a certain percentage of the field measured tree height. A threshold of +/- 15\% was chosen based upon the Nikon Forestry Pro handheld laser's precision, which is claimed to be $+/-15 \%$ of total measured tree height. We also evaluated accuracy using Esyn et al.'s (2015) 10\% threshold of the tree height.

The second matching requirement was based upon the field measured tree's base location, where a lidar derived treetop of approximated tree base must fall within a specified distance of this location to be considered as matching. Our study utilizes tree base locations in lieu of the alternative, the treetop.

The value chosen for the location distance has varied within the literature (Pirotti 2010, Kaartinen et al. 2012, Vauhkonen et at al. 2012, Esyn et al. 2015). We used several different values, including Pirotti et al.'s (2010) $2.3 \mathrm{~m}$ matching distance, as well as our own $4.5 \mathrm{~m}$ matching distance, which was determined by adding Pirotti et al.’s (2010) $2.3 \mathrm{~m}$ matching distance to the rounded amount of position error in our point cloud $(2.2 \mathrm{~m})$. This $4.5 \mathrm{~m}$ matching distance is more restrictive than that of Kaartinen et al.'s (2012) 5 m matching distance. 
We refer to all these items, treetops and our approximated base locations, collectively as "matching features" since they are used to match with the field collected tree base GPS locations.

Trees that met both the height and location requirements were counted as true positives. Surveyed trees which could not be matched by a lidar tree, were counted as false negatives, type II errors, or errors of Omission. Lidar -derived trees which did not match a field tree were counted as false positives, type I errors, or errors of Commission. Other metrics calculated were the observed agreement $\left(\mathrm{Pr}_{\mathrm{a}}\right)$, commission error rate $\left(\mathrm{C}_{\text {err }}\right)$, omission error rate $\left(\mathrm{O}_{\text {err }}\right)$, chance agreement $\left(\mathrm{Pr}_{\mathrm{e}}\right)$, and the Kappa agreement statistic $(\mathrm{K})$ for the agreement of the two data sets (Pirotti et al. 2017, Esyn et al. 2015, Pirotti 2010).

$$
\begin{aligned}
& \operatorname{Pr}_{\mathrm{a}}=(\text { Number of trees matched }) /(\text { Number of trees surveyed }) \\
& \mathrm{C}_{\text {err }}=(\text { False Positives }) /(\text { True Positives }+ \text { False Positives }+ \text { False Negatives }) \\
& \mathrm{O}_{\text {err }}=(\text { False Negatives }) /(\text { True Positives }+ \text { False Positives }+ \text { False Negatives }) \\
& \operatorname{Pr}_{\mathrm{e}}=\mathrm{C}_{\text {err }}^{2}+\mathrm{O}_{\text {err }}^{2} \\
& \mathrm{~K}=\left(\operatorname{Pr}_{\mathrm{a}}-\operatorname{Pr}_{\mathrm{e}}\right) /\left(1-\mathrm{Pr}_{\mathrm{e}}\right)
\end{aligned}
$$

Once tree matching was completed for every model and the matched tree attribute tables were exported from ArcGIS Pro as .csv files, the files were combined using R statistical software, before final data analysis in JMP Pro 14.0 (R Core Team, SAS Institute).

\section{Risk Analysis}

In this section we describe our process for the identification of NERC Category 2 and 3 "fall-in" vegetation risks from a high-density UAS-lidar point cloud (NERC FAC-003). These are trees which, should they fall, possess the height to contact the electric conductor. Therefore, 
the "Threat Detection" workflow was designed to determine which trees can come into contact with electrical conductors, while considering the differences in location, height, and elevation, between the tree and electrical infrastructure. We then used our threat detection results along with other lidar -derived tree metrics to adapt the International Society of Arboriculture's tree risk assessment and utility tree risk assessment best management practices to our lidar-based measures (Smiley et al. 2017, Goodfellow 2020). The ISA's tree risk assessment BMP method's matrices are listed in Table 1 and Table 2.

Table 1: ISA tree risk assessment Likelihood Matrix

\begin{tabular}{|l|l|l|l|l|}
\hline Likelihood of & \multicolumn{4}{|c|}{ Likelihood of Impacting Target } \\
Failure & Very Low & Low & Medium & High \\
\hline Imminent & Unlikely & Somewhat likely & Likely & Very Likely \\
\hline Probable & Unlikely & Unlikely & Somewhat likely & Likely \\
\hline Possible & Unlikely & Unlikely & Unlikely & Somewhat likely \\
\hline Improbable & Unlikely & Unlikely & Unlikely & Unlikely \\
\hline
\end{tabular}

Table 2: ISA tree risk assessment Final Risk Matrix

\begin{tabular}{|l|l|l|l|l|}
\hline Likelihood of & \multicolumn{4}{|c|}{ Consequences of Failure } \\
failure and impact & Negligible & Minor & Significant & Severe \\
\hline Very Likely & Low & Moderate & High & Extreme \\
\hline Likely & Low & Moderate & High & High \\
\hline Somewhat Likely & Low & Low & Moderate & Moderate \\
\hline Unlikely & Low & Low & Low & Low \\
\hline
\end{tabular}

\section{Threat Detection}

After trees have been segmented, each tree is assessed to determine if it possesses the potential to contact the electrical conductor. We refer to this process as "Threat Detection" and each tree's height and base location, the line height and line location, and the perpendicular distance between the tree and line, as well as the elevation difference between tree and line must all be accounted for during this analysis. First, to aid in the visualization of this process, imagine a circle with a radius equivalent to the tree height circumscribed about the tree base location. If 
this circle intersects with the electric line, then the tree poses a threat of coming into contact with the electrical conductor. However, this simple visual doesn't account for the elevation difference between the two.

Our Threat Detection workflow is summarized in Figure 22. The outputs from our implementations of Li et al.'s (2012) tree detection and segmentation routine were utilized for the identification and assessment of NERC Category $2 \& 3$ vegetation risks. Once points were ascribed a "treeID" using lidR, a spatial points file for the detected treetops and a spatial polygons file for the tree crowns was imported into ArcGIS PRO for risk assessment.

Using the two spatial outputs from $\mathrm{R}$, the next step of the assessment is to determine each tree base's location. For trees with excurrent architecture, base location and top location may be in roughly the same location $(\mathrm{x}, \mathrm{y})$. In contrast, decurrent trees may experience phototropism, branch failure, and/or adaptive growth, which can cause differences in the location of the highest point and the base location of the tree. Furthermore, in the central Appalachian hardwood forest many trees tend to be decurrent when growing in the open. Thus, this study used two methods for estimating a given tree's base location. The first utilized R, where the mean X and mean Y were computed for all points with a given "treeID", before they were written out to the tree crowns spatial polygon file. This method is referred to as the " $\mathrm{XY}$ " method. The second used the ArcGIS PRO function, "Feature to Points", to identify the centroid of each tree crown polygon. This method is referred to as the "CP" method. However, both methods are conceptually flawed. For the XY method, an issue is that the distribution of lidar laser pulses within each tree is not uniform; some parts of trees may receive more lidar pulses than other parts, causing a displacement of the estimated base towards the part of the tree that received the most pulses. On the other hand, the $\mathrm{CP}$ method does not consider any information from the point cloud about the 
distribution of points inside the tree crown polygon. See Figure 23 for a visualization of the difference in approximated tree bases.

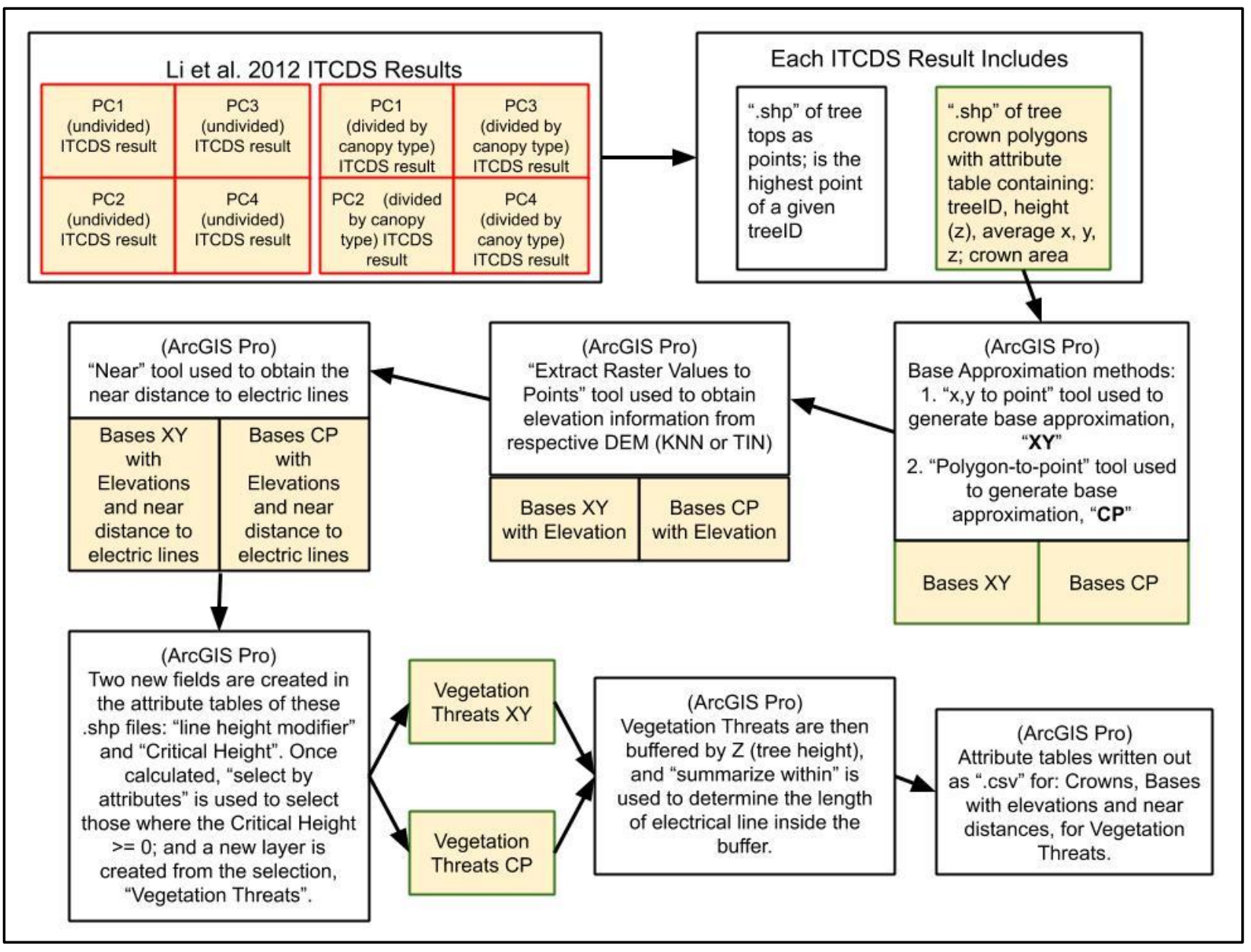

Figure 22: Threat Detection Workflow 




Figure 23: Differences in tree base estimation methods using the XY and CP methods 
The "Near" tool was used to evaluate the nearest point on the electric line to each tree base location, as well as provide the $\mathrm{x}, \mathrm{y}$ location of the nearest point on the electric line, see Figure 24. Then, a new point shapefile is generated using the function, "X,Y Table to Points", such that the nearest point on the line associated with a tree is now its own layer. Since our scan failed to detect the electric lines, a "Z" value, or height above ground of $9 \mathrm{~m}$ (30 feet), representing a typical height of the electric distribution lines, was assigned to all these nearest point locations.

Since the lines are set to one height, they lack the actual line sag, but instead simply follow the topography of the DEM, though offset by $9 \mathrm{~m}$. Had the lines been detected by the lidar sensor, then the height above ground for points classified as "electrical conductor" would be used.

To account for the elevation differences of trees and the electrical infrastructure, the "Extract Values to Points" tool is used to extract DEM elevation values for both tree base locations and their associated nearest point locations along the electrical conductor. Two new fields were computed within the tree base shapefile attributes. First, the "Line Height Modifier" is calculated and then included in the calculation of the "Critical Height". The Line Height Modifier was computed as:

$$
\text { line height }(z)+(\text { elevation at near point - elevation at tree base) }
$$

The Critical Height was computed as:

tree height $(z)$ - (distance between tree base and nearest point on the line + line height modifier)

Lastly, the "Select by Attributes" function was used to return all tree bases where the Critical Height was greater than or equal to zero. This selection represents all NERC category 2 and 3 vegetation threats, and accounts for the elevation differences between the tree and the 
electrical infrastructure. See Figure 25 for a visualization of the matched NERC Category $2 \& 3$ trees. 


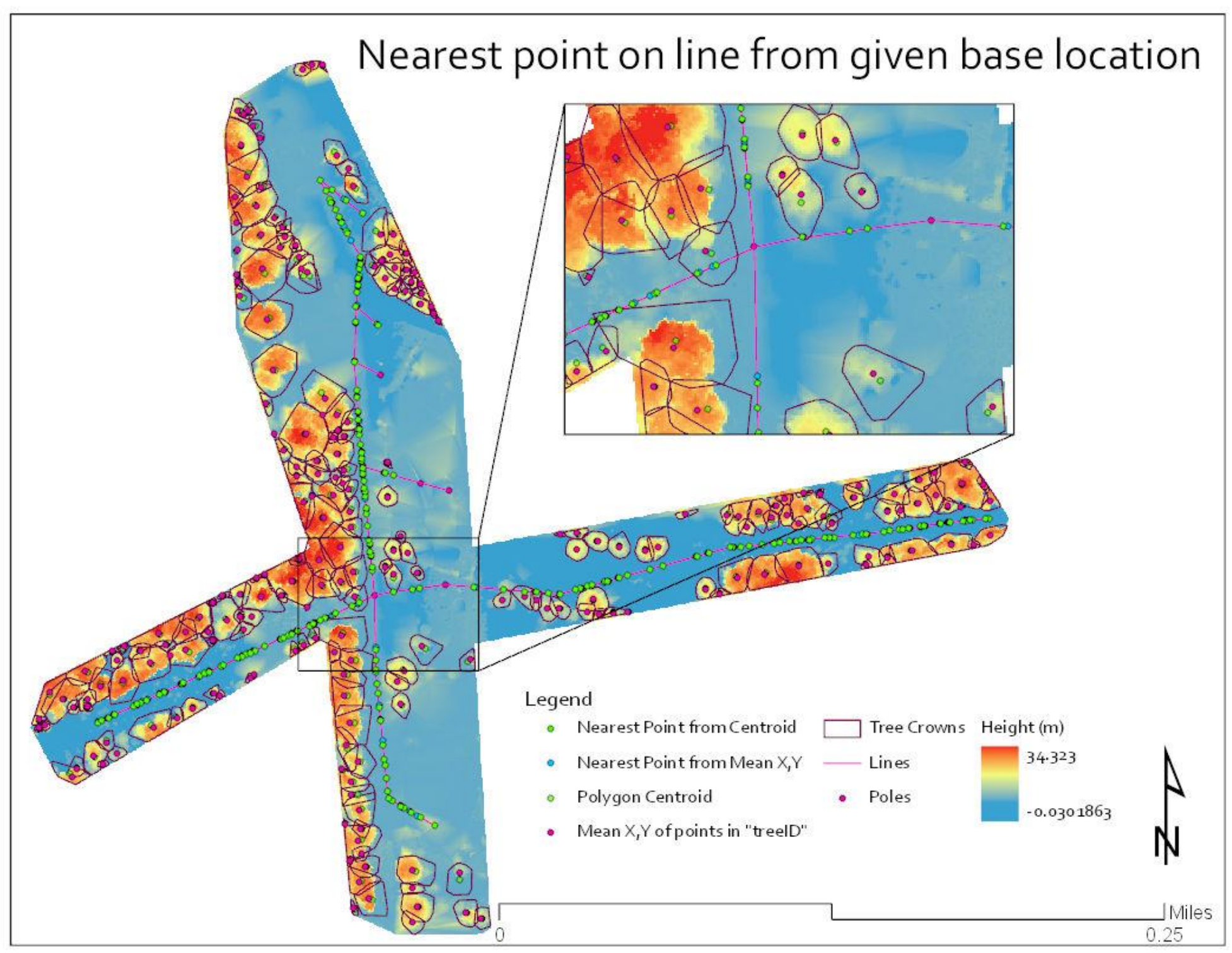

Figure 24: Nearest point on the electric line from tree base locations 


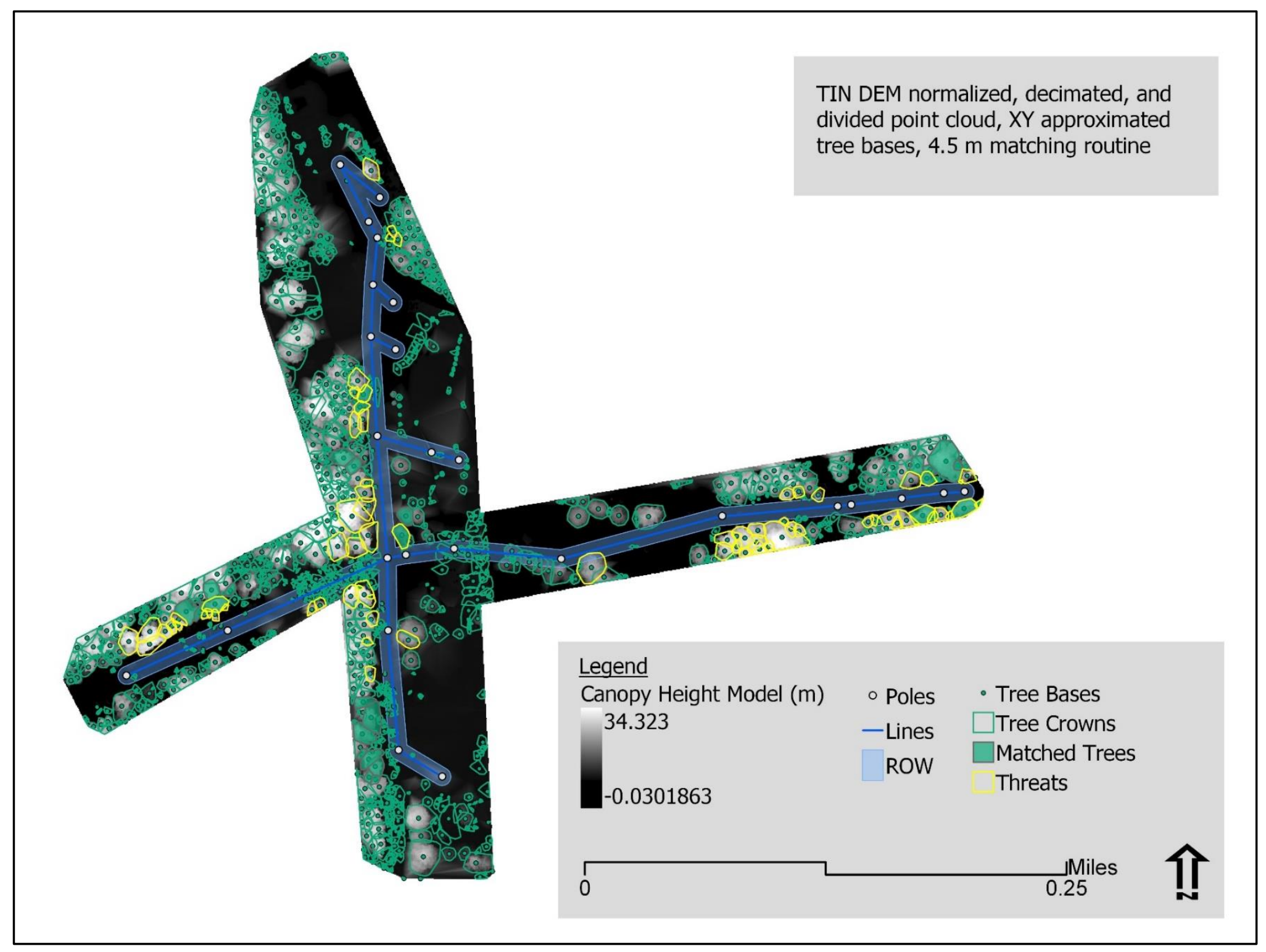

Figure 25: Matched Trees and NERC FAC-003 Category 2 \& 3 Vegetation Threats 
ISA Tree Risk Assessment Classification

After each individual tree detection and segmentation routine and the Threat Detection workflow, we used our lidar derived information for tree risk assessment of the trees determined to be threats. We adapted the ISA's Tree Risk Assessment BMP method's double matrix approach to our lidar derived information and the available arboricultural knowledge of physical properties of trees and their relationships with tree failure. The ISA's tree risk assessment matrices are available in Table 1 and Table 2. The ISA's tree risk assessment BMP method was designed to be utilized by ISA Tree Risk Assessment Qualified (TRAQ) arborist for a qualitative assessment of tree risk (Smiley et al. 2017). Given the quantitative nature of our lidar -derived tree properties, this required the classification of our quantitative tree risk assessment measures into the categories presented in the ISA's tree risk assessment BMPs. The following sections will detail each of the classification processes.

\section{Likelihood of Failure}

For the calculation of each lidar -derived tree's Likelihood of Failure measure, we used the interaction of tree height $(\mathrm{Z})$ and $\mathrm{DBH}$ because diameter and height have been two of the more consistent variables for predicting tree failure in the arboricultural literature (Kane 2008, Peterson 2007, Gardiner et al. 2008, Dahle et al. 2017). Yet, lidar does not directly provide DBH. This study had originally intended to use an adaptation of Hagan and Smith's (1986) method for tree DBH prediction from crown area. Yet, when utilized this method with our lidar -derived data, the coefficients provided in their study produced negative values for some of the predicted DBHs. This in turn resulted in negative values for our Likelihood of Failure measure. So, a new set of models were developed for predicting DBH from UAS-based lidar derived tree metrics. 
The subset of data that was used for the generation of new DBH prediction models was the point cloud where: the AOI was divided based upon vegetation canopy type, the TIN DEM was used for point cloud normalization, the point density was decimated to $50 \mathrm{ppm}^{2}$, the matching feature were the treetops and the matching method used Esyn et al.'s (2015) height adaptive matching distance. This model matched 28 of our trees field trees and was used to develop the DBH prediction formula. We utilized JMP Pro 14.0 to fit models and thus to obtain fit statistics and the expression formula for each model. In all, we fit three models in addition to the adaptation of Hagan \& Smith's (1986) method. The $\mathrm{R}^{2}$, Adjusted R ${ }^{2}$, and RMSE are provided for the fit of each of the models to field measured DBH in Table 3.

Using JMP's “Fit Model” and "Show prediction expression”, we obtained the prediction formula for "New Predicted DBH \#1" where the effects were tree height (Z), Crown area, and the interaction of tree height and crown area.

$$
\begin{aligned}
Y= & (-9.733393443)+(1.0972323732 * Z)+(-0.023312172 * \text { Crown Area })+ \\
& (Z-24.278464286) *((\text { Crown Area }-94.164704929) * 0.0057751524)
\end{aligned}
$$

Our "New Predicted DBH \#1" model provided the best fit against the 28 matched trees in our subset. next, we considered how well each model fit against the remaining matched trees from the other point clouds, see Table 4.

Once each tree's diameter has been predicted from crown area and tree height, the predicted diameter value is multiplied by tree height to obtain a surrogate measure to stand in for the actual Likelihood of Failure. Figure 26 shows the resulting distribution of our likelihood of failure measure as calculated by height multiplied by $\mathrm{DBH}$, for matched trees across all methods. We recoded the values into categories from the ISA's tree risk assessment BMPs (Smiley et al. 2017). Figure 27 displays the if/then conditional statements used classify the likelihood of failure 
measure into categories. We followed an exponential scale for the categories, using the quantiles as a guide, because it seemed appropriate given the distribution of the data, and aligned well with the author's observational experience of trees and tree failure.

Table 3: Fit Statistics for "New Predicted DBH \#1"

\begin{tabular}{|l|l|l|l|}
\hline Model & $\mathrm{R}^{2}$ & Adjusted $\mathrm{R}^{2}$ & $\mathrm{RMSE}$ \\
\hline Hagan \& Smith (1986) & 0.1543 & 0.1218 & 5.7801 \\
Where, & 0.6759 & 0.6354 & 3.7243 \\
Field Measured DBH = tree height (lidar derived: Z), & & & \\
crown area (lidar derived), and the interaction of tree & & & \\
height and crown area. & & & \\
\hline $\begin{array}{l}\text { New Predicted DBH \#2 | Height and Crown Area Model } \\
\text { Where, }\end{array}$ & 0.5152 & 0.4764 & \\
Field Measured DBH = tree height (lidar derived: Z) and & & & \\
crown area (lidar derived) & & & \\
\hline $\begin{array}{l}\text { Wield Measured DBH = tree height (lidar derived: Z) } \\
\text { Where, }\end{array}$ & & & \\
\hline
\end{tabular}


Table 4: Prediction Statistics for "New Predicted DBH \#1"

\begin{tabular}{|c|c|c|c|}
\hline Model & $\mathrm{R}^{2}$ & Adjusted $\mathrm{R}^{2}$ & RMSE \\
\hline Hagan \& Smith (1986) & 0.2222 & 0.2217 & 5.5282 \\
\hline $\begin{array}{l}\text { New Predicted DBH \#1 | Full Model (with } \\
\text { interaction) } \\
\text { Where, } \\
\text { Field Measured DBH = tree height (lidar } \\
\text { derived: Z), crown area (lidar derived), and } \\
\text { the interaction of tree height and crown area. }\end{array}$ & 0.54488 & 0.5446 & 4.2289 \\
\hline $\begin{array}{l}\text { New Predicted DBH \#2 | Height and Crown } \\
\text { Area Model } \\
\text { Where, } \\
\text { Field Measured DBH = tree height (lidar } \\
\text { derived: Z) and crown area (lidar derived) }\end{array}$ & 0.4967 & 0.4963 & 4.4471 \\
\hline $\begin{array}{l}\text { New Predicted DBH \#3 | Height Model } \\
\text { Where, } \\
\text { Field Measured DBH = tree height (lidar } \\
\text { derived: Z) }\end{array}$ & 0.4542 & 0.4538 & 4.6310 \\
\hline
\end{tabular}




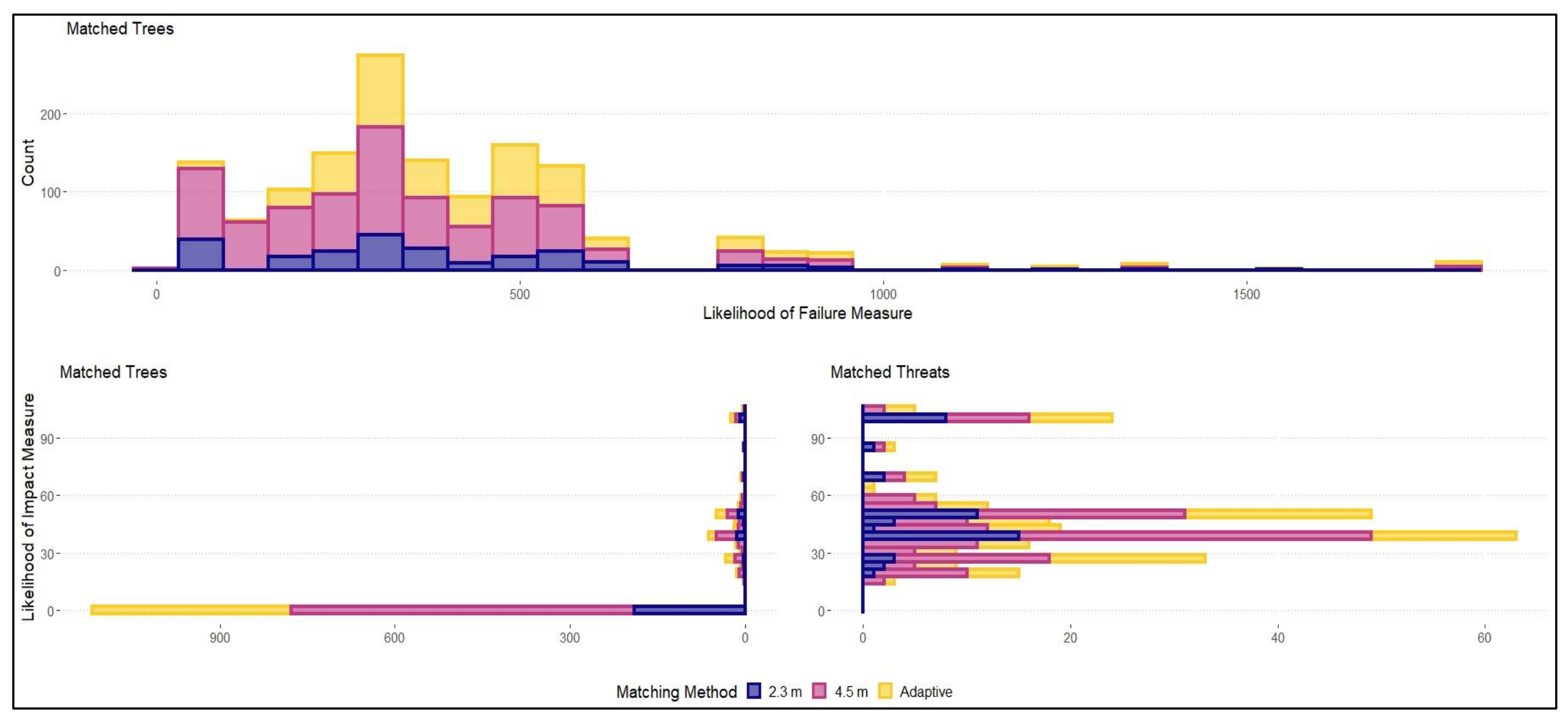

Figure 26: The distributions of the Likelihood of and Likelihood of Impact measures for all matched trees and the Likelihood of Impact measure for matched trees determined to be vegetation threats 


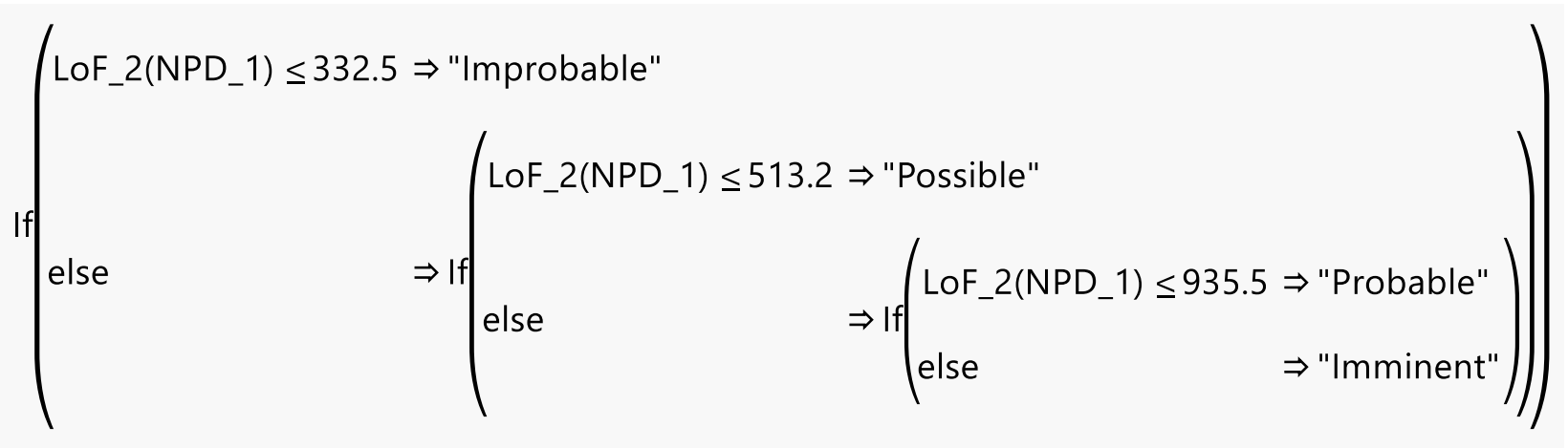

Figure 27: Recode formula for Likelihood of Failure Measure to ISA's Likelihood of Failure Categories

this model of Likelihood of Failure measure provides an approximation of the true value. Until a more formalized method of predicting the likelihood of individual tree failure is possible from remotely sensed data, models such as GALES, HWIND, or FOREOLE could also be incorporated in concert with the ISA's tree risk assessment BMPs. See Figure 28 for choropleth map of tree crowns by their Likelihood of Failure classification and Figure 29 to see matched trees by their Likelihood of Failure Classification. 


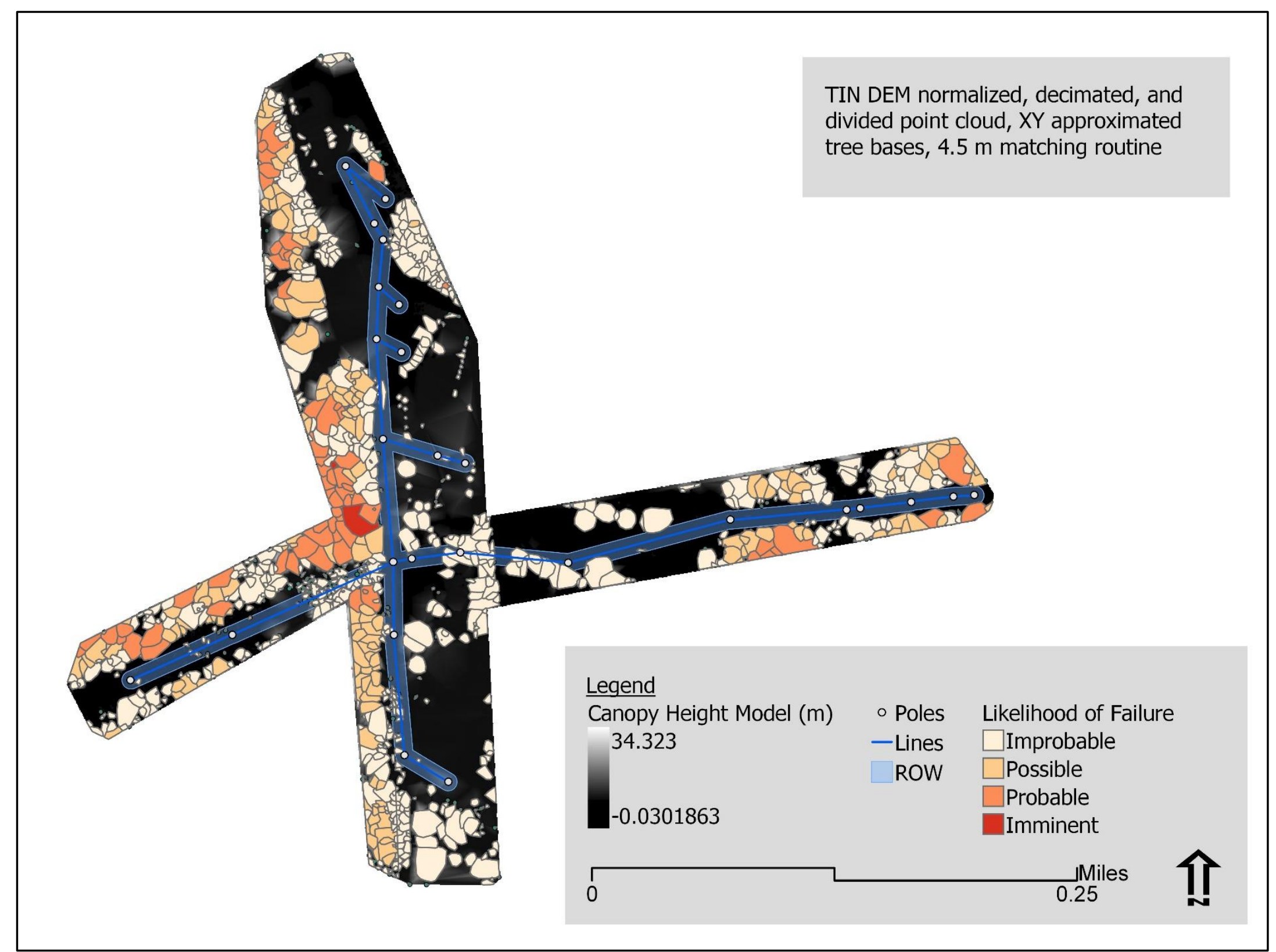

Figure 28: Likelihood of Failure Classification 


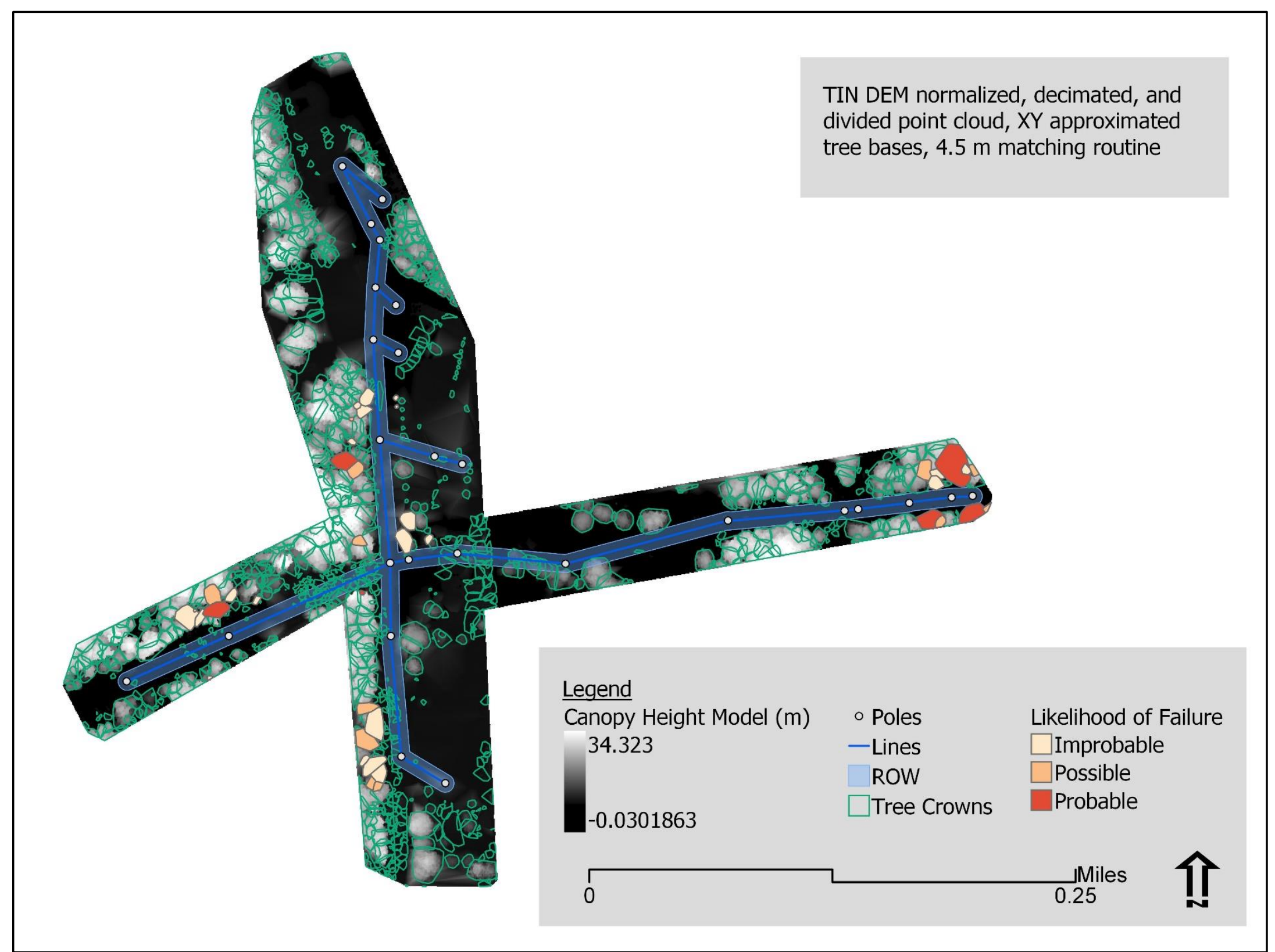

Figure 29: Likelihood of Failure classification for Matched Trees 


\section{Likelihood of Impact}

For the calculation of the Likelihood of Impact, we utilized a buffer around each lidar derived tree's approximated base location that was equivalent to its height. Then, we calculated the length of electric line that fell inside that buffer to provide scale for the Likelihood of Impact measure, see Figure 31. For trees of equal height, those with a longer length of line inside their buffer region have a larger proportion of the potential direction of fall that would result in contact with the line. thus, these trees are more likely to impact the line.

The distribution of the Likelihood of Impact variable for the study area is provided in Figure 26. The Likelihood of Impact measures were recoded into the ISA tree risk assessment BMP's four categories, see Figure 30 for the if/then conditional statements used to classify the Likelihood of Impact values into categories. The categories mimic the quartiles for the distribution of matched trees which were deemed vegetation threats, such that 34.5 is the upper limit of the lowest quartile, 41.06 is the upper limit of the second quartile, and 51.27 is the upper limit of the third quartile.

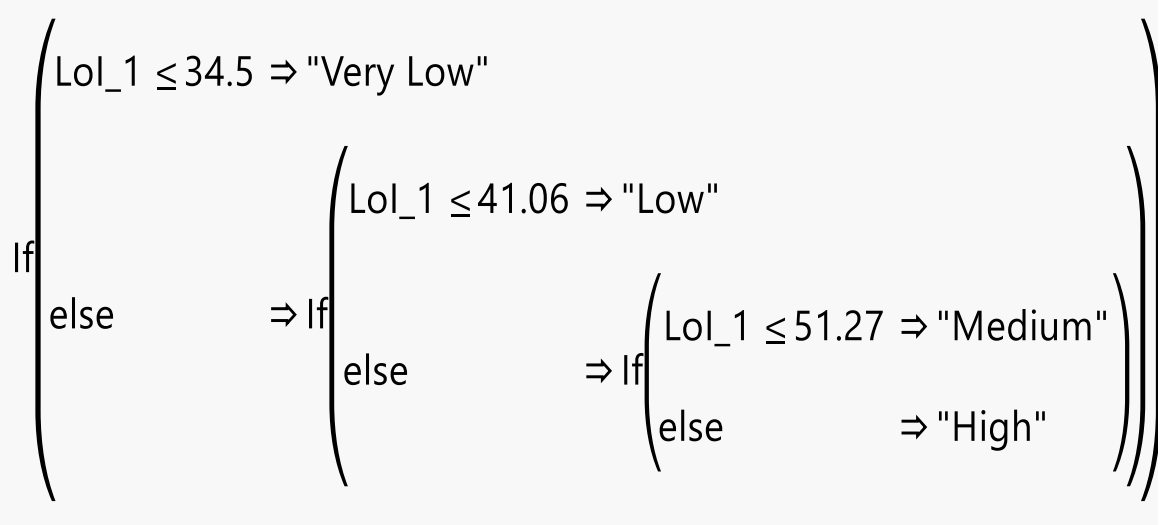

\section{Figure 30: Likelihood of Impact Classification formula}

In the future, this method for approximating the likelihood of impact should be refined, for example by calculating the range of angles which could result in impact, because length of 
line alone will not always accurately approximate the potential range of angles, see upper left inset image in Figure 31. The tree in this inset image will have its Likelihood of Impact overestimated based on the current methodology. The tree crown polygons representing NERC category 2 and 3 vegetation risks were then displayed in a graduated choropleth of the Likelihood of Impact classification, see Figure 32. For the Likelihood of Impact classification of matched trees see Figure 33. 




Figure 31: Likelihood of Impact Measure Calculation 




Figure 32: Likelihood of Impact classification for trees identified as within the striking distance of the distribution powerline. 


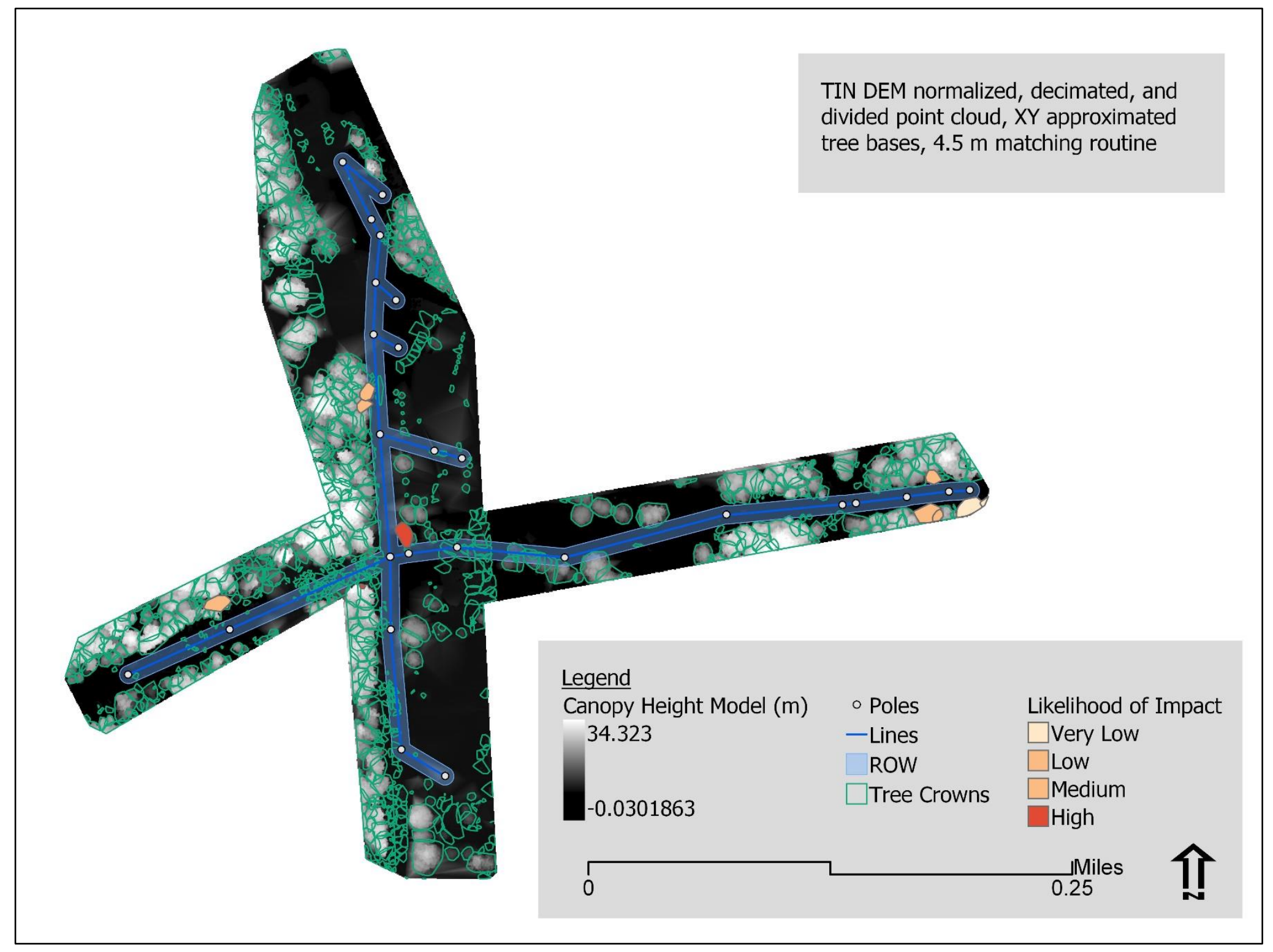

Figure 33: Likelihood of Impact classification for matched trees 


\section{Final Risk Rating}

The International Society of Arboriculture's Tree Risk Assessment method utilizes a pair of matrices to calculate a qualitative Final Risk Rating (Smiley et al. 2017, Goodfellow 2020). These matrices are reproduced in Table 1 and Table 2. The first matrix, the "Likelihood Matrix", combines the likelihood of failure and the likelihood of impact into a singular likelihood measure. This measure is then combined, in the second matrix, with a measure for the consequences of failure, to calculate the final risk rating (Smiley et al. 2017, Goodfellow 2020).

Our study used the interaction of tree height and DBH as a surrogate measure for Likelihood of Failure because the combination of the two has been found to correlate well with tree failures in arboricultural post-storm literature (Kane 2008, Gardiner et al. 2008, Peterson 2007, Francis \& Gillespie 1993, Duryea et al. 2007b). Furthermore, we calculated the Likelihood of Impact as the length of electric line that fell within a buffer around a tree's base equivalent to the height of the tree. These two values are then used to generate a "Likelihood Measure" for each tree, which is used as an input for the second matrix.

Likelihood of Failure and Impact measures were recoded into the ISA tree risk assessment BMP's four categories for the Likelihood Measure provided by the Likelihood Matrix, using if/then conditional statements displayed in Figure 34.

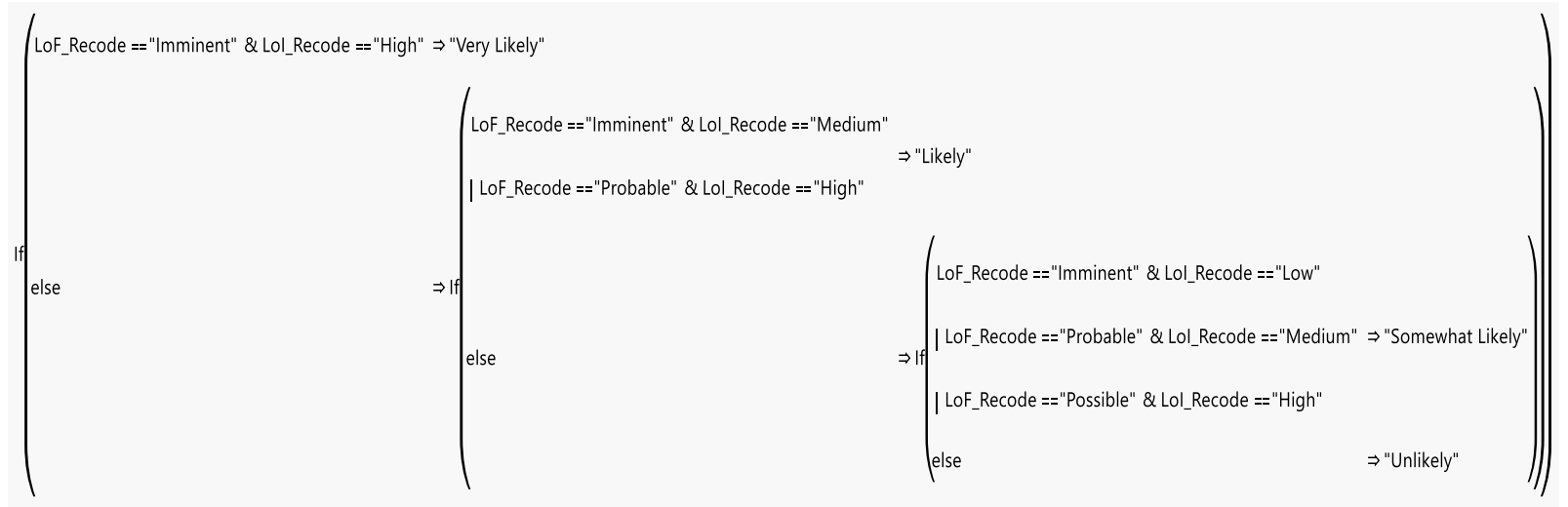

Figure 34: Likelihood Matrix classification formula 
To allow for varying levels of consequences of failure for each tree, we deployed simulated values of customer counts to different segments of our modeled electrical lines (Figure 35). This grid model was held constant, and thus, used with the results from each individual tree detection and segmentation.



Figure 35: Simulated Electrical grid customer count model

Consequences of Impact were recoded from the "Customer Number" field within the attribute table of the "Lines" shapefile, see Figure 36. Final risk ratings for each tree could then be calculated as the Likelihood Measure combined with the Consequences of Impact, using the if/then conditional statements available in Figure 37. A choropleth representing the Final Risk 
Rating classification of trees deemed to be vegetation threats is shown in Figure 38, Along with a choropleth of the Final Risk Rating clasification of matched trees in Figure 39.



Figure 36: Consequences of Impact classification formula

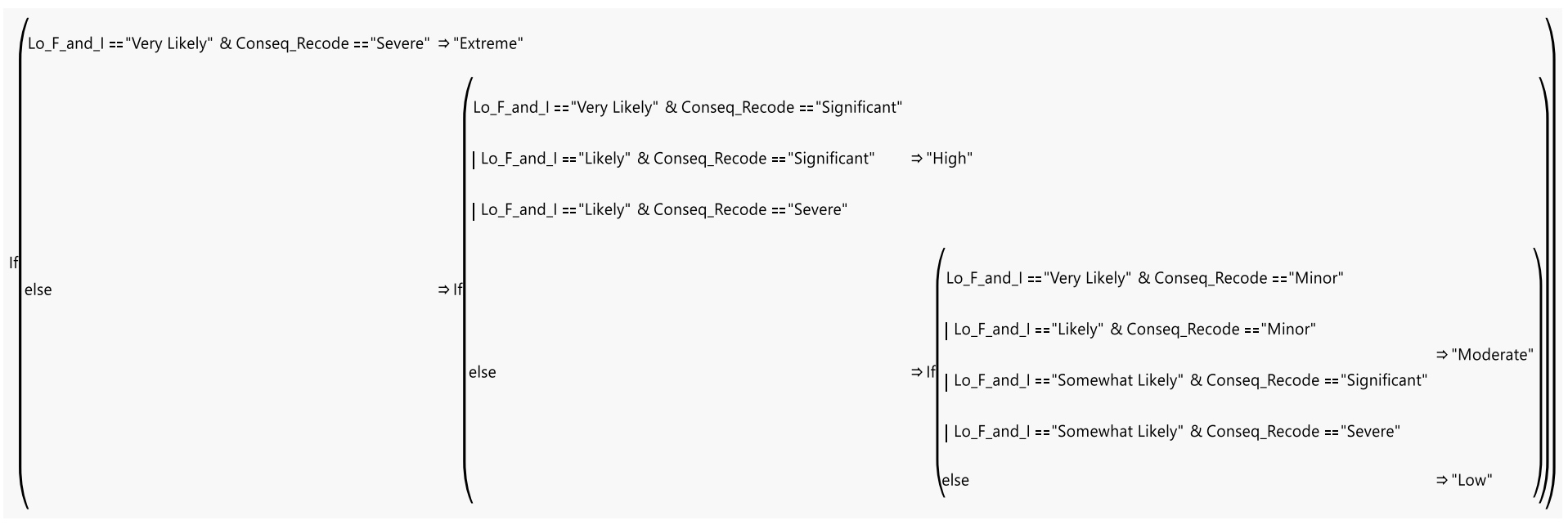

Figure 37: Final Risk Rating classification formula 


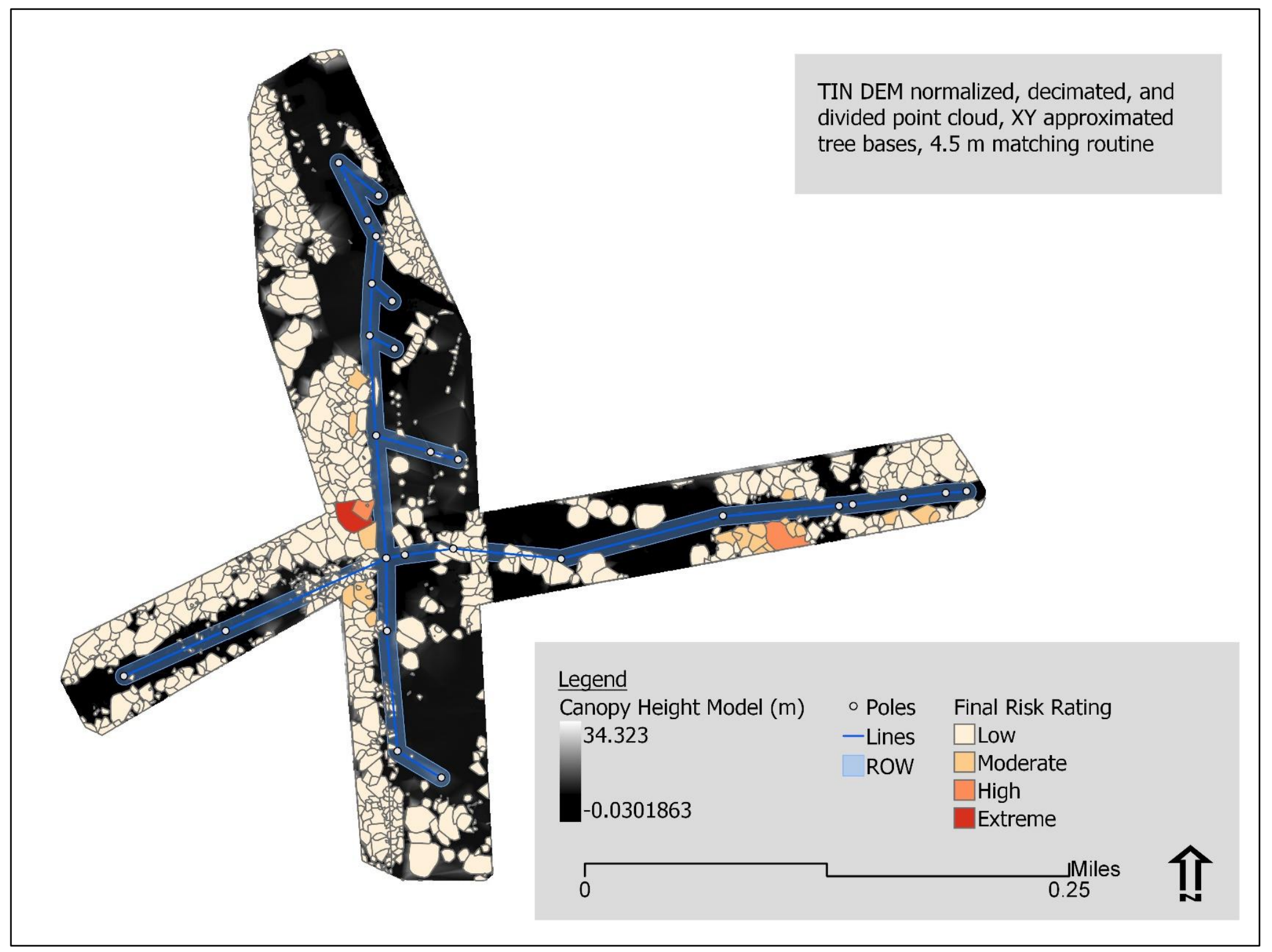

Figure 38: Final Risk Rating classification 


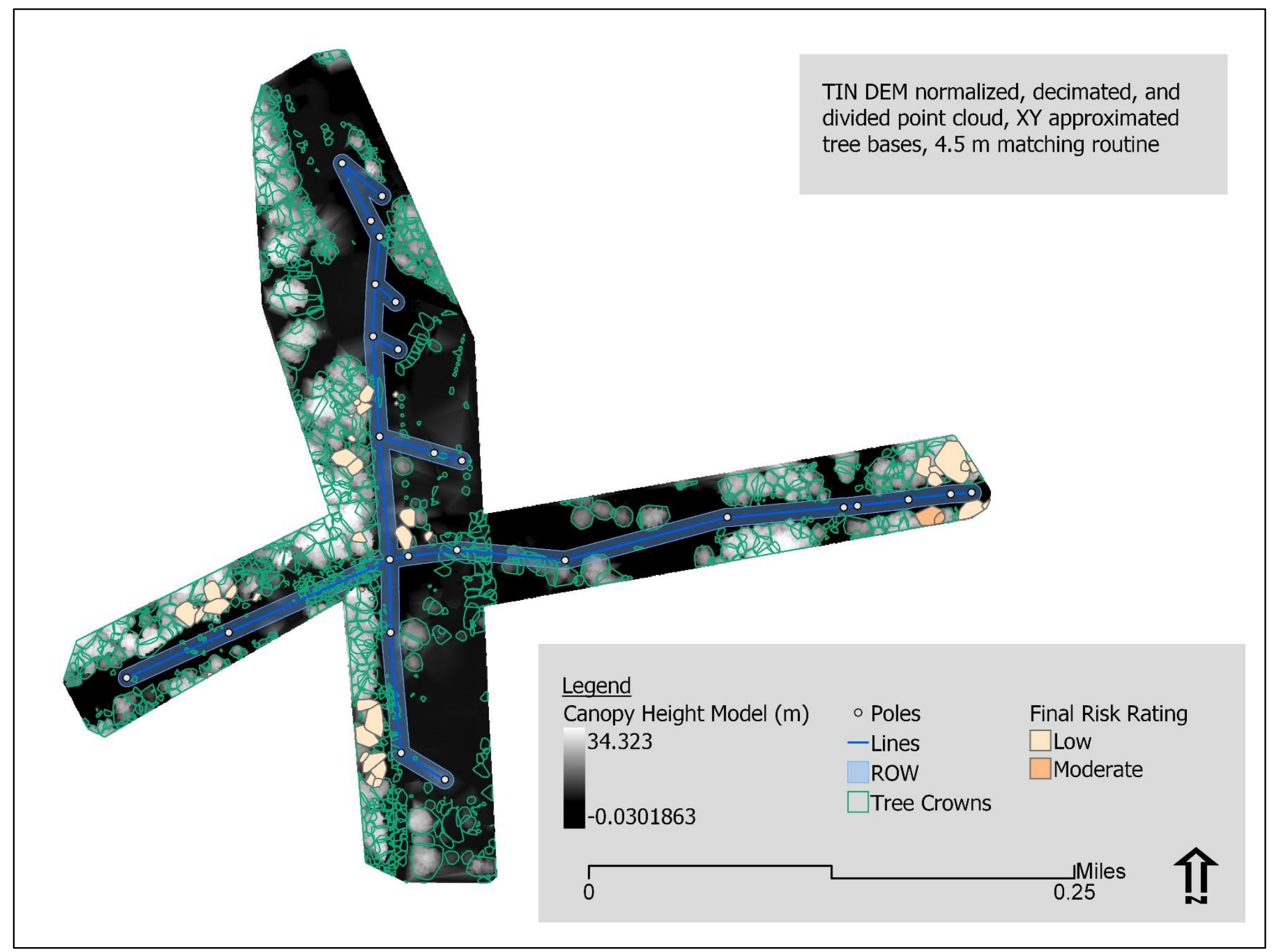

Figure 39: Final Risk Rating classification of matched trees 


\section{Field Data}

two attempts were made for field data collection for model validation. The first was

abandoned due to gross error in early individual tree detection and segmentation models. a

subsequent campaign completed the field data collection. A visualization of our field data and

validation workflow is displayed in Figure 40.

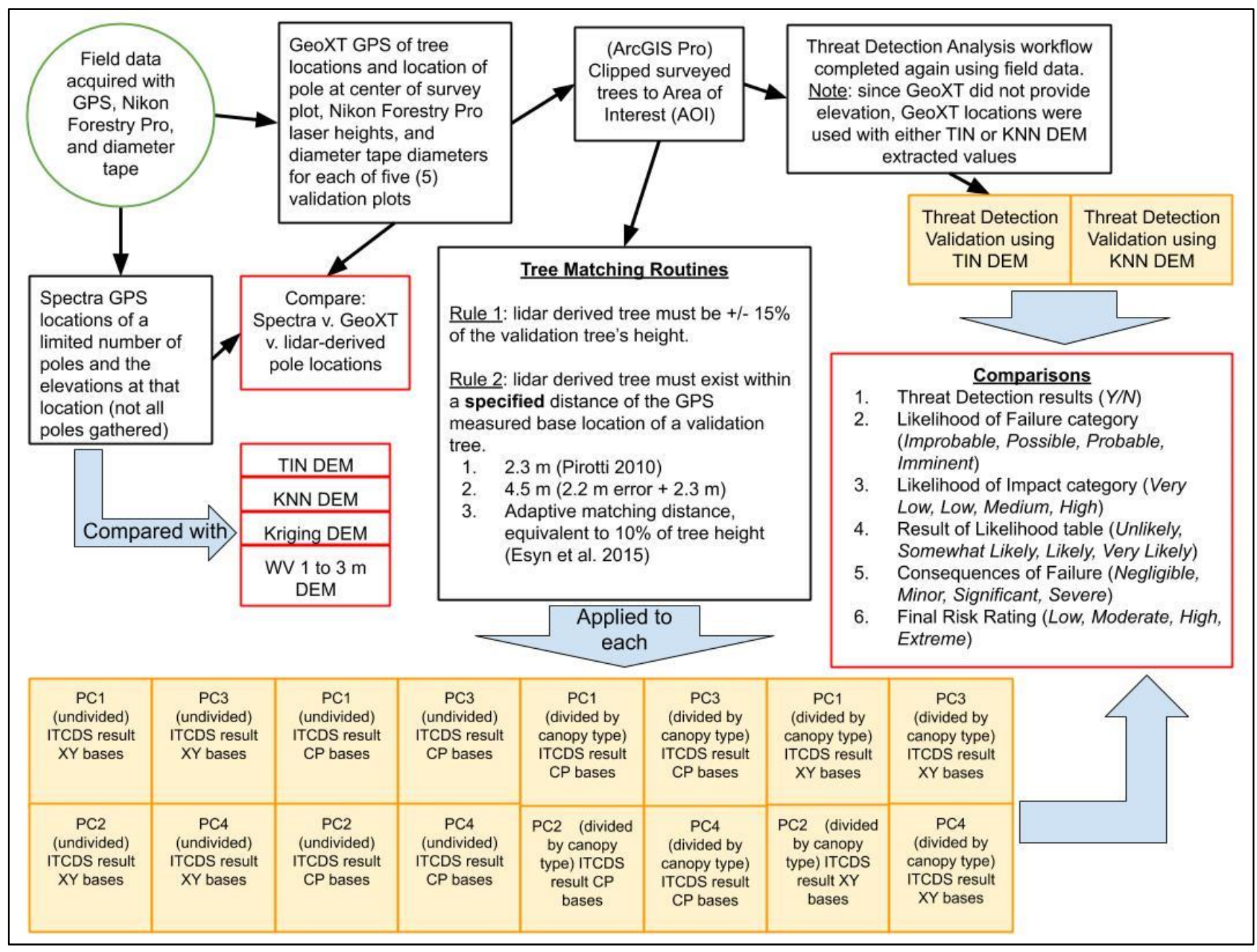

Figure 40: Field data and Validation Workflow

The first field data campaign attempted to utilize random, circular plot sampling, where

random points were generated in ArcGIS PRO. Each plot was to contain a minimum of 3

segmented trees bases, where we would assess location, elevation, diameter at breast height,

diameter at base, tree height, and tree lean, for all stems of at least 1-inch in DBH. Additionally, 
to validate elevation accuracy and location accuracy in the point cloud, location and elevation were assessed at several points along a road within the scan area.

We intended to complete a minimum of 15 plots, but after data collection for the first three plots, it became obvious that adjustments to the tree detection and segmentation algorithm were required. Each of the plots were predicted by our models to contain 3 trees. Yet, our three completed field plots contained 9, 18, and 50 trees. This large degree of error indicated gross under-segmentation and some error of omission. Minor omission errors of understory trees were expected, but errors of this magnitude were not. Thus, the first field data collection was aborted, and more time and research were devoted to exploring the tree segmentation process and results.

The second field data collection used a different methodology than the first collection, namely a systematic sampling of trees in five circular plots. Each plot was centered on a utility pole, and all vegetation greater than 1-inch DBH within $30 \mathrm{~m}$ of the pole was assessed for GPS location, height, and diameter. The plots were placed at either end of each of the flight lines within the AOI, and one plot was placed at the intersection of the two lines. See Figure 6 for the location of the five plots.

We dropped the measurement of diameter at tree base because diameter at breast height was already being assessed and additional diameter data was deemed redundant. Additionally, the tree lean measurement was dropped due to lack of interpretability, as lean angle became difficult to assess both in the field and within the point cloud. Future research should be devoted to tree lean measurement from lidar to determine if trees are leaning towards or away from electrical infrastructure, as this would play a pivotal role in the risk presented to electrical infrastructure by the vegetation. 
A Nikon Forestry Pro handheld laser was used for height measurements in meters (m) and a handheld diameter tape used for the diameter measurement in inches (in). Additionally, the two separate attempts at gathering field data utilized two separate GPS receivers. The first used a survey grade Spectra SP80 unit, which could provide elevation data for the gathered points. The latter attempt used a Trimble GeoXT handheld which could not provide elevation data. Both attempts used point averaging of at least 10 occupations to improve GPS accuracy.

We generated a points shapefile from the GPS locations for tree bases acquired with the Trimble GeoXT handheld GPS. Then, we used ArcGIS Pro to populate the attribute table of the point shapefile with the remaining field acquired tree data. Next, a copy of the points shapefile was made and one copy was used to "extract raster values to points" with the TIN DEM, and the other copy with the KNN DEM. Since the GeoXT could not acquire elevation data, these elevation values were used as validation values for our lidar derived tree base elevations. Additionally, these elevations were used along with the field acquired tree heights and locations to conduct the same Threat Detection and tree risk assessment classification processes as were conducted with the lidar derived trees. The heights and diameters of field trees and the results of the Threat Detection and tree risk assessment classifications from field trees are displayed in Figures 41, 42, and 43.

Next, we utilized JMP Pro 14.0 (SAS Institute) to conduct a series of contingency analyses on the classification results of Threat Detection, Likelihood of Failure, Likelihood of Impact, Likelihood Matrix, Consequences of Impact, and Final Risk Rating classifications for each model lidar derived model compared to the field dataset using the same DEM. We then estimated the Kappa agreement statistic and the associated probability $>\mathrm{z}$ for each model. Consequences of Impact were held constant across models as was necessary for examination of 
the of the Likelihood of Failure, Likelihood of Impact, Likelihood Matrix, and Final Risk Rating classifications. Thus, the Kappa agreement statistic for the Consequences of Impact classification was 1.0, or perfect agreement, regardless of model. Due to the number of models in the study, the Kappa values for each classification are displayed as distributions by DEM for normalization. 


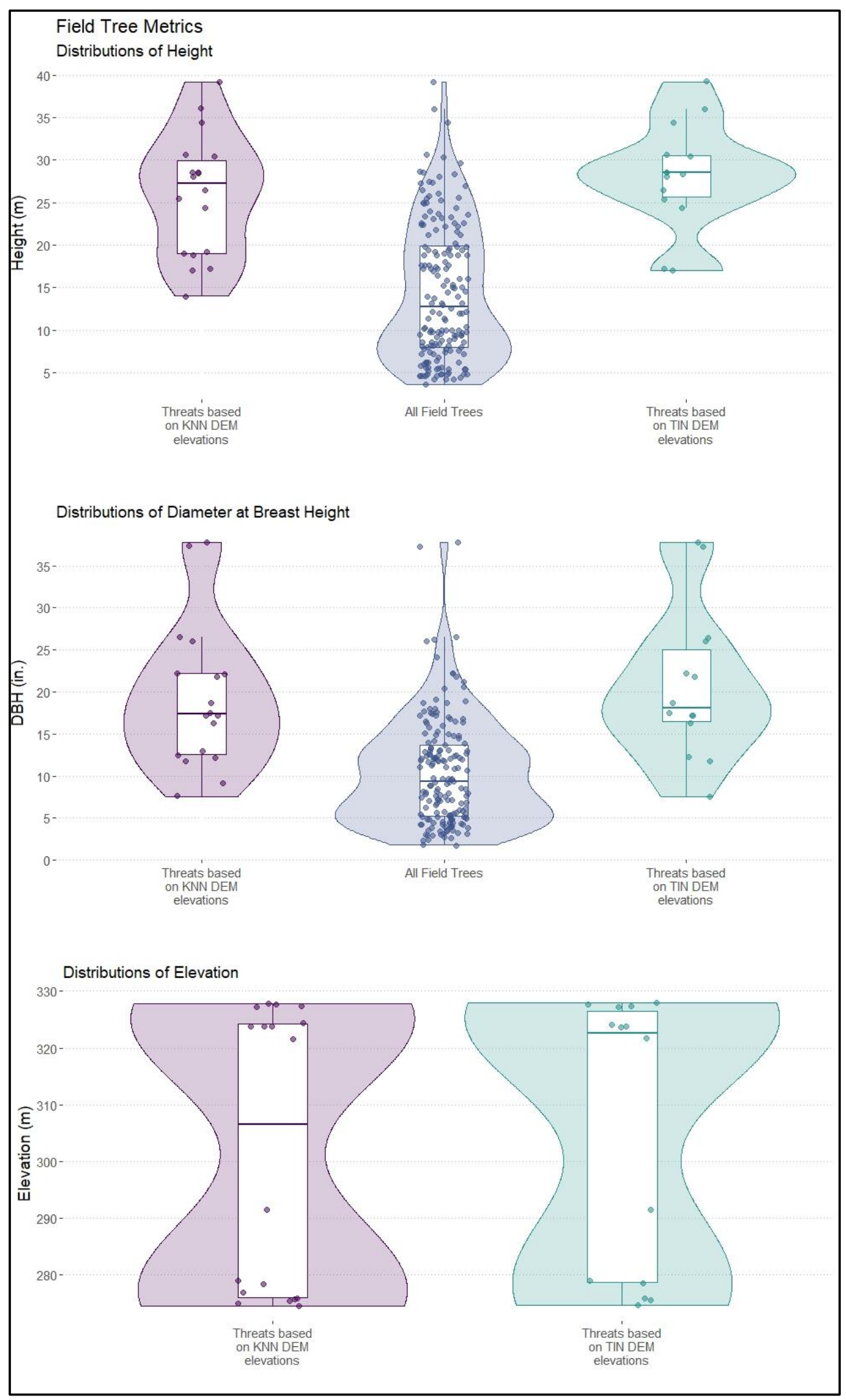

Figure 41: Field Tree Metrics: Distributions of Height, DBH, and Elevation 


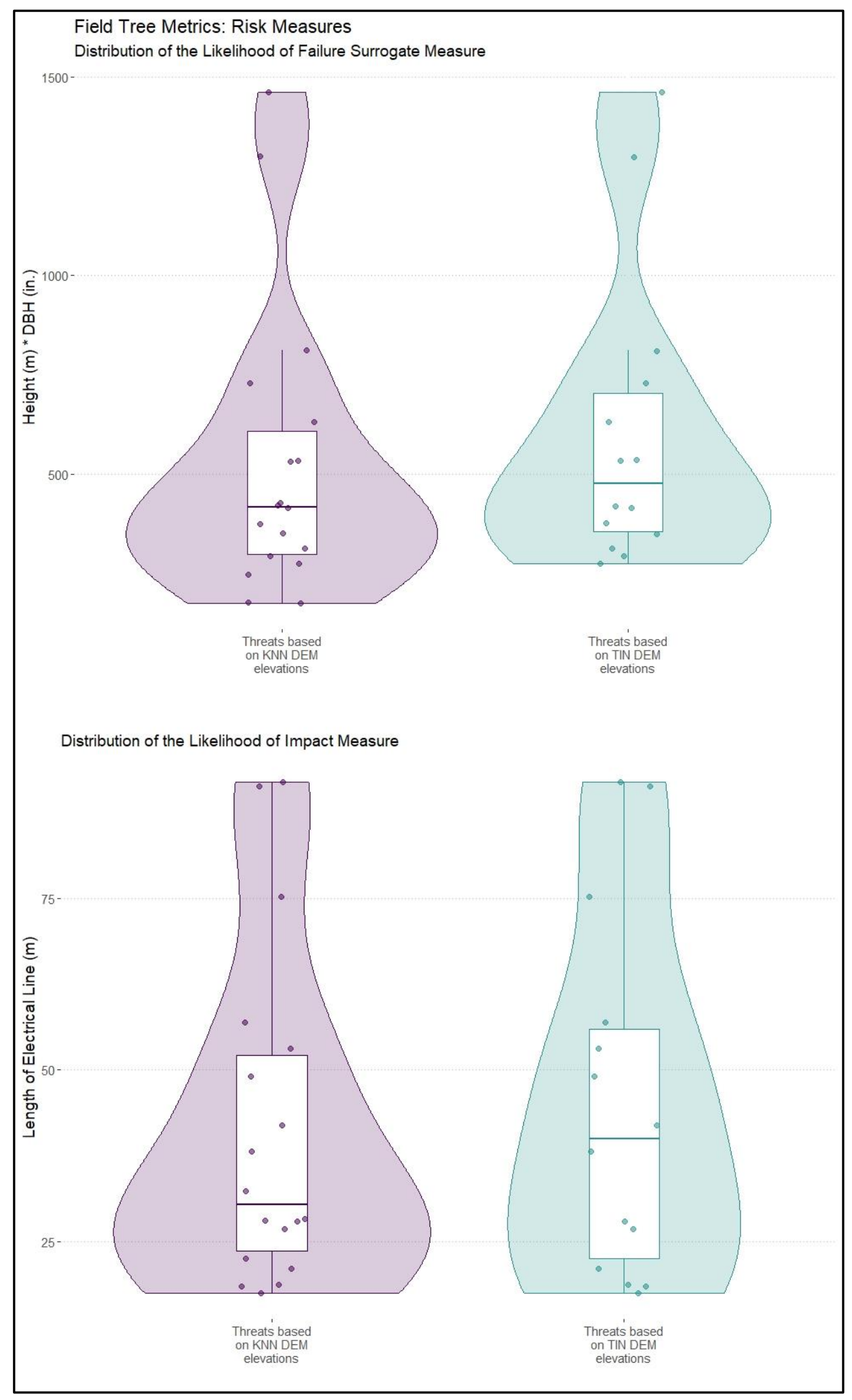

Figure 42: Distributions of Field Tree Likelihood of Failure and Likelihood of Impact Measures 


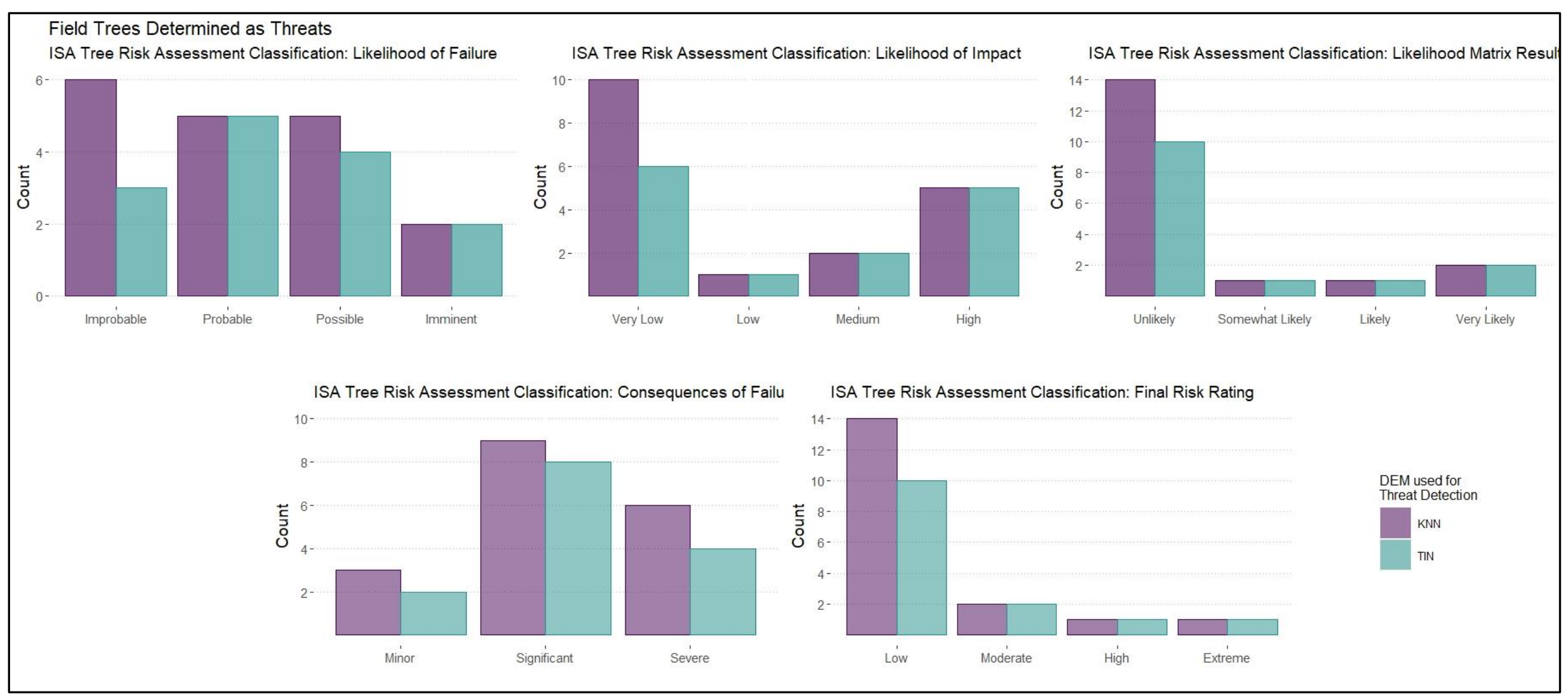

Figure 43: Field Tree Metrics: ISA Tree Risk Assessment Classification of Field Trees determined to be Threats 


\subsection{Results}

Given the complex nature of individual tree crown detection and segmentation from lidar and the relationships among tree height, location and elevation, and power line height, location and elevation; we will examine the differences in each step of the process and attempt to understand their effects later in the stages of risk analysis. We used a probability value of 0.05 to determine statistical significance throughout our analyses. This section is structured such that we will begin by looking into differences in digital elevation models (DEMs), before moving to power line pole locations. After which, we will begin our analysis of the individual tree detection and segmentation routines, with an in depth look at the performance within each tree matching routine. Lastly, we will examine our risk assessment analyses and our attempt to best adapt the ISA's tree risk assessment BMP method to our lidar based tree metrics.

\section{DEM \& Pole Locations}

As discussed earlier, this project included two separate collections of field GPS data. The first, utilized a Spectra SP80 and the second, a Trimble GeoXT. We were concerned that the lidar would be both more accurate and more precise than the GeoXT. Additionally, since the GeoXT wasn't capable of elevation measurement the validation of DEM elevations and tree base elevations became more complicated. Yet, in both cases, we developed workarounds. For the first, we concluded that to best validate DEM elevations, we would utilize the elevation data for electric poles and road points gathered during the first abandoned field data collection. For the second workaround, we decided to use the lidar derived DEM elevation values at the locations of tree bases collected during the second field data collection to compare against the lidar derived tree base elevations. 
We processed three DEMs of our study area from the lidar, each using a unique method for DEM interpolation. These methods included, a triangulated irregular network (TIN) model, an inverse distance weighted K-nearest neighbor model (KNN), and a kriging model; and all were processed to be $0.5 \mathrm{~m}$ resolution. In addition to these models, we also used the highest resolution DEM available from the state of West Virginia (WV1to3m). This DEM is a state-wide DEM with a variable resolution of between $1-3 \mathrm{~m}$, and it was clipped to the same area of interest as the other DEMs.

We used a Spectra SP80 survey grade GPS, to collect elevation data at 15 points, nine at power line poles in the study area and, six along the road. These locations were chosen because the poles were easily identifiable locations in the point cloud. Additionally, the road locations provided stable flat areas, with no overhead tree canopy which allowed for the acquisition of high accuracy elevation data.

We utilized JMP Pro 14.0 to conduct repeated measures ANOVA on the five sources of elevation data. The ANOVA revealed that there was a significantly different model in the group (p-value $=<0.0001)$. Therefore, Tukey's HSD post-hoc test for all pairwise differences was used to determine that the WV1to3m DEM was different from all other models ( $\mathrm{p}$-value $=<0.0001$ ), and that the Spectra GPS measurements were different from both the state's model (WV1to3m) 
and all the lidar derived models $(\mathrm{p}$-value $=0.0017$ ). There were no statistically significant differences among the lidar derived models, see Figure 44.

\begin{tabular}{|lrrrr|}
\hline Method & & & $\begin{array}{r}\text { Least Squares } \\
\text { Mean }\end{array}$ \\
WV1to3m & A & & & 313.77400 \\
Spectra & & B & & 281.45153 \\
KNN & & C & 279.96982 \\
Krige & & C & 279.96682 \\
TIN & & C & 279.96310 \\
\hline
\end{tabular}

Figure 44: DEM comparison: Connecting Letters Report

The "WV1to3m" DEM was dropped from further analysis as it was significantly higher in elevation than the lidar derived models and the Spectra measurements. The kriging DEM was dropped from further analysis due to visual artifacts in the DEM, as noted earlier. The TIN and KNN DEMs were retained and used to normalize the point cloud, each normalized point cloud was saved separately.

\section{Pole Locations}

Pole locations were obtained via three separate methods: Spectra SP80, Trimble GeoXT handheld GPS, and the lidar derived pole locations. The Spectra and Trimble GPS datasets included different subsets of the total poles in the area of interest, while the lidar dataset included all the poles in the dataset. Thus, comparisons between methods utilized a different number of poles, where the Spectra and Trimble shared four poles, the Spectra and the lidar shared eight poles, and the Trimble and the lidar shared five poles. The distance between two methods of pole location for a given pole ID were used as differences for comparison.

One-way ANOVA showed no statistically significant differences among the pole location differences $(p$-value $=0.4021)$. Yet, both Levene's and Bartlett's tests for equal variances were 
significant (p-value: 0.0213 and 0.0183 , respectively) suggesting that the datasets have different variances. Thus, Welch's ANOVA was used to control for different variances, while comparing means of the differences of pole locations. Welch's ANOVA, again, found no statistically significant differences in the differences of pole locations among pole locations ( $\mathrm{p}$-value $=$ 0.4104), nor did a Steel-Dwass nonparametric all pairwise comparison post-hoc (p-values: $0.8231,0.9283,0.9288)$. A visualization of the pole location differences is available in Figure 45.

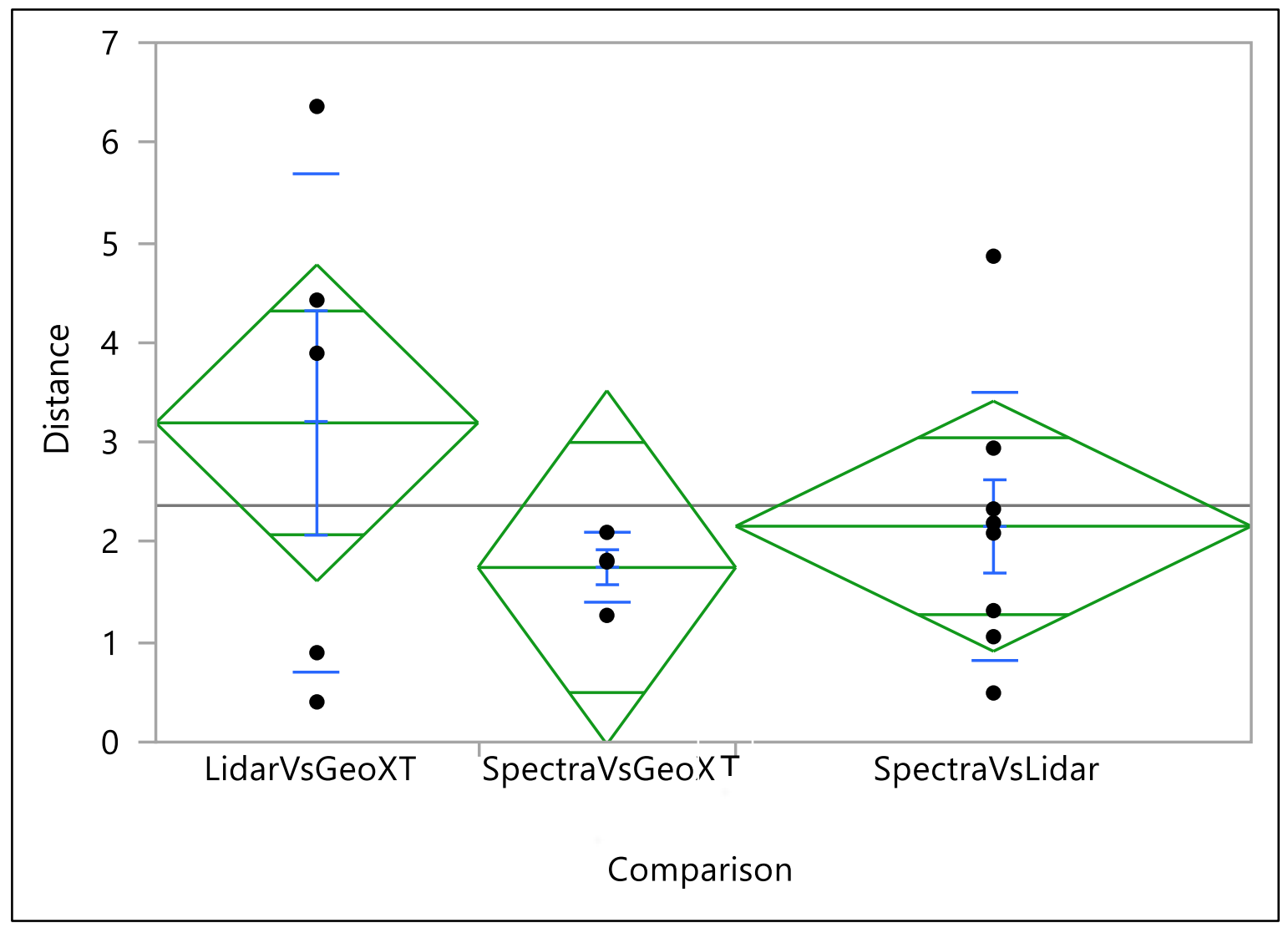

Figure 45: Pole Location Differences

The Using the least square means of pole location differences, we were able to determine that the Spectra and Trimble had a mean difference of $1.7147 \mathrm{~m}$ in pole locations, while the lidar differed from the Spectra by $2.1606 \mathrm{~m}$ and the Trimble by $3.1946 \mathrm{~m}$. Additionally, comparing 
the Spectra to the Trimble GeoXT reveals that the two GPS measures are more similar to each other than either was to the lidar. This seems to suggest that the tool being used for validation of tree location (GeoXT) is more accurate than the object being validated (lidar), which was a concern since the Spectra was not available for acquisition of tree base locations. Furthermore, as rather obvious location features, the pole locations serve as a measure of the $\mathrm{x}, \mathrm{y}$ or spatial accuracy of the point cloud. Thus, we determined that there is roughly $2.1606 \mathrm{~m}$ of position error inherent in our point cloud.

\section{Individual Tree Crown Detection and Segmentation}

In total, 72 models were generated from the combination of two DEMs for normalization (KNN and TIN), two point cloud densities (full and decimated), two unique canopy models (single and divided), three matching features (treetops and the two base approximation methods, $\mathrm{XY}$ and CP), and three matching routines (2.3 $\mathrm{m}$ from Pirotti 2010, our proposed $4.5 \mathrm{~m}$ method, and the tree height adaptive method from Esyn et al. 2015).

"Detection" was based upon tree count from within the five validation areas, where the number of trees from validation $(\mathrm{N}=172)$ is used as the denominator and the number of trees segmented from lidar in those areas is used as the numerator, and the resulting percentage is the percent detection. Tree matching routines allow the user to specifically say that a given tree in the lidar corresponds to a given tree in the field. Trees were considered "matched" when the difference of lidar and field measured heights were within $+/-15 \%$ and the estimated location of the lidar-derived tree and the validation tree were within a defined distance of each other. Our height matching threshold was determined by the accuracy limitations of the Nikon Forestry Pro 
handheld laser, which is $+/-15 \%$ of the total tree height. While our matching distance was dependent upon which of the three matching methods were utilized.

The three different matching methods in this study differed in the distance allowed between a validation tree and its matching lidar counterpart. We utilized the $2.3 \mathrm{~m}$ distance from Pirotti et al. (2010), a $4.5 \mathrm{~m}$ distance as a lenient measure, calculated as $2.3 \mathrm{~m}+\sim 2.2 \mathrm{~m}$ from position error of point cloud, and a tree height adaptive distance which is calculated as $10 \%$ of the tree's lidar derived height (Z), from Esyn et al. (2015). We compared models both across and within each matching method because as the matching distance becomes smaller, it restricts the amount of possible position error within matched trees at the expense of the model's matching rate. In total, our models created 1,417 matches of lidar derived trees and field trees.

Figure 46 displays the detection and matching rates of our models. Models that have divided the point cloud based upon vegetation canopy type, generally over-segmented the canopy in closed -canopy areas. Thus, these models obtained a detection rate $>100 \%$. In contrast, models that did not divide the point cloud based upon canopy type, generally under-segmented the closed -canopy areas.

models that used two different canopy types also had higher matching rates. Yet, matching rates were poor across all methods and all matching routines in the study. Furthermore, the matching feature "Tops", corresponding to the highest detected point of a given tree, was generally the best matching feature, which is somewhat counterintuitive considering its matching with the field tree's base location. The treetops matching feature performed best in precision, recall, F-score, and commission error, with only a few exceptions limited to the $2.3 \mathrm{~m}$ matching regiment, see Figure 47. The XY method, the mean $\mathrm{x}$ and mean $\mathrm{y}$ of all points within a given 
treeID, and the CP method, the center point of a given tree's crown polygon, were both outperformed by the treetops matching feature.

Furthermore, Esyn et al. (2015) suggested the index of match rate divided by the commission error rate to assess model performance. Thus, higher match rates are rewarded, while high commission error rates are penalized. Again, within this metric, methods utilizing the treetops matching feature performed best. The results of Esyn et al.'s (2015) index is available in Figure 48.

While "tops" excelled within the matching routines, they were excluded from the analysis of tree base location since a treetop (highest point) is not always at the same x,y location of its base. These estimated base locations will be used to determine if a tree can come into contact with electrical conductors in the Risk Analysis section. The RMSE of tree locations are provided in Figure 49. Table 5 includes precision, recall, F-score, omission error rate, commission error rate, Kappa, detection percentage, match percentage and Esyn et al.'s (2015) index. Notice, in Figure 49, that all Kappa values for the matching routine were negative due to extremely low matching rates. 




Figure 46: Tree Detection and Matching Rates by matching method and matching feature

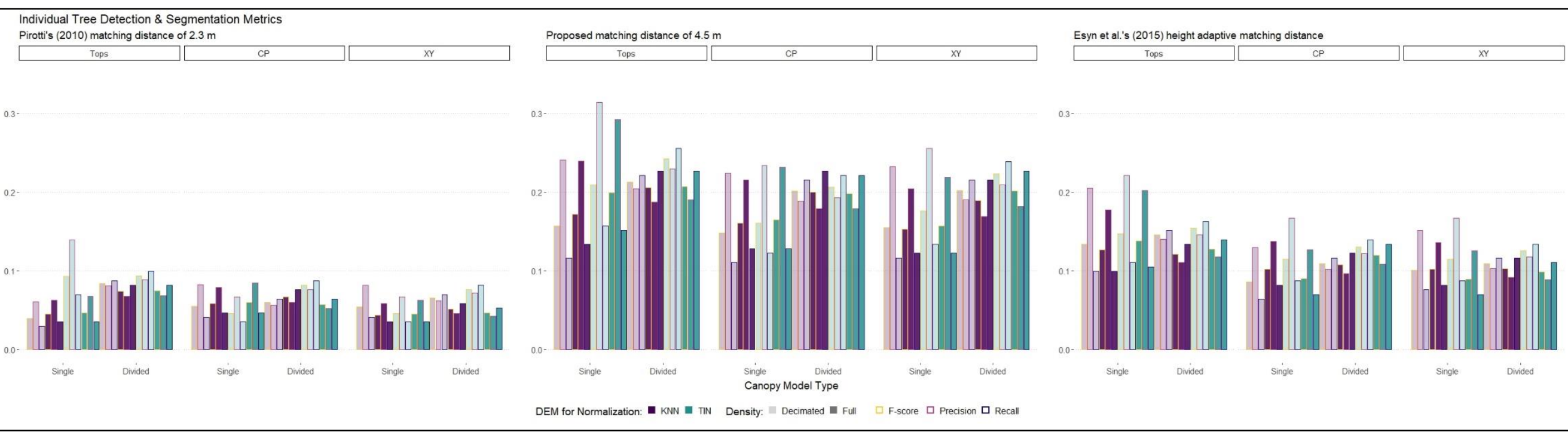

Figure 47: F-score, Precision, and Recall by matching method and matching feature 


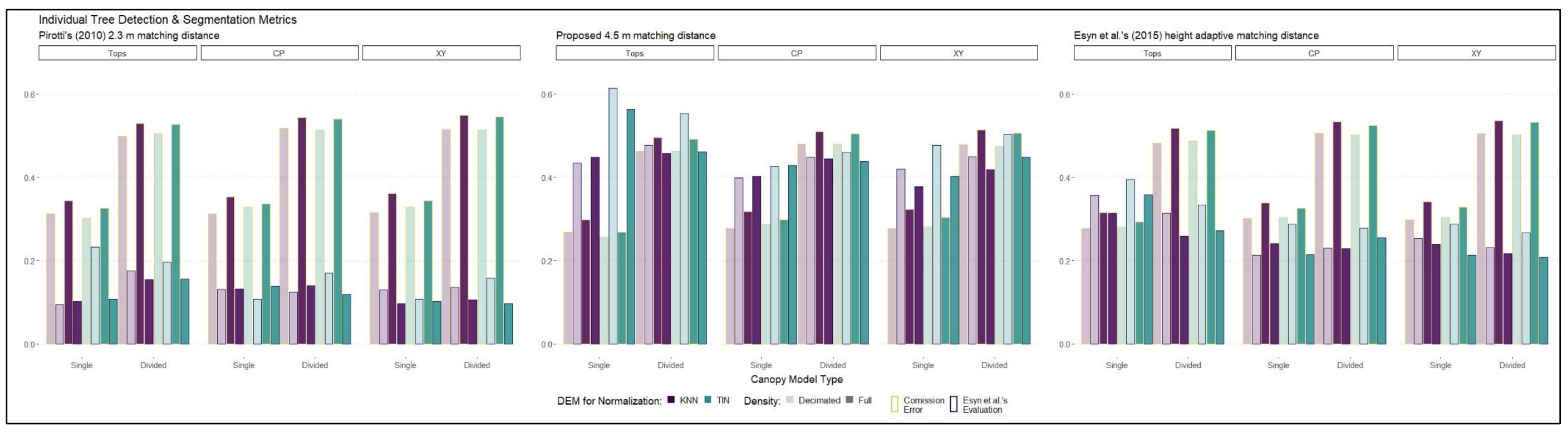

Figure 48: Commission Error Rate and Esyn et al.'s (2015) accuracy index by matching method and matching feature 




Figure 49: Kappa of Agreement for Matching and Tree Location RMSE by matching method and matching feature 
Table 5: Individual Tree Detection and Segmentation accuracy metrics for all models

\begin{tabular}{|c|c|c|c|c|c|c|c|c|c|c|c|c|c|c|c|c|}
\hline \multirow{31}{*}{1} & \multirow{16}{*}{ KNN } & \multirow{8}{*}{ Decimated } & $\mathrm{CP}$ & $4.5 \mathrm{~m}$ & \multirow{8}{*}{452} & \multirow{2}{*}{85} & 19 & 0.2235 & 0.1105 & 0.1479 & 0.2773 & 0.6429 & -0.7448 & $49 \%$ & $11 \%$ & 0.3983 \\
\hline & & & & Adaptive & & & 11 & 0.1294 & 0.0640 & 0.0856 & 0.3008 & 0.6545 & -0.9453 & $49 \%$ & $6 \%$ & 0.2126 \\
\hline & & & \multirow{3}{*}{ Tops } & $2.3 \mathrm{~m}$ & & & 5 & 0.0602 & 0.0291 & 0.0392 & 0.3120 & 0.6680 & -1.1272 & $48 \%$ & $3 \%$ & 0.0932 \\
\hline & & & & $4.5 \mathrm{~m}$ & & 83 & 20 & 0.2410 & 0.1163 & 0.1569 & 0.2681 & 0.6468 & -0.7336 & $48 \%$ & $12 \%$ & 0.4337 \\
\hline & & & & Adaptive & & & 17 & 0.2048 & 0.0988 & 0.1333 & 0.2773 & 0.6513 & -0.8061 & $48 \%$ & $10 \%$ & 0.3564 \\
\hline & & & & $2.3 \mathrm{~m}$ & & & 7 & 0.0814 & 0.0407 & 0.0543 & 0.3147 & 0.6574 & -1.0463 & $50 \%$ & $4 \%$ & 0.1293 \\
\hline & & & $X Y$ & $4.5 \mathrm{~m}$ & & 86 & 20 & 0.2326 & 0.1163 & 0.1550 & 0.2773 & 0.6387 & -0.7152 & $50 \%$ & $12 \%$ & 0.4193 \\
\hline & & & & Adaptive & & & 13 & 0.1512 & 0.0756 & 0.1008 & 0.2980 & 0.6490 & -0.8864 & $50 \%$ & $8 \%$ & 0.2537 \\
\hline & & & & $2.3 \mathrm{~m}$ & & & 8 & 0.0784 & 0.0465 & 0.0584 & 0.3534 & 0.6165 & -0.9263 & $59 \%$ & $5 \%$ & 0.1316 \\
\hline & & & CP & $4.5 \mathrm{~m}$ & & 102 & 22 & 0.2157 & 0.1279 & 0.1606 & 0.3175 & 0.5952 & -0.6004 & $59 \%$ & $13 \%$ & 0.4029 \\
\hline & & & & Adaptive & & & 14 & 0.1373 & 0.0814 & 0.1022 & 0.3385 & 0.6077 & -0.7797 & $59 \%$ & $8 \%$ & 0.2405 \\
\hline & & & & $2.3 \mathrm{~m}$ & & & 6 & 0.0625 & 0.0349 & 0.0448 & 0.3435 & 0.6336 & -1.0083 & $56 \%$ & $3 \%$ & 0.1016 \\
\hline & & Full & Tops & $4.5 \mathrm{~m}$ & 514 & 96 & 23 & 0.2396 & 0.1337 & 0.1716 & 0.2980 & 0.6082 & -0.6002 & $56 \%$ & $13 \%$ & 0.4488 \\
\hline & & & & Adaptive & & & 17 & 0.1771 & 0.0988 & 0.1269 & 0.3147 & 0.6175 & -0.7344 & $56 \%$ & $10 \%$ & 0.3140 \\
\hline & & & $\mathrm{XY}$ & $4.5 \mathrm{~m}$ & & 103 & 21 & 0.2039 & 0.1221 & 0.1527 & 0.3228 & 0.5945 & -0.6187 & $60 \%$ & $12 \%$ & 0.3782 \\
\hline & & & & Adaptive & & & 14 & 0.1359 & 0.0814 & 0.1018 & 0.3410 & 0.6054 & -0.7759 & $60 \%$ & $8 \%$ & 0.2387 \\
\hline & & & & $2.3 \mathrm{~m}$ & & & 6 & 0.0667 & 0.0349 & 0.0458 & 0.3281 & 0.6484 & -1.0453 & $52 \%$ & $3 \%$ & 0.1063 \\
\hline & & & CP & $4.5 \mathrm{~m}$ & & 90 & 21 & 0.2333 & 0.1221 & 0.1603 & 0.2863 & 0.6266 & -0.6708 & $52 \%$ & $12 \%$ & 0.4264 \\
\hline & & & & Adaptive & & & 15 & 0.1667 & 0.0872 & 0.1145 & 0.3036 & 0.6356 & -0.8119 & $52 \%$ & $9 \%$ & 0.2872 \\
\hline & & & & $2.3 \mathrm{~m}$ & & & 12 & 0.1395 & 0.0698 & 0.0930 & 0.3008 & 0.6504 & -0.9122 & $50 \%$ & $7 \%$ & 0.2319 \\
\hline & & Decimated & Tops & $4.5 \mathrm{~m}$ & 460 & 86 & 27 & 0.3140 & 0.1570 & 0.2093 & 0.2554 & 0.6277 & $\begin{array}{l}-0.5590 \\
0.727\end{array}$ & $50 \%$ & $16 \%$ & 0.6146 \\
\hline & & & & Adaptive & & & 19 & 0.2209 & 0.1105 & 0.1473 & 0.2803 & 0.6402 & -0.7387 & $50 \%$ & $11 \%$ & 0.3940 \\
\hline & & & & $2.3 \mathrm{~m}$ & & & 6 & 0.0667 & 0.0349 & 0.0458 & 0.3281 & 0.6484 & -1.0453 & $52 \%$ & $3 \%$ & 0.1063 \\
\hline & & & $\mathrm{XY}$ & $4.5 \mathrm{~m}$ & & 90 & 23 & 0.2556 & 0.1337 & 0.1756 & 0.2803 & 0.6234 & -0.6261 & $52 \%$ & $13 \%$ & 0.4770 \\
\hline & TIN & & & Adaptive & & & 15 & 0.1667 & 0.0872 & 0.1145 & 0.3036 & 0.6356 & -0.8119 & $52 \%$ & $9 \%$ & 0.2872 \\
\hline & niv & & & $2.3 \mathrm{~m}$ & & & 8 & 0.0842 & 0.0465 & 0.0599 & 0.3359 & 0.6332 & -0.9610 & $55 \%$ & $5 \%$ & 0.1385 \\
\hline & & & CP & $4.5 \mathrm{~m}$ & & 95 & 22 & 0.2316 & 0.1279 & 0.1648 & 0.2980 & 0.6122 & -0.6259 & $55 \%$ & $13 \%$ & 0.4293 \\
\hline & & & & Adaptive & & & 12 & 0.1263 & 0.0698 & 0.0899 & 0.3255 & 0.6275 & -0.8591 & $55 \%$ & $7 \%$ & 0.2143 \\
\hline & & & & $\begin{array}{l}\text { Adaptive } \\
2.3 \mathrm{~m}\end{array}$ & & & $\begin{array}{c}18 \\
6\end{array}$ & $\begin{array}{l}0.2022 \\
0.0625\end{array}$ & $\begin{array}{l}0.1047 \\
0.0349\end{array}$ & $\begin{array}{l}0.1379 \\
0.0448\end{array}$ & $\begin{array}{l}0.2922 \\
0.3435\end{array}$ & $\begin{array}{l}0.6337 \\
0.6336\end{array}$ & $\begin{array}{l}-0.7453 \\
-1.0083\end{array}$ & $\begin{array}{l}52 \% \\
56 \%\end{array}$ & $\begin{array}{l}10 \% \\
3 \%\end{array}$ & $\begin{array}{l}0.3582 \\
0.1016\end{array}$ \\
\hline & & & $\mathrm{XY}$ & $\begin{array}{l}2.3 \mathrm{~m} \\
4.5 \mathrm{~m}\end{array}$ & & 96 & 21 & 0.2188 & 0.1221 & $\begin{array}{l}0.04467 \\
0.1567\end{array}$ & 0.3035 & 0.6113 & $\begin{array}{l}-1.06438 \\
-0.6438\end{array}$ & $56 \%$ & $12 \%$ & 0.4021 \\
\hline & & & & Adaptive & & 00 & 12 & 0.1250 & 0.0698 & 0.0896 & 0.3281 & 0.6250 & $\begin{array}{l}-0.08541 \\
-0.854\end{array}$ & $56 \%$ & $7 \%$ & 0.2126 \\
\hline & & & & $2.3 \mathrm{~m}$ & & & 11 & 0.0561 & 0.0640 & 0.0598 & 0.5182 & 0.4510 & -0.7726 & $114 \%$ & $6 \%$ & 0.1234 \\
\hline & & & CP & $4.5 \mathrm{~m}$ & & 196 & 37 & 0.1888 & 0.2151 & 0.2011 & 0.4804 & 0.4079 & -0.3018 & $114 \%$ & $22 \%$ & 0.4478 \\
\hline & & & & Adaptive & & & 20 & 0.1020 & 0.1163 & 0.1087 & 0.5057 & 0.4368 & -0.5968 & $114 \%$ & $12 \%$ & 0.2299 \\
\hline & & & & $2.3 \mathrm{~m}$ & & & 15 & 0.0806 & 0.0872 & 0.0838 & 0.4985 & 0.4577 & -0.6843 & $108 \%$ & $9 \%$ & 0.1749 \\
\hline & & Decimated & Tops & $4.5 \mathrm{~m}$ & 1100 & 186 & 38 & 0.2043 & 0.2209 & 0.2123 & 0.4625 & 0.4188 & -0.2756 & $108 \%$ & $22 \%$ & 0.4777 \\
\hline & & & & Adaptive & & & 26 & 0.1398 & 0.1512 & 0.1453 & 0.4819 & 0.4398 & -0.4779 & $108 \%$ & $15 \%$ & 0.3137 \\
\hline & & & & $2.3 \mathrm{~m}$ & & & 12 & 0.0615 & 0.0698 & 0.0654 & 0.5155 & 0.4507 & -0.7514 & $113 \%$ & $7 \%$ & 0.1353 \\
\hline & & & $\mathrm{XY}$ & $4.5 \mathrm{~m}$ & & 195 & 37 & 0.1897 & 0.2151 & 0.2016 & 0.4788 & 0.4091 & -0.3008 & $113 \%$ & $22 \%$ & 0.4493 \\
\hline & KNN & & & Adaptive & & & 20 & 0.1026 & 0.1163 & 0.1090 & 0.5043 & 0.4380 & -0.5958 & $113 \%$ & $12 \%$ & 0.2306 \\
\hline & & & & $2.3 \mathrm{~m}$ & & & 13 & 0.0596 & 0.0756 & 0.0667 & 0.5438 & 0.4218 & -0.7560 & $127 \%$ & $8 \%$ & 0.1390 \\
\hline & & & CP & $4.5 \mathrm{~m}$ & & 218 & 39 & 0.1789 & 0.2267 & 0.2000 & 0.5100 & 0.3789 & -0.2966 & $127 \%$ & $23 \%$ & 0.4446 \\
\hline & & & & Adaptive & & & 21 & 0.0963 & 0.1221 & 0.1077 & 0.5339 & 0.4092 & -0.6034 & $127 \%$ & $12 \%$ & 0.2287 \\
\hline & & & & $2.3 \mathrm{~m}$ & & & 14 & 0.0673 & 0.0814 & 0.0737 & 0.5301 & 0.4317 & $\begin{array}{l}-0.7245 \\
\end{array}$ & $121 \%$ & $8 \%$ & 0.1536 \\
\hline & & Full & Tops & $4.5 \mathrm{~m}$ & 1152 & 208 & 39 & 0.1875 & 0.2267 & 0.2053 & 0.4956 & 0.3900 & -0.2839 & $121 \%$ & $23 \%$ & 0.4575 \\
\hline & & & & Adaptive & & & 23 & 0.1106 & 0.1337 & 0.1211 & 0.5182 & 0.4174 & -0.5545 & $121 \%$ & $13 \%$ & 0.2580 \\
\hline & & & & $2.3 \mathrm{~m}$ & & & 10 & 0.0457 & 0.0581 & 0.0512 & 0.5486 & 0.4252 & -0.8172 & $127 \%$ & $6 \%$ & 0.1060 \\
\hline & & & $\mathrm{XY}$ & $\begin{array}{c}4.5 \mathrm{~m} \\
\text { Adaptive }\end{array}$ & & 219 & 37 & 0.1689 & 0.2151 & 0.1893 & 0.5141 & 0.3814 & $\begin{array}{l}-0.3298 \\
-06232\end{array}$ & $127 \%$ & $22 \%$ & 0.4184 \\
\hline 2 & & & & $\begin{array}{l}\text { Adaptive } \\
2.3 \mathrm{~m}\end{array}$ & & & 20 & 0.0913 & 0.1163 & 0.1023 & 0.5364 & 0.4097 & -0.6232 & $127 \%$ & $12 \%$ & 0.2168 \\
\hline & & & CP & $\begin{array}{l}2.3 \mathrm{~m} \\
4.5 \mathrm{~m}\end{array}$ & & 197 & $\begin{array}{l}15 \\
38\end{array}$ & 0.0761 & 0.0872 & 0.0813 & 0.5141 & 0.4435 & $\begin{array}{l}-0.6935 \\
-02899\end{array}$ & $115 \%$ & $9 \%$ & 0.1696 \\
\hline & & & CP & $\begin{array}{l}4.5 \mathrm{~m} \\
\text { Adaptive }\end{array}$ & & 197 & $\begin{array}{l}38 \\
24\end{array}$ & $\begin{array}{l}0.1929 \\
0.1218\end{array}$ & $\begin{array}{l}0.2209 \\
0.1395\end{array}$ & $\begin{array}{l}0.2060 \\
0.1301\end{array}$ & $\begin{array}{l}0.4804 \\
0.5014\end{array}$ & $\begin{array}{l}0.4448 \\
0.0290\end{array}$ & $\begin{array}{r}-0.2869 \\
-0.5242\end{array}$ & $\begin{array}{l}115 \% \\
115 \%\end{array}$ & $\begin{array}{l}22 \% \\
14 \%\end{array}$ & 0.4599 \\
\hline & & & & $2.3 \mathrm{~m}$ & & & 17 & 0.0885 & 0.0988 & 0.0934 & 0.5043 & 0.4467 & -0.6501 & $112 \%$ & $10 \%$ & 0.1960 \\
\hline & & Decimated & Tops & $4.5 \mathrm{~m}$ & 1091 & 192 & 44 & 0.2292 & 0.2558 & 0.2418 & 0.4625 & 0.4000 & -0.1886 & $112 \%$ & $26 \%$ & 0.5531 \\
\hline & & & & Adaptive & & & 28 & 0.1458 & 0.1628 & 0.1538 & 0.4881 & 0.4286 & -0.4482 & $112 \%$ & $16 \%$ & 0.3335 \\
\hline & & & & $2.3 \mathrm{~m}$ & & & 14 & 0.0714 & 0.0814 & 0.0761 & 0.5141 & 0.4463 & -0.7123 & $114 \%$ & $8 \%$ & 0.1583 \\
\hline & & & $\mathrm{XY}$ & $4.5 \mathrm{~m}$ & & 196 & 41 & 0.2092 & 0.2384 & 0.2228 & 0.4740 & 0.4006 & -0.2388 & $114 \%$ & $24 \%$ & 0.5029 \\
\hline & & & & Adaptive & & & 23 & 0.1173 & 0.1337 & 0.1250 & 0.5014 & 0.4319 & -0.5414 & $114 \%$ & $13 \%$ & 0.2667 \\
\hline & TIN & & & $2.3 \mathrm{~m}$ & & & 11 & 0.0516 & 0.0640 & 0.0571 & 0.5401 & 0.4305 & -0.7899 & $124 \%$ & $6 \%$ & 0.1184 \\
\hline & & & $\mathrm{CP}$ & $4.5 \mathrm{~m}$ & & 213 & 38 & 0.1784 & 0.2209 & 0.1974 & 0.5043 & 0.3862 & -0.3060 & $124 \%$ & $22 \%$ & 0.4381 \\
\hline & & & & Adaptive & & & 23 & 0.1080 & 0.1337 & 0.1195 & 0.5249 & 0.4116 & -0.5606 & $124 \%$ & $13 \%$ & 0.2548 \\
\hline & & & & $2.3 \mathrm{~m}$ & & & 14 & 0.0683 & 0.0814 & 0.0743 & 0.5262 & 0.4353 & -0.7212 & $119 \%$ & $8 \%$ & 0.1547 \\
\hline & & Full & Tops & $4.5 \mathrm{~m}$ & 1136 & 205 & 39 & 0.1902 & 0.2267 & 0.2069 & 0.4911 & 0.3935 & -0.2803 & $119 \%$ & $23 \%$ & 0.4617 \\
\hline & & & & Adaptive & & & 24 & 0.1171 & 0.1395 & 0.1273 & 0.5127 & 0.4193 & -0.5330 & $119 \%$ & $14 \%$ & 0.2721 \\
\hline & & & & $2.3 \mathrm{~m}$ & & & 9 & 0.0419 & 0.0523 & 0.0465 & 0.5450 & 0.4312 & -0.8328 & $125 \%$ & $5 \%$ & 0.0960 \\
\hline & & & $\mathrm{XY}$ & $4.5 \mathrm{~m}$ & & 215 & 39 & 0.1814 & 0.2267 & 0.2016 & 0.5057 & 0.3822 & -0.2927 & $125 \%$ & $23 \%$ & 0.4483 \\
\hline & & & & Adaptive & & & 19 & 0.0884 & 0.1105 & 0.0982 & 0.5326 & 0.4158 & -0.6368 & $125 \%$ & $11 \%$ & 0.2074 \\
\hline
\end{tabular}


Since the primary goal of the study was the detection, matching, and risk assessment of NERC category $2 \& 3$ trees, we further investigate detection and segmentation results within each matching method. The following sections will continue to explore the accuracy of the measurement of matched trees' physical properties from within UAS-lidar point cloud. First, the differences in the elevation at the estimated base locations will be explored, before lidar -derived tree height $(\mathrm{Z})$ and the predicted DBH. After which, we detail the results from our tree risk analysis where, the results from our classification of Threat Detection, Likelihood of Failure, Likelihood of Impact, Consequences of Impact, and Final Risk Rating are analyzed, both within each matching routine and across all matching routines.

\section{Tree Base Location}

Trees were considered "matched" when the difference of lidar and field measured heights were within $+/-15 \%$ and the validation base location and the lidar estimated location of the tree were within the matching routine's specified distance from each other. The distance between the Trimble GeoXT field measured (validation) base and the lidar predicted tree base matching feature (base $\mathrm{CP}$, base $\mathrm{XY}$ ) was subjected to ANOVA to discern differences in tree base locations due to the model parameters: singular or dual models for canopy type, DEM for normalization, point cloud density, and the matching feature. Additionally, as this is a question of tree base, models using the treetops matching feature were excluded and thus, $\mathrm{N}=48$ models here.

The ANOVA for the distance between matched tree's approximated base locations within the $2.3 \mathrm{~m}$ matching method revealed a statistically significant difference in the tree base location of matched trees $(\mathrm{p}$-value $=0.0465)$. The effect tests determined that the difference was due to the density parameter $(\mathrm{p}$-value $=0.0131)$, where the full density models possessed a mean 
difference of $1.3577 \mathrm{~m}$ in matched tree base locations, while the decimated models possessed a mean difference of $1.5847 \mathrm{~m}$. Thus, this suggests that the full density models are $\sim 0.2 \mathrm{~m}$ more accurate at determining tree base location, when using a matching distance of $2.3 \mathrm{~m}$. In contrast, the ANOVAs on the distance between matched trees' approximated base locations within $4.5 \mathrm{~m}$ and adaptive matching routines found no statistically significant differences in the locations of matched trees' base locations ( $\mathrm{p}$-value $=0.8585$ and 0.5601 , respectively). RMSE of Tree Location is summarized in Figure 49.

\section{Tree Base Elevation}

Initially, we had intended to compare our lidar derived tree base elevations with field acquired tree base elevations from the Spectra SP80. When we were not able to obtain the Spectra for subsequent field visits, we decided to compare our lidar derived tree base elevations to the corresponding DEM elevation values at the locations of our field acquired tree base GPS points. This was completed such that TIN normalized lidar models were compared to the TIN elevations at our field data's tree base locations and KNN normalized lidar models were compared to the KNN elevations at our field data's tree base locations. This was possible using ArcGIS PRO's "extract values to points" to extract the TIN and KNN DEM values to the field tree's base location points shapefile. Additionally, as this is a question of tree base, models using the treetops matching feature were excluded and thus, $\mathrm{N}=48$ models here.

The overall ANOVA of the $2.3 \mathrm{~m}$ matching method's difference of validation tree base elevation and lidar derived tree base elevation at matched tree bases was not significant, ( $p$-value $=0.2187)$. Yet, the effect tests identified that the DEM used for point cloud normalization has a statistically significant effect on the differences of elevation at matched tree bases ( $\mathrm{p}$-value $=$ 0.0384). The average difference of validation and KNN normalized tree base elevations was 
$0.2765 \mathrm{~m}$ and the average difference of validation and TIN normalized tree base elevations was $0.0453 \mathrm{~m}$. Thus, suggesting that TIN DEM normalized models are better at predicting a tree's base elevation within the $2.3 \mathrm{~m}$ matching method. Additionally, given that values for both TIN and KNN are positive, the results suggest that the lidar has underestimated the elevation at tree base locations of matched trees within the 2.3 m matching method.

ANOVAs of the $4.5 \mathrm{~m}$ and adaptive matching methods' differences of field tree base elevation and lidar derived matched tree bases were not signficant ( $\mathrm{p}$-value $=0.1378 \& 0.0681$ ). Yet, the effect tests for the adaptive method identify that the DEM used for normalization demonstrated a statistically significant difference in the mean differences of elevation at matched tree bases $(\mathrm{p}$-value $=0.0099)$. The average difference of field tree base elevations and KNN normalized tree base elevations was $0.1773 \mathrm{~m}$ and the average difference of field tree base elevations and TIN normalized tree base elevations was $-0.0971 \mathrm{~m}$. Thus, these results indicate once again that TIN DEM normalized models were better at predicting a tree's base elevation. Yet, note that within the adaptive matching method, KNN models appear to underestimate the elevation at matched trees bases, while the TIN models appear to overestimate the elevation at matched trees bases.

\section{Tree Height}

Lidar derived tree heights $(Z)$ were fitted against their matched field trees' heights, and the resulting measures of R-square, correlation, and RMSE are provided in Figure 50. The correlation values of tree height for the $2.3 \mathrm{~m}$ matching routine ranged from 0.9158 to 0.9934 . All correlations were found to be significant, with $\mathrm{p}$-values for correlation ranging from $<0.0001$ to 0.0081 . RMSE ranged from $2.512 \mathrm{~m}$ to $0.7970 \mathrm{~m}$, with an average of $1.6554 \mathrm{~m}$. R-square values ranged from 0.8388 to 0.9869 , with a mean of 0.9380 . The differences of validation tree 
height and lidar derived tree height were calculated and the overall ANOVA on the differences was significant $(\mathrm{p}$-value $=0.0328)$. The effect tests detected that the vegetation canopy modeling parameter had a statistically significant impact on the differences of matched field measured and lidar derived tree heights $(\mathrm{p}$-value $=0.0015)$. The divided canopy models were more accurate with a mean difference of tree height of $-0.1301 \mathrm{~m}$, compared to the single canopy models, which had a mean difference of tree height of $-0.9292 \mathrm{~m}$.

Within the $4.5 \mathrm{~m}$ matching method, correlation values ranged from 0.9361 to 0.9819 and all correlations were found to be statistically significant (p-values: $<0.0001$ ). RMSE ranged from $1.3772 \mathrm{~m}$ to $2.0555 \mathrm{~m}$, with an average of $1.7674 \mathrm{~m}$. R-square values ranged from 0.8762 to 0.9237, with a mean of 0.9293 . Meanwhile, correlation values for the adaptive matching routine ranged from 0.8535 to 0.9441 , all correlations were found to be statistically significant (p-values: $<0.0001)$. The RMSE ranged from $1.3944 \mathrm{~m}$ to $2.3231 \mathrm{~m}$, with an average of $1.904 \mathrm{~m}$. R-square values ranged from 0.7285 to 0.8914 , with a mean of 0.8263 . The differences of field trees heights and lidar derived trees heights were calculated for both the $4.5 \mathrm{~m}$ and adaptive matching methods and, the ANOVAs of the differences were not significant (p-values: 0.4996 and 0.3749 , respectively). 




Figure 50: Matched Tree Height R-square, correlation, and RMSE by matching method and matching feature 


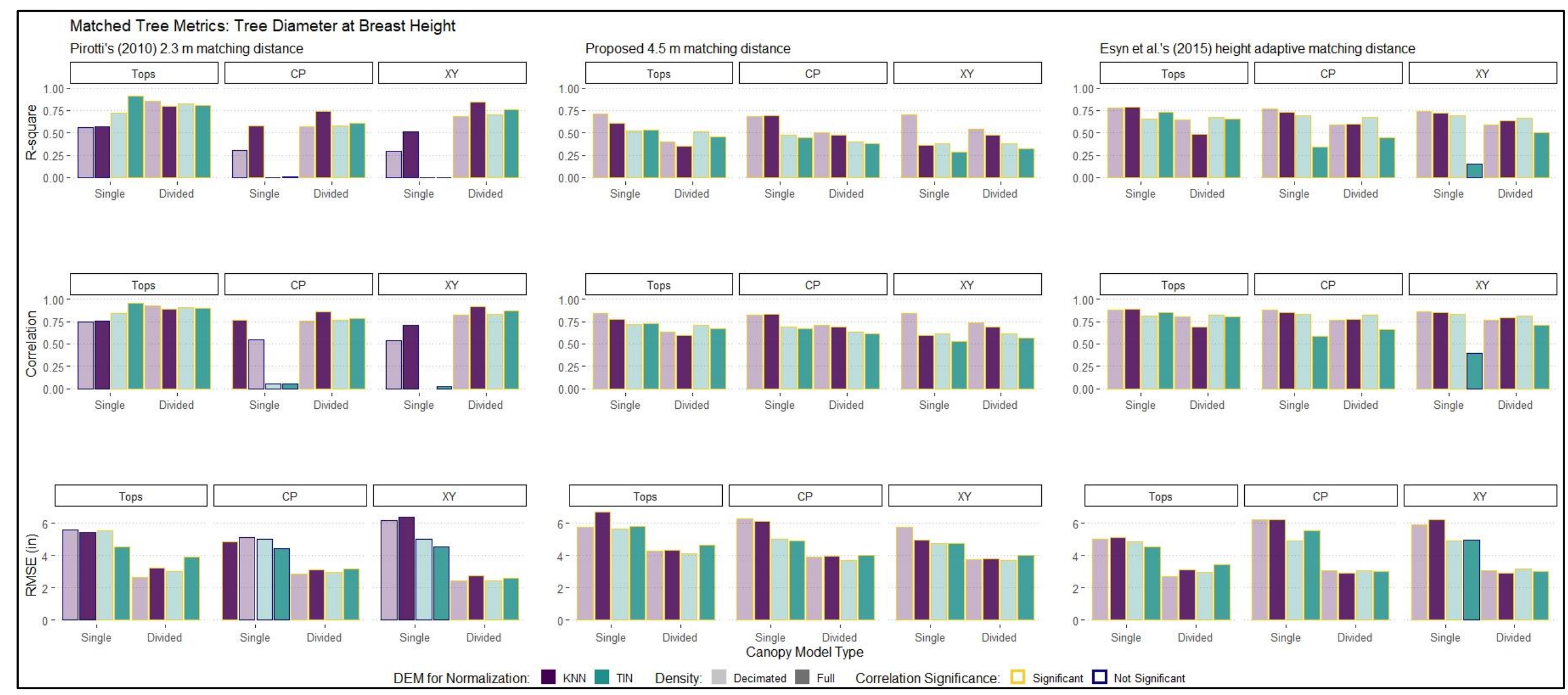

Figure 51: Matched Tree DBH R-square, correlation, and RMSE by matching method and matching feature 


\section{Tree $\mathrm{DBH}$}

Matched trees' lidar predicted DBHs were fitted against their field measured DBH and, the measures of R-square, correlation, RMSE, and significance are provided in Figure 51. The difference of validation DBH and the lidar predicted DBH was then calculated and subjected to ANOVA.

Within the $2.3 \mathrm{~m}$ matching method correlation values ranged from -0.0014 to 0.9518 , with a median correlation of 0.7718 . The p-values of correlation ranged from $<0.0001$ to 0.9978 , with a median of 0.0039 . Thus, over half the models were strongly correlated and statistically significant. RMSE ranged from 2.7932 inches to 5.686 inches, with a mean of 4.0986 inches. Rsquare values ranged from $2.06^{\mathrm{e}-6}$ to 0.9059 , with a mean of 0.5495 . The ANOVA of the differences of field and lidar derived trees DBHs was not significant ( $p$ value $=0.2961$ ), and the effect tests only demonstrate a trend towards a difference due to the effect of vegetation canopy type $(\mathrm{p}$ value $=0.0516)$. The average difference in $\mathrm{DBH}($ field - predicted $)$ for the single canopy models was 2.1148 inches, while the divided canopy models had a difference of only 0.9366 inches.

The correlation values for the $4.5 \mathrm{~m}$ matching routine ranged from 0.5311 to 0.8444 , with a median correlation of 0.6871 . The p-values of correlation ranged from $<0.0001$ to 0.0132 , with a median of 0.00002 . Thus, all of the correlations of the lidar predicted diameter and field measured diameter were statistically significant. RMSE ranged from 3.6071 inches to 5.0884 inches, with a mean of 4.1973 inches and, R-square values ranged from 0.2820 to 0.7130 , with a mean of 0.4812. Yet, the ANOVA for the differences of field and lidar derived tree DBHs was not significant $(\mathrm{p}$ value $=0.8017)$. 
Lastly, the correlation values for the adaptive matching routine ranged from 0.3921 to 0.8879 , with a median correlation of 0.8093 . The $p$-values of correlation ranged from $<0.0001$ to 0.2074 , with a median of 0.00008 and, all but one of the models were statistically significant. RMSE ranged from 3.2226 inches to 4.4379 inches, with a mean of 3.832 inches. Likewise, Rsquare values ranged from 0.1537 to 0.7884 , with a mean of 0.6198 . The ANOVA of differences of field DBHs and lidar predicted DBHs were not significant $(\mathrm{p}$-value $=0.9966)$ and effect tests detected no differences.

\section{Risk Analysis}

This section summarizes the results from the contingency analyses of our Threat Detection and tree risk assessment classifications for Threat Detection, Likelihood of Failure, Likelihood of Impact, Consequences of Impact, and the Final Risk Rating. We described the agreement between our lidar and field classifications as Kappa agreement statistics. The probability $>\mathrm{z}$ associated with each Kappa value was used to determine significance. In the Likelihood of Failure classification, $\mathrm{N}=72$ since all models could calculate a Likelihood of Failure measure from tree height and predicted DBH. In all other classifications, $\mathrm{N}=48$ minus the number of models where Kappa could not be calculated due to the contingency table.

Due to the number of models in the study, Figures 52 and 53 display the Kappa values as distributions. the figure uses lines to represent each model's individual path across each classification. Also, in Figure 52, Kappa values deemed in significant by their probability > p are demarcated differently than significant Kappa values, and treetops models were excluded. A Kappa value of 1.0 demonstrates "perfect agreement" between the field and lidar derived datasets, whereas 0.0 indicates "no agreement". 
Risk Assessment Classification Agreement

Pirotti's (2010) $2.3 \mathrm{~m}$ matching distance

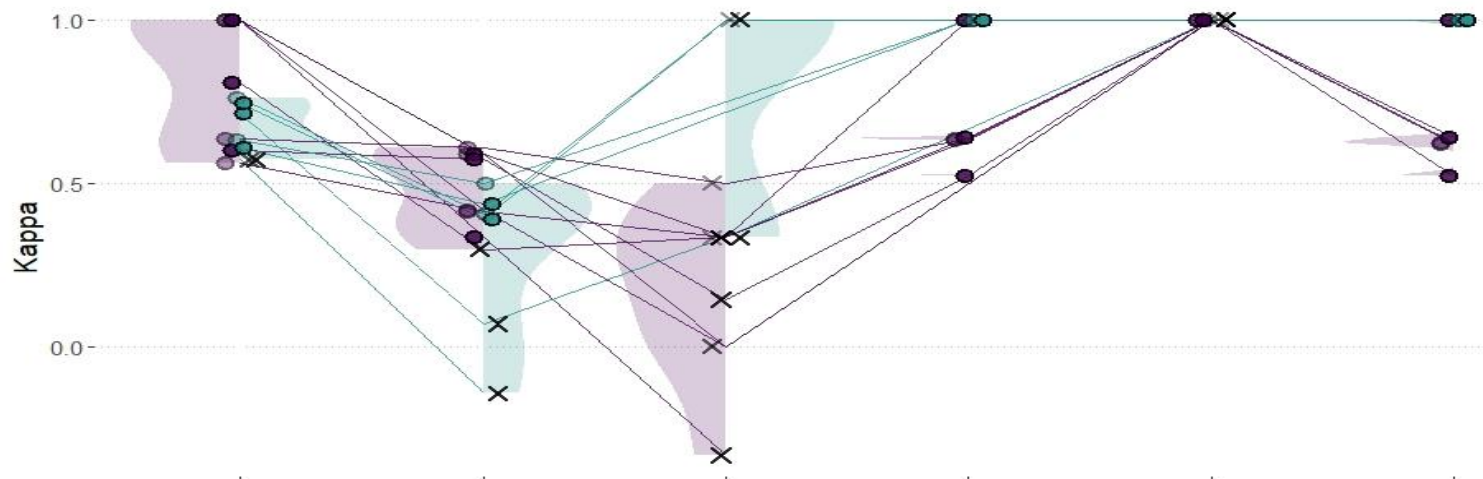

Threat Detection Likelihood of Failure Like lihood of Impact Likelihood Matrix Consequences of Impact Final Risk Rating

Proposed $4.5 \mathrm{~m}$ matching distance

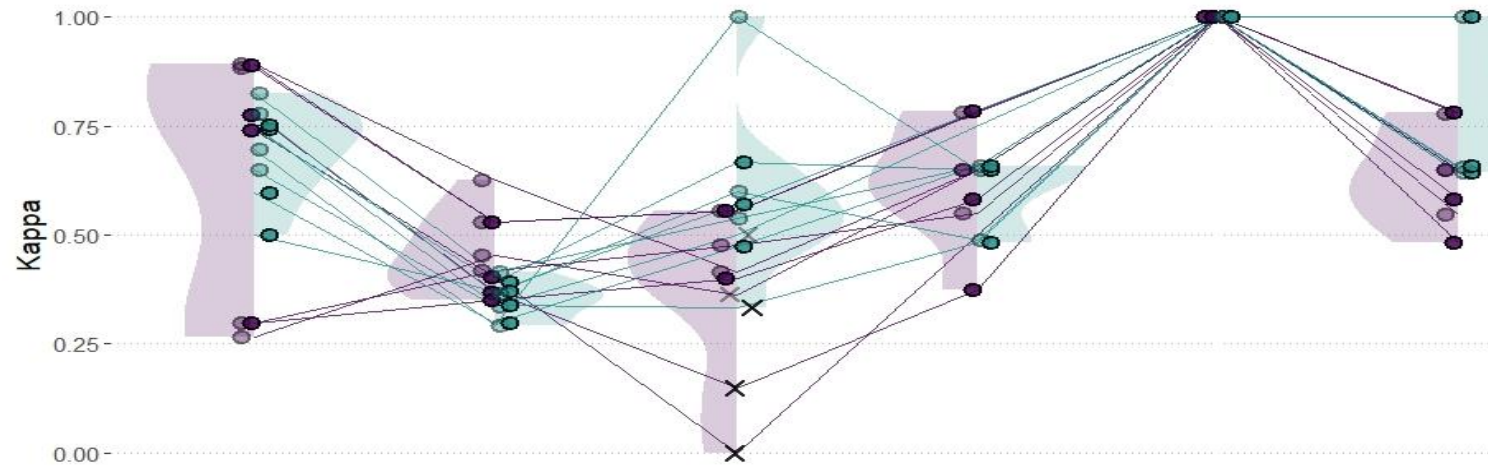

Threat Detection Likelihood of Failure Like lihood of Impact Likelihood Matrix Consequenoes of Impact Final Risk Rating

Esyn et al.'s (2015) height adaptive matching distance

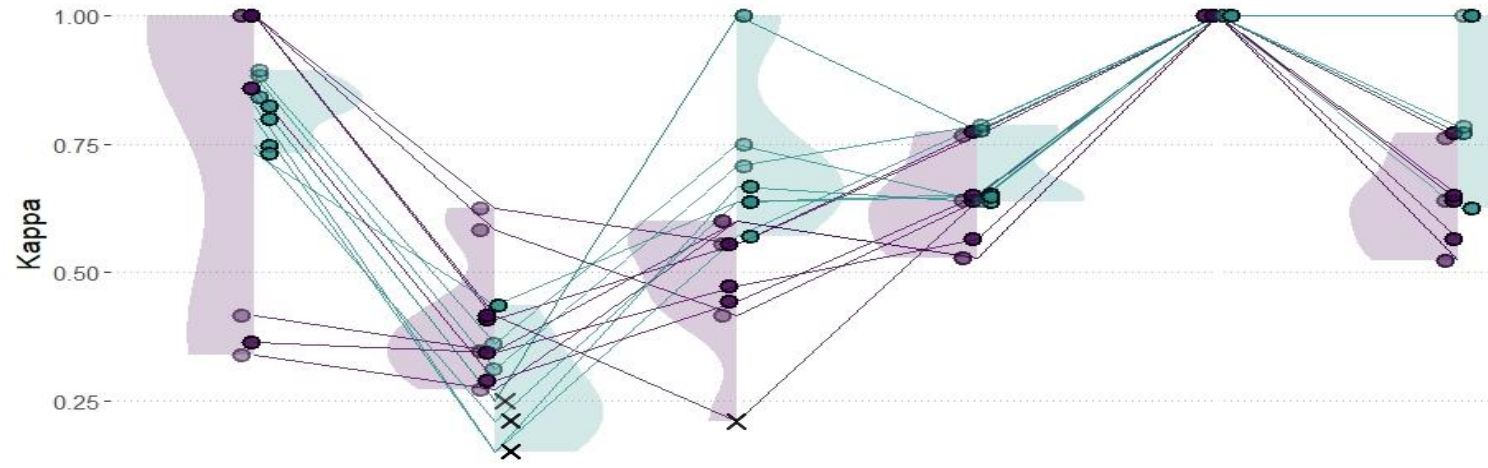

$$
\begin{aligned}
& \text { Threat Detection Likelihood of Failure Likelihood of Impact Likelihood Matrix consequenoes of Impact Final Risk Rating } \\
& \text { DEM for Normalization: }- \text { KNN } \square \text { TIN Density: O Decimated - Full Kappa Significance: O Significant } \times \text { Insignificant }
\end{aligned}
$$

Figure 52: Distributions of Kappa Agreement Statistics for each risk analysis classification by matching method 
Kappa Agreement Statistics for Risk Assessment Classification

Distribution of Kappa for the Likelihood of Failure Classification

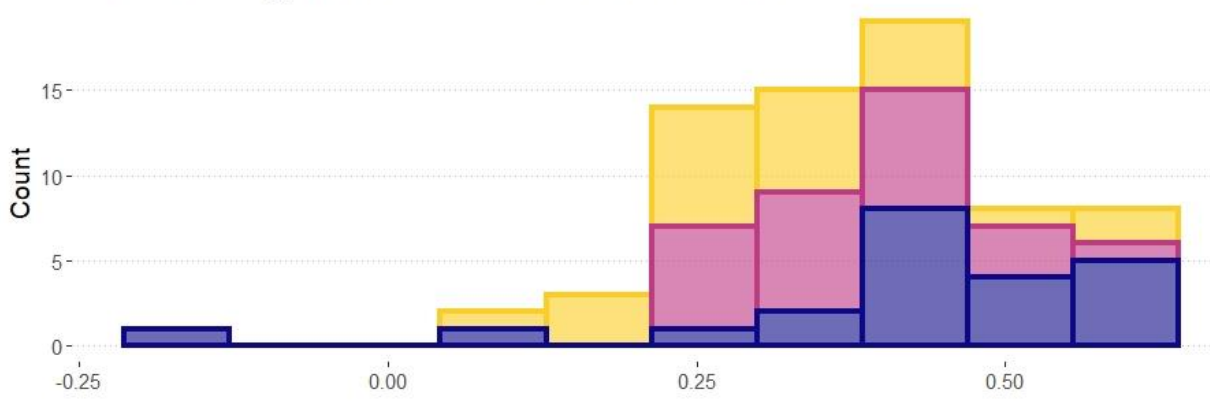

Distribution of Kappa for the Classification of the Likelihood Matrix Result

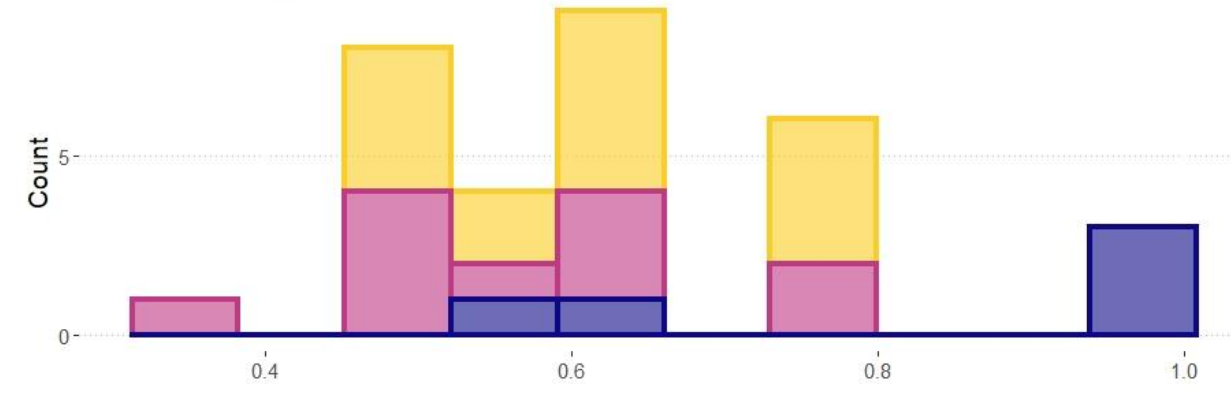

Distribution of Kappa for the Likelihood of Impact Classification

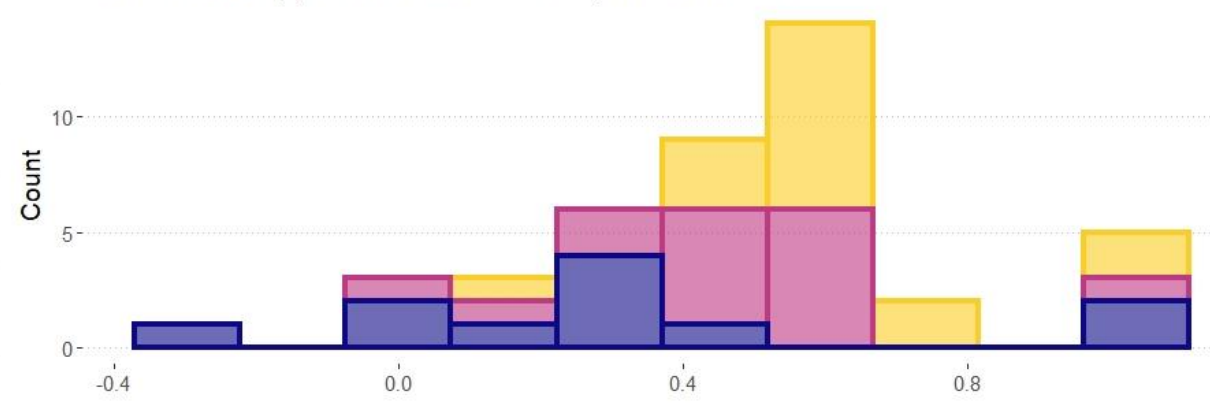

Distribution of Kappa Agreement Statistic for the Classification of Final Risk Rating

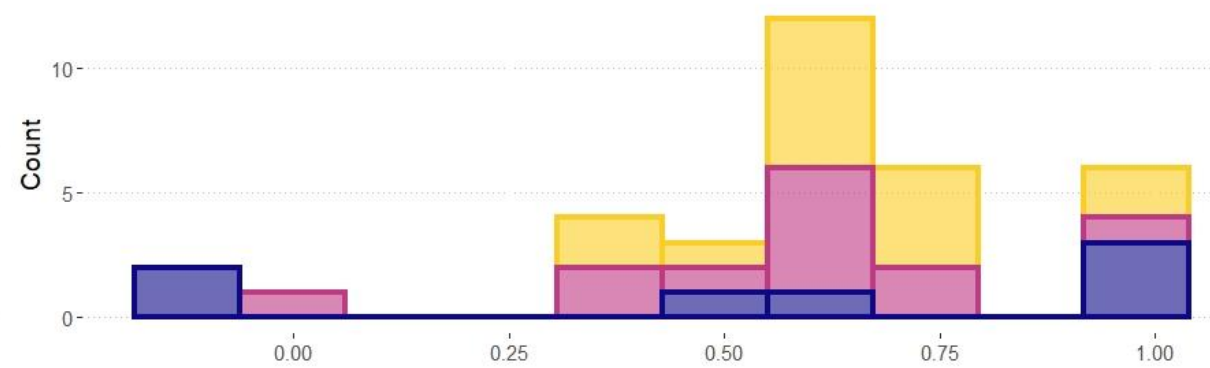

Matching Method $\square 2.3 \mathrm{~m} \square 4.5 \mathrm{~m} \square$ Adaptive

Figure 53: Distribution of Kappa Agreement Statistics for each ISA tree risk assessment classification 


\section{Threat Detection}

Trees were assigned a threat detection result (Y, yes/ N, no) based upon their threat detection result, if the critical height was greater than or equal to 0 , the tree was assigned "yes", otherwise trees were assigned "no". We analyzed results both within and across matching methods, and here $\mathrm{N}=48$.

Within the 2.3 m matching routine, four of 16 lidar derived models possessed Kappa values of 1 , demonstrating perfect agreement with the validation dataset and three of those four were found to be significant by the p-value of Kappa (each with a p-value $=0.0286$ ). In total, 13 of 16 models were found to be significant by the probability $>\mathrm{z}$ measure for Kappa. For a visualization of the distribution of Kappa values for the Threat Detection classification within the $2.3 \mathrm{~m}$ matching routine, see Figure 52.

Meanwhile, within the $4.5 \mathrm{~m}$ matching method, all 16 models were found to be significant with a maximum p-value of 0.0322 and, Kappa values of significant models ranged from 0.2649 to 0.8936 , see Figure 52. Likewise, within the adaptive matching method, all 16 models were found to be significant with a maximum p-value of 0.0424 , while Kappa values of significant models ranged from 0.3396 to 1.0, see Figure 52.

Across the matching methods, only two models' Kappa value for the agreement of Threat Detection were found to be not significant and both were single canopy models, TIN DEM normalized, decimated point cloud, and our tree base approximations, $\mathrm{CP}$ and $\mathrm{XY}$, as matching features. Additionally, we conducted an ANOVA on the Kappa values for Threat Detection classification and found a statistically significant difference in the means of Kappa values (p value $=0.009)$. The effect tests concluded that the canopy type modeling parameter had a significant effect on the mean value of Kappa $(\mathrm{p}$ value $=0.0007)$, where the single canopy model 
performed significantly better than the divided canopy model. Least square means showed that single canopy models averaged a Kappa value of 0.8222 , while divided canopy models averaged a kappa value of 0.6323 .

\section{Likelihood of Failure}

Arboricultural "post-storm study" literature has generally found tree height and DBH to be the most significant factors in tree failure, so a Likelihood of Failure measure was calculated as tree height multiplied by tree DBH (Kane 2008, Peterson 2007, Gardiner et al. 2008, Dahle et al. 2017). The distribution of matched trees' surrogate Likelihood of Failure measure is displayed in Figure 26. After creating this Likelihood of Failure measure variable, we recoded the resulting values into the categories, "Improbable", "Possible", "Probable", and "Imminent" from the International Society of Arboriculture's tree risk assessment BMPs (Smiley et al. 2017). See Figure 27 for the if/then conditional formula used to classify our likelihood of failure measures into the ISA's categories. First, we will look at the results of our contingency analyses within each matching routine, before looking across the matching routines. Also, here $\mathrm{N}=70$, since all models were able to predict DBH from tree height and crown area, and the Likelihood of Failure measure was the interaction of tree height and the predicted DBH. Yet, two models were not able to calculate Kappa based upon their contingency tables.

For trees matched by the models within $2.3 \mathrm{~m}$ matching method, the distribution of our surrogate Likelihood of Failure measure is available in the Figure 26. Nineteen of 24 models were found to be significant and two models were not able to compute Kappa based on the contingency analysis. The significant models' Kappa values ranged from 0.4 to 0.6052 , see Figures $52 \& 53$ for the distributions of Kappa values within the $2.3 \mathrm{~m}$ matching routine. 
For the trees matched by models within the $4.5 \mathrm{~m}$ matching method, the distribution of our surrogate Likelihood of Failure measure is available in the Figure 26. All 24 models were found to be significant with a maximum p-value of 0.0212 and, the Kappa values ranged from 0.2641 to 0.6254 , see Figures $52 \& 53$ for the distribution of Kappa values within the $4.5 \mathrm{~m}$ matching routine.

Lastly, for the trees matched by models within the adaptive matching method, the distribution of our surrogate Likelihood of Failure measure is available in the Figure 26. Seventeen of the 24 models were found to be significant, and Kappa values ranged from 0.1030 to 0.625 , see Figure $52 \& 53$ for the distribution of Kappa values within the adaptive matching method.

For trees matched across all matching routines, the distribution of our surrogate measure for the Likelihood of Failure is available in the Figure 26. The distribution of Kappa values of agreement for the Likelihood of Failure classification across matching methods is displayed in Figure $52 \& 53$. Note, two models have been dropped due to not being able to compute a Kappa value. Thus, $\mathrm{N}=70$ and eight models were deemed not significant, leaving 62 significant models.

The overall ANOVA of the Kappa values of agreement for the Likelihood of Failure classification discovered a statistically significant difference in the means of Kappa values ( $p$ value $=0.0022)$. The effect tests conclude that the point density $(\mathrm{p}$ value $=0.0073)$, DEM for point cloud normalization ( $\mathrm{p}$ value $=0.0018$ ), and the Match Method $(\mathrm{p}$ value $=0.0486)$ had significant effects on the mean value of Kappa. KNN models outperformed TIN models, with a Kappa value of 0.4236 compared to 0.3234 . Decimated models outperformed full density models, with an average Kappa of 0.4173 compared to 0.3350 . The 2.3 m matching routine 
performed best with an average Kappa of 0.4134 , while the adaptive method performed the worst with a Kappa of 0.3252 , and the most lenient matching routine, the $4.5 \mathrm{~m}$ method, obtained a Kappa value of 0.3895 .

\section{Likelihood of Impact}

We calculated the Likelihood of Impact as the length of electric line that fell inside a buffer around each tree's base that was equivalent to the height of the tree. We only calculated this measure for trees that were identified in the Threat Detection protocol. The calculation was completed in ArcGIS Pro using the "summarize within" tool and the distribution of our Likelihood of Impact measure is displayed as a distribution both for all matched trees and for matched trees that were deemed threats by our Threat Detection workflow in Figure 26. The measures were then recoded into the ISA's tree risk assessment BMP method's four categories, "Very Low", "Low", "Medium", and "High" (Smiley et al. 2017). See Figure 53 for the distribution of matched trees among the Likelihood of Impact classification categories.

A contingency table analysis was used to obtain the Kappa agreement statistic for the agreement of the Likelihood of Impact classification. The Likelihood of Impact measures equals 0 when a matched tree cannot come into contact with electrical conductors, as was the case for most of the matched trees. The treetops matching feature cannot be included in this analysis, as the Likelihood of Impact is dependent upon the tree base location and elevation. Additionally, five models could not compute Kappa based upon their contingency table and thus, $\mathrm{N}=43$.

The distribution of the Likelihood of Impact measure within 2.3 m matching routine is provided in Figure 26. The distribution of matched trees among the classification levels for the Likelihood of Impact classification are available in Figure 53. The Kappa values for the agreement of field and lidar derived for matched trees are displayed as a distribution within each 
matching method, in Figures 52. Within the $2.3 \mathrm{~m}$ matching method, Kappa values ranged from 0.3333 to 1.000 and, two models obtained Kappa values of 1.0, thus achieving "perfect" agreement. Although, neither were deemed significant ( $p$-value $=0.0786$ ). In fact, no models were found to be significant within the $2.3 \mathrm{~m}$ matching regiment.

Next, the distribution of the Likelihood of Impact measure within the $4.5 \mathrm{~m}$ matching routine is provided in Figure 26. The distribution of the 4.5 m matching method's matched trees among the classification levels for the Likelihood of Impact classification are available in Figure 53. The Kappa values for the agreement of field and lidar derived classifications within the 4.5 matching routine are displayed as a distribution in Figure 52. The Kappa values ranged from 0.000 to 1.000 . Eleven of the 16 models were significant, and one of these significant models obtained a Kappa value of 1.000 .

Likewise, the distribution of the Likelihood of Impact measure within the adaptive matching regiment is provided in Figure 26. The distribution of the adaptive matching method's matched trees among the classification levels of the Likelihood of Impact classification are available in Figure 53. The Kappa values are displayed as a distribution in Figure 52. The Kappa values ranged from 0.2105 to 1.000 and, all but one of the models were significant.

Finally, the distribution of the Likelihood of Impact across all matching methods is provided in Figure 26. The distribution of matched trees among the classification levels of the Likelihood of Impact classification across matching methods is available in Figure 53 and the Kappa values for the agreement of field and lidar classifications are displayed a as distributions in Figure 52. Twenty-seven of the 43 models, where Kappa could be calculated, were statistically significant. Although, no models within the $2.3 \mathrm{~m}$ matching method models were significant. 
The ANOVA of the Kappa values of agreement for the Likelihood of Impact classification from lidar derived trees compared to field trees revealed a statistically significant difference in the means of Kappa values ( $\mathrm{p}$ value $=<0.0001$ ). The effect tests concluded that the DEM for point cloud normalization $(\mathrm{p}$ value $=<0.0001)$, point cloud density $(\mathrm{p}$ value $=0.0140)$, matching method $(\mathrm{p}$ value $=0.0193)$, and the matching feature $(\mathrm{p}$ value $=0.0334)$ had statistically significant effects on the mean value of Kappa. TIN models outperformed KNN models averaging a Kappa value of 0.6833 , compared to 0.3368 . Decimated models outperformed Full density models averaging a Kappa value of 0.5673 , compared to the latter's 0.4160 . The adaptive matching method performed best, averaging a Kappa of 0.6139 , while the $2.3 \mathrm{~m}$ matching method averaged a Kappa of 0.3311 , and the $4.5 \mathrm{~m}$ matching method averaged 0.4751. The CP method (center point of polygon) of tree base location approximation outperformed the XY method (average x,y of all points in a given "treeID") averaging a Kappa value of 0.5554 , compared to the latter's 0.4146 .

\section{Likelihood Matrix}

The ISA tree risk assessment BMPs use two categorical measures, one of the Likelihood of Failure and the other of the Likelihood of Impact as inputs into a larger "Likelihood Matrix", where the result is the "Likelihood Measure" and the possible categories are "Unlikely, Somewhat Likely, Likely, and Very Likely" (Smiley et al. 2017). The conditional if/then formula used to mimic the operation of the ISA's Likelihood Matrix is available in Figure 34. A contingency table analysis was used to obtain the Kappa agreement statistic for the results of the Likelihood Matrix of the matched lidar derived and validation trees. Also, here initially $\mathrm{N}=48$ because models using the treetops matching feature were excluded in the Likelihood of Impact 
classification. Yet, since Kappa could not be calculated from 11 of the contingency tables, here $\mathrm{N}=37$.

The distribution of Kappa values for the agreement of the Likelihood Matrix classification of matched field and lidar derived trees for models utilizing the $2.3 \mathrm{~m}$ matching routine are displayed in Figure $52 \& 53$. All models were found to be significant, with a maximum p-value of 0.0043 . In this matching routine, nine of 16 models were capable of calculating Kappa based upon there contingency table analysis. Additionally, five of the nine models obtained Kappa values of 1.000, demonstrating "perfect" agreement.

The Kappa values for the Likelihood Matrix classification of matched field and lidar derived trees for the $4.5 \mathrm{~m}$ matching routine are displayed as a distribution in Figures $52 \& 53$. All models were found to be significant with the maximum p-value of 0.0002. The Kappa values ranged from 0.3743 to 0.7843 . Within this matching routine three models were dropped due to Kappa not being able to be calculated based upon the contingency table analysis.

Within the adaptive matching routine, The Kappa values for the agreement of matched field and lidar derived trees' Likelihood Matrix classification are displayed as a distribution in Figures $52 \& 53$. Again, all models were found to be significant with a maximum p-value equal to 0.0010 . Within this matching routine, the Kappa values ranged from 0.5275 to 0.7857 . Also, a model was dropped due to Kappa not being able to be calculated.

The distribution of Kappa values of agreement for the Likelihood Matrix, across all matching routines is displayed in Figures 53. Note, $\mathrm{N}=37$ because, a total of 11 models were not capable of calculating Kappa. Six were from the $2.3 \mathrm{~m}$ matching method, two from the $4.5 \mathrm{~m}$ matching method, and one from the adaptive matching method. All models where Kappa could 
be calculated were found to be statistically significant and the Kappa values across matching methods ranged from 0.3743 to 1.0 .

The overall ANOVA of the Kappa values of agreement for the classification results of the Likelihood Matrix demonstrated a statistically significant difference in the mean of Kappa values ( $\mathrm{p}$ value $=0.0002)$. Effect tests conclude that the matching method $(<0.0001)$, canopy modeling parameter (0.0031), and the DEM used for point cloud normalization (0.0084) had significant effects on the mean of Kappa values. The $2.3 \mathrm{~m}$ matching method performed the best with an average Kappa value of 0.8258 . The adaptive matching routine outperformed the $4.5 \mathrm{~m}$ method, averaging a Kappa value of 0.6670 , compared to the latter's 0.6120 . Models using a single canopy type outperformed the divided canopy models, averaging a Kappa value of 0.7326 , compared to 0.6612. TIN DEM normalized models outperformed KNN DEM normalized models, averaging a Kappa value of 0.7353 , compared to the latter's 0.6446 .

\section{Consequences of Impact}

Our "Lines" layer in ArcGIS Pro, representing the powerlines, were assigned customer number values, such that the Consequences of Failure could be modeled based upon the potential of each tree's failure and impact. See Figure 35 for a visualization of the "lines" feature. The recode formula in Figure 36 details how customer count was converted into ISA's tree risk assessment BMPs Consequences of Impact categories (Smiley et al. 2017).

The contingency analysis, available in Figure 54 consists of model predicted consequences compared to field data predicted consequences. The Kappa value of 1.000 demonstrates "perfect" agreement which was necessary in this step, as it would not be beneficial to have two different models match the same tree, capable of contacting the same line, with 
different consequences. Thus, this perfect agreement is necessary, and the grid model was a constant in this study.

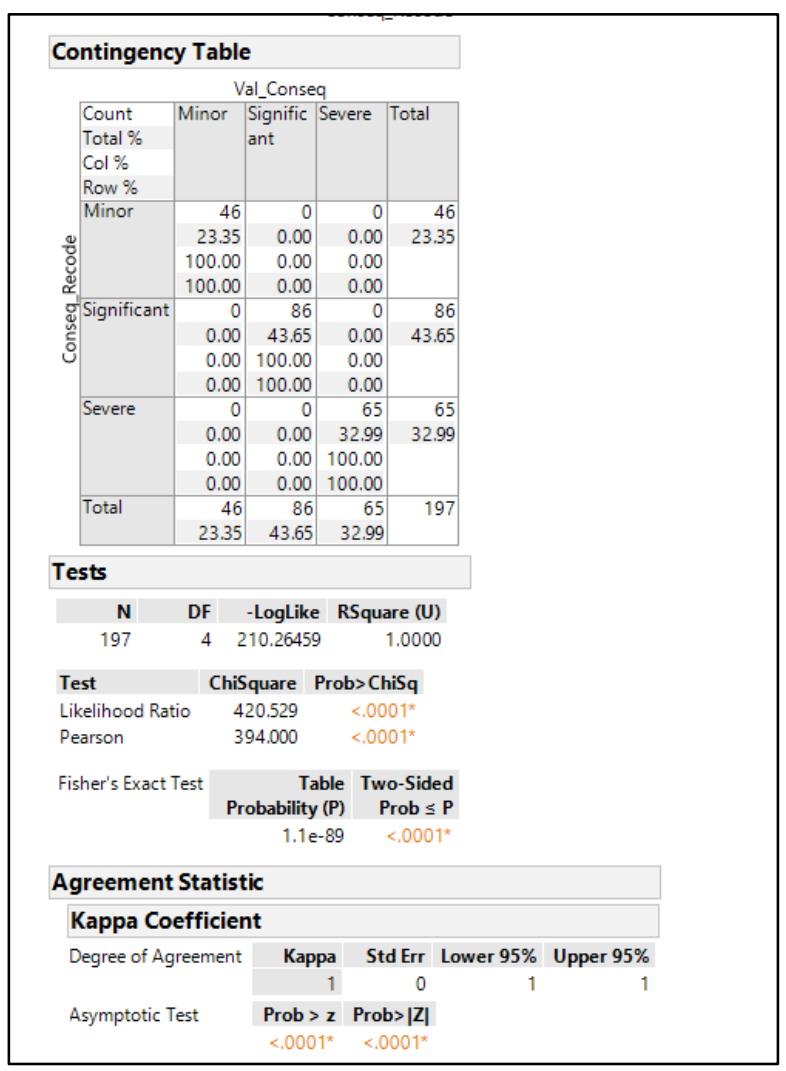

Figure 54: Contingency Table Analysis for Consequences of Impact

\section{Final Risk Rating}

The International Society of Arboriculture's tree risk assessment BMPs uses the output of the Likelihood Matrix along with the Consequences of Failure as inputs for Final Risk Rating matrix (Smiley et al. 2017). Here the Consequences of Failure are the predicted number of customers that would be without of power should tree failure and impact occur. The power line model with the number of customers at each location was held the constant for each model, such that there was no variability in Consequences of Failure between any two models. The result of the Likelihood Matrix was calculated as the combination of the Likelihood of Failure and 
Likelihood of Impact classifications. The Final Risk Rating combines the result of the Likelihood Matrix with the Consequences of Impact category to obtain the Final Risk Rating where, the possible outputs are, "Low", "Moderate", "High", and "Extreme". The conditional if/then formula used for the adaptation of the ISA's Final Risk Rating calculation is available in Figure 37. Contingency table analyses were used to obtain the Kappa agreement statistics for the agreement of Final Risk Rating classifications of field and lidar derived matched trees. Also, like the Likelihood Matrix classification before, initially $\mathrm{N}=48$ because models using the treetops matching feature were excluded yet, Kappa could not be calculated from 11 of the contingency tables, thus $\mathrm{N}=37$. Furthermore, the results from within each matching routine are examined before the results are examined across matching methods.

The Kappa agreement values for the Final Risk Rating classification within the $2.3 \mathrm{~m}$ matching method are displayed as a distribution in Figures $52 \& 53$. All models were statistically significant with a maximum p-value of 0.0130 , and of the nine models where Kappa could be calculated, five obtained Kappa values of 1.000 .

The Kappa agreement values for the Final Risk Rating classification within the $4.5 \mathrm{~m}$ matching method are displayed as a distribution in Figure 52 \& 53. Again, all models were found to be statistically significant, with a maximum p-value of 0.0007 . Two of the models possessed a Kappa of 1.0, while the minimum Kappa value was 0.4834 .

The Kappa agreement values for the Final Risk Rating classification within the adaptive matching method are displayed as a distribution in Figure $52 \& 53$. All models were found to be statistically significant, with a maximum p-value of 0.0097 . Three of the models possessed a Kappa of 1.0, while the minimum Kappa value was 0.5238. 
Lastly, the Kappa values across all matching methods are displayed as a distribution in Figure 53. Note, $\mathrm{N}=37$ since, in total 11 models were dropped, six from the $2.3 \mathrm{~m}$ matching method, two from the $4.5 \mathrm{~m}$ matching method, and one from the adaptive matching method. All models where Kappa could be calculated were found to be statistically significant and the overall ANOVA of the Kappa agreement values for the Final Risk Rating classification was statistically significant $(\mathrm{p}$ value $=0.0037)$. The effect tests concluded that the DEM used for point cloud normalization $(\mathrm{p}$ value $=<0.0001$ ) had a significant effect on the values of Kappa for Final Risk Rating classification where, TIN normalized methods outperformed the KNN normalized methods, averaging a Kappa value of 0.8562 , compared to the latter's Kappa value of 0.6477.

\subsection{Discussion}

This section will first discuss the results of our statistical analysis, working through the same framework as our results section. After we have concluded with our discussion of the statistical analyses, we will begin a discussion of the project in a broader sense, Attempting to situate this study within the literature, as well as state any improvements that could be made to similar future projects.

\section{DEM \& Pole Location}

$D E M$

For the DEM accuracy assessment, we solely utilized data obtained from the first attempted field data acquisition using the Spectra SP80. While we did not include the data pertaining to tree base locations, we retained the location and elevation information of points along road and of the electric power poles. After determining that our individual tree detection 
and segmentation had drastically under-segmented the tree data, we abandoned this first attempt at field data acquisition with only 15 points for DEM validation. Nine were located at the base of the power poles and six along the road within the study area.

Ideally, we would have returned and collected location and elevation data for all of the electric poles, and more points along the road and in open areas in the middle of the ROW. However, the Spectra was not available once we had re-completed the individual tree detection and segmentation routines and we had to make do with the GPS data we already possessed. Thus, we utilized these 15 locations and their elevations for DEM accuracy assessment, since the poles locations were easily discernable, and the road locations provided a stable, flat area with no overhead tree canopy which allowed for the acquisition of high accuracy elevation data. When we compared these 15 Spectra GPS obtained elevations with the values at these locations in each of our three lidar-derived DEMs and the state's highest resolution DEM, we found that the state DEM (WV1to3m) was substantially higher in elevation $(\sim 30 \mathrm{~m})$ than the other models and the Spectra measurements. The cause of this difference has yet to be determined, but it is suspected that the error is in the state's DEM and not our DEMs since our DEMs aligned much more closely with the measures from the Spectra's elevation measurements.

the DEM interpolated via the kriging method demonstrated many visual artifacts when displayed as a raster and was thus dropped from further analysis. this is not to suggest that the kriging interpolation method is not suitable for DEM generation from an UAS-lidar generated point cloud. Instead, this issue was likely user error on the part of the researcher, and more time 
would have been necessary to understand and tune the parameters for the kriging interpolation method.

\section{Pole Locations}

Li et al.'s (2012) individual tree detection and segmentation algorithm was used to automatically detect and segment electrical pole structures. The workflow for this process mirrors the process of individual tree detection and segmentation, see Figure 21. After the classification of vegetation, building, and ground classes, the point cloud was filtered to the classes "never classified", "unclassified", and "ground" classes. Then, after little tuning, Li et al's (2012) method quickly identified all the poles in the AOI and provided each with a unique identifier. This use of Li et al.’s (2012) algorithm for the purpose of identifying electrical infrastructure, again, appears to be the first in the literature. Possibly, Li et al.'s (2012) algorithm may be able to find other vertical structures from within lidar point clouds, or it may be able to be modified in some fashion for that task. In particular, structures such as cellular and radio towers, and large transmission electric pylons, where even tallest vegetation could be ignored via the algorithm's minimum height parameter, seem to be a natural extension of the algorithm's applications.

For the assessment of electric power pole locations, we compared these lidar derived pole locations to the two sources of GPS data available from our first, failed field data collection and the subsequent successful field data collections, using the Spectra SP80 survey grade GPS, and the Trimble GeoXT handheld GPS, respectively. This comparison was complicated by the fact that we had different subsets of the poles for each of the GPS location measurements. However, the lidar pole dataset included all the poles in the area of interest. The Spectra and Trimble datasets shared four poles, the Spectra and the lidar datasets shared eight poles, and the Trimble 
and the lidar datasets shared five poles and thus, comparisons between methods utilized a different number of poles and different poles.

Preferably, we would have data for all the poles with both the Spectra and the lidar, allowing for the comparison of just those two sources of pole locations. Yet, that was not the case and, we included the Trimble GeoXT pole locations in the analysis to verify that the location data obtained with it was more accurate than that of the lidar, due to its future use in the validation of tree base locations. As seen in the results, Welch's ANOVA was used to control for different variances and no significant differences in pole locations were found across the different sources of pole location data.

Furthermore, the Spectra and Trimble had the smallest mean difference of $1.7147 \mathrm{~m}$ in pole locations, while the lidar differed from the Spectra by $2.1606 \mathrm{~m}$ and the Trimble by 3.1946 m. This suggested that either of the GPS methods were more accurate than the lidar used in this research. Additionally, the pole locations served as a measure of the $\mathrm{x}, \mathrm{y}$ or spatial accuracy of the point cloud, since they were rather obvious features which allowed for the acquisition of high accuracy location data. We used the mean difference between our most accurate method, the Spectra, and the lidar to determine the amount of position error inherent in the point cloud. We concluded that there was $2.1606 \mathrm{~m}$ of position error inherent in our point cloud.

\section{Individual Tree Crown Detection and Segmentation Metrics}

Our overall mean Tree Detection rate of 0.8578 is comparable to the literature, see Figure 46 (Wallace et al. 2014, Vauhkonen et al. 2012, Kaartinen et al. 2012, Esyn et al. 2015, Pirotti 2010). Furthermore, within our models the mean of single canopy models was 0.5334 , While the 
mean for divided canopy models was 1.18 . Again, this is generally consistent with the literature. Likewise, our results also concur with Wallace et al. 2014, where the difference in Detection rate between models decimated to $50 \mathrm{ppm}^{2}$ and full density models was for practical purposes nonexistent (Wallace et al. 2014).

The overall mean for Tree Matching rate in our study was 0.1144 which, when compared to literature is low, as most studies appear to achieve between $30-60 \%$ matching (Pirotti 2010, Esyn et al. 2015, Vaukonen et al. 2012, Kaartinen et al. 2012). Furthermore, our matching routines' distance used clearly had an effect on matching rates: models using the $2.3 \mathrm{~m}$ matching distance from Pirotti (2010) averaged 0.0576; models using the $4.5 \mathrm{~m}$ matching distance averaged 0.1770; and models using Esyn et al.'s (2015) height adaptive matching distance averaged 0.1085 . none of the methods were capable of performing at levels comparable to literature. Likewise, our other tree matching metrics were similar to the Tree Matching rate, with low overall means for Precision (0.1411) and Recall (0.1144) when compared to literature, see Figure 47. We calculated a Kappa agreement statistic for the matching of lidar derived trees to field trees in accordance to the methods provided in the literature, see Figure 49 (Pirotti 2010, Pirotti et al. 2017). We found our overall mean for the Kappa of lidar derived trees and field trees to be -0.6749 , which was comparable to Pirotti 2010, but was substantially lower than Kappa values presented in Pirotti et al. 2017.

We suspect that independent georeferencing errors in the point clouds of each separate flight line could be the cause of the matching issues. We attempted to shift the merged point cloud to obtain better matching rates but in achieving additional matches in one location, the shift would lead to the loss of matches in a different location. Thus, the solution appears to be to 
thoroughly examine and mend any georeferencing errors in each flight line prior to merging, classification, or spatial analysis.

The overall mean for lidar derived tree location RMSE was 2.3874 m, see Figure 49. Although this value was constrained by the matching distance, where the models using $2.3 \mathrm{~m}$ distance from Pirotti (2010) had a mean RMSE 1.5494 m; those using the $4.5 \mathrm{~m}$ matching distance had a mean RMSE of 3.0301 m; and, those using Eysn et al.'s (2015) height adaptive matching distance averaged an RMSE of $2.5829 \mathrm{~m}$. These tree location RMSE appear comparable to literature (Kaartinen et al. 2012, Eysn et al. 2015, Vauhkonen et al. 2012, and Wallace et al. 2014). Although, of these studies, Wallace et al. (2014) is the only other study to utilize UAS-based lidar.

This study also computed the Omission and Commission error rates for individual tree detection and segmentation according to Pirotti et al. (2017) and are summarized in Table 5. Commission Error rate is also available in Figure 48. We found an overall mean for Omission rate of 0.5258 and, 0.4085 for the Commission rate. Furthermore, within single canopy models, Omission and Commission rates were, 0.6319 and 0.3087 , respectively. While divided canopy models had Omission and Commission error rates of 0.4197 and 0.5083 , respectively. These values were, again, generally comparable to the literature (Esyn et al. 2015, Vauhkonen et al 2012, Kaartinen et al. 2012). Furthermore, Eysn et al. (2015) proposed an evaluation index of matching rate over commission rate, also available in Figure 48. We calculated this evaluation index for each of our models, and the overall mean was 0.2863 , and the means within each matching routine were $0.1333(2.3 \mathrm{~m}), 0.4564(4.5 \mathrm{~m}), 0.2691$ (adaptive). Unfortunately, Esyn et al. (2015) did not provide the results of this index for their own work. So a direct comparison of our values and theirs is not possible. 
Lastly, we decided to visualize two distinct sets of our field trees. The first, a subset of our field trees which were determined to be vegetation threats by the field tree based Threat Detection workflow, but were not matched by any of our lidar models. We called this subset "Undetected Threats" and, this subset was composed of two slightly different datasets, due to differences in the field tree's KNN DEM based and TIN DEM based Threat Detection workflows. However, the two share a large portion of their data, as all five of the undetected threats in the TIN DEM dataset are featured in the KNN DEM dataset (5 of 7). Descriptive statistics for tree measures, tree risk measures, and distribution among the ISA's tree risk assessment classifications are available in Figures 55, 56, and 57.

The second subset of field trees are those that were matched by at least one of our lidar models $(\mathrm{N}=51)$. For this dataset we provided descriptive height and diameter data in Figure 58 . since these were identified by potentially many models, their elevation measures, risk measures, and ISA classifications vary by model, and thus those measures were not summarized here. this dataset was visualized by field plot. 


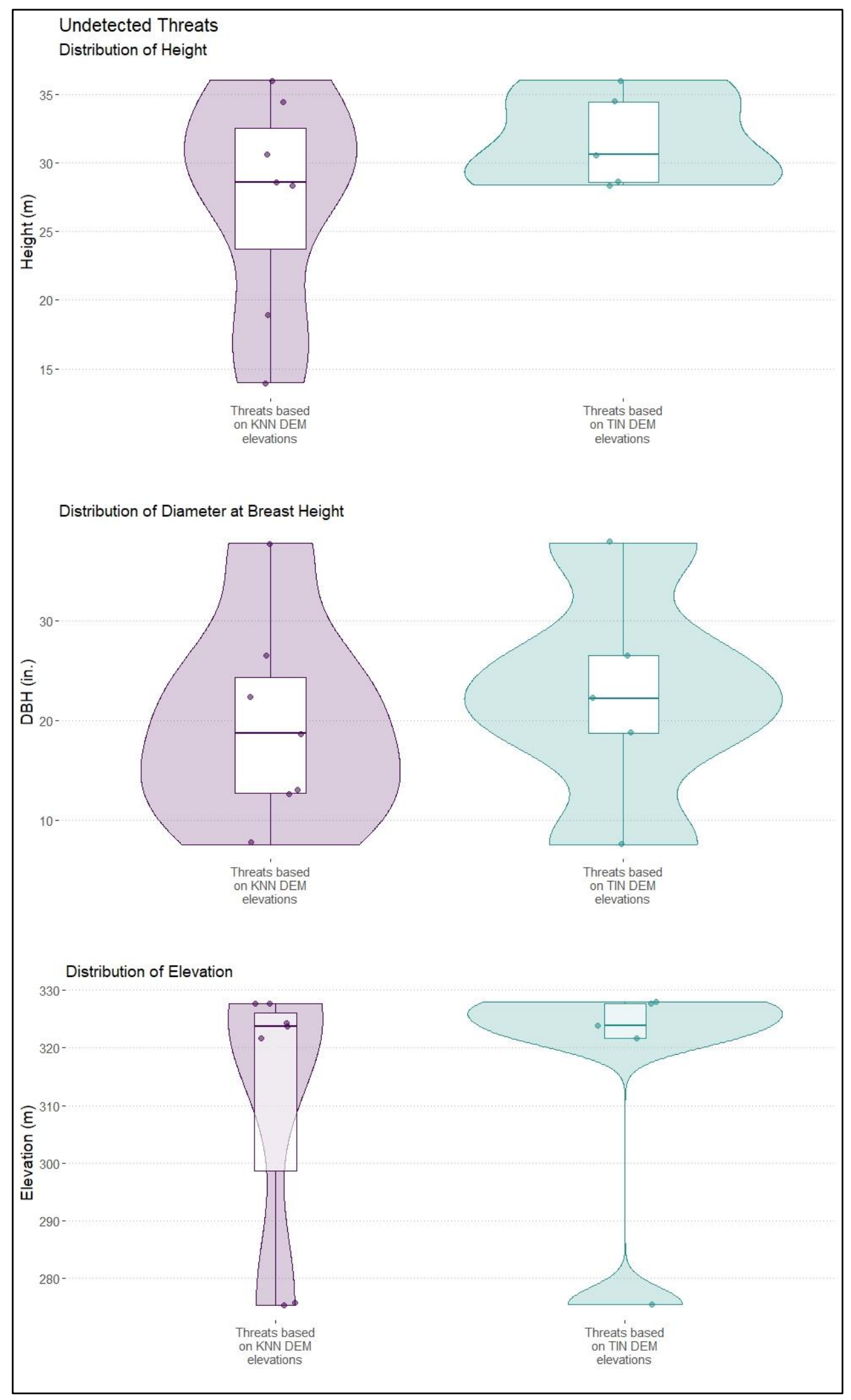

Figure 55: Undetected Threats: Field Measures 


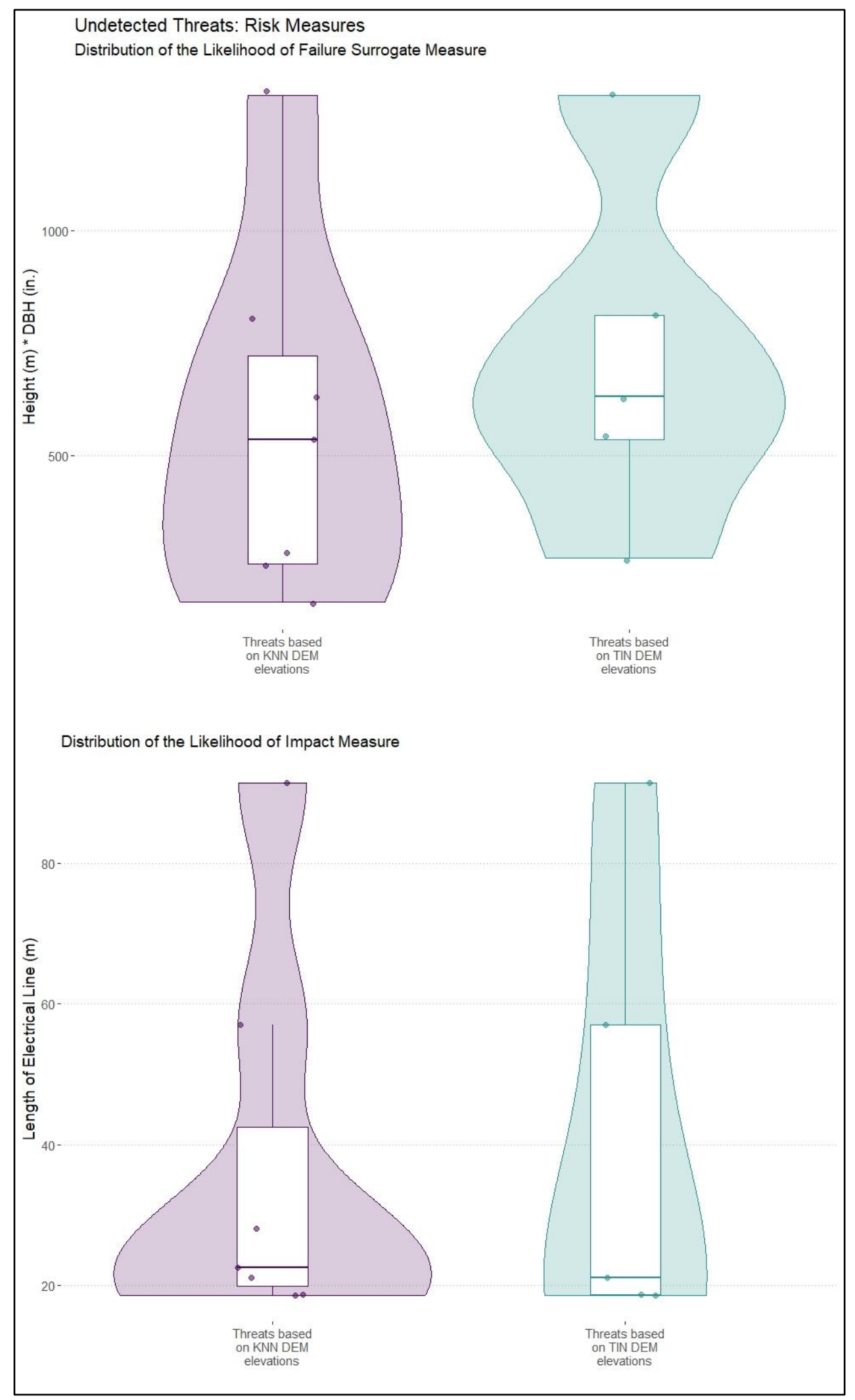

Figure 56: Undetected Threats: Risk Measures 


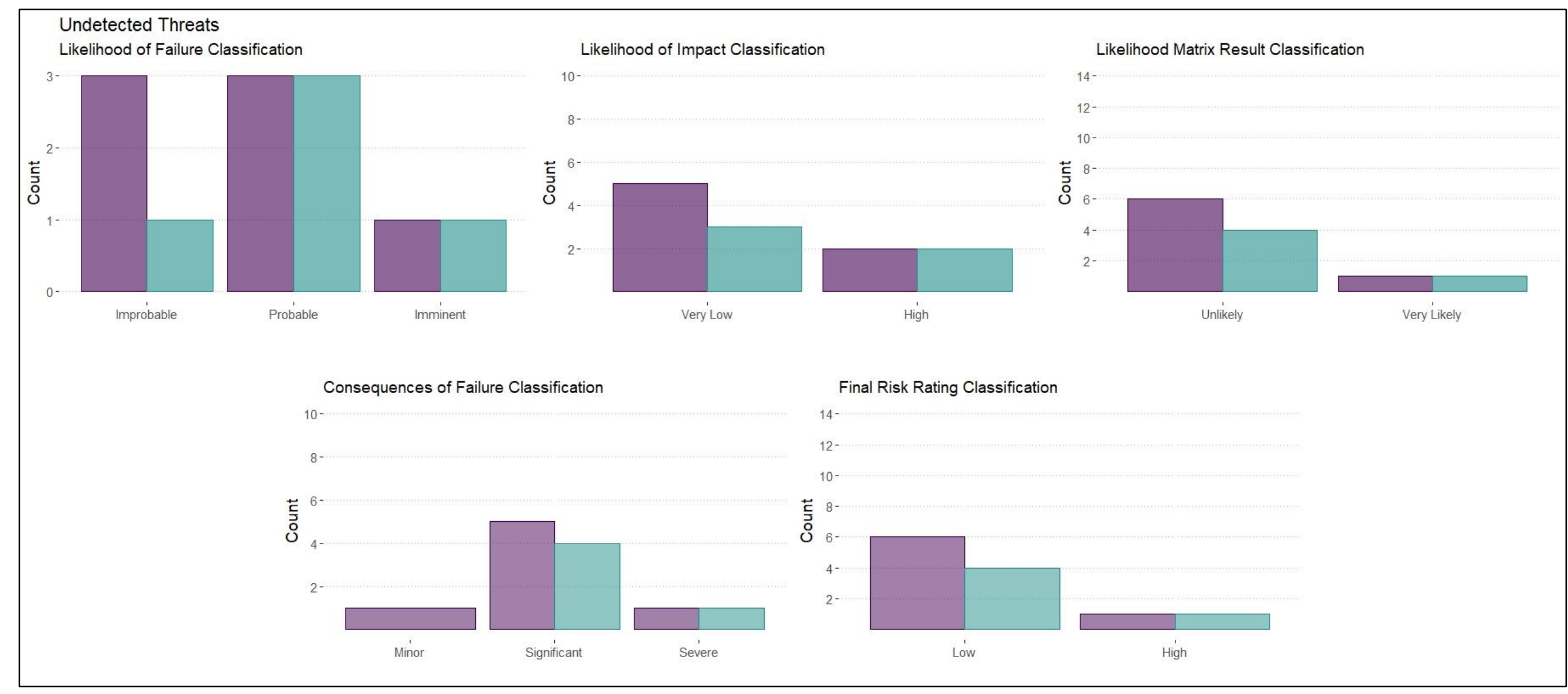

Figure 57: Undetected Threats: Distributions for the ISA's Tree Risk Assessment Classifications 




Distributions of Diameter at Breast Height



Figure 58: Unique, Matched Field Trees 


\section{Division of the point cloud by vegetation canopy type}

Larsen et al. (2011) suspected better performance could be expected in individual tree detection and segmentation routines by first dividing an image into its different stands, then applying different parameters for each stand type. We therefore hypothesized that individual tree detection and segmentation from within a lidar point cloud would benefit from the same division into stand types. Within the utility forest, open-grown "yard" trees and areas of lawn present a different environment than that of any closed canopy forested area. Thus, the point clouds were divided based on a quick manual interpretation of these two contrasting canopy types.

The individual tree detection and segmentation routines within the $2.3 \mathrm{~m}$ matching method were subject to ANOVAs for tree base location, tree base elevation, tree height and predicted tree DBH. Effect tests and least square means were used to determine that divided canopy models performed better than single canopy models in both tree height assessment and demonstrated a trend towards better for DBH prediction. Despite this, and counterintuitively, single canopy models performed better in Threat Detection classification and the Likelihood Matrix classification agreement. It was our expectation that because the divided canopy models were more accurately representing the height and $\mathrm{DBH}$ of matched trees, that their classification results would also more likely to agree with those of our field tree's results. The increased performance of single canopy models in Threat Detection and the Likelihood Matrix classifications could be, at least partially, due to the fact that these models under-segmented trees, thereby only capturing some combination of the larger, more obvious, or more isolated trees. These trees too would likely be easier to classify correctly trees. In the future, the division of the point cloud would ideally be automated, potentially by utilizing some sort of tree or treetop per area measure. 


\section{Tree Base Location}

The analysis of tree base locations was complicated by two matters. The first, to assess the difference between our predicted lidar derived tree bases and the field acquired GPS tree bases, we had to utilize matching routines which possessed distance based matching requirements. Thus, as the matching distance becomes smaller, it restricts the amount of possible position error in the matched trees at the expense of the matching rate. See Figure 49 to see tree base location error by match method. Given this relationship, we examined the results of tree base location approximation methods within each matching routine, as well as across the matching routines. the ANOVA found no significant difference in the distance between matched tree bases using either of the two base approximation methods.

While the overall ANOVAs within both the $4.5 \mathrm{~m}$ and adaptive matching methods were not significant (p-values: 0.8585 and 0.5601 , respectively), within the strictest of matching methods, the 2.3 m routine from Pirotti (2010), the ANOVA found a significant difference in the mean distance of matched tree base locations ( $p$-value $=0.0465)$, and the effect tests determined the difference was due to the density parameter ( $\mathrm{p}$-value $=0.0131)$. The full density models possessed a mean difference of $1.3577 \mathrm{~m}$ in matched tree locations, and the decimated models possessed a mean difference of $1.5847 \mathrm{~m}$. This suggests that the full density models are $\sim 0.2 \mathrm{~m}$ more accurate at determining tree base location, when using Pirotti’s (2010) matching routine. Thus, our experience is similar to Wallace et al.'s (2014), who found full density models are only marginally more accurate than point clouds with $50 \mathrm{ppm}^{2}$, or not more accurate at all, and require more processing time to complete the detection and segmentation process (Wallace et al. 2014).

Furthermore, the most generous matching regiment, the $4.5 \mathrm{~m}$ method, generated slightly better Kappa agreement values for individual tree detection and segmentation, see Figure 49. 
However, this was at the cost of a trade off with positional accuracy, hence the higher RMSE of tree location for our $4.5 \mathrm{~m}$ matching method. Perhaps the effect of decimation on positional accuracy is captured in the $2.3 \mathrm{~m}$ matching method due to the restricted amount of position error allowed. On the other hand, the $4.5 \mathrm{~m}$ and adaptive matching routines allowance of greater matching distances, and thus the potentially larger errors in matched tree base locations were enough to obscure the effect of decimation, since even within the $2.3 \mathrm{~m}$ matching routine full density models were only on average $0.2 \mathrm{~m}$ more accurate.

The second complication in the assessment of tree location was that our lidar -based matching features included two point features generated as tree base approximation methods, and one point feature representing the highest point of each detected tree. Additionally, the treetops matching feature generally performed best within each of the matching routines. Yet, this matching feature was not compatible with the design of our Threat Detection workflow as it did not represent the approximated tree base, unlike the other two features. Based on this experience, in the future it may be best to perform the tree matching routines using only the lidar -derived treetops, while still attempting tree base approximations for later risk analysis, or foregoing the base approximation methods altogether and instead compensating for the use of the treetops through the addition of an error term within the calculation of the Critical Height. The latter possibility will be discussed further in the "Threat Detection" section of this discussion.

\section{Tree Base Elevation}

As with the DEM and pole location measurements, when we collected the field data for this project, we had intended to use the Spectra SP80 to obtain the tree base elevations for all trees in our field plots. When this was not feasible, we utilized an available Trimble GeoXT 
handheld GPS. However, this device could not provide us with elevation data for the points we collected.

Thus, we decided to compare our lidar -derived tree base elevations to the corresponding DEM elevation values at the locations of our field acquired tree base GPS points. We were able to complete this using ArcGIS PRO's "extract values to points" to extract both the TIN and KNN DEM values to the field data's tree base location point. TIN normalized lidar-derived models were compared to the TIN elevations at our field trees' base locations and KNN normalized lidar models were compared to the KNN elevations at our field trees' base locations. For future work it would be beneficial to utilize a more precise GPS with the capability of obtaining elevation information, to compare the lidar derived trees' base elevations and field measured tree base elevations. Even though our method for validation of tree base elevations is flawed, we conducted an ANOVA within each matching routine and another across the matching routines.

None of the ANOVAs were significant, yet the effect tests within each the $2.3 \mathrm{~m}$ and the adaptive matching routines identified that the DEM used for point cloud normalization had a statistically significant effect on the differences of elevation at matched tree bases ( $p$-values: 0.0384 and 0.0099 , respectively). Within the $2.3 \mathrm{~m}$ matching method, KNN normalized matched tree base elevations possessed a mean difference of $0.2765 \mathrm{~m}$, and TIN normalized matched tree bases possessed a mean difference of $0.0453 \mathrm{~m}$. Within the adaptive matching method, the mean difference of KNN normalized matched tree base elevations were $0.1773 \mathrm{~m}$ and, the mean difference of TIN normalized matched tree base elevations was $-0.0971 \mathrm{~m}$. Thus, both methods suggest that the TIN DEM normalized models were superior at predicting match tree base elevations. Interestingly, since difference was calculated as field data minus lidar -derived measurements, it appears that KNN normalized models consistently underestimate tree base 
elevation across matching methods. In contrast, TIN normalized models did not have a set direction error. Yet, these results should be viewed with caution, since the lidar -derived tree base elevations and the validation tree base elevations were extracted from the same DEMs. Thus, full validation of the assessment of tree base elevation from within lidar point clouds remains one of the more pressing issues to determine if our Threat Detection workflow can accurately detect the NERC Category $2 \& 3$ vegetation risks along distribution electric lines.

\section{Tree Height}

Lidar-derived tree heights were produced after point cloud normalization and individual tree detection and segmentation, so that the resulting points shapefile possesses the value for the highest point of that detected and segmented tree in its " $Z$ " field. These values were compared to the field data's tree height as assessed with a Nikon Forestry Pro handheld laser. Then after the matching routines, the matched trees' lidar derived tree heights were fitted against their field tree heights and their measures of R-square, correlation, and RMSE were provided earlier in Figure 50.

Again, all the correlation values across matching methods were significant (p-values: $<0.0001-0.0081)$ and the correlation ranged from a low of 0.8535 in an adaptive matching method model to 0.9934 in a 2.3 m matching method model. Interestingly, the RMSE of tree height ranged from $0.7970 \mathrm{~m}$ to $2.512 \mathrm{~m}$, each of these models came from within the $2.3 \mathrm{~m}$ matching routine. Yet, this routine possessed the lowest average RMSE (1.6554 m).

The differences of field tree height and lidar derived tree height were subjected to ANOVA, both within each matching method and across the matching methods. The ANOVAs of the $4.5 \mathrm{~m}$ and adaptive methods were both insignificant (p-values: 0.4996 and 0.3749 , respectively). Yet, the overall ANOVA of the difference of field tree heights and lidar derived 
tree heights from trees matched using Pirotti's (2010) 2.3 m matching routine was significant (pvalue $=0.0328$ ). Furthermore, the effect tests determined that the canopy modeling parameter had a statistically significant impact on the differences of field tree height and lidar derived tree height ( $\mathrm{p}$-value $=0.0015$ ). The divided canopy models were more accurate, with a mean difference in tree height of $-0.1301 \mathrm{~m}$, while the single canopy models possessed a mean difference in tree height of $-0.9292 \mathrm{~m}$. Since all values are negative (field - lidar), the data suggests that the lidar models overestimate tree height, which is not consistent with the majority of the literature (Brandtberg et al. 2003, Perrson et al. 2004, Heurich 2008, and Hyyppa et al. 2004). our experience appears to be more similar to that of Shrestha \& Wynne (2012) where the height of some portion of detected and segmented trees have been overestimated. this could be an example of the lidar simply being more accurate than the tool being used for its validation, since the Nikon Forestry Pro accuracy is $+/-15 \%$ of total tree height. Furthermore, the previous

literature had cited point density as the primary cause of the underestimation of height. Since the ANOVAs on the differences of field- and lidar-derived tree heights did not detect a significant difference due to the effect of point cloud decimation, our data seems to suggest that $50 \mathrm{ppm}^{2}$ is a sufficient point density for the accurate description of tree heights.

\section{Tree $D B H$}

Trees detected and segmented from lidar do not directly provide DBH. Originally, this study intended to use Hagan and Smith's (1986) method for tree diameter at breast height (DBH) prediction from crown area. However, when we utilized the coefficients provided in their study the results provided negative values for the predicted DBH. their coefficients were developed for loblolly pine (Pinus taeda) and not intended for use on other species or sites, and this may 
explain our low accuracy. Therefore, we developed a new set of models for predicting DBH from UAS-based lidar-derived tree metrics, from a subset of our data.

The subset of data that was used for the generation of new DBH prediction models, was the point cloud where: the AOI was divided based upon vegetation canopy type, the TIN DEM was used for point cloud normalization, the point density was decimated to $50 \mathrm{ppm}^{2}$, the matching feature were the treetops or "tops", and the matching method was "adaptive". This method matched 28 trees in the validation area, and these 28 trees were used to develop the DBH prediction formula. The $\mathrm{R}^{2}$, Adjusted $\mathrm{R}^{2}$, and RMSE were provided for the fit of each of the models to field measured DBH, in Table 3.

The lidar predicted DBHs for matched trees were fitted against their field measured DBHs to obtain measures of correlation and RMSE and these measures are provided Figure 51. Interestingly, the $2.3 \mathrm{~m}$ matching routine again had both the lowest and highest values, in this instance, for the correlation of matched trees' DBHs.

Then, the difference of field tree DBH and the lidar predicted DBH was calculated and subjected to ANOVA. The ANOVAs of DBH differences of validation and lidar derived trees were all insignificant and effect tests detected no statistically significant differences. Yet, a trend towards a difference due to the effect of the canopy modeling parameter was detected ( $\mathrm{p}$ value $=$ 0.0516) within the $2.3 \mathrm{~m}$ matching routine. Here, the average difference in DBH (field - lidar predicted) for the single canopy model was 2.1148 inches, while the divided canopy model had a difference of only 0.9366 inches. The trend suggests that the two-canopy model may be better at predicting the $\mathrm{DBH}$ of matched trees, within the 2.3 m matching method.

Furthermore, it appears that our model selection for DBH prediction may have slightly biased DBH prediction towards the adaptive matching routine, since the dataset used for the 
generation of the DBH prediction formula was from the adaptive matching method. This routine possessed the smallest RMSE of predicted tree DBHs, see Table 4. Additionally, this method had the highest mean and median of the correlation of predicted and field DBHs. The adaptive matching method also outperformed the other matching methods in RMSE of predicted diameter. Again, this is likely due to the fact that the predictive model was developed using an adaptive model's matched trees.

The application of the DBH prediction methods presented here to future lidar point clouds has the potential for further research. Also, given that the prediction method's parameters require tree height and crown area, it is possible that the DBH prediction formula could be applied to other remote sensing data types which provide these measures, such as Structurefrom-Motion point clouds.

\section{Risk Analysis}

We applied NERC category 2 and 3 "fall-in" categories in the attempt to identify trees along distribution line which are potential "fall-in" vegetation risks from a high-density UASlidar point cloud (NERC FAC-003). These are trees which, should they fall, possess the height to contact the electric conductor. Category 2 and 3 trees can be differentiated based upon their base location. Category 2 trees are "on-ROW" trees, typically their base is within the defined ROW edges and/or are within 15 feet from the center conductor for distribution UVM. Whereas, Category 3 trees are "off-ROW" where their base is located outside the defined ROW edge or further than 15 feet from the center conductor.

We identified the potential NERC Category $2 \& 3$ vegetation threats after the lidar derived trees were detected and segmented. We used our Threat Detection workflow to 
determine which trees could come into contact with electrical conductors, while considering the distance between, as well as height and elevation differences of the two objects (tree and power line). The same Risk Assessment and classification process as was conducted with the heights and locations from our field trees. The tree base elevations for our field trees were extracted from the same DEMs generated for the lidar-based approach. These elevation values were used as validation to compare the lidar derived measures against, since the GeoXT handheld GPS could not acquire elevation data. Thus, the accuracy of tree base elevation assessment from within a UAS-lidar generated point cloud needs to be fully validated against actual, field measured tree base elevations, before the Threat Detection and risk assessment classifications are applied in practice. A quantitative description of the field trees detected as NERC category $2 \& 3$ vegetation threats are available in Figures 41, 42, and 43.

We then utilized our Threat Detection results along with tree height and DBH in our attempt to adapt the ISA's tree risk assessment BMPs double matrix approach to tree risk assessment to our lidar derived tree information (Smiley et al. 2017). Given the quantitative nature of our lidar derived tree properties, this required the classification of our quantitative measures into the categories presented in the ISA's tree risk assessment BMPs.

Thus, our statistical analyses consisted of results from a series of contingency analyses for categorical data classification for each of our tree risk assessment classifications: Threat Detection, Likelihood of Failure, Likelihood of Impact, Consequences of Impact, and Final Risk Rating. We described the agreement between our lidar and field data as Kappa agreement statistics and, the Kappa values were displayed as distributions by matching method in Figure 48. 


\section{Threat Detection}

Our Threat Detection model represents a novel approach for the identification of NERC Category 2 \& 3 vegetation threats along distribution electric ROWs. There have been examples of "Encroachment Analysis" or "Proximity Analysis", where the distance between the conductors or the ROW and the vegetations is calculated, and if the vegetation is within that distance, it is categorized as a "threat" (Ussyhkin et al. 2011, Frank et al. 2010). Additionally, others have attempt to identify trees based on height and distance from the electrical infrastructure (Ko et al. 2012,). Yet, the ability of a tree to come into contact with an electrical conductor, should the tree fall, is at minimum based upon the tree's base location, height, and the elevation of the tree's base, as well as, the location of the electrical conductor and its height above the ground. we could not find a published model that has taken all of these factors into account.

The overall ANOVA on the Kappa values of agreement for Threat Detection classification was significant, and the effect tests concluded that the canopy modeling parameter had a significant effect on the mean value of Kappa ( $\mathrm{p}$-value $=0.0007)$, where the single canopy model performed significantly better than the divided canopy model, counter to what we had anticipated. The average Kappa value across matching methods, 0.72 , shows moderate to strong agreement. Yet, single canopy models averaged a Kappa value of 0.8222, while two canopy models averaged a kappa value of 0.6323 .

The single canopy models were observed to generally under-segment trees, evident from low detection rates, see Table 5. This would lead to only the more clearly defined, dominant trees being detected, and thus having the opportunity to be matched. Thus, single canopy models could essentially be matching the "easy" trees, where they are either tall or isolated or a combination of the two. Another possibility is that within the Threat Detection classification of 
trees, the 2.3 m matching routine models excelled matching trees in the "No" category. These trees are not capable of striking the line based upon the calculation of the Critical Height measure. Thus, correctly matching these trees would result in higher Kappa values for agreement but would provide little assistance in the identification of NERC Category $2 \& 3$ vegetation threats. If so, models utilizing the divided canopy type possessed higher matching rates, but misclassified Threat Detection more often, as is evident from the significant difference in means Kappa agreement values across methods. See Figure 46 for matching rates in this study.

Nevertheless, our Threat Detection workflow's full validation was hindered due to the aforementioned issue of our field trees' lack of field measured elevation data. Also, the additional obstacle of our UAS-lidar point cloud unsuccessful capture of conductor wires was overcome by using the height value of $9 \mathrm{~m}$ for all line features used to connect our lidar detected and segmented electric poles. We chose this $9 \mathrm{~m}$ height because most distribution electric lines are about 30 feet above the ground, or roughly $9 \mathrm{~m}$. Thus, the lines do not represent actual electric lines as they lack line sag and instead mimic the contour of the DEM but are $9 \mathrm{~m}$ higher. Although these issues could be rectified in subsequent research if the UAS-lidar system proves capable of capturing the electrical conductors.

an error term could be added to the calculation of the Critical Height in the Threat Detection process. This error term could incorporate error from numerous sources such as, spatial error inherent in the point cloud, error in DEM elevations, tree base approximation error, tree base elevations error, or tree height error either in combination or solo. This could potentially be used to develop an acceptable Threat Detection adjustment from the error term, where the range of the risk adjustment is equivalent to the sum of the errors from error sources. 
tree height $(z)+($ error term $)-($ distance between tree base and nearest point on

the line + line height modifier)

By utilizing the error term, the Threat Detection workflow's resulting selection of trees would be larger. Yet, if risk mitigation efforts are applied to the larger number trees, the amount of risk posed to the electrical infrastructure would be conservative. This possibility was not fully explored in this project and is worthy of further research.

\section{Likelihood of Failure}

As we were not able to inspect and estimate the failure potential of each tree using our remote sensing tools, a surrogate measure of each of our lidar derived tree's Likelihood of Failure used the interaction of tree height $(\mathrm{Z})$ and $\mathrm{DBH}$ because height and diameter have been two of the more consistent variables for predicting tree failure (Kane 2008, Peterson 2007, Gardiner et al. 2008, Dahle et al. 2017). Yet, lidar does not directly provide DBH. So, we selected one of our individual tree detection and segmentation results which had matched 28 trees and used it to develop a model to predict the field measure tree DBH from the lidar derived height and crown area. We then predicted the DBHs for the other matching trees from every individual tree crown detection and segmentation result. Once each tree's diameter was predicted from tree height and crown area, that value is multiplied by tree height to obtain a measure for likelihood of failure.

We categorized the likelihood of failure values into categories from the International Society of Arboriculture's tree risk assessment BMPs (Smiley et al. 2017), see Figure 26. We followed an exponential scale for the categories, using the quantiles as guide because, it visually made sense with the distribution of the data, as well as with observational experience of trees. 
While the overall ANOVA on the Kappa values of agreement for the Likelihood of Failure classification compared to the validation classifications were statistically significant $(\mathrm{p}$ value $=0.0022)$, Kappa values only demonstrated moderate agreement and ranged from -0.142 to 0.625, across all methods. Effect tests concluded that the point density (p-value $=0.0073$ ), DEM for point cloud normalization $(\mathrm{p}$ value $=0.0018)$, and the Match Method $(\mathrm{p}$ value $=0.0486)$ had significant effects on the mean value of Kappa.

We found the fact that decimated models outperformed full density models, with an average Kappa of 0.4173 compared to 0.3350 , interesting because one would expect that the full density lidar would have a higher probability of striking the actual treetop, thus resulting in a more accurate and thus, higher measure for tree height. Given that tree height was used to predict DBH and that the two inputs into the calculation of our Likelihood of Failure measure were tree height and DBH, it was expected that full density models would have obtained better Kappa values for the agreement of Likelihood of Failure classification, but that was not the case. Furthermore, lidar has been observed to often underestimate tree height but in this study, we found that tree height was consistently overestimated (Brandtberg et al. 2003, Perrson \& Holmgren 2004, Heurich 2008, and Hyyppa et al. 2004).

Nevertheless, the $2.3 \mathrm{~m}$ matching method performed best in the classification of Likelihood of Failure with an average Kappa of 0.4134 , while the Adaptive method performed the worst with a Kappa of 0.3252 , and the most lenient matching routine, $4.5 \mathrm{~m}$, obtained a Kappa value of 0.3895 . The researchers found these results intriguing because the adaptive matching routine was arguably the best at tree DBH prediction which is a constituent part of the Likelihood of Failure surrogate measure. Perhaps, the adaptive matching method's potential bias 
for tree DBH prediction was balanced by the fact that the method was also debatably the worst at determining tree height.

In the future, a more formalized method of predicting the Likelihood of Individual tree failure from remotely sensed data, such as GALES, HWIND, or FOREOLE, should be deployed in a way to work in concert with the ISA's tree risk assessment BMPs. Indeed, the Utility Tree Risk Assessment BMPs (Goodfellow 2020), released as this thesis was in the final stages of development, were designed to apply the ISA's tree risk assessment BMPs at the population level rather than the individual tree level. Hence the incorporation of models such as GALES, HWIND, or FOREOLE are now even more directly applicable. until one of these models can be implemented in concert with the ISA's tree risk assessment BMPs, our surrogate model offers simple implementation that is supported by arboricultural literature.

\section{Likelihood of Impact}

As with the Threat Detection workflow, our attempt at quantifying the Likelihood of Impact of trees from within an UAS-based lidar point cloud appears to be a first within the literature. For the calculation of the Likelihood of Impact, we utilized the buffer around each lidar derived tree's approximated base location that was equivalent to its height. Then, we summarized the lengths of electric line that fell inside those buffers to provide a scale for the Likelihood of Impact, such that trees possessing a longer length of line inside their buffer could fall at a greater range of degrees and still contact the line, see Figure 31.

In the future, this method for measuring the Likelihood of Impact should be refined further, perhaps actually attempting to calculate the range of angles which could result in impact, because length of line alone may not always accurately approximate the potential range of 
degrees, or angle of potential impacts. Again, see the inset image in Figure 31 for an example of a tree that will have its Likelihood of Impact overestimated based on the current methodology.

All matching methods possessed at least one model which achieved a Kappa value for agreement of 1.0, demonstrating perfect agreement for the Likelihood of Impact classification. models from both the $4.5 \mathrm{~m}$ and the adaptive matching routines demonstrated this perfect agreement and Kappa was determined to be statistically significant. However, none of the models from the $2.3 \mathrm{~m}$ matching routine were deemed significant.

The overall ANOVA of the Kappa values of agreement for the Likelihood of Impact classification compared to validation revealed a statistically significant difference in the means of Kappa values ( $\mathrm{p}$ value $=<0.0001$ ). Effect tests conclude that the DEM for point cloud normalization $(\mathrm{p}$ value $=<0.0001)$, point cloud density $(\mathrm{p}$ value $=0.0140)$, matching method $(\mathrm{p}$ value $=0.0193)$, and the matching feature $(\mathrm{p}$ value $=0.0334)$ had statistically significant effects on the mean value of Kappa.

why TIN models outperformed KNN models, averaging a Kappa value of 0.6833 , compared to 0.3368 , was not determined. Furthermore, the effects of decimation whereby decimated models outperformed Full density models, averaging a Kappa value of 0.5673 , compared to the latter's 0.4160 is also not understood. Nor were we able to determine why adaptive matching method performed best, averaging a Kappa of 0.6139, while $2.3 \mathrm{~m}$ method averaged a Kappa of 0.3311 , and 4.5 m method averaged 0.4751 .

Between the two tree base approximation methods, the $\mathrm{CP}$ (center point of polygon) estimation of tree base location outperformed XY (average x,y of all points in that "treeID"), averaging a Kappa value of 0.5554 , compared to the latter's 0.4146 . Thus, the CP method for 
base estimation more closely aligned with the results of the Likelihood of Impact classification of field trees.

\section{Result of Likelihood Matrix}

Once we had entered values into the positions for the Likelihood of Failure and Likelihood of Impact, we then followed the ISA's pre-existing framework. The results from this section combined the results from the other two likelihood measures into one of four categories from the ISA tree risk assessment BMPs (Smiley et al. 2017) based on our conditional if/then formula, in Figure 34. The distributions of Kappa values for the Likelihood Matrix are available in Figures $52 \& 53$.

Unexpectedly, in all models where Kappa agreement statistics could be calculated, they were significant. Equally unexpected was that over half (5/9) of the significant models in the 2.3 $\mathrm{m}$ matching routine possessed a Kappa of 1.0. Additionally, across all matching routines, agreement was generally better for the Likelihood Matrix output, than it was for either of its two constituent parts, Likelihood of Failure and Likelihood of Impact.

The overall ANOVA of the Kappa values of agreement for the classification results of the Likelihood Matrix demonstrated a statistically significant difference in the means of Kappa values $(\mathrm{p}$ value $=0.0002)$, and the effect tests conclude that the matching method $(<0.0001)$, canopy modeling parameter (0.0031), and the DEM used for point cloud normalization (0.0084) had significant effects on the mean of Kappa values.

The $2.3 \mathrm{~m}$ matching method performed the best with a Kappa value of 0.8258 . The adaptive matching routine outperformed the 4.5 method, obtaining a Kappa value of 0.6670 , compared to the latter's 0.6120 . The reason why the $2.3 \mathrm{~m}$ performed so much better is not clear, we had originally suspected that increased position accuracy within the matching routine could 
have contributed to more accurate Likelihood of Impact classification, thus leading to better Likelihood Matrix results. However, the $2.3 \mathrm{~m}$ matching routine performed the worst at the classification of Likelihood of Impact, so that possibility seems unlikely.

Furthermore, the models using a single canopy modeling parameter outperformed the divided canopy models, obtaining a Kappa value of 0.7326, compared to 0.6612, and TIN DEM normalized models outperformed KNN DEM normalized models, obtaining a Kappa value of 0.7353, compared to the latter's 0.6446 . again, we were unable to attribute causes for the differences.

\section{Consequences of Impact}

By design all methods demonstrated perfect agreement for the classification of the Consequences of Impact. This was necessary to determine how the lidar derived tree metrics effected our threat detection, likelihood of failure, and likelihood of impact analyses. In the future, the Consequences of Impact should be modified to allow for trees which can hit multiple segments of line with different customer counts. This would allow each tree to possess multiple levels of consequences, each with their own Likelihood of Impact. Although, this level of detail within the tree risk assessment frameworks presented here has yet to be accomplished.

\section{Final Risk Rating}

Again, once we had entered values into the positions for the Likelihood of Failure and Likelihood of Impact measures and held the Consequences of Impact model constant, we simply followed the ISA's pre-existing framework. Similar to the results from the Likelihood Matrix before, for all models where Kappa could be calculated it was statistically significant. Although here, out of the models where Kappa could be calculated $(\mathrm{N}=37)$, a quarter of them demonstrated a Kappa of 1.0, see Figures 52 \& 53. 
The results for the Final Risk Rating classification possessed a statistically significant ( $p$ value $=0.0037)$ overall ANOVA for the values of the Kappa agreement statistics. The effect tests concluded that the DEM used for point cloud normalization ( $\mathrm{p}$ value $=<0.0001$ ) had a significant effect on the values of Kappa for the Final Risk Rating classification. TIN normalized methods outperformed the KNN normalized methods, averaging a Kappa value of 0.8562 , compared to the latter's Kappa average of 0.6477 , yet the cause of the difference is not fully understood.

Future research should continue the development of models for the identification of threats and risk assessment of vegetation from within remotely sensed point clouds. Additionally, further work is needed to completely adapt the ISA's tree risk assessment framework to utility vegetation management from remote sensing technologies, particularly since the ISA recently released new, population-based utility tree risk assessment best management (Goodfellow 2020).

\section{Tree Lean}

This study had initially intended to assess tree lean from lidar. Yet, this became impractical, primarily during the field data collection, as no single method was identified as the best method for the assessment of tree lean. Ko et al. (2012) described a method for determining whether trees were leaning towards or away from the powerlines, but neither their tree base information nor the direction of lean analysis included an accuracy assessment of their results. Future research should be devoted to tree lean measurement both for field data collection and from lidar. This would allow for the accuracy assessment of lidar-based lean measures via comparison to field-collected lean measures. If lidar based measures are determined to be accurate then, the ability to assess whether trees are leaning towards or away from electrical 
infrastructure would be possible and the assessment would play a pivotal role in the assessment of tree risk presented to electrical infrastructure.

\section{Model Selection}

Since the primary goal of the study was the detection, matching, and risk assessment of NERC category $2 \& 3$ trees, we decided to base part of our model selection upon the percentage of properly identified NERC category $2 \& 3$ vegetation threats. Our best model based upon this metric was the TIN DEM normalized, decimated, divided canopy model where tree bases were approximated using the XY method and matched using the $4.5 \mathrm{~m}$ matching routine. This model possessed the best ratio of Esyn et al.'s (2015) accuracy index for models not using the treetops matching feature. This model also performed the best of those included in the Threat Detection protocol and possessed the best rate of identification of vegetation threats in the study.

Additionally, the model correctly identified seven of the 14 vegetation threats determined by the field data's Threat Detection results, the highest percentage of trees correctly detected, segmented, matched, and classified by Threat Detection, in the study. See Figure 59, for the distribution of models by the percentage of field threats which were correctly detected, segmented, matched, and classified by Threat Detection. The model matched a total of 41 trees in our field plots. All classification errors for Threat Detection were False Positives, which is preferable to False Negatives in our situation. The contingency analysis for this method's Threat Detection classification can be seen in Figure 60. 


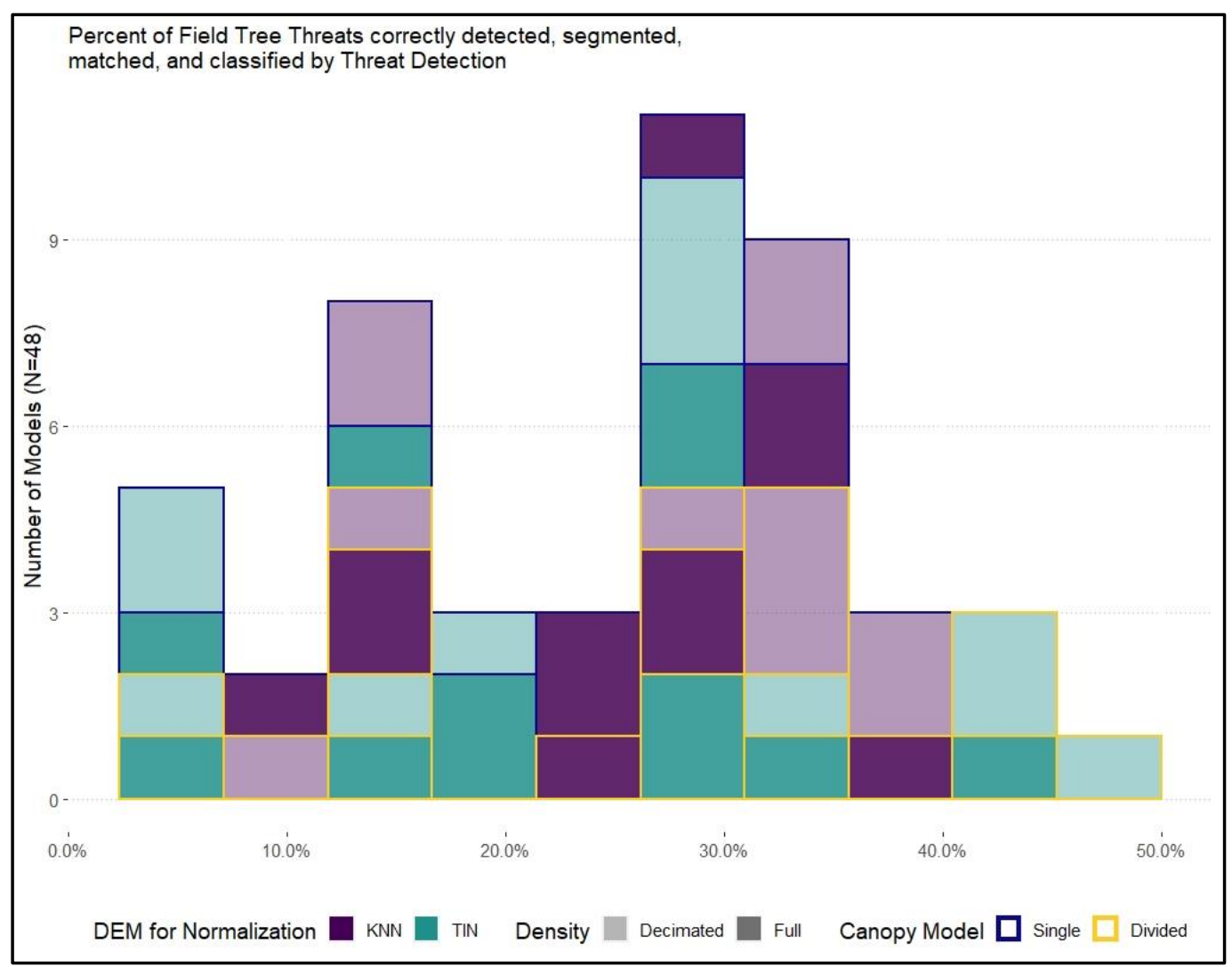

Figure 59: Percent of Field Tree Threats correctly detected, segmented, matched, and classified by Threat Detection. 


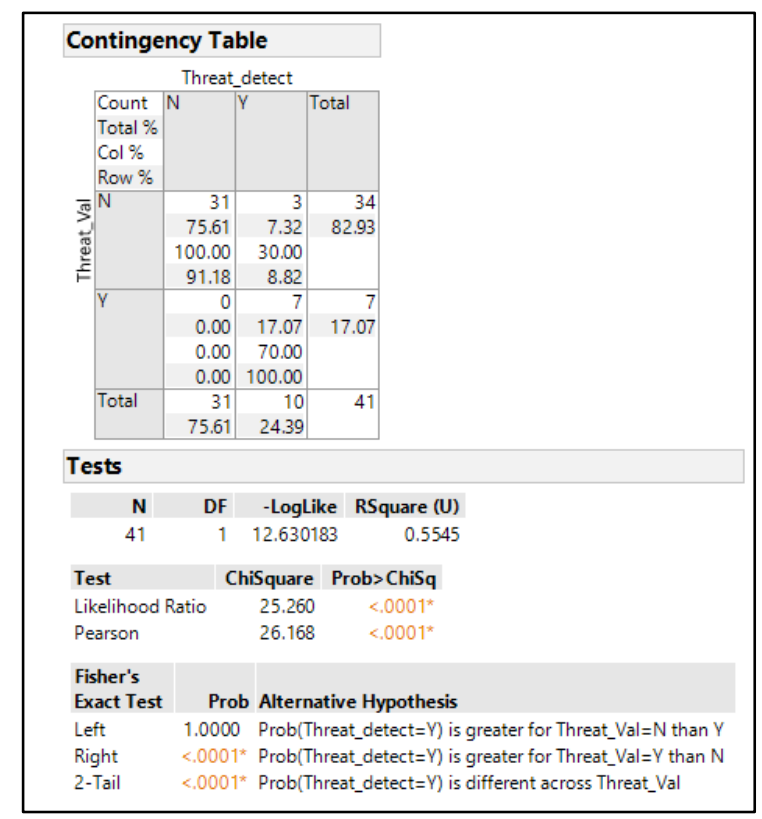

Figure 60: Contingency analysis for the TIN DEM normalized, decimated, divided canopy model, using the XY tree base approximation method and the $4.5 \mathrm{~m}$ matching method, which was selected for Threat Detection performance

we fitted percentage of field threats which were correctly detected, segmented, matched, and classified by Threat Detection to the tree matching rate, see Figure 61. The two variables exhibited an $\mathrm{R}^{2}$ value 0.519751 and an ANOVA revealed a significant difference in threat detection associated with the tree matching rate (p-value: $<0.0001)$. The parameter estimates for the intercept and tree matching rate were both significant (p-values: $<0.0001)$. These parameter estimates were used to construct the prediction equation:

\section{$0.1110+1.3247 *$ Tree Matching Rate}

We set 'Tree Matching Rate' as ' $x$ ' and the equation equal to ' 1.0 ' and solved for ' $x$ ' resulting in the value 0.671017186 or the expected Match Rate at which all vegetation threats will be identified by the methods used in this study. 


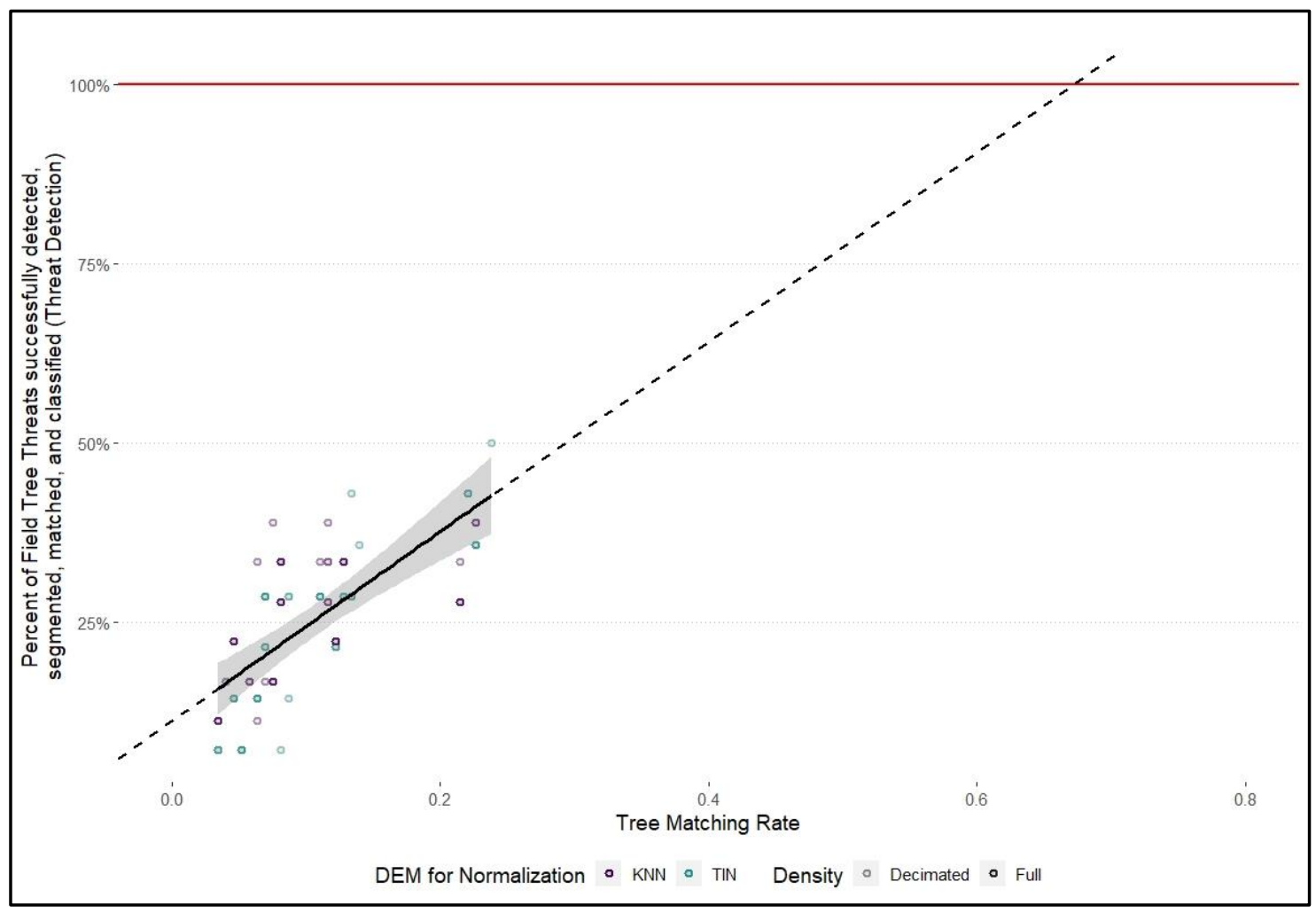

Figure 61: Projected tree matching rate necessary to correctly detect, segment, match, and classify all Field Threats

Lastly, it should be noted that a tree matching rate of 0.67 is high, but not unheard of within the literature (Wallace et al. 2014, Pirotti 2010, Eysn et al. 2015, Vauhkonen et al. 2012, Kaartinen et al. 2012). examples of studies where greater tree matching rates have been achieved generally occur in regularly structured plantations, or more homogenously structured forests; not in mixed forest settings comparable to those described in this study (Wallace et al. 2014).

Additionally, we decided to include a description of the model which was determined to be the best for individual tree detection and segmentation, which was practically the same model used for the identification of vegetation threats. The only difference between the two models was the matching feature. Where our best model for individual tree detection and segmentation used 
the treetops matching feature, rather than the XY tree base approximation. This model matched the most trees (44) of any model but due to the using the matching feature of tops, no base location was determined for the trees, and thus these trees were not used within the Threat Detection and tree risk assessment frameworks. This models individual tree detection and segmentation results can be seen in Figure 18. In the future, the treetops matching feature should be used for the matching routines after individual tree detection and segmentation. Then, after the matching is complete, tree base location should be estimated from within those treetop-based matches, thus allowing for higher matching rates.

Furthermore, we determined the best model for the adaptation of the International Society of Arboriculture's tree risk assessment BMP classification process by looking at the Kappa agreement performance within each of the risk assessment components as well as looking across the components. We determined that the model, which was TIN DEM normalized, full density, with divided canopy types, utilizing the $\mathrm{CP}$ base approximation method as the matching feature, within the $2.3 \mathrm{~m}$ matching routine, performed best. This model was tied for the highest Kappa value in Likelihood of Impact, Likelihood Matrix, and Final Risk Rating classifications. Additionally, this model possessed the highest average Kappa across the classifications, with a value of 0.8281 . Yet, this method only correctly matched 11 trees in total. Only two of which were classified as trees deemed vegetation threats by the field data.

Furthermore, looking across our selected models' share the use of the TIN DEM for normalization and divided canopy types, which suggests some level of stability in the accuracy of tree detection and segmentation routines. Future research should continue the development of models for the identification vegetation threats and risk assessment of vegetation from within 
remotely sensed point clouds. further work is also needed to completely adapt the ISA's tree risk assessment framework to utility vegetation management from remote sensing technologies.

\section{Discussion of the Project's Goals}

The goals of this study were to: (1) examine how Digital Elevation Model (DEM) interpolation methods effect individual tree crown detection and segmentation routines and tree risk analysis from high-density lidar, (2) determine the effect of point cloud decimation on individual tree crown detection and segmentation routines and tree risk analysis, (3) develop a tree base location approximation method for the identification of NERC Category $2 \& 3$ vegetation risks, and (4) develop a methodology to estimate the Likelihood of Impact of a NERC Category $2 \& 3$, "fall-in" vegetation risks on the electric lines.

The KNN and TIN DEMs used throughout our study were generated using lidR's "grid_terrain" function with default parameters for both methods. The two were compared to elevations obtained using the Spectra SP80 GPS, and their mean errors were roughly the same at $1.4884 \mathrm{~m}$ and $1.5896 \mathrm{~m}$, respectively. Despite the slightly larger error, the TIN DEM appears better at the approximation of tree base elevations. The DEM for normalization was found to be significant source of variance within the $2.3 \mathrm{~m}$ and adaptive matching routines. for this comparison, field trees' elevations were extracted from the same lidar derived DEMs from which the lidar derived trees' bases were extracted.

TIN DEM normalized methods performed better in Likelihood of Impact agreement, Likelihood Matrix agreement, and Final Risk Rating agreement. KNN was only found to be 
better at the agreement of Likelihood of Failure. Thus, in this study, TIN DEM normalized models generally performed better, but this cannot be expected to always be the case.

The effect of decimation on individual tree detection and segmentation routines and risk analyses was limited in this study, which seems to suggest that a point density of roughly 50 $\mathrm{ppm}^{2}$ is sufficiently dense for the accurate description of detected and segmented tree properties, such as height, crown area, diameter at breast height. Within the individual detection and segmentation framework presented here, the $2.3 \mathrm{~m}$ matching routine demonstrated an effect due to decimation, where the full density models performed marginally better than the models decimated to $50 \mathrm{ppm}^{2}$ for the estimation of tree base location, which concurs with the literature (Wallace et al. 2014). Yet, this effect was limited to only the $2.3 \mathrm{~m}$ matching routine. Within our risk assessment framework, decimated models performed better at Likelihood of Failure and Likelihood of Impact classifications according to Kappa agreement statistics, although these results are not fully understood.

Our base approximation methods performance within tree detection and segmentation metrics were not identified as significant by any ANOVA. Furthermore, within the risk assessment framework, only once was the matching feature determined to be a significant source of variance. This one instance occurred during the Likelihood of Impact classification, and the CP base approximation methods outperformed the XY method's models. For future work, base location approximations should continue to be developed, particularly for the further refinement of the Threat Detection process. Yet, until the base approximations can perform comparably to the treetops as a matching feature, treetops should be used for tree matching routines as this feature clearly outperformed the tree base approximations both within and across the matching 
routines as determined by the matching rate, Precision, Recall, F-score, and Commission Error see Figures 46 \& 47.

this study demonstrated a methodology for the identification and risk assessment of NERC Category $2 \& 3$ "fall-in" vegetation threats. our models did not perform well enough to be utilized in practice, as our best model, the TIN DEM normalized, decimated, divided canopy model where tree bases were approximated using the XY method and matched using the $4.5 \mathrm{~m}$ matching routine, was only able to identify half of the trees determined to be threats by our field data, see Figure 59. Of this model's detected threats, all were in the "Low" or "Moderate" categories and it missed three of the four threats with a Final Risk Rating above "Low" in TIN DEM Threat Detection using field tree heights and location, see Figure 43. Yet, also worthy of note is that this model's Threat Detection results demonstrated correct classification for all seven of the matched threats and only two False Positive results, see Figure 59.

Given that our overall tree detection rates included models with $>100 \%$ detection and appear comparable to the literature, the author is of the opinion that the core issue of the Threat Detection workflow performance in this study are the poor matching rates, see Figure 46 (Wallace et al 2014, Pirotti 2010, Eysn et al. 2015, Vauhkonen et al. 2012). Despite the poor matching rates, we were capable of the correct detection, segmentation, matching, and Threat Detection classification of 50\% of field trees which were deemed vegetation threats.

Additionally, we suggest that, at roughly a tree matching rate of 70\%, all NERC Category $2 \& 3$ vegetation threats will be capable of being correctly detected, segmented, matched, and classified by Threat Detection, see Figure 61. Furthermore, literature has demonstrated examples of tree matching rates of $40-60 \%$ fairly consistently, but not many examples exist of matching 
rates higher, particularly in mixed forest settings (Wallace et al 2014, Pirotti 2010, Eysn et al. 2015, Vauhkonen et al. 2012, Kaartinen et al. 2012).

Given that the Likelihood of Failure demonstrated here is a surrogate measure, the accuracy of this classification doesn't hold much meaning. Yet, given that the Likelihood of Impact measure was calculated in a way which attempts to quantify the actual Likelihood of Impact the author does consider the accuracy of this classification meaningful. Furthermore, the model which provided the best Threat Detection results, the TIN DEM normalized, decimated, divided canopy model where tree bases were approximated using the XY method and matched using the $4.5 \mathrm{~m}$ matching routine, also possessed Kappa of 0.6 for the Likelihood of Impact classification and both examples of misclassification were overestimations of the Likelihood of Impact. This model also correctly assigned a Final Risk Rating for all but one of its 41 matched trees where, again, the model overestimated the Final Risk Rating of one tree. Sadly, only one of this model's seven matched and correctly classified vegetation threats possessed a Final Risk Rating higher than "Low", and this tree was classified as "Moderate". Thus, one tree in each class above "Low" was unmatched within this model's field tree classification dataset.

Despite these challenges, we found the results hold much promise for future remotely sensed threat detection and tree risk assessment frameworks. Future efforts should be focused on obtaining higher matching rates to determine if Threat Detection and tree risk assessment accuracy metrics are stable once a greater number of trees have been matched. Furthermore, the incorporation of site variables, such as soil type, proximity to water, and/or wind patterns, could allow for improved estimates of the Likelihood of Failure. Coupling these variables with models such as GALES, HWIND, or FOREOLE, should allow the incorporation of population level tree risk assessment in utility corridors as described in the new Utility tree risk assessment BMPs. 
the results were expected to show that a proportion of trees near distribution lines could come into contact with the lines, due to ROW width when compared to tree heights. The results from our field data's Threat Detection process shows that $8-10 \%$ of the field surveyed trees were identified as either a NERC Category 2 or 3 vegetation risk. the model we selected as the best for Threat Detection, found a total of 74 trees in the entire AOI $(\mathrm{N}=1091$, total detected and segmented crowns) to be NERC Category $2 \& 3$ or "fall-in" threats. while this model performed the best at Threat Detection classification, it only positively matched $50 \%$ of the trees determined to be field threats. Thus, our lidar -based estimate for the percentage of NERC Category 2 or 3 threats within the population of the AOI is $7-14 \%$. Over the entire length of a distribution circuit or the entire grid, if $7-14 \%$ of trees are NERC Category 2 or 3 vegetation risks, this would mean that a substantial portion of trees along ROWs pose a threat to the electrical infrastructure.

\subsection{Conclusion}

the majority of unplanned electric power disruptions occur on the distribution system, with vegetation-related outages generally regarded as the leading cause (Guggenmoos, 2003, 2007; Ituen et al. 2008). Of these tree-caused or related unplanned disruptions, most are attributed to "fall-ins", trees which were tall enough to fall into the ROW and across or through the electric line. NERC FAC-003 classifies these "fall-ins" into two categories by base location where on-ROW fall-ins are Category 2 vegetation threats and off-ROW fall-ins are Category 3 vegetation threats. While we utilized the existing NERC vegetation threat classes for this research project exploring the use of UAS and lidar for distribution utility vegetation management, the authors are not suggesting that NERC FAC-003 standards be applied to 
distribution UVM. However, the NERC FAC-003 vegetation threat classification system was a convenient categorization for this remote sensing study.

This study addressed a research gap specific to the application of UAS-based lidar for distribution level utility vegetation management. A high-density UAS-lidar point cloud was acquired of a distribution electric ROW in north central WV. The point cloud was semi-manually classified before $0.5 \mathrm{~m}$ DEMs were generated. These DEMs were used to normalize the point cloud before multiple implementations of the individual tree detection and segmentation algorithm described in Li et al. (2012) was used to identify and segment trees.

Once the trees were detected and segmented, we utilized a series of spatially dependent calculations to determine if a tree could contact the electrical infrastructure should the tree experience failure. We called this our Threat Detection workflow. a surrogate measure for the Likelihood of Failure was developed based upon arboricultural post-storm studies' observations of tree failure. The Likelihood of Failure measure was then used to classify trees into the Likelihood of Failure categories presented in the International Society of Arboriculture's tree risk assessment Best Management Practices (Smiley et al. 2017). Then, these lidar -derived NERC category 2 and 3 vegetation threats were buffered by their height and the length of electrical conductor inside that buffer was used as a measure of the Likelihood of Impact. This measure was used to classify trees into ISA's Likelihood of Impact categories. Once the two measures for Likelihood of Failure and Likelihood of Impact were classified, we followed the ISA's existing framework, while using a single model for the Consequences of Impact across models. Finally, the tree crown polygons for the TIN DEM normalized, decimated, divided canopy model where tree bases were approximated using the XY method and matched using the $4.5 \mathrm{~m}$ matching routine, was displayed as a series of choropleth maps indicating their 
classification results for the Threat Detection, Likelihood of Failure, Likelihood of Impact, and the Final Risk Rating, see Figures 25, 28, 29, 32, 33, 38, and 39.

During this study, we assessed how DEM interpolation method and point cloud decimation affected individual tree crown detection and segmentation, the identification of NERC Category $2 \& 3$ vegetation threats, and the classification of trees into the ISAs tree risk assessment Likelihood of Failure, Likelihood of Impact, and Final Risk Rating categories. We found that during the individual tree detection and segmentation processes, the DEM for used for point cloud normalization had a significant effect on the difference of matched field and lidar derived trees' base elevations, where the TIN DEM was superior. While during the Risk Analysis, the DEM used for point cloud normalization contributed to significant differences in the Kappa values for Likelihood of Failure, Likelihood of Impact, Likelihood Matrix, and Final Risk Rating classifications. TIN models outperformed KNN models in every classification except for the Likelihood of Failure classification.

We also examined the effect of point cloud decimation on individual tree crown detection and segmentation routines and tree risk analysis. During the individual tree detection and segmentation routines, point density was found to have a significant effect on the differences of tree base locations within the $2.3 \mathrm{~m}$ matching routine from Pirotti (2010) where, full density models outperformed decimated models. Yet, the increase in accuracy was of only $0.2 \mathrm{~m}$, and similar to Wallace et al. (2014), such a minimal increase in accuracy may not be worth the increase in processing time. Point cloud density was also found to be a source of significant variation in the Kappa values of Likelihood of Failure and Likelihood of Impact classifications and, in both cases decimated models outperformed full density models. 
Given that our lidar -derived tree inventory did not directly supply each tree's base location, we developed two methods for the approximation of lidar derived tree bases. The first, the "XY" method, utilized the averaged the $\mathrm{x}, \mathrm{y}$ coordinates for all points within each tree ID. For the second, the "CP" method, the tree base was placed at the center of each tree crown polygon. These approximated tree base locations were used as a matching feature along with the lidar derived treetops within each of our three matching routines. The treetops feature matching routine clearly outperformed the two base approximations, providing the best measures of precision, recall, F-score, and commission error, with only a few exceptions limited to the $2.3 \mathrm{~m}$ matching regiment, see Figure 47. only during the ANOVA of Likelihood of Impact classification Kappa values was the matching feature a significant source of variability, and the CP method outperformed the XY method.

We also sought to develop a methodology to estimate the Likelihood of Impact of NERC Category $2 \& 3$, "fall-in" vegetation risks on the electric lines. The methodology we developed first detected these threats within our Threat Detection workflow. Then, using each tree's base location, height, and elevation and the lines' location, height, and elevation, we calculated the length of line that was inside each tree's potential fall zone. This length of line was used to provide scale for the Likelihood of Impact variable and to differentiate the different levels of our Likelihood of Impact classification. Furthermore, the models included in the Risk Analysis averaged a Kappa value of 0.7273 for the Threat Detection classification $(\mathrm{N}=48)$ and 0.4899 for the Likelihood of Impact classification $(\mathrm{N}=43)$.

In addition, we implemented and examined three different tree matching distances, two from the literature, Pirotti's (2010) 2.3 m matching distance and Esyn et al.'s (2015) height adaptive matching distance, as well as a $4.5 \mathrm{~m}$ matching distance which we constructed. We 
concluded that as the matching method becomes less stringent in the precision of the match, the matching rate increases, but at the expense of tree location accuracy. We also investigated a notion put forward by Larsen et al. (2011) of splitting scenes before individual tree detection and segmentation by stand type. We manually divided our AOI and point clouds by the broad categories, "open canopy" and "closed canopy". Then, we ran individual tree detection and segmentation routines tailored to each, before merging the datasets for comparison with the results of single canopy, undivided point cloud tree detection and segmentation results. We found that the divided canopy models excelled at the estimation of tree height within the $2.3 \mathrm{~m}$ matching routine, and also possessed a trend towards better performance at DBH prediction within this matching routine. Yet, the single canopy models possessed higher average Kappa agreement statistics for Threat Detection and the Likelihood Matrix classifications. the higher Kappa values were offset by the single canopy models possession of lower rates of trees correctly detected, segmented, matched, and classified by Threat Detection.

The ultimate goal of this project was to develop a methodology to identify all NERC Category 2 and 3 vegetation threats and assess the risk of each to electrical infrastructure, with an UAS-based lidar system. The study assessed the effect of DEM interpolation methods and the effect of point cloud decimation on individual tree crown detection and segmentation and tree risk assessment frameworks. Additionally, we developed a pair of methods for tree base location approximation and assessed effect of these tree base location approximation methods on individual tree detection and segmentation as well as, the identification of NERC Category 2 and 3 vegetation risks. We also developed a method to estimate the Likelihood of Impact of NERC Category 2 and 3 vegetation risks on the electrical infrastructure. We then assessed the agreement between the Likelihood of Failure classifications of field and lidar -derived trees. 
Furthermore, we demonstrated a simplified adaptation of the International Society of Arboriculture's tree risk assessment BMP method using lidar derived tree properties.

the models presented here may serve as recommendations for where to focus vegetation management activities to most effectively mitigate NERC Category $2 \& 3$ vegetation risks to electrical infrastructure. The intended result of these efforts is fewer tree-caused outages and improvements in electrical reliability and grid stability. We adapted the International Society of Arboriculture's tree risk assessment best management practices to derive the risk presented to electrical infrastructure by the NERC Category $2 \& 3$ vegetation threats from individual tree detection and segmentation results from within an UAS-lidar point cloud. We suggest that the methodology presented here should be improved to incorporate the newly released utility tree risk assessment best management practices for risk evaluation at the population level. The assessment included the development of a stand in Likelihood of Failure measure in lieu of the actual Likelihood of Failure and, featured an attempt to quantify the actual Likelihood of Impact of each of the identified NERC Category 2 and 3 vegetation threats. Our series of maps based upon the model selected as the best for Threat Detection is the final deliverable of this project, provided in Figures 25, 28, 29, 32, 33, 38, and 39. 


\section{References}

Achim A, Wellpott A, Gardiner B. 2007. Competition Indices as a Measure of Wind Loading on Individual Trees, 2007 International Conference on Wind and Trees, 5-9 August 2007, University of British Columbia, Vancouver, Canada

Albers, J., Hayes, E. 1993. How to detect, assess and correct hazard trees in recreational areas. Minnesota Department of Natural Resources Publication, 64 P.

Ancelin P, Courbaud B, Fourcaud T. 2004. Development of an individual tree-based mechanical model to predict wind damage within forest stands, For. Ecol. Manage., vol. 203

Appelt, P.J., Goodfellow, J.W. 2004. Research on how trees cause interruptions- Applications to Vegetation Management. IEEE Rural Electric Power Conference. Paper No. 04 C6.

Asner, G.P. \& Goldstein, G. 1997. Correlating stem biomechanical properties of Hawaiian canopy trees with hurricane wind damage. Biotropica, 29, 145- 150.

Baker, CJ. 1995. The development of a theoretical model for the windthrow of plants. J Theor Biol 175:355-375.

Ball, DJ. 2007. The evolution of risk assessment and risk management: a background to the development of risk philosophy. Arboricultural Journal, 30, 105-112.

Ballard, B.D., McLoughlin, K.T. Nowak, C.A. 2007. New diagrams and applications for the wire zone-border zone approach to vegetation management on electric transmission line rightsof-way. Arboriculture \& Urban Forestry, 33(6), 435-439.

Bodig, J., and B.A. Jayne. 1982. Mechanics of Wood and Wood Composites. Van Nostrand Reinhold Company, New York, U.S

Bramble, W.C., W.R. Byrnes, M.D. Schuler. 1986. Effects of a special technique for right-ofway maintenance on an avian population. Journal of Arboriculture 12: 219-226.

Bramble, W.C., W.R. Byrnes, R.J. Hutnik. 1985. Effects of a special technique for right-of-way maintenance on deer habitat. Journal of Arboriculture 11:278-284.

Brandtberg, T., Warner, T. A., Landenberger, R. E., \& McGraw, J. B. (2003). Detection and analysis of individual leaf-off tree crowns in small footprint, high sampling density lidar data from the eastern deciduous forest in North America. Remote Sensing of Environment, 85(3), 290-303. https://doi.org/10.1016/S0034-4257(03)00008-7

Brunker, S. W. (2016). Aerial LiDAR for overhead transmission lines-Lessons from the USA in realising benefit and value. 2016 IEEE International Conference on Power System Technology, POWERCON 2016, 1-6. https://doi.org/10.1109/POWERCON.2016.7753929

Burgert, I. 2006. Exploring the micromechanical design of plant cell walls. American Journal of Botany 93:1391-1401. 
Burgert, I. 2006. Exploring the micromechanical design of plant cell walls. American Journal of Botany 93: 1391- 1402.

Canham, C.D. and Loucks, O.L. 1984. Catastrophic Windthrow in the Presettlement Forests of Wisconsin. Ecology, 65: 803-809.

Carson, W. W., Andersen, H.-E., Reutebuch, S. E., \& Mcgaughey, R. J. (2004). LIDAR APPLICATIONS IN FORESTRY - AN OVERVIEW. In ASPRS Annual Conference Proceedings.

Ciftci, C., Arwade, S., Kane, B., \& Brena, S. 2014. Analysis of the probability of failure for open-grown trees during wind storms. Probabilistic Engineering Mechanics 37, 41-50.

Ciftci, C., Kane, B., Brena, S., \& Arwade, S. (2013). Loss in moment capacity of tree stems induced by decay. Trees.

Clair B., M. Fournier, M.F. Prevost, J. Beauchene, and S. Bardet. 2003. Biomechanics of buttressed trees: Bending strains and stresses. American Journal of Botany 90:1349-1356.

Clough, R.W., and J. Penzien. 1993. Dynamics of structures. McGraw Hill, New York, City, New York, U.S

Coutts, M.P. 1986. Components of tree stability in Sitka spruce on peaty gley soil. Forestry 59:173-197.

Crook, M.J., and A.R. Ennos. 1996. The anchorage mechanics of deep rooted larch, Larix europea $\times$ L. japonica. Journal of Experimental Botany 47:1509-1517.

Cullen, S. 2002b. Trees and Wind; Wind scales and speeds. Journal of Arboriculture 28(5):237242.

Dahle G.A., Grabosky J.C. 2009. Review of literature on the function and allometric relationships of tree stems and branches. Arboricult Urban For 35(6):311-320

Dahle, G., Grabowsky, J., Kane, B., Misebauer, J., Peterson, W., Telewski, F., Koeser, A., \& Watson, G. (2014). Tree Biomechanics: A white paper from the 2010 international meeting and research summit at The Morton Arboretum. Arboriculture \& Urban Forestry 40(6), 309-318.

Dahle, G.A., and J.C. Grabosky. 2010a. Allometric patterns in Acer platanoides (Aceraceae) branches. Trees: Structure and Function 24:321-326

Dahle, G.A., and J.C. Grabosky. 2010b. Variation in modulus of elasticity (E) along Acer platanoides L. (Aceraceae) branches. Urban Forestry \& Urban Greening 9:227-233.

Dahle, G.A., and J.C. Grabowsky. 2012. Determining if lateral imbalance exists in first-order branches leading to a potential development of torsional stress. Journal of Arboriculture \& Urban Forestry 38(4):141-145. 
Dahle, G.A., Grabosky, J.C., Kane, B., Misebauer, J., Peterson, W., Telewski, F.W., Koeser, A.K., Watson, G.W. 2014. Tree Biomechanics: A white paper from the 2010 international meeting and research summit at The Morton Arboretum. Arboriculture \& Urban Forestry, 40(6), 309-318.

Dahle, G.A., H.H. Holt, W.R. Chaney, T.M. Whalen, D.L. Cassens, R. Gazo, and R.L. McKenzie. 2006. Branch strength loss implications for silver maple (Acer saccharinum) converted from round-over to V-trim. Arboriculture \& Urban Forestry 32:148-154.

Dahle, G.A., James, K.R., Kane, B., Grabosky, J.C., Detter, A. 2017. A review of factors that Affect the static load-bearing capacity of urban trees. Arboriculture \& Urban Forestry, 43(3), 89-106.

de Langre, E. 2008. Effects of wind on plants. Annual Review of Fluid Mechanics 40:141-168.

Den Hartog, J.P. 1956. Mechanical Vibrations. McGraw-Hill, New York City, New York, U.S.

Detter, A., and S. Rust. 2013. Aktuelle Untersuchungsergebnisse zu Zugversuchen [Latest results in research on pulling tests] In: D. Dujesiefken (Ed.). Jahrbuch der Baumpflege 2013.

Dunster, J.A., Smiley, E.T., Matheny, N., Lilly, S. 2017. Tree Risk Assessment Manual. International Society of Arboriculture.

Dunster, J.A., Smiley, E.T., Matheny, N., Lilly, S., 2013. Tree Risk Assessment Manual. International Society of Arboriculture, Champaign.

Dupuy, L., T. Fourcaud, P. Lac, and A. Stokes. 2007. A generic 3D finite element model of tree anchorage integrating soil mechanics and real root system architecture. American Journal of Botany 94(9):1506-1514.

Duryea, M. L., Kampf, E., Littell, R. C., 2007. Hurricanes and the urban forest: I. Effects on southeastern United States coastal plain tree species. Arboriculture and Urban Forestry. 33(2), 83-97.

Duryea, M.L., E. Kampf, and R.C. Little. 2007a. Hurricanes and the urban forest I: Effects on Southeastern United States Coastal Plain Tree Species. Arboriculture \& Urban Forestry 33:83-97.

Duryea, M.L., E. Kampf, R.C. Littel, and C.D. Rodriguez-Pedraza. 2007b. Hurricanes and the urban forest II: Effects on tropical and subtropical tree species. Arboriculture \& Urban Forestry 33:98-112.

Ellison, M.J. 2005. Quantified Tree Risk Assessment used in the management of amenity trees. Journal of Arboriculture, 31, 57-65.

Energy. (2017). Retrieved from https://www.infrastructurereportcard.org/cat-item/energy/ 
England, A.H., C.J. Baker, and S.E.T. Saunderson. 2000. A dynamic analysis of windthrow of trees. Forestry 73(3):225-237.

Eysn, L.; Hollaus, M.; Lindberg, E.; Berger, F.; Monnet, J.-M.; Dalponte, M.; Kobal, M.; Pellegrini, M.; Lingua, E.; Mongus, D.; Pfeifer, N. A Benchmark of Lidar-Based Single Tree Detection Methods Using Heterogeneous Forest Data from the Alpine Space. Forests 2015, 6, 1721-1747.

Ferguson, N., Ryder, S., \& Richardson P. 2012. Mitigating the environmental impact of compliance with NERC FAC-003-1: A working methodology for intelligent vegetation hazard detection using aerial lidar technology. Environmental Concerns in Rights-of-Way Management $9^{\text {th }}$ International Symposium. International Society of Arboriculture. Champaign, Illinois.

Francis, J. \& Gillespie, A. (1993). Relating gust speed to tree damage in hurricane Hugo, 1989. Journal of Arboriculture 19(6), 368-373.

Frank, M., Pan, Z., Raber, B., \& Lenart, C. (2010). Vegetation management of utility corridors using high-resolution hyperspectral imaging and LIDAR. In 2nd Workshop on Hyperspectral Image and Signal Processing: Evolution in Remote Sensing, WHISPERS 2010 - Workshop Program. https://doi.org/10.1109/WHISPERS.2010.5594887

Gaffrey, D. \& Kniemeyer, O. (2002) The elasto-mechanical behaviour of Douglas fire, its sensitivity to tree-specific properties, wind and snow loads, and implications for stability - a simulation study. Journal of Forest Science 48(2), 49-69.

Gardiner B, Peltola H, Kellomäki S. 2000. Comparison of two models for predicting the critical wind speeds required to damage coniferous trees, Ecol. Model., vol. 129

Gardiner, B., Byrne, K., Hale, S., Kamimura, K., Mitchell, S., Peltola, H., \& Ruel, J. (2008). A review of mechanistic modelling of wind damage risk to forests. Forestry 81(3), 447-463.

Ghani, M.A., A. Stokes, and T. Fourcaud. 2009. The effect of root architecture and root loss through trenching on the anchorage of tropical urban trees (Eugenia grandis Wight). Trees 23:197-209

Goodfellow, J.W. 2020. Utility Tree Risk Assessment - Best Management Practices. International Society of Arboriculture. Champaign, Illinois, U.S.

Greenhill, A.G. 1881. Determination of the greatest height consistent with stability that a vertical pole or mast can be made, and of the greatest height to which a tree of given proportions can grow. Proceedings of the Cambridge Philosophical Society 4:65-73.

Guggenmoos, S. \& Sullivan, T.E. 2012. Outside-ROW tree risk along electrical transmission lines. Environmental Concerns in Rights-of-Way Management $9^{\text {th }}$ International Symposium. International Society of Arboriculture. Champaign, Illinois.

Guggenmoos, S. (2003). Effects of tree mortality on power line security. Journal of 
Arboriculture, 29(4), 181-192. https://doi.org/S0166-6851(09)00219-9 [pii]

10.1016/j.molbiopara.2009.09.004 [doi]

Guggenmoos, S. (2007). Storm Hardening the Electric System Against Tree-caused Service Interruptions, 1-5.

Guggenmoos, S. 2000. Introducing a new VM tool \& service, optimal clear width calculator. Clubweb.interbaun.com/ecosync/OCWC.htm.

Guggenmoos, S. 2011. Tree-related outages due to wind loading. Arboriculture \& Urban Greening, 37(4), 147-151.

Guggenmoos, S., T.E. Sullivan. 2007. Outside Right-of-Way Tree Risk Along Electrical Transmission Lines. Utility Arborist Association. Mar. 2007.

Guikema, S., Davidson, R., \& Liu, H. (2006). Statistical models of the effects of tree trimming on power system outages. IEEE Transactions on Power Delivery 21(3), 1549-1557.

Hagan, G., \& Smith, J.L. 1986. Predicting tree groundline diameter from crown measurements made on 35-mm aerial photography. Photogrammetric engineering and Remote Sensing. 52(5):687-690.

Hale, S., B. Gardiner, A. Wellpott, B. Nicoll, and A. Achim. 2010. Wind loading of trees: Influence of tree size and competition. European Journal of Forest Research 131(1):203217.

Hamraz, H., Contreras, M. A., \& Zhang, J. (2016). A robust approach for tree segmentation in deciduous forests using small-footprint airborne LiDAR data. International Journal of Applied Earth Observation and Geoinformation, 52, 532-541. https://doi.org/10.1016/j.jag.2016.07.006

Haygreen, J.G., and J.L Bowyer. 1982. Forest Products and Wood Science. Iowa State University Press, Ames, Iowa, U.S. 495 pp.

Helliwell, D.R. 1990. Acceptable level of risk associated with trees. Arboriculture Journal, 14(2), $159-162$.

Heurich, M. (2008). Automatic recognition and measurement of single trees based on data from airborne laser scanning over the richly structured natural forests of the Bavarian Forest National Park. Forest Ecology \& Management. 255. 2416-2433.

Holmes, J.D. 2007. Wind Loading on Structures. 2nd edition. Taylor and Francis, New York City, New York, U.S. 380 pp.

Holmgren, J., Persson, Å., \& Söderman, U. (2008). Species identification of individual trees by combining high resolution LiDAR data with multi-spectral images. International Journal of Remote Sensing, 29(5), 1537-1552. https://doi.org/10.1080/01431160701736471 
Hyyppä' J., Hannu Hyyppä , Paula Litkey, Xiaowei Yu, Henrik Haggrén, Petri Rönnholm, Ulla Pyysalo, Juho Pitkänen, and Matti Maltamo. (2004). Algorithms and methods of airborne laser scanning for forest measurements. International Archives of Photogrammetry, Remote Sensing, and Spatial Information Sciences. Vol. XXXVI.

Ituen, I., Sohn, G., \& Jenkins, A. (2008). A CASE STUDY: WORKFLOW ANALYSIS OF POWERLINE SYSTEMS FOR RISK MANAGEMENT. In The International Archives of the Photogrammetry, Remote Sensing and Spatial Information Sciences.

J. W. Goodfellow, "Applying reliability centered maintenance (RCM) to overhead electric utility distribution systems," 2000 Power Engineering Society Summer Meeting (Cat. No.00CH37134), Seattle, WA, 2000, pp. 566-569 vol. 1, doi: 10.1109/PESS.2000.867647.

Jaakkola, A., Hyyppä, J., Kukko, A., Yu, X., Kaartinen, H., Lehtomäki, M., \& Lin, Y. (2010). A low-cost multi-sensoral mobile mapping system and its feasibility for tree measurements. ISPRS Journal of Photogrammetry and Remote Sensing, 65(6), 514-522. https://doi.org/10.1016/j.isprsjprs.2010.08.002

James, K., Dahle, G., Grabosky, J., Kane, B., \& Detter, A. (2014). Tree Biomechanics literature review: Dynamics. Arboriculture \& Urban Forestry 40(1), 1-15.

James, K.R., N. Haritos, and P.K. Ades. 2006. Mechanical stability of trees under dynamic loads. American Journal of Botany 93(10):1361-1369.

Ji, J., T. Fourcaud, and Z. Zhang. 2007. Towards simulating the biomechanical acclimation of tree roots using numerical analyses. Second International Symposium on Plant Growth Modeling and Applications 78-81.

JMP®, Version 14.0 Pro. SAS Institute Inc., Cary, NC, 1989-2019.

Johnson, D. 1981. Tree hazards: recognition and reduction in recreation sites. Tech. Rep. R2-1. Lakewood, CO: USDA Forest Service.

Johnson, D., James, R. 1978. Tree hazards: recognition and reduction in recreation sites. Tech. Rep. R2-1. Lakewood, CO: USDA Forest Service.

Kaartinen, H.; Hyyppä, J.; Yu, X.; Vastaranta, M.; Hyyppä, H.; Kukko, A.; Holopainen, M.; Heipke, C.; Hirschmugl, M.; Morsdorf, F.; Næsset, E.; Pitkänen, J.; Popescu, S.; Solberg, S.; Wolf, B.M.; Wu, J.-C. An International Comparison of Individual Tree Detection and Extraction Using Airborne Laser Scanning. Remote Sens. 2012, 4, 950-974.

Kabir, E., Guikema, S., \& Kane, B. (2018). Statistical modeling of tree failures during storms. Reliability Engineering \& System Safety 177, 68-79.

Kamimura K, Gardiner B, Kato A, Hiroshima T, Shiraishi N. 2008. Developing a decisionsupport approach to reducing wind damage risk - a case study on sugi (Cryptomeria japonica (L.f.) D.Don) forests in Japan, Forestry. 
Kamimura, K., Gardiner, B., \& Koga, S. 2017. Observations and predictions of wind damage to Larix kaempferi trees following thinning at an early growth stage. Forestry 90, 530-540.

Kamimura, K., Gardiner, B., Dupont, S., Guyon, D., \& Meredieu, C. (2016). Mechanistic and statistical approaches to predicting wind damage to individual maritime pine (Pinus pinaster) trees in forests. Canadian Journal of Forest Resources 46, 88-100.

Kane, B. (2008). Tree Failure following a windstorm in Brewster, Massachusetts, USA. Urban Forestry \& Urban Greening 7, 15-23.

Kane, B. (2014). Determining parameters related to the likelihood of failure of red oak (Quercus rubra L.) from winching tests. Trees $28,1667-1677$.

Khosravipour, A.; Skidmore, A.K.; Isenburg, M.; Wang, T.; Hussin, Y.A. Generating Pit-Free Canopy Height Models from Airborne Lidar. Photogramm. Eng. Remote Sens. 2014, 80, 863-872.

Kim, H. B., \& Sohn, G. (2013). Point-based Classification of Power Line Corridor Scene Using Random Forests. Photogrammetric Engineering \& Remote Sensing, 79(9), 821-833. https://doi.org/10.14358/PERS.79.9.821

Klein, R.W., Koeser, A.K., Hauer, R.J., Hansen, G., Escobedo, F.J. 2019. Risk assessment and risk perception of trees: A review of literature relating to arboriculture and urban forestry. Arboriculture and Urban Forestry, 45(1), 23-33.

Ko, C., Remmel, T. K., \& Sohn, G. (2012). Mapping tree genera using discrete LiDAR and geometric tree metrics. Bosque (Valdivia), 33(3), 29-30. https://doi.org/10.4067/S071792002012000300015

Koeser, A. (2009). Trees \& Risk Researcher Summit White Paper.

Koeser, A.K., Smiley, E.T. 2017. Impact of assessor on tree risk assessment ratings and prescribed mitigation measures. Urban Forestry \& Urban Greening, 24, 109-115.

Koeser, R.W., Hasing, G., McLean, D., Northrop, R.J. 2013. Tree risk assessment methods: a comparison of three common evaluation forms. UF/IFAS ENH1226.

Kollman, F.; Côté, W. 1984. Principles of Wood Science and Technology; Solid Wood-Wood Based Materials; Springer: Berlin /Heidelberg, Germany, Volume I-II.

Korpela, I., Dahlin, B., Schäfer, H., Bruun, E., Haapaniemi, F., Honkasalo, J., ... Virtanen, H. (2007). Single-tree forest inventory using lidar and aerial images for 3D treetop positioning, species recognition, height and crown width estimation. International Archives of the Photogrammetry, Remote Sensing and Spatial Information Sciences, 36, 227-233. https://doi.org/10.1016/j.bmcl.2011.01.058

Korpela, I., Ole Ørka, H., Maltamo, M., Tokola, T., \& Hyyppä, J. (2010). Tree species classification using airborne LiDAR - effects of stand and tree parameters, downsizing of 
training set, intensity normalization, and sensor type. Silva Fennica, 44(2), 319-339. https://doi.org/10.14214/sf.156

Kretschmann, D.E. 2010. Mechanical properties of wood. In: Wood handbook: Wood as an engineering material. General Technical Report FPL-GTR-190. USDA, Forest Products Laboratory, Madison, Wisconsin, U.S.

Kuntz, P., Christie, R., \& Venkata, S. (2002). Optimal vegetation maintenance scheduling of overhead electric power distribution systems. IEEE Transactions on Power Delivery 17(4). 1164-1169.

Kuntz, P.A., Christie, R.D., Venkata, S.S., 2001. A reliability centered optimal visual inspection model for distribution feeders. IEEE Transactions on Power Delivery, 16(4), 718-723.

Larsen, M., Eriksson, M., Descombes, X., Perrin, G., Brandtberg, T., \& Gougeon, F. A. (2011). Comparison of six individual tree crown detection algorithms evaluated under varying forest conditions. International Journal of Remote Sensing, 32(20), 5827-5852. https://doi.org/10.1080/01431161.2010.507790

Li, W., Guo, Q., Jakubowski, M. K., \& Kelly, M. (2012). A New Method for Segmenting Individual Trees from the Lidar Point Cloud. Photogrammetric Engineering \& Remote Sensing, 78(1), 75-84. https://doi.org/10.14358/PERS.78.1.75

Lillesand, T.M., Kiefer, R.W., Chipman, J.W. 2008. Remote sensing and image interpretation. 6th ed.

Lineweber, D. and McNulty, S. (2001). The Costs of Power Disturbances to Industrial and Digital Economy Companies. Primen, Madison, Wisconsin.

Luley, C. \& Bond, J. (2006). Evaluation of the fate of ice storm-damaged urban maple (Acer) trees. Arboriculture \& Urban Forestry 32(5), 214-220.

Luley, Christopher J.; Nowak, David J.; Greenfield, Eric J. 2009. Frequency and severity of trunk decay in street tree maples in four New York cities. Arboriculture and Urban Forestry. 35(2): 94-99.

Lundström, T., M. Jonsson, A. Volkwein, and M. Stoffel. 2009. Reactions and energy absorption of trees subjected to rockfall: A detailed assessment using a new experimental method. Tree Physiology 29:345-359

Lundström, T., M. Stoffel, and V. Stöckli. 2008. Fresh-stem bending of silver fir and Norway spruce. Tree Physiology 28:355-366

Lundström, T., M.J. Jonsson, and M. Kalberer. 2007. The root-soil system of Norway spruce subject to turning moment; resistance as a function of rotation. Plant Soil 300:35-49.

Maclean, G. A., \& Krabill, W. B. (1986). Gross-merchantable timber volume estimation using an airborne lidar system. Canadian Journal of Remote Sensing, 12(1), 7-18. 
https://doi.org/10.1080/07038992.1986.10855092

Matheny, N., Clark, J. 1994. A photographic guide to the evaluation of hazard trees in urban areas. International Society of Arboriculture.

Mattheck, C., Breloer, H. 1994. Field Guide for Visual Tree Assessment (VTA). Arboriculture Journal. 18(1), 1-23.

McMahon, T.A. 1975. Using body size to understand the structural design of animals: Quadrupedal locomotion. Journal of Applied Physiology 39:619-627.

Miller KF, Quine CP, Hunt J. 1987. The assessment of wind exposure for forestry in upland Britain, Forestry, vol. 60

Miller, L.A. 2005. Structural dynamics and resonance in plants with nonlinear stiffness. Journal of Theoretical Biology 234:511-24.

Miller, R. W., Hauer, R. J., \& Werner, L. P. (2015). Urban forestry: Planning and managing urban greenspaces. Long Grove: Waveland.

Miller, R.H. 2014. Best Management Practices: Integrated Vegetation Management for Integrated Vegetation Management for Utility Rights-of-Way. International Society of Arboriculture. Champaign, IL.

Mills, L., Russell K. 1981. Detection and correction of hazard trees in Washington's recreation areas. Report 42. Olympia, WA: Washington Department of Natural Resources.

Mills, S. J., Gerardo Castro, M. P., Li, Z., Cai, J., Hayward, R., Mejias, L., \& Walker, R. A. (2010). Evaluation of aerial remote sensing techniques for vegetation management in power-line corridors. IEEE Transactions on Geoscience and Remote Sensing, 48(9), 33793390. https://doi.org/10.1109/TGRS.2010.2046905

Milne, R. 1988. The dynamics of swaying trees. New Scientist 21(1): 46.

Milne, R. 1991. Dynamics of swaying of Picea sitchensis. TreePhysiology 9(3):383-399.

Mitchell SJ, Lanquaye-Opoku N, Modzelewski H, Shen Y, Stull R, Jackson P, 2007. Comparison of wind speeds obtained using numerical weather prediction models and topographic exposure indices for predicting windthrow in mountainous terrain, For. Ecol. Manage., vol. 254 (2).

Mitchell SJ. A diagnostic framework for windthrow risk estimation, For. Chron., 1998, vol. 74.

Mohan, M., Silva, C. A., Klauberg, C., Jat, P., Catts, G., Cardil, A., ... Dia, M. (2017). Individual tree detection from unmanned aerial vehicle (UAV) derived canopy height model in an open canopy mixed conifer forest. Forests, 8(9), 1-17. https://doi.org/10.3390/f8090340 
Moore JR, Maguire DA 2004. Natural sway frequencies and damping ratios of trees: concepts, review and synthesis of previous studies. Trees 18:195-203.

Moore JR, Maguire DA. 2008. Simulating the dynamic behavior of Douglas-fir trees under applied loads by the finite element method, Tree Physiol. vol. 28.

Mortensen NG, Heathfield DN, Myllerup L, Landberg L, Rathmann O. 2005. Wind Atlas Analysis and Application Program: WAsP 8 Help Facility and On-line Documentation, Roskilde, DenmarkRis $\varnothing$ National Laboratory

Naesset, E. (2009). Determination of mean tree height of forest stands using airborne laser scanner data. ISPRS Journal of Photogrammetry and Remote Sensing, 52(2), 49-56. https://doi.org/10.1016/S0924-2716(97)83000-6

Nicoll BC, Gardiner BA, Rayner B, Peace AJ. 2006. Anchorage of coniferous trees in relation to species, soil type and rooting depth, Can. J. For. Res., vol. 36.

Nieuwenhuis, M., P.J. Fitzpatrick. 2002. An assessment of stem breakage and the reduction in timber volume and value recovery resulting from a catastrophic storm: an Irish case study. Forestry, 75, pp. 513-523.

Niklas, K. \& Spatz, H. (2000) Wind-induced stresses in cherry trees: evidence against the hypothesis of constant stress levels. Trees 14, 230-237.

Niklas, K. J. 1992. Plant biomechanics: An engineering approach to plant form and function. University of Chicago Press, Chicago, Illinois, U.S.

Niklas, K.J. 2000. Computing factors of safety against wind-induced tree damage. Journal of Experimental Botany 51:797-806.

Niklas, K.J. 2002, Wind, Size and Tree Safety. Journal of Arboriculture 28:84-93.

Paine, L. 1971. Accident hazard: evaluation and control decisions on forested recreation sites. Res. Pap. PSW-68 Berkeley, CA: USDA Forest Service.

Panshin, A.J., and C. de Zeeuw. 1980. Textbook of wood technology. McGraw-Hill, New York, New York, U.S. 722 pp.

Peltola H. 2006. Mechanical stability of trees under static loads, Am. J. Bot., vol. 93 1501-151.

Peltola, H. 1996a. Model computations on wind flow and turning moment by wind for Scots pines along the margins of clear-cut areas. Forest Ecology and Management 83(3):203215.

Peltola, H., Kellomaki, S., Vaisanen, H., \& Ikonen, V. (1999). A mechanistic model for assessing the risk of wind and snow damage to single trees and stands of Scots pine, Norway spruce, and birch. Canadian Journal of Forest Resources 29, 647-661. 
Persson, Å., \& Holmgren, J. (2004). Tree species classification of individual trees in Sweden by combining high resolution laser data with high resolution near-infrared digital images. International Archives of Photogrammetry, Remote Sensing and Spatial Information Sciences, 36(8), 204-207. Retrieved from http://www.isprs.org/proceedings/XXXVI/8W2/PERSSON.pdf

Peterson, C. \& Claassen, V. (2013). An evaluation of the stability of Quercus lobata and Populus fremontii on river levees assessed using static winching tests. Forestry 86, 201-209.

Peterson, C. (2007). Consistent influence of tree diameter and species on damage in nine eastern North America tornado blowdowns. Forest Ecology and Management 250, 96-108.

Pirotti, F. Assessing a Template Matching Approach for Tree Height and Position Extraction from Lidar-Derived Canopy Height Models of Pinus Pinaster Stands. Forests 2010, 1, 194208.

Pirotti, F.; Kobal, M.; Roussel, J.-R. A Comparison of Tree Segmentation Methods Using Very High Density Airborne Laser Scanner Data. ISPRS Int. Arch. Photogramm. Remote Sens. Spat. Inf. Sci. 2017, 42, 285-290.

Plomion, C., F. Leprovost, and A. Stokes. 2001. Wood Formation in Trees. Plant Physiology 127:1513-1523.

Pokorny, J.D. 2003. Urban tree risk management, a community guide to program design and implementation, USDA Forest Service northeastern area state and private forestry.

Popescu, S. C., \& Wynne, R. H. (2004). Seeing the trees in the forest: using lidar and multispectral data fusion with local filtering and variable window size for estimating tree height. Photogrammetric Engineering \& Remote ..., 70(5), 589-604. https://doi.org/10.14358/PERS.70.5.589

Putz, F.E., P.D. Coley, K. Lu, A. Montalvo, and A. Aiello. 1983. Uprooting and snapping of trees: Structural and ecological consequences. Canadian Journal of Forest Research 13:1011-1020.

Quine, C.P., Gardiner, B.A. 2007. Understanding how the interaction of wind and trees results in windthrow, stem breakage and canopy gap formation. - Johnson, E., Miyanishi K. (eds.). Plant Disturbance Ecology: The Process and the Response. Amsterdam, Elsevier Academic Press, 103-156.

(8) (PDF) Wind-induced stem breakage height effect on potentially recovered timber value: case study of the Scots pine (Pinus sylvestris L.) in Latvia.

R Core Team (2020). R: A language and environment for statistical computing. R Foundation for Statistical. Computing, Vienna, Austria. URL http://www.R-project.org/

Radmer, D., Kuntz, P., Christie, R., Venkata, S., \& Fletcher, R. (2002). Predicting vegetationrelated failure rates for overhead distribution feeders. IEEE Transactions on Power Delivery 17(4), 1170-1175. 
Read, J., and A. Stokes. 2006. Plant biomechanics in an ecological context. American Journal of Botany 93:1546-1565.

Reilly, A.E. 1991. The effects of Hurricane Hugo in three tropical forests in the U.S. Virgin Islands. Biotropica 23(4a): 414-419.

Rodriguez, M., E. de Langre, and B. Moulia. 2008. A scaling law for the effects of architecture and allometry on tree vibration modes suggests a biological tuning to modal compartmentalization. American Journal of Botany 95:1523-37.

Rogers, M., A. Casey, C. McMenamin. 1995. An experimental investigation of the effects of dynamic loading on coniferous trees planted on wet mineral soils. pp. 204-219 In: M.P. Coutts and J. Grace (Eds.). Wind and Trees. Cambridge University Press, Cambridge.

Roussel, JR; David Auty, Nicholas C. Coops, Piotr Tompalski, Tristan R.H. Goodbody, Andrew Sánchez Meador, Jean-François Bourdon, Florian de Boissieu, and Alexis Achim. 2020. lidR: An R package for analysis of Airborne Laser Scanning (ALS) data. Remote Sensing of Environment. 251.

Sani, L., R. Lisci, D. Sarri, M. Rimediotti, M. Vieri, and S. Tofanelli. 2012. Preliminary experiments and verification of controlled pulling tests for tree stability assessments in Mediterranean urban areas. Biosystems Engineering 112:218-226.

Schelhaas MJ, Kramera K, Peltola H, van der Werf DC, Wijdeven SMJ. 2007. Introducing tree interactions in wind damage simulation, Ecol. Model., vol. 207

Sellier D, Fourcaud T, Lac P. 2006. A finite element model for investigating effects of aerial architecture on tree oscillations, Tree Physiol., vol. 266

Sinn, G. 1990. Untersuchungen zur Kippursache von Eichen im Riederwald Frankfurt/M. Bd. LP 25. Schriftenreihe Taxationspraxis. SVK-Verlag, Erndtebrück 199S.

Shrestha, R., and Wynne R.H. (2012). Estimating Biophysical Parameters of Individual Trees in an Urban Environment Using Small Footprint Discrete-Return Imaging Lidar. Remote Sensing. 4. 484-508.

Smiley, E. T., Matheny, N. P., \& Lilly, S. (2017). Tree risk assessment. Champaign, IL: International Society of Arboriculture.

Smiley, E.T. 2008. Root pruning and stability of young willow oak. Arboriculture \& Urban Forestry 34:123-128.

Smiley, E.T., L. Holmes, and B.R. Fraedrich. 2014. Pruning of buttress roots and stability changes of red maple (Acer rubrum). Arboriculture \& Urban Forestry 40:230-236.

Smiley, E.T., N. Matheny, and S. Lilly. 2011. Best Management Practices Tree Risk Assessment. ISA, Champaign, Illinois, U.S. 
Spatz H.-C, F. Bruchert, and J. Pfisterer. 2007. Multiple resonance damping or how do trees escape dangerously large oscillations? American Journal of Botany 94:1603-11.

Spatz, H.C., and B. Theckes. 2013. Oscillation damping in trees. Plant Science 207:66-71.

Spatz, H.C., and F. Brüchert. 2000. Basic biomechanics of self supporting plants: wind and gravitational loads on a Norway spruce tree. Forest Ecology Management 135:33-44.

Spatz, H.-C.H. 2000. Greenhill's Formula for the Critical Euler Buckling Length revisited. In: H.-C.H. Spatz and T. Speck (Eds.). 3rd Plant Biomechanics.

Stokes, A. 1999. Strain distribution during anchorage failure in root systems of maritime pine (Pinus pinaster Ait.) at different ages and tree growth response to wind-induced root movement. Plant Soil 217:17-27.

Suárez, J. C., Ontiveros, C., Smith, S., \& Snape, S. (2005). Use of airborne LiDAR and aerial photography in the estimation of individual tree heights in forestry. Computers and Geosciences, 31(2), 253-262. https://doi.org/10.1016/j.cageo.2004.09.015

Suzuki, S., Sakamoto, T., \& Noguchi, H. (2016). Wind damage risk estimation for strip cutting under current and future wind conditions based on moment observations in a coastal forest in Japan. Journal for Forest Resources 21, 223-234.

Telewski, F., M.P. Coutts, J. Grace. 1995. Wind-induced physiological and developmental response in trees. Wind and Trees. Cambridge University Press, Cambridge. pp. 237-263.

Theckes, B., E. de Langre, and X. Boutillon. 2011. Damping by branching: A bioinspiration from trees. Bioinspiration and Biomimetics 6:1-11.

Tiede, D., Hochleitner, G., \& Blaschke, T. (2005). A FULL GIS-BASED WORKFLOW FOR TREE IDENTIFICATION AND TREE CROWN DELINEATION USING LASER SCANNING. In Object Extraction for 3D City Models, Road Databases, and Traffic Monitoring - Concepts, Algorithms, and Evaluation2.

Tobin, B., J. Čermák, C. Chiatante, F. Danjon, A. Di Iorio, L. Dupuy, A. Eshel,. 2007. Towards developmental modeling of tree root systems. Plant Biosystems 141:481-501.

Ussyshkin, V., \& Theriault, L. (2011). Airborne lidar: Advances in discrete return technology for 3D vegetation mapping. Remote Sensing, 3(3), 416-434. https://doi.org/10.3390/rs3030416

Vanomsen, P. 2006. Der Einfluss der Durchforstung auf die Verankerung der Fichte hinsichtlich ihrer Sturmresistenz.

Vauhkonen, J.; Ene, L.; Gupta, S.; Heinzel, J.; Holmgren, J.; Pitkänen, J.; Solberg, S.; Wang, Y.; Weinacker, H.; Hauglin, K.M.; et al. Comparative testing of single-tree detection algorithms under different types of forest. Forestry 2012, 85, 27-40. 
Virot, E., Ponomarenko, A., Dehandschoewercker, E., Quere, D., \& Clanet, C. (2016). Critical wind speed at which trees break. Physical Review E 93, 023001

Wagener, W.W. 1963. Judging hazard from native trees in California recreational areas: a guide for professional foresters. Berkeley, CA: USDA Forest Service.

Wallace, L., Lucieer, A., \& Watson, C. S. (2014). Evaluating tree detection and segmentation routines on very high resolution UAV LiDAR ata. IEEE Transactions on Geoscience and Remote Sensing. https://doi.org/10.1109/TGRS.2014.2315649

Wallace, L., Lucieer, A., Malenovskỳ, Z., Turner, D., \& Vopěnka, P. (2016). Assessment of forest structure using two UAV techniques: A comparison of airborne laser scanning and structure from motion (SfM) point clouds. Forests. https://doi.org/10.3390/f7030062

Wallace, L., Lucieer, A., Watson, C., \& Turner, D. (2012). Development of a UAV-LiDAR system with application to forest inventory. Remote Sensing.

https://doi.org/10.3390/rs4061519

Warwick, W., Hardy, T., Hoffman, M., \& Homer, J. (2016). PNNL-25178 Electricity

Distribution System Baseline Report, (July). https://doi.org/PNNL-25178

Wellpott A, Gardiner B. Salmén L. STFI-Packforsk, Stockholm. 2006. Wind loading on trees in a mature sitka spruce forest. In: Proceedings of the 5th Plant Biomechanics conference, Stockholm, Sweden, 28 August-1 September 2006. pg. 323-328.

Wessolly, L. 1991. Verfahren zur Bestimmung der Stand- und Bruchsicherheit von Bäumen [Methods for determining the safety against uprooting and stem fracture]. Holz als Rohund Werkstoff 49:99-104.

Wickham, H. (2016). ggplot2: Elegant Graphics for Data Analysis. Springer-Verlag New York. Retrieved from https://ggplot2.tidyverse.org

Woodrum, C.L., F.W. Ewers, and F.W. Telewski. 2003. Hydraulic, biomechanical, and anatomical interactions of xylem from five species of Acer (Aceraceae). American Journal of Botany 90:693-699.

Yan, W.M., L. Zhang, F.T.Y. Leung, Ka-Veng Yuen. 2016. Prediction of the root anchorage of native young plants using Bayesian inference. Urban Forestry \& Urban Greening. 19: 237252.

Zaforemska, A., W. Xiao and R. Gaulton, "Individual tree detection from UAV LiDAR data in a mixed species woodland", ISPRS Geospatial Week 2019, vol. XLII-2/W13, pp. 657-663, 2019.

Zhang, K., Chen, S. C., Whitman, D., Shyu, M. L., Yan, J., \& Zhang, C. (2003). A progressive morphological filter for removing nonground measurements from airborne LIDAR data. IEEE Transactions on Geoscience and Remote Sensing, 41(4 PART I), 872-882. 
Zobel, B.J., and J.P. van Buijtenen. 1989. Wood Variation. SpringerVerlag, Berlin, Germany. 